\title{
Investigation of Aerodynamic Braking Devices for Wind Turbine Applications
}

D. A. Griffin

$R$. Lynette \& Associates

Seattle, Washington

NREL technical monitor: Paul Migliore

\section{NPEI}

1

National Renewable Energy Laboratory

1617 Cole Boulevard

Golden, Colorado 80401-3393

A national laboratory of the U.S. Department of Energy Managed by Midwest Research Institute for the U.S. Department of Energy

under contract No. DE-AC36-83CH10093

Work performed under Subcontract No. ZAA-5-12272-05

April 1997 


\section{NOTICE}

This report was prepared as an account of work sponsored by an agency of the United States government. Neither the United States govemment nor any agency thereof, nor any of their employees, makes any warranty, express or implied, or assumes any legal liability or responsibility for the accuracy, completeness, or usefulness of any information, apparatus, product, or process disclosed, or represents that its use would not infringe privately owned rights. Reference herein to any specific commercial product, process, or service by trade name, trademark, manufacturer, or otherwise does not necessarily constitute or imply its endorsement, recommendation, or favoring by the United States govemment or any agency thereof. The views and opinions of authors expressed herein do not necessarily state or reflect those of the United States govemment or any agency thereof.

Available to DOE and DOE contractors from:

Office of Scientific and Technical Information (OSTI)

P.O. Box 62

Oak Ridge, TN 37831

Prices available by calling (423) $576-8401$

Available to the public from:

National Technical Information Service (NTIS)

U.S. Department of Commerce

5285 Port Royal Road

Springfield, VA 22161

(703) $487-4650$ 


\section{FOREWORD}

The National Wind Technology Center of the National Renewable Energy Laboratory (NREL) is supporting the efforts of its industry partners to develop advanced, utility-scale wind turbines. Part of the research being conducted focuses on innovative components and subsystems that eventually may be incorporated into these advanced turbines. R. Lynette \& Associates chose to investigate, among other technologies, the use of trailing-edge aerodynamic brakes as an alternative to the rotating tips and tip vanes that are typical of stall-controlled wind turbine rotors.

The use of trailing-edge devices for aerodynamic control of wind turbine rotors has attracted renewed interest recently, notably by New World Power Technology Company, Zond Systems, Inc., R. Lynette \& Associates and PS Enterprises, Inc.. These companies are seeking improvements in aerodynamic-braking and power-regulation systems with regard to cost, weight (or weight distribution), noise, and reliability. At first, there was considerable speculation regarding the effectiveness of various aerodynamic shapes. Most of this uncertainty has been laid to rest by the comprehensive wind-tunnel tests, and limited atmospheric tests, referenced herein. The next logical questions relate to the cost and weight of realistic implementations of trailing-edge devices. These are the issues that are addressed in this report.

As is the case with all competing technologies, the ultimate success of trailing-edge devices requires them to be embraced by a particular designer and brought to maturity by iterative cycles of design, fabrication, test, and redesign. Only then will the remaining questions of noise, icing, reliability, and maintainability be answered.

The author and his colleagues, Don Roberts, Gene Quandt, Scott Miller and Art Porter, are commended for the formulation and execution of a meticulous engineering process -- literature search, wind-tunnel tests, data analysis and system design -- all executed with precision and scrupulous attention to detail.

NREL and the U. S. Department of Energy are proud to support research activities of the high quality represented by this project and documented in this report.

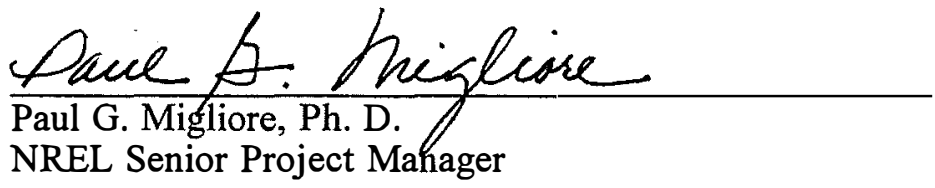




\section{PREFACE}

The present work was supported by the National Renewable Energy Laboratory (NREL) under Subcontract \#ZAA-5-12272-05, monitored by Paul Migliore. The author would like to thank Paul and others at NREL for their support on this project.

The wide range of flap configurations tested in the wind tunnel, and the quality of the data, formed a solid foundation for the sizing and deployment analyses. Art Porter of the Wichita State University (WSU) machine shop was invaluable in the detailed design and manufacture of the wind tunnel model and instrumentation. The success of the wind tunnel test was due to the outstanding work performed by Bonnie Johnson and the entire crew of the WSU wind tunnel. Scott Miller provided excellent technical support throughout the test.

Much of this project was completed by Richard Beckett, including the origination of the flip-tip concept, preliminary device selection, sizing, loads analysis, and preliminary mechanical designs. Gene Quandt also provided much support through all phases of this work. At Advanced Wind Turbines, Inc., Don Roberts performed the detailed design work, as well as the cost and weight analyses. The author would like to acknowledge and thank Richard, Gene, and Don for their invaluable contributions to this work. 


\section{ABSTRACT}

This report documents the selection and preliminary design of a new aerodynamic braking system for use on the stall-regulated AWT-26/27 wind turbines. The goal was to identify and design a configuration that offered improvements over the existing tip brake used by Advanced Wind Turbines, Inc. (AWT). Although the design objectives and approach of this report are specific to aerodynamic braking of AWT-26/27 turbines, many of the issues addressed in this work are applicable to a wider class of turbines. The performance trends and design choices presented in this report should be of general use to wind turbine designers who are considering alternative aerodynamic braking methods.

A literature search was combined with preliminary work on device sizing, loads and mechanical design. Candidate configurations were assessed on their potential for benefits in the areas of cost, weight, aerodynamic noise, reliability and performance under icing conditions. As a result, two configurations were identified for further study: the "spoiler-flap" and the "flip-tip." Wind tunnel experiments were conducted at Wichita State University to evaluate the performance of the candidate aerodynamic brakes on an airfoil section representative of the AWT-26/27 blades. The wind tunnel data were used to predict the braking effectiveness and deployment characteristics of the candidate devices for a wide range of design parameters. The evaluation was iterative, with mechanical design and structural analysis being conducted in parallel with the braking performance studies.

A spoiler-flap with active mechanical deployment was selected for detailed design. The system was designed to meet the aerodynamic braking requirements of the AWT-26 turbine. Structural analysis of the design was performed for both the device and the modified turbine blade. Although estimates showed an increased component weight of $4.9 \mathrm{~kg}(10.8 \mathrm{lb})$ per blade, this would be partially offset by improvements in weight distribution.

The preliminary estimate of the spoiler-flap system cost was $\$ 150$ less than the production AWT-26/27 tip vanes. This represents a reduction of approximately $5 \%$ in the cost of the aerodynamic braking system. In view of the preliminary nature of the design, it would be prudent to plan for contingencies in both cost and weight. Nevertheless, the results of this study are encouraging for a first-generation design. 


\section{TABLE OF CONTENTS}

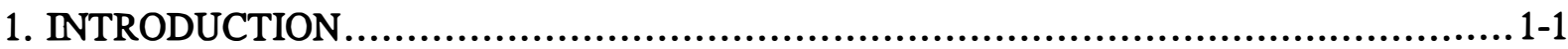

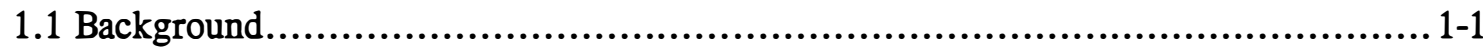

1.2 Project Schedule...........................................................................

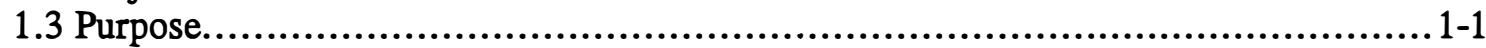

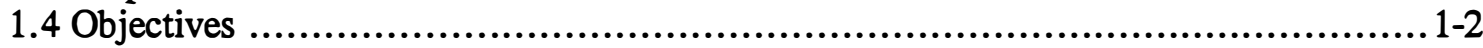

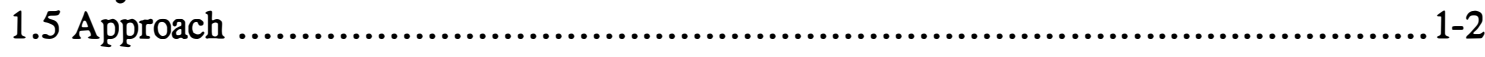

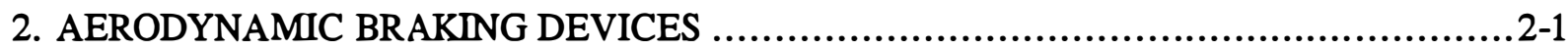

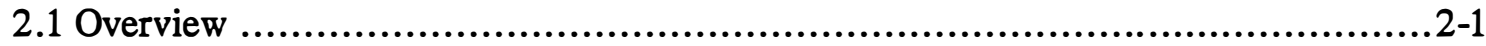

2.2 General Aerodynamic Brake Performance …...............................................2-3

3. INITIAL CONFIGURATION DEFINITIONS .................................................

3.1 Baseline Configuration, AWT-26/27 Tip Vane .............................................

3.2 Candidate Configurations....................................................................

3.2.1 Spoiler-Flap....................................................................

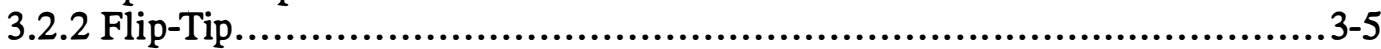

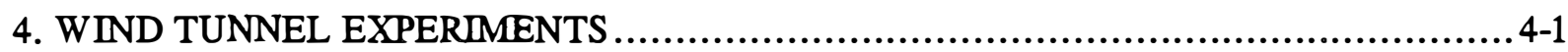

4.1 Model Design and Instrumentation ...........................................................

4.2 Test Matrix ......................................................................................

4.3 Wind Tunnel Test Results and Discussion ............................................ 4-4

4.3.1 Baseline S810 Airfoil ......................................................... 4-4

4.3.2 45\% Chord Devices................................................................ 4-4

4.3.3 Effect of Device Chord.......................................................... 4-12

4.3.4 Reynolds Number Effects ................................................... 4-13

4.3.5 Partial-Span Effects .............................................................. $4-16$

5. SIZING AND DEPLOYMENT STUDIES.....................................................

5.1 AWT-26/27 Braking Requirements .........................................................

5.2 Sizing of Candidate Devices ..............................................................

5.3 Aerodynamic Deployment Calculations.....................................................

5.3.1 Spoiler-Flap.....................................................................

5.3.2 Flip-Tip..............................................................................

5.4 Active Mechanical Deployment ..............................................................5-14

5.5 Passive Mechanical Deployment .......................................................

5.6 Configuration Selection for Detailed Design ...........................................5-18

6. DESIGN OF SPOILER-FLAP FOR AWT-26/27 ROTOR .................................... 6-1

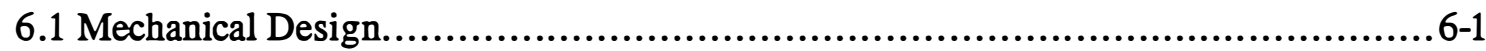

6.1 .1 Overview of Device Construction................................................6-2

6.1.2 Mechanical System Description....................................................6-3

6.2 Loads and Structural Analysis .............................................................6-6

6.2.1 Blade Loads and Structural Analysis .................................................6-6

6.2.2 Device Loads and Structural Analysis............................................6-7

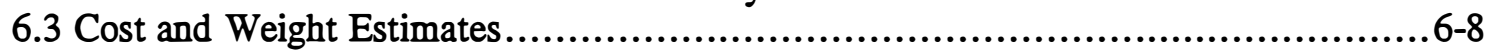

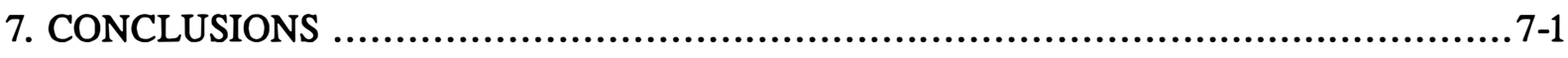

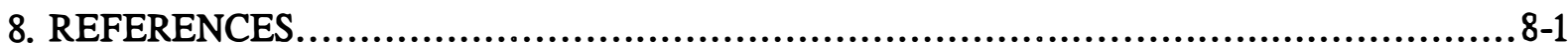




\section{LIST OF FIGURES}

Figure

Page

2-1 Velocity and Force Diagram at Radial Blade Position ..................................2-3

2-2 - Example Cp-TSR Curve for AWT-26 with Deployed Aerodynamic Brake .................2-5

3-1 General Configuration of the AWT-26/27 Turbines ...................................3-1

3-2 Major Components of AWT-26/27 Tip Vanes ............................................... $3-2$

3-3 Isometric View of Deployed Spoiler-Flap........................................... $3-4$

3-4 Isometric View of Deployed Flip-Tip.................................................

4-1 Section View of Wind Tunnel Model ....................................................4-1

4-2 End View of Flap Deflection Assembly ............................................. $4-2$

4-3 Effect of Gap Seal on 38\% Chord Device ........................................... 4-5

4-4 Effect of Gap Seal on 45\% Chord Device .......................................... 4-5

4-5 Aerodynamic Coefficients for 45\% Chord Plain Flap, Hinge A-1 ..........................4-6

4-6 Aerodynamic Coefficients for 45\% Chord Spoiler-Flap, Hinge A-3 ..................... 4-8

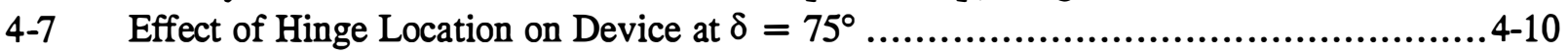

4-8 Braking Effectiveness per Unit Device Chord......................................... 4-12

4-9 Reynolds Number Effect on Spoiler-Flap .......................................... 4-14

4-10 Sectional View of Model with Partial-Span Plates .................................... 4-16

4-11 Effect of Finite Aspect Ratio on Flap Braking Effectiveness .............................. 4-17

5-1 AWT-26 Rotor Power at Varying Rotational Speeds .................................. 5-1

5-2 Rotor Power Coefficients for AWT-26 at Low TSR .......................................5-2

5-3a Sizing of Flip-Tip for AWT-26 Aerodynamic Braking .................................5

5-3b Sizing of Spoiler-Flap for AWT-26 Aerodynamic Braking ..............................5-5

5-4 Estimated Hinge Moment Data for 45\% Chord Spoiler-Flap at Hinge Location A-4.5....5-10

5-5 Rotor Power Coefficients for Deployed Spoiler-Flap ..................................5-11

5-6 Improper Spring and Damping Rates for Aerodynamically Deployed Spoiler-Flap ........5-13

5-7 Near-Optimal Spring and Damping Rates for Aerodynamic Deployment of Spoiler-Flap. 5-13

5-8 Rotor Power Coefficients for Deployed Flip-Tip ......................................5-15

5-9a Hinge Moment Data for Balanced Spoiler-Flap, Hinge A-3 .............................5-16

5-9b Hinge Moment Data for Balanced Spoiler-Flap, Hinge A-3.5 .........................5-16

5-10 Rotor Power Coefficients for Deployed Spoiler-Flap ..................................5-17

6-1 AWT-26 Blade Planform with Spoiler-Flap Location Shown ............................6-2

6-2 Spoiler-Flap Hinge / Thrust Bearing Configuration at Station 464 .........................6-3

6-3 Layout of Mechanical Actuation Driver ...............................................6-4

6-4 Sectional Layout of Mechanical Driver ............................................6 $6-5$

6-5 Station 374 Blade Section Properties ...........................................6 $6-7$ 


\section{LIST OF TABLES}

Table

Page

1-1 Major Aerodynamic Brake Development Tasks Completed .............................. 1-1

2-1 Characteristics of Current Aerodynamic Brake Designs ...................................2-2

3-1 Cost and Weight for Major Tip-Vane Subsystems ...................................... 3-2

4-1 Flap Configurations Tested by Standard $\delta$-Sweep ....................................... 4-3

5-1 Variation of Spoiler-Flap Effectiveness with Spanwise Location..........................5-6

5-2 Blade-Tip Aerodynamics for Normal Operation of AWT-26/27...........................5-10

5-3 Spring and Damping Torque Schedule for Spoiler-Flap Deployment Simulations .......... 5-12

5-4 Actuation Requirements for Active Mechanical Deployment of Spoiler-Flap..............5-18

6-1 Actuator Specifications............................................................6-5

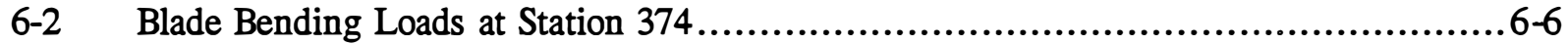

6-3 Blade Percentage Stress Increments at Station 374 .......................................6-7

6-4 Device Critical Loads ...................................................................6 6-8

6-5 Device Critical Stresses ...............................................................6 6-8

6-6 Cost and Weight Analysis Summary ................................................ 6-9 


\section{ABBREVIATIONS}

$\begin{array}{ll}\text { AWT } & \text { Advanced Wind Turbines, Inc. } \\ \text { BEMT } & \text { Blade element momentum theory } \\ \text { CER } & \text { Combined experiment rotor } \\ \text { cm } & \text { Centimeter/centimeters } \\ \mathrm{ft} & \text { Feet/foot } \\ \text { FMEA } & \text { Failure modes and effects analysis } \\ \text { ID } & \text { Inner diameter } \\ \text { in. } & \text { Inch/inches } \\ \text { kip } & \text { Kilo-pound/pounds force } \\ \text { kg } & \text { Kilogram } \\ \text { kPa } & \text { Kilopascal } \\ \text { kV } & \text { Kilovolt } \\ \text { kW } & \text { Kilowatt } \\ \text { LEGR } & \text { Leading-edge grit roughness } \\ \text { lbs } & \text { Pounds force } \\ \mathrm{m} & \text { Meter/meters } \\ \text { mm } & \text { Millimeter/millimeters } \\ \mathrm{mph} & \text { Miles per hour } \\ \mathrm{N} & \text { Newton/Newtons } \\ \text { NGIS } & \text { Next-Generation Innovative Subsystems } \\ \mathrm{NREL} & \text { National Renewable Energy Laboratory } \\ \mathrm{OD} & \text { Outer diameter } \\ \mathrm{OSU} & \text { Ohio State University } \\ \mathrm{Pa} & \text { Pascal/pascals } \\ \mathrm{psf} & \text { pounds per square foot } \\ \mathrm{psi} & \text { pounds per square inch } \\ \text { rad } & \text { Radian/radians } \\ \mathrm{RLA} & \text { R. Lynette \& Associates } \\ \mathrm{Re} & \text { Reynolds number } \\ \mathrm{rpm} & \text { Revolutions per minute } \\ \mathrm{s} & \text { Second/seconds } \\ \mathrm{WSU} & \text { Wichita State University } \\ & \end{array}$




\section{LIST OF SYMBOLS}

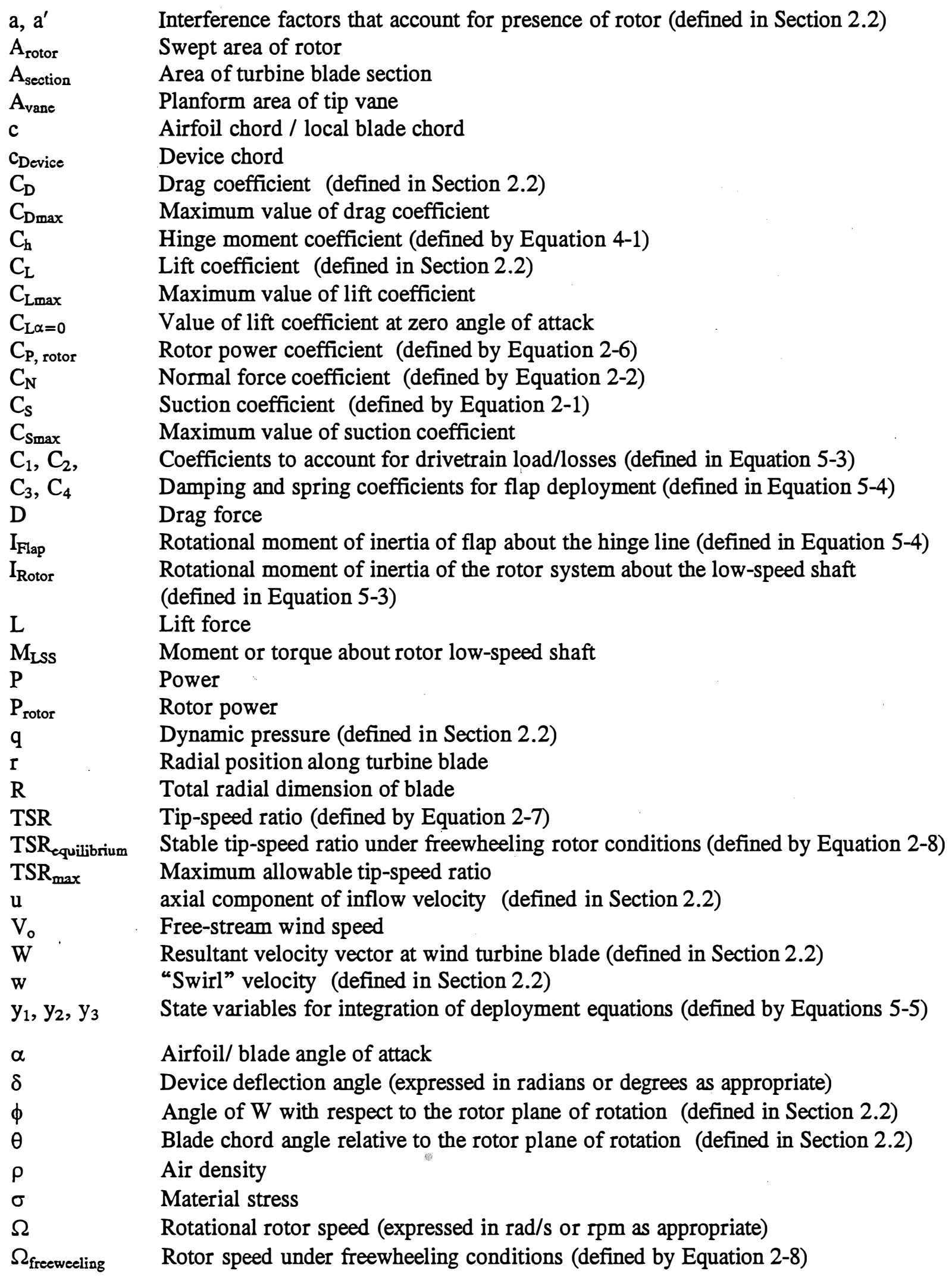




\section{Introduction}

\subsection{Background}

The R. Lynette \& Associates (RLA) Next-Generation Innovative Subsystems (NGIS) Project is designed to develop innovative subsystems which can be used to improve the performance and costeffectiveness of the AWT-26 wind turbine, and that may be usable on other advanced wind turbine designs. RLA is working cooperatively with the National Renewable Energy Laboratory (NREL) and Advanced Wind Turbines, Incorporated (AWT), on the program. The program includes the development of an improved aerodynamic brake configuration for the AWT-26/27 turbines.

\subsection{Project Schedule}

Table 1-1 summarizes the major aerodynamic braking tasks of the NGIS Project and compares the original schedule with actual completion dates. The initial tasks were completed on or ahead of schedule. Significant effort went into the selection and design of the wind-tunnel models, and the wind tunnel test was conducted 4 1/2 months later than scheduled. Due to AWT engineering priorities, no significant work was performed on aerodynamic braking between February and July of 1996. As a result, the detailed design was completed approximately 12 months later than scheduled. Prioritization of AWT resources resulted in cancellation of the planned field testing of aerodynamic brakes. This decrease in the scope of the project allowed the Draft Aerodynamic Brake Report to be completed within 6 months of the original schedule.

Table 1-1. Major Aerodynamic Brake Development Tasks Completed

\begin{tabular}{llcc}
\hline \multicolumn{1}{c}{$\begin{array}{c}\text { Innovative Subsystems } \\
\text { Task }\end{array}$} & \multicolumn{2}{c}{ Completion Dates } \\
& \multicolumn{1}{c}{ Project Schedule } & Actual \\
\hline 3.1.1 & Review Current Work & $11 / 01 / 94$ & $11 / 30 / 94$ \\
3.1.2 & Preliminary Performance and Loads Analysis & $02 / 21 / 95$ & $01 / 08 / 95$ \\
3.1.3 & Preliminary Designs & $02 / 21 / 95$ & $02 / 10 / 95$ \\
3.1 .4 & Preliminary Specification and Failure Modes & $02 / 28 / 95$ & $01 / 19 / 96$ \\
& and Effects Analysis (FMEA) & & \\
3.1 .5 & Wind Tunnel Tests & $07 / 01 / 95$ & $11 / 20 / 95$ \\
3.1 .6 & Update Cost and Performance & $07 / 14 / 95$ & $02 / 08 / 96$ \\
3.1 .7 & Final Configuration Selection & $07 / 21 / 95$ & $02 / 22 / 96$ \\
3.2 & Performance, Cost, and Structural Analysis & $08 / 01 / 95$ & $09 / 27 / 96$ \\
3.3 & Detailed Design & $09 / 14 / 95$ & $09 / 27 / 96$ \\
3.4 .1 & Draft Aerodynamic Brake Report & $04 / 12 / 96$ & $10 / 12 / 96$ \\
3.4 .2 & Final Aerodynamic Brake Report & $05 / 24 / 96$ & $02 / 17 / 96$ \\
5.5 & Aerodynamic Brake Field Test & $04 / 01 / 96$ & Canceled \\
\hline
\end{tabular}

\subsection{Purpose}

This report summarizes all significant work performed on the Aerodynamic Brake Development task of the NGIS Project. It documents the initial configuration selection, wind-tunnel testing, detailed analysis of candidate configurations, and the design and analysis of the selected aerodynamic brake configuration. 
The scope of this project did not allow for an exhaustive cost/benefit analysis of every possible design option. The design objectives and approach of this work are specific to aerodynamic braking of AWT-26/27 turbines. However, many of the issues addressed in this work may be applicable to aerodynamic braking design for a wider class of wind turbines. The performance trends and design choices presented in this report should be of general use to wind-turbine designers who are considering alternative braking methods.

\subsection{Objectives}

The objective of this project was to develop an improved aerodynamic brake configuration for use on the AWT-26/27 turbines. Goals for the new configuration included

- Decreased cost (materials and manufacturing)

- Decreased weight or better distribution of weight

- $\quad$ Reduced noise of blade tips and aerodynamic brakes

- More reliable deployment

- More robust (less maintenance)

- Ease of maintenance (accessibility of device and components)

- Device less effected by icing conditions

- Improved device scaling for future turbine modifications (rotor size and tip speed).

\subsection{Approach}

A literature search was conducted of previously reported work with aerodynamic brakes, with emphasis on applications to wind turbines. Based on initial sizing and loads analysis two candidate configurations were chosen for detailed study: the "spoiler-flap" and the "flip-tip." Two-dimensional wind tunnel tests were conducted to evaluate these devices and to develop the database necessary for performance analysis and design. Wind tunnel data were used to predict the stopping power and deployment characteristics of both the spoiler-flap and flip-tip devices. The spoiler-flap with active mechanical deployment was identified as showing the greatest promise for use on the AWT-26/27 turbines. A preliminary design and structural analysis of the selected configuration was completed. Cost and weight estimates were performed, and compared with cost and weight data for the production AWT-26/27 tip vane. 


\section{Aerodynamic Braking Devices}

\subsection{Overview}

Many wind turbine designs employ some form of aerodynamic braking to perform one or more of the following:

1. Overspeed protection - Restraint of the maximum rotor speed under a freewheeling condition (no mechanical brakes or load).

2. Power modulation - Control of power output through adjustments of aerodynamic surfaces which alter lift and drag properties over the modified blade sections.

3. Assisted mechanical braking - Aerodynamic brakes are deployed during normal braking sequences, allowing for down-sizing of mechanical brakes and decreased brake wear.

For medium-sized turbines (rated power in the $50-750 \mathrm{~kW}$ range), the most common means of overspeed control are either full-span pitch control or pitchable tips [1]. Turbines with full-span pitch control typically use this feature for both power modulation and overspeed protection. Pitchable tips may be used for power modulation, but are more frequently used only for overspeed protection and braking. For stall-regulated turbines, the most common form of overspeed protection is pitchable tips.

Tip vanes, spoiler devices, and ailerons are among the other aerodynamic brakes that have either been used successfully in the past, or are currently being used on production wind turbines. Each of these devices has its own advantages and disadvantages as summarized in Table 2-1. In the present work, alternative designs have been investigated and evaluated for improvements over currently used aerodynamic braking methods. 
Table 2-1. Characteristics of Current Aerodynamic Brake Designs

\begin{tabular}{|c|c|c|}
\hline $\begin{array}{l}\text { Aerodynamic } \\
\text { Brake }\end{array}$ & Advantages & Disadvantages \\
\hline Pitchable tips & $\begin{array}{l}\text { Combination of reduced lift and increased } \\
\text { drag occurs at blade tip, resulting in } \\
\text { optimal stopping power. } \\
\text { With active control, suitable for power } \\
\text { modulation. } \\
\text { Simple cut-line, blade/tip interface. }\end{array}$ & $\begin{array}{l}\text { Single tubular spar sees very high loads } \\
\text { during braking sequence. Mechanical } \\
\text { damping of deployment forces is difficult } \\
\text { to achieve. } \\
\text { All tip power-production loads are carried } \\
\text { through the tip/blade interface. } \\
\text { Structural/mechanical requirements lead to } \\
\text { high tip weights. }\end{array}$ \\
\hline Tip vanes & $\begin{array}{l}\text { Flat-plate drag created at maximum radial } \\
\text { position leads to high aerodynamic } \\
\text { stopping torque. } \\
\text { Centripetal/aerodynamic deployment is } \\
\text { predictable and robust, naturally has } \\
\text { greater tendency to deploy with rotor } \\
\text { overspeed. } \\
\text { Tip-plate effect gives some reduction of } \\
\text { induced drag. }\end{array}$ & $\begin{array}{l}\text { Creates high braking torque, but must } \\
\text { overcome all power-production torque of } \\
\text { the (unmodified) clean blade. } \\
\text { No relief of clean-blade lift results in } \\
\text { higher flapwise blade loads. } \\
\text { Wetted area of tip vane, along with the } \\
\text { corner-flow in the tip/blade junction, can } \\
\text { cause increases in both drag and noise } \\
\text { during normal turbine operation. } \\
\text { Not suitable for power modulation. }\end{array}$ \\
\hline Spoilers & $\begin{array}{l}\text { Creates effective braking by reducing lift } \\
\text { and creating drag over the modified } \\
\text { portion of the blade. } \\
\text { Active control can be used to restrict peak } \\
\text { power, but only by "power shaving." } \\
\text { Actuation loads low for power shaving. }\end{array}$ & $\begin{array}{l}\text { Stopping power diminishes in airfoil post- } \\
\text { stall as spoiler is engulfed by the wake. } \\
\text { Deployment loads high during emergency } \\
\text { braking. }\end{array}$ \\
\hline Ailerons & $\begin{array}{l}\text { With active control, can be used to } \\
\text { modify lift and drag of blade section, for } \\
\text { either power modulation or for braking. } \\
\text { Trailing-edge devices can have small } \\
\text { effect on aerodynamic drag when stowed. } \\
\text { Potential for active teeter control. }\end{array}$ & $\begin{array}{l}\text { Stopping power diminishes in airfoil post- } \\
\text { stall as aileron is engulfed by the wake } \\
\text { and overall projected area decreases. } \\
\text { Deployment loads high during emergency } \\
\text { braking. }\end{array}$ \\
\hline
\end{tabular}




\subsection{General Aerodynamic Brake Performance}

The following aerodynamic analysis uses the blade element momentum theory (BEMT), which relies on the assumption that radial blade sections can be analyzed independently [2]. For blade sections in the vicinity of an aerodynamic braking device, this assumption may be poor. The validity of using BEMT methods to analyze aerodynamic brakes will be addressed further in following sections. Figure 2-1 shows a diagram of a radial blade element, where:

L $\quad \equiv$ lift force, acting perpendicular to the resultant velocity vector

$\mathrm{D} \equiv \mathrm{drag}$ force, acting parallel to the resultant velocity vector

$\mathrm{W} \equiv$ resultant velocity vector at blade section, $\left(\mathrm{u}^{2}+(\mathrm{r} \cdot \Omega+\mathrm{w})^{2}\right)^{1 / 2}$

$\mathrm{V}_{0} \quad \equiv$ free-stream wind velocity

$\mathrm{u} \equiv$ axial component of inflow velocity

$\mathrm{w} \quad \equiv$ "swirl" velocity

$\mathrm{r} \cdot \Omega \equiv$ velocity caused by blade rotation

$\alpha \quad \equiv$ blade angle of attack relative to the resultant velocity vector, $\mathrm{W}$

$\theta \quad \equiv$ blade chord relative to the rotor plane of rotation

$\phi \quad \equiv$ angle of the resultant velocity with respect to the rotor plane of rotation

$\mathrm{a}, \mathrm{a}^{\prime} \equiv$ interference factors which account for presence of rotor.

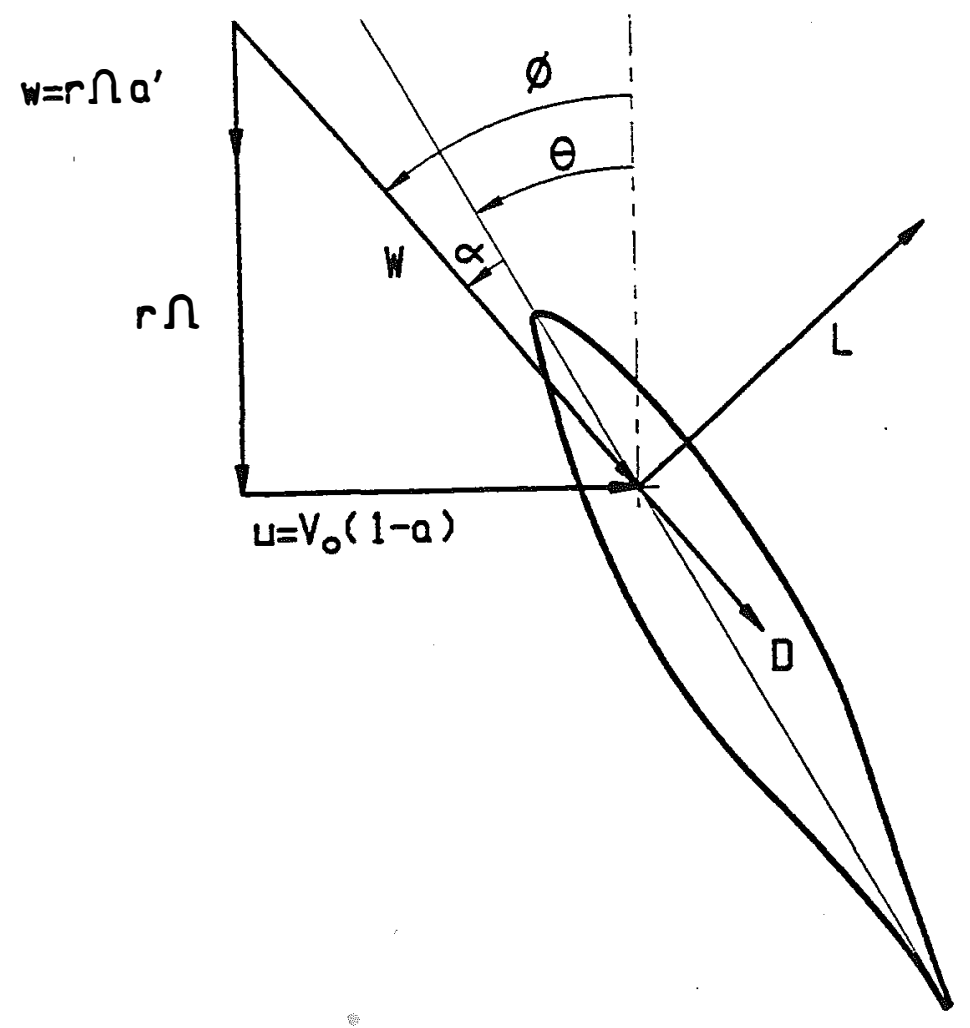

Figure 2-1. Velocity and Force Diagram at Radial Blade Position 
The local lift and drag can be expressed in coefficient form as:

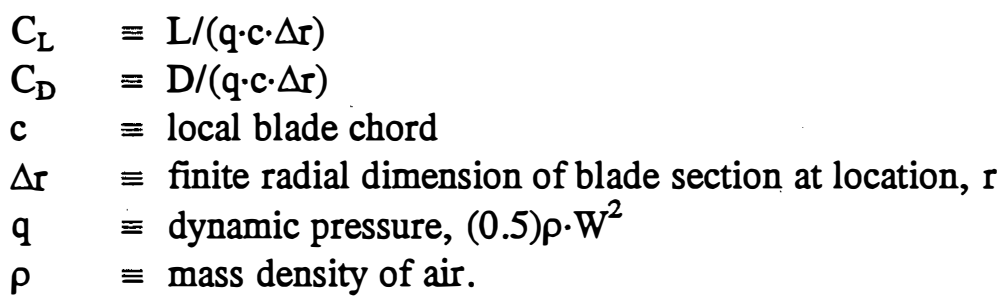

Resolving force vectors into the rotor plane of rotation, a "suction" coefficient can be defined that is related to the production of useful torque:

$C_{S}=C_{L} \cdot \sin (\phi)-C_{D} \cdot \cos (\phi)$

Similarly, a "normal force" coefficient can be defined which is related to blade thrust loading:

$C_{N}=C_{L} \cdot \cos (\phi)+C_{D} \cdot \sin (\phi)$.

Based on the above definitions, expressions can be obtained for the incremental torque (Q), thrust (T), and power $(\mathrm{P})$ generated by a single blade section located at radius $\mathrm{r}$ :

$\Delta \mathrm{Q}=\mathrm{q} \cdot \mathrm{r} \cdot \mathrm{C}_{\mathrm{s}} \cdot \mathrm{c} \cdot \Delta \mathrm{r}$

$\Delta \mathrm{T}=\mathrm{q} \cdot \mathrm{C}_{\mathrm{N}} \cdot \mathrm{c} \cdot \Delta \mathrm{r}$

$\Delta \mathrm{P}=\Omega \cdot \Delta \mathrm{Q}=\Omega\left[\mathrm{q} \cdot \mathrm{r} \cdot \mathrm{C}_{\mathrm{S}} \cdot \mathrm{c} \cdot \Delta \mathrm{r}\right]$

The above definitions and equations contain the essentials of normal turbine power production, as well as aerodynamic braking. Within the assumptions of BEMT, an iterative solution is available for the interference factors $a$ and $a^{\prime}$. For a given wind speed, $V_{o}$, the blade angle of attack, $\alpha$, can be related to $\phi$ through the rotor geometry, blade pitch setting, and twist.

Assuming that no mechanical torque is applied to the rotor shaft, an equilibrium freewheeling speed is attained when the negative torque produced by the blade sections with deployed aerodynamic brakes is exactly balanced by the positive torque contributions from the remaining blade. Inspection of Equation 2-1 shows that at low blade angles of attack, generating negative values of $C_{S}$ is achieved more directly by increasing drag than by decreasing lift.

Rotor performance is often expressed in terms of power coefficient $\left(\mathrm{C}_{\mathrm{P}}\right)$ versus tip-speed ratio (TSR):

$$
\begin{aligned}
& \mathrm{C}_{\mathrm{P}, \text { rotor }} \equiv \frac{\mathrm{P}_{\text {rotor }}}{0.5 \cdot \rho \cdot \mathrm{V}_{\mathrm{o}}^{3} \cdot \mathrm{A}_{\text {rotor }}} \\
& \mathrm{TSR} \equiv \frac{\text { Rotor Tip Speed }}{\text { Wind Speed }}=\frac{\mathrm{R} \cdot \Omega}{\mathrm{V}_{\mathrm{o}}}
\end{aligned}
$$


where:

$A_{\text {rotor }} \equiv$ swept area of rotor $\left(\pi \cdot R^{2}\right.$ for a horizontal-axis wind turbine)

$\mathrm{R} \quad \equiv$ radius of turbine rotor

$\Omega \quad \equiv$ rotational speed of rotor $(\mathrm{rad} / \mathrm{s})$.

Note that the rotor power coefficient is normalized with respect to the free-stream wind speed. By contrast, the expressions for torque, thrust, and power are all in terms of the resultant velocity vector which has a large rotational component. TSR is a non-dimensional tip speed, and the geometric angle of attack for each blade section is equal to $\tan ^{-1}\left(V_{0} / r \cdot \Omega\right)$.

Figure 2-2 shows $C_{\mathrm{p}}$-TSR curves for the AWT-26 turbine, including the effect of an aerodynamic brake at several deployment angles. Such a plot can easily be used to determine the equilibrium tipspeed ratio for a turbine in the freewheeling condition. Using Figure 2-2 as an example, the $\mathrm{C}_{\mathrm{p}}$ curve for $\delta=30^{\circ}$ has its highest zero crossing at a tip-speed ratio of about 7.5. For tip speeds lower than this value, the rotor will create positive mechanical power and accelerate. Conversely, the rotor will decelerate for tip-speed ratios above this zero crossing. For a deployed aerodynamic brake, the equilibrium tip-speed ratio will determine the maximum freewheeling rotor speed as a function of wind speed. Rearranging Equation 2-7, and evaluating for the equilibrium condition, yields:

$$
\Omega_{\text {freewheeling }}=\frac{\mathrm{TSR}_{\text {equilibrium }} \cdot \mathrm{V}_{\mathrm{o}}}{\mathrm{R}} \text {. }
$$

This report will make frequent use of Cp-TSR curves for device sizing and for evaluating aerodynamic brake performance, as the curves may be used to represent a wide range of wind conditions and turbine rotational speeds.

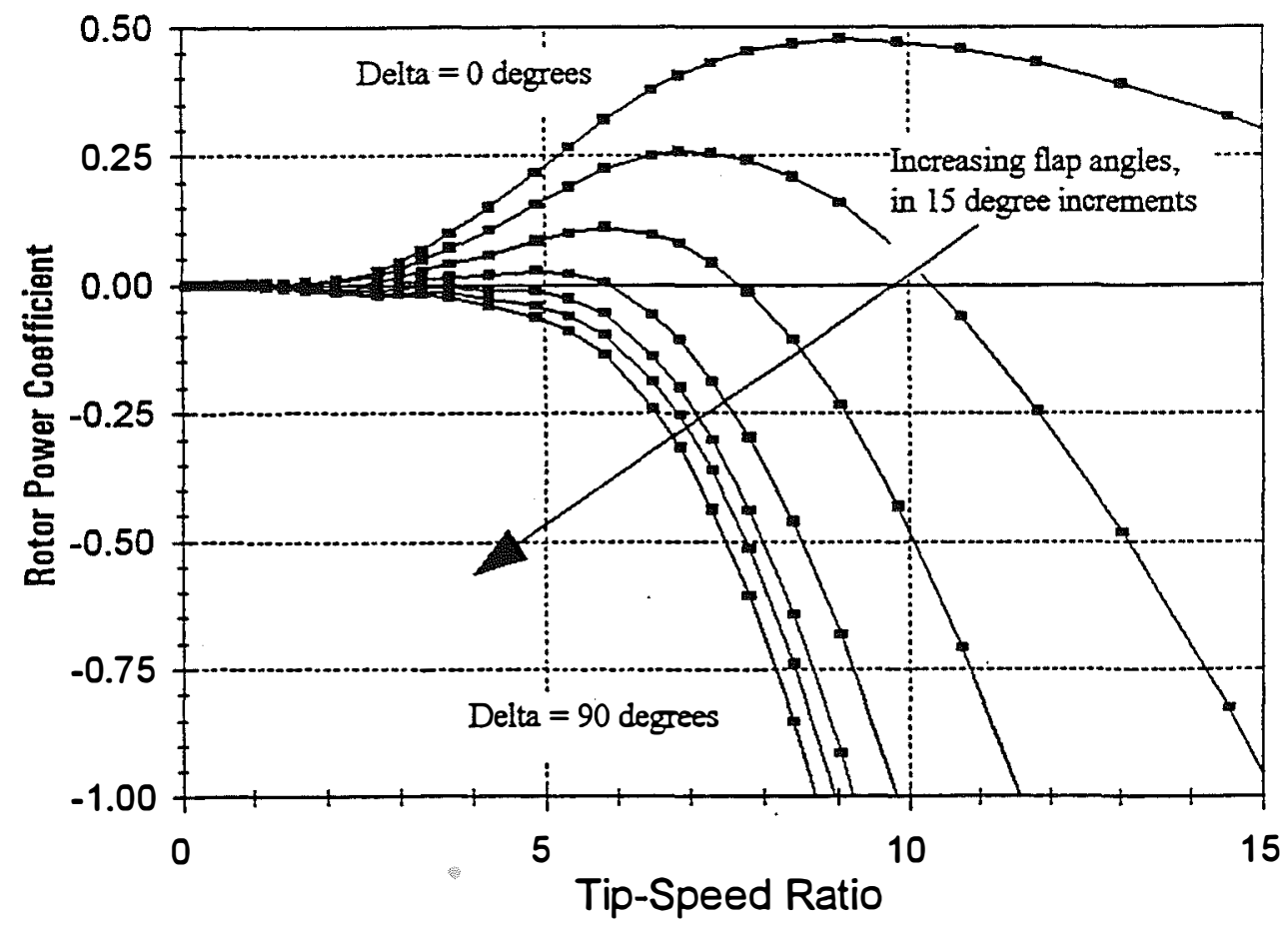

Figure 2-2. Example Cp-TSR Curve for AWT-26 with Deployed Aerodynamic Brake 


\section{Initial Configuration Definitions}

\subsection{Baseline Configuration, AWT-26/27 Tip Vane}

The baseline configuration for this work is the AWT 26/27 tip vane, which has been used successfully on several AWT-26 prototypes, and on production AWT-27 turbines. Figure 3-1 shows the general configuration of the AWT-26/27, which is a downwind, free-yaw turbine. The rotor is a two-bladed, fixed-pitch, stall-regulated design, which achieves high efficiency through the use of NREL

S815/S809/S810 airfoils. The blades are made of wood-epoxy laminates, reinforced with carbon fiber. The AWT-26 has a diameter of $26.2 \mathrm{~m}(86 \mathrm{ft})$ and a nominal rotational speed of $57 \mathrm{rpm}$. The AWT27 has a $27.4 \mathrm{~m}(90 \mathrm{ft})$ diameter with a nominal rotational speed of $53 \mathrm{rpm}$.

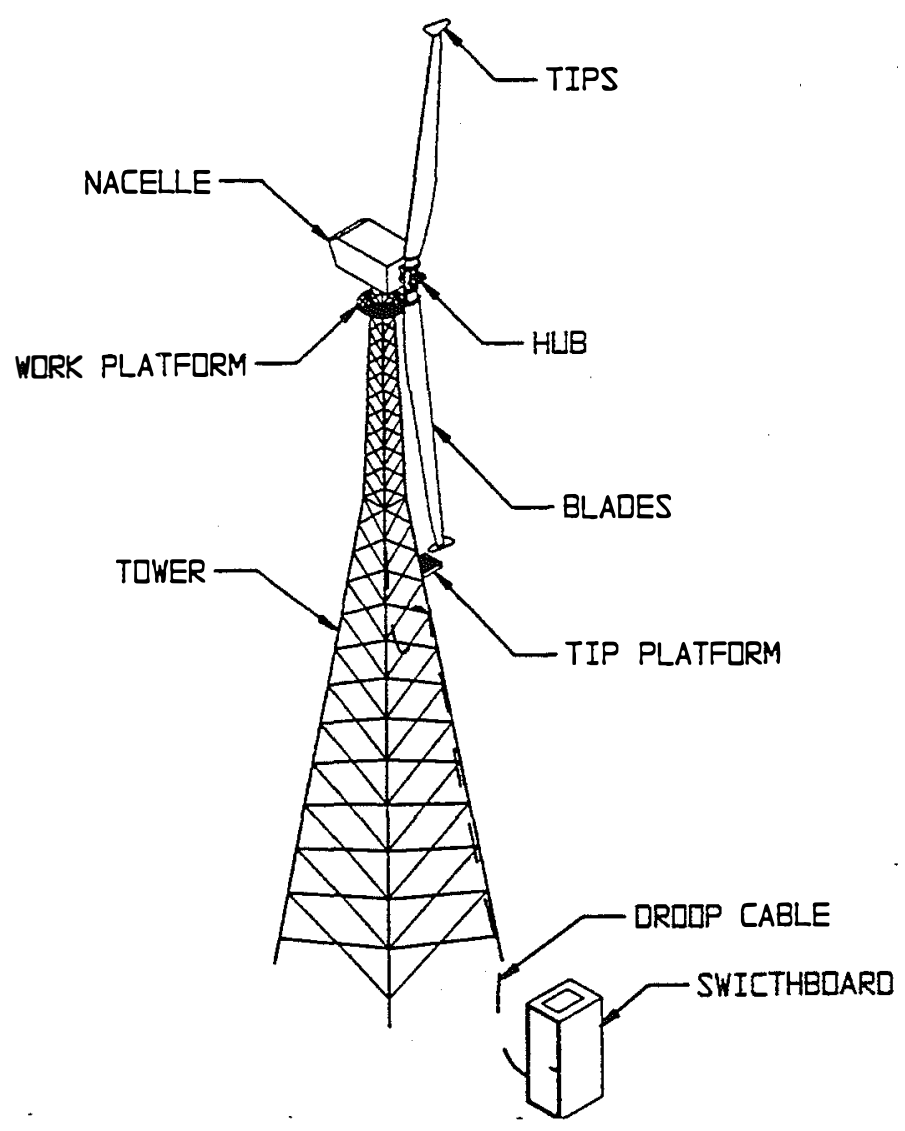

Figure 3-1 General Configuration of AWT-26/27 Turbines

Figure 3-2 identifies the major tip-vane components and illustrates the vane orientation in both stowed and deployed positions. The tip brakes provide assistance to the mechanical brakes during all rotor stopping sequences, as well as being a redurdant means of preventing rotor overspeed for all wind speeds. The tip brakes consist of a vane and an aerodynamic fairing that covers the deployment hardware (striker plate, hinge, baseplate, damper, spring, electrical magnet, and tip studs). The vane is a composite construction of structural foam overlaid with carbon fiber fabric, epoxy, and gelcoat. The hinge assembly allows the brake to move from the stowed (turbine operating) to the braking position. 
During normal turbine operation, the magnet and springs hold the vane perpendicular to the blade axis so that the vane produces minimal resistance as the rotor turns. A tip brake deploys when electric current is cut off from the electrical magnet while the rotor is turning. Centrifugal forces cause the trailing edge of the vane to move outw ard, exposing the relatively flat surface of the vane to the incident wind direction. Drag generated by the tip vane opposes the torque applied to the rotor by aerodynamic lift on the blades. As the rotor slows, the vane is returned to its operating position by the balance of centrifugal, aerodynamic, and spring forces. The return motion is moderated by a viscous damper.

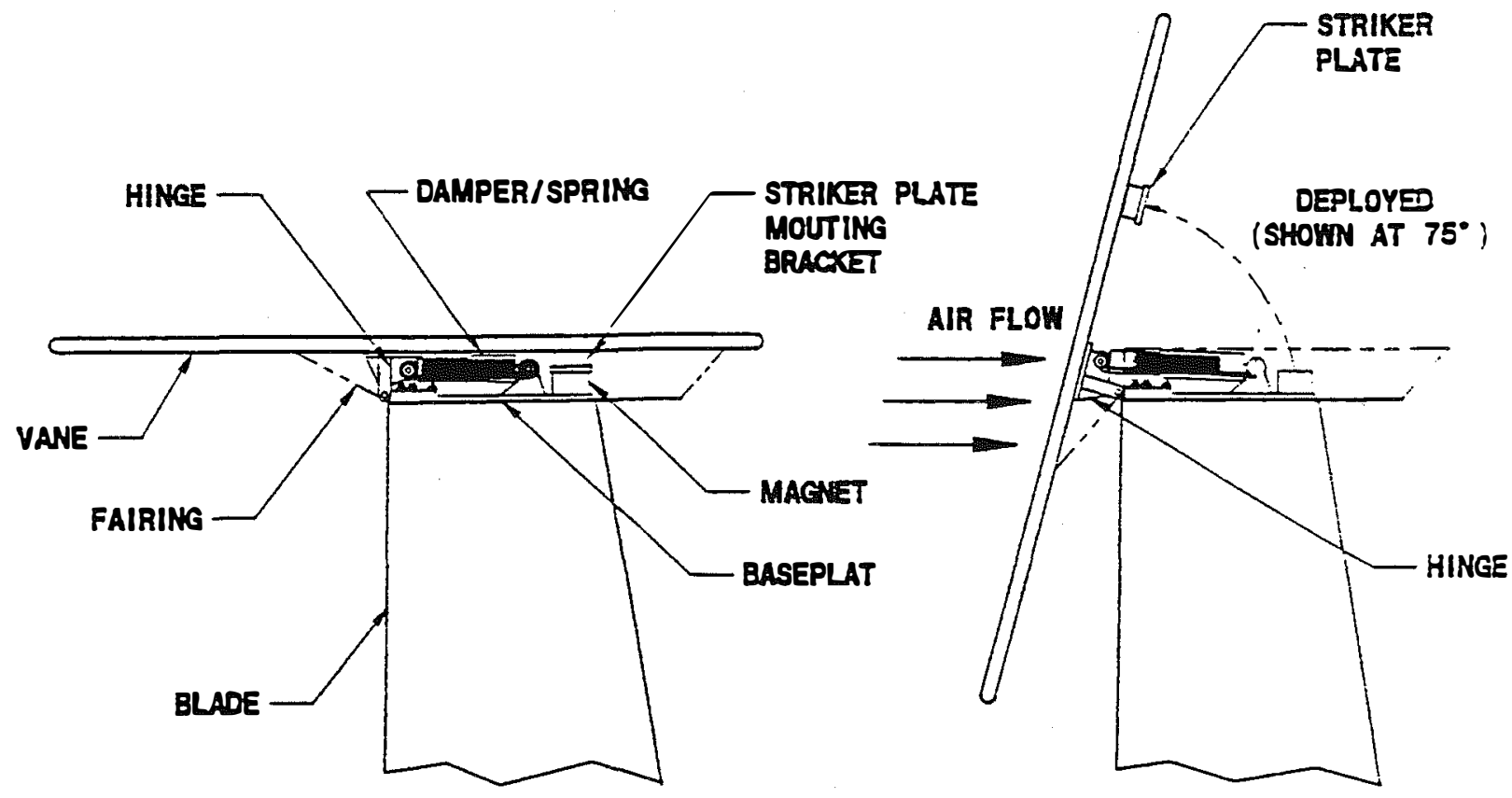

Figure 3-2. Major Components of AWT-26/27 Tip Vanes

A slip-ring assembly is used to carry 120 -volt AC power across the rotating interface between the nacelle and the hub. An AC to DC rectifier is located in a small junction box on the hub. The rectifier converts the $\mathrm{AC}$ electricity coming from the turbine controller to DC electricity used to energize the electromagnets at the blade tips. The tip vanes are fail safe, and will deploy any time there is a loss of DC power to the electromagnets (loss of grid power, failed connection, etc.).

Table 3-1 shows production costs and weights for the major subsystems of the AWT-26/27 tip vanes. The entry titled "mounting hardware" includes the mounting plate, hinge assembly, spring, damper, electromagnet, and all miscellaneous studs, pins, bushings, and other hardware.

Table 3-1. Cost and Weight for Major Tip-Vane Subsystems

\begin{tabular}{cccrr}
\hline $\begin{array}{c}\text { Major Components / } \\
\text { Subsystem }\end{array}$ & $\begin{array}{c}\text { \# Per } \\
\text { Turbine }\end{array}$ & Cost $(\$)$ & Weight & Cost/Weight \\
\hline Vane and Fairing & 2 & 833.34 & $6.3 \mathrm{~kg}(13.9 \mathrm{lbs})$ & $132 \$ / \mathrm{kg}(60.0 \$ / \mathrm{lb})$ \\
Mounting Hardware & 2 & 465.35 & $6.4 \mathrm{~kg}(14.0 \mathrm{lbs})$ & $16.5 \$ / \mathrm{kg}(33.3 \$ / \mathrm{lb})$ \\
Slip Ring and Rectifier & 1 & $1,712.00$ & $14.4 \mathrm{~kg}(31.8 \mathrm{lbs})$ & $26.7 \$ / \mathrm{kg}(53.8 \$ / \mathrm{lb})$ \\
\hline
\end{tabular}


The aerodynamic performance of the tip vane is relatively simple. Ignoring tip-plate effects, deployment of the tip brakes leaves the turbine rotor aerodynamically unchanged. To a good approximation, the negative (stopping) power provided by the tip vanes can be added linearly to the positive power produced by the turbine blade. The tip vane remains nearly neutral with respect to the free-stream wind vector both while stowed and while deployed. The apparent velocity of the tip brake can then be completely characterized by the rotational velocity of the blade tip, $R \cdot \Omega$, and the stopping power related to the device drag by the following equations (for one vane):

$$
\begin{aligned}
& \mathrm{D}=\mathrm{q} \cdot \mathrm{C}_{\mathrm{D}} \cdot \mathrm{A}_{\text {vane }}=0.5 \cdot \rho \cdot \mathrm{V}^{2} \cdot \mathrm{C}_{\mathrm{D}} \cdot \mathrm{A}_{\text {vane }} \\
& \Delta \mathrm{P}=-\mathrm{D} \cdot \Omega \cdot \mathrm{R}=-0.5 \cdot \rho \cdot \mathrm{C}_{\mathrm{D}} \cdot \mathrm{A}_{\text {vane }} \cdot(\Omega \cdot \mathrm{R})^{3}
\end{aligned}
$$

where the substitution of $V^{2}=(R \cdot \Omega)^{2}$ has been used. Multiplying the right-hand side by $\left(V_{0} / V_{0}\right)^{3}$ yields (for one vane):

$$
\Delta \mathrm{P}=-0.5 \cdot \rho \cdot \mathrm{C}_{\mathrm{D}} \cdot \mathrm{A}_{\text {vane }} \mathrm{TSR}^{3} \cdot \mathrm{V}_{\mathrm{o}}^{3}
$$

Equations 3-3 and 2-6 can be combined to express aerodynamic braking power in terms of rotor power coefficient (for two tip vanes):

$$
\Delta \mathrm{C}_{\mathrm{P} \text {, rotor }}=-2 \cdot \mathrm{C}_{\mathrm{D}} \cdot \mathrm{TSR}^{3} \frac{\mathrm{A}_{\text {vane }}}{\mathrm{A}_{\text {rotor }}}
$$

Equation 3-4 provides a simple expression for the aerodynamic effectiveness of tip vanes, and clearly

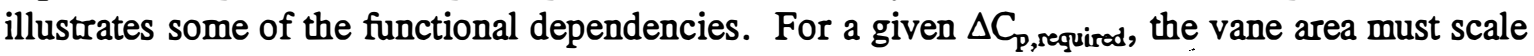
linearly with the rotor swept area, and the device effectiveness increases rapidly with tip-speed ratio. In terms of rotor scaling, Equation 3-4 must be interpreted with care. Note that $\mathrm{C}_{\mathrm{p}}$ is normalized by rotor area, so at fixed values of rotor power and TSR, the $\Delta \mathrm{C}_{\mathrm{p} \text {, required }}$ for effective braking would decrease as $1 / A_{\text {rotor }}$ for larger rotor diameters.

\subsection{Candidate Configurations}

To identify promising configurations for aerodynamic braking devices, a review of existing literature was conducted. Data obtained through the literature review, including recent wind tunnel testing of trailing-edge aerodynamic brakes [ 3 and 4], were used as a basis for a preliminary sizing and loads analysis. Based on the analysis, two candidate configurations were selected for further investigation, the "spoiler-flap" and the "flip-tip." These configurations were identified as showing the greatest promise for incorporating into the AWT-26/27 design, and for meeting the objectives as listed in Section 1.4.

\subsubsection{Spoiler-Flap}

The spoiler-flap was jointly conceived by Gene Quandt and NREL, and is shown schematically in Figure 3-3. The trailing edge of the spoiler-flap is rotated downward during deployment. The forward portion of the device disrupts the flow on the low-pressure side of the airfoil as it protrudes upward. The effectiveness of the spoiler-flap is based on a moderate amount of drag creation, coupled 
with large reductions in torque-producing lift. An important feature is the venting of air flow from the high-pressure to low-pressure side of the airfoil, which tends to prevent the flap from acting as a highlift device.

The hinge moments of spoiler-flaps can be strongly affected by the relative position of the hinge line. Spoiler-flaps are generally suitable for deployment by either aerodynamic, active mechanical, or passive mechanical methods, any of which may make use of the centripetal accelerations acting on the device or other mechanisms. Deployment methods are addressed in greater detail in Section 5.0.

In addition to the work of References 3 and 4, wind-tunnel tests of spoiler-flaps and ailerons have been conducted by Ohio State University (OSU) [5], and atmospheric testing has been conducted using the NREL Combined Experiment Rotor (CER) [6]. The publication of these recent tests results is pending. Quandt is currently working on the testing of prototype spoiler-flaps [7], and has published the preliminary design of spoiler-flaps for a Micon 65/13 [8].

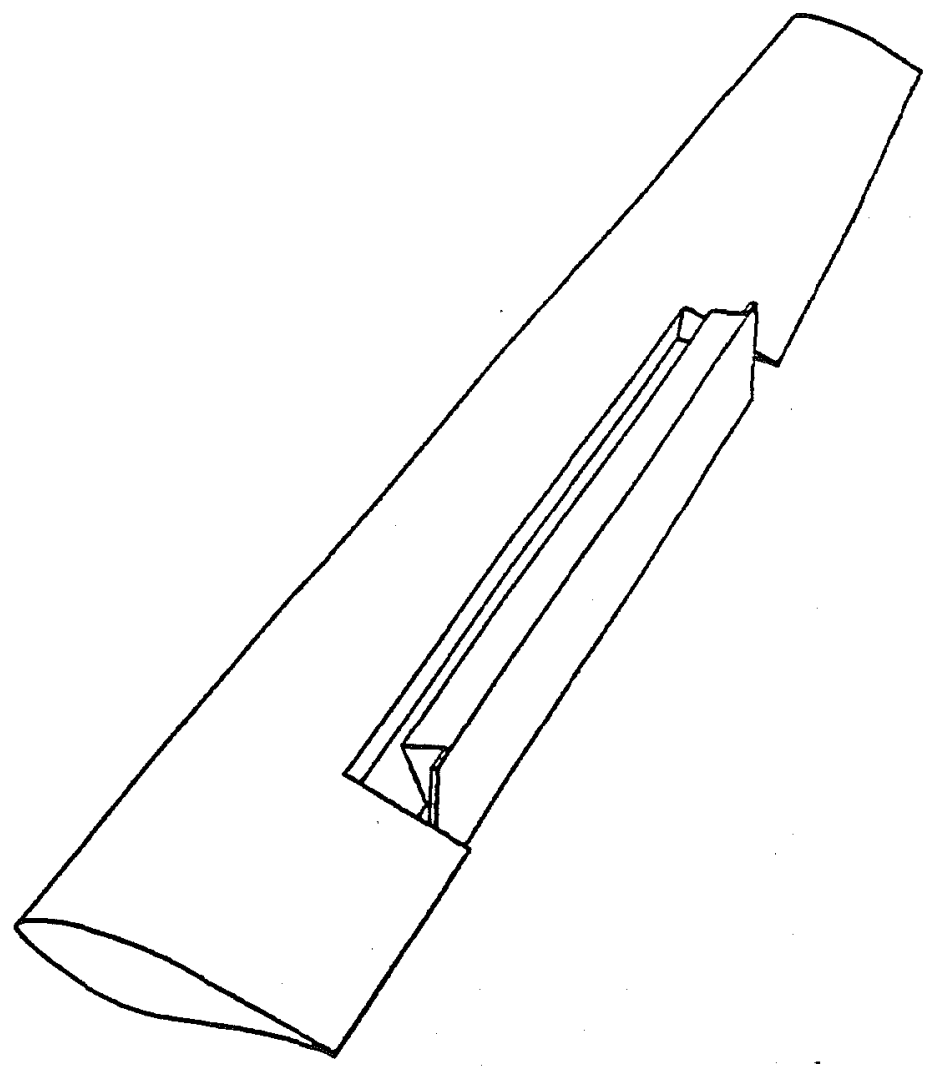

Figure 3-3. Isometric View of Deployed Spoiler-Flap

Beginning with the force and velocity vectors defined in Figure 2-1, and making use of Equations 2-1 through 2-7, expressions can be derived for the effects of a device which modifies the local blade aerodynamic properties (such as a spoiler-flap). From Figure 2-1:

$$
\mathrm{W}=\frac{\mathrm{r} \cdot \Omega\left(1+\mathrm{a}^{\prime}\right)}{\cos (\phi)}
$$


Near the tip regions of a wind turbine blade, the radial interference factor, $a^{\prime}$, is generally small. Therefore, a good approximation to the resultant velocity vector, $\mathrm{W}$, is given by:

$$
\mathrm{W} \approx \frac{\mathrm{r} \cdot \Omega}{\cos (\phi)}
$$

Using Equation 3-6 in a derivation similar to that for the tip vane leads to expressions for the incremental changes in rotor power and rotor power coefficient (for both blades modified):

$$
\begin{aligned}
& \Delta \mathrm{P}=\rho \cdot \frac{\left\{\Delta \mathrm{C}_{\mathrm{L}} \sin (\phi)-\Delta \mathrm{C}_{\mathrm{D}} \cos (\phi)\right\}}{\cos ^{2}(\phi)} \cdot \mathrm{A}_{\text {section }} \cdot\left(\frac{\mathrm{r}}{\mathrm{R}}\right)^{3} \cdot \mathrm{TSR}^{3} \cdot \mathrm{V}_{\mathrm{o}}^{3} \\
& \Delta \mathrm{C}_{\mathrm{P}, \text { rotor }}=2 \cdot\left(\frac{\mathrm{r}}{\mathrm{R}}\right)^{3} \cdot \mathrm{TSR}^{3} \frac{\left\{\Delta \mathrm{C}_{\mathrm{L}} \sin (\phi)-\Delta \mathrm{C}_{\mathrm{D}} \cos (\phi)\right\}}{\cos ^{2}(\phi)} \cdot \frac{\mathrm{A}_{\text {section }}}{\mathrm{A}_{\text {rotor }}} \\
& =2 \cdot\left(\frac{\mathrm{r}}{\mathrm{R}}\right)^{3} \cdot \mathrm{TSR}^{3} \frac{\Delta \mathrm{C}_{\mathrm{S}}}{\cos ^{2}(\phi)} \cdot \frac{\mathrm{A}_{\text {section }}}{\mathrm{A}_{\text {rotor }}} \text {. }
\end{aligned}
$$

Where $\Delta C_{L}, \Delta C_{D}$, and $\Delta C_{S}$ are the incremental changes in lift, drag, and suction coefficients of the local blade sections due to the deflection of the spoiler-flap device, and $\mathrm{r}$ is a radial position which characterizes the device. In terms of functional dependencies, Equations 3-8 and 3-4 are very similar. The device effectiveness scales linearly with the area of the modified blade section, and as a cubic of both the tip-speed ratio and the radial position.

The spoiler-flap has several favorable attributes for wind turbine aerodynamic braking. Wind tunnel data show that spoiler-flaps can maintain high negative values of $\mathrm{C}_{S}$ over a wide range of airfoil angles of attack. The device aerodynamic surfaces and supports are aft on the blade, reducing the drag penalty due to the device gap as well as minimizing the loads carried during normal power production. Spoiler-flaps may also be used for power modulation, as they can produce significant negative $\Delta \mathrm{C}_{\mathrm{s}}$ at low deflection angles.

\subsubsection{Flip-Tip}

The flip-tip, which was conceived at AWT and is a hybrid of a pitchable tip and a plain flap, is shown in Figure 3-4. The flip-tip was designed to be aerodynamically deployed (trailing-edge down). With proper sizing and hinge location, the tip region provides positive opening moments for all blade angles of attack. The tip region provides maximum braking torque as it is deployed towards stall at higher angles of attack. The flap region is intended to provide aerodynamic damping so that the flip-tip assembly reaches an equilibrium deployment angle prior to experiencing a hard mechanical stop. The aerodynamic stopping loads of the tip are transferred to the main blade structure through the flap and hinges. 


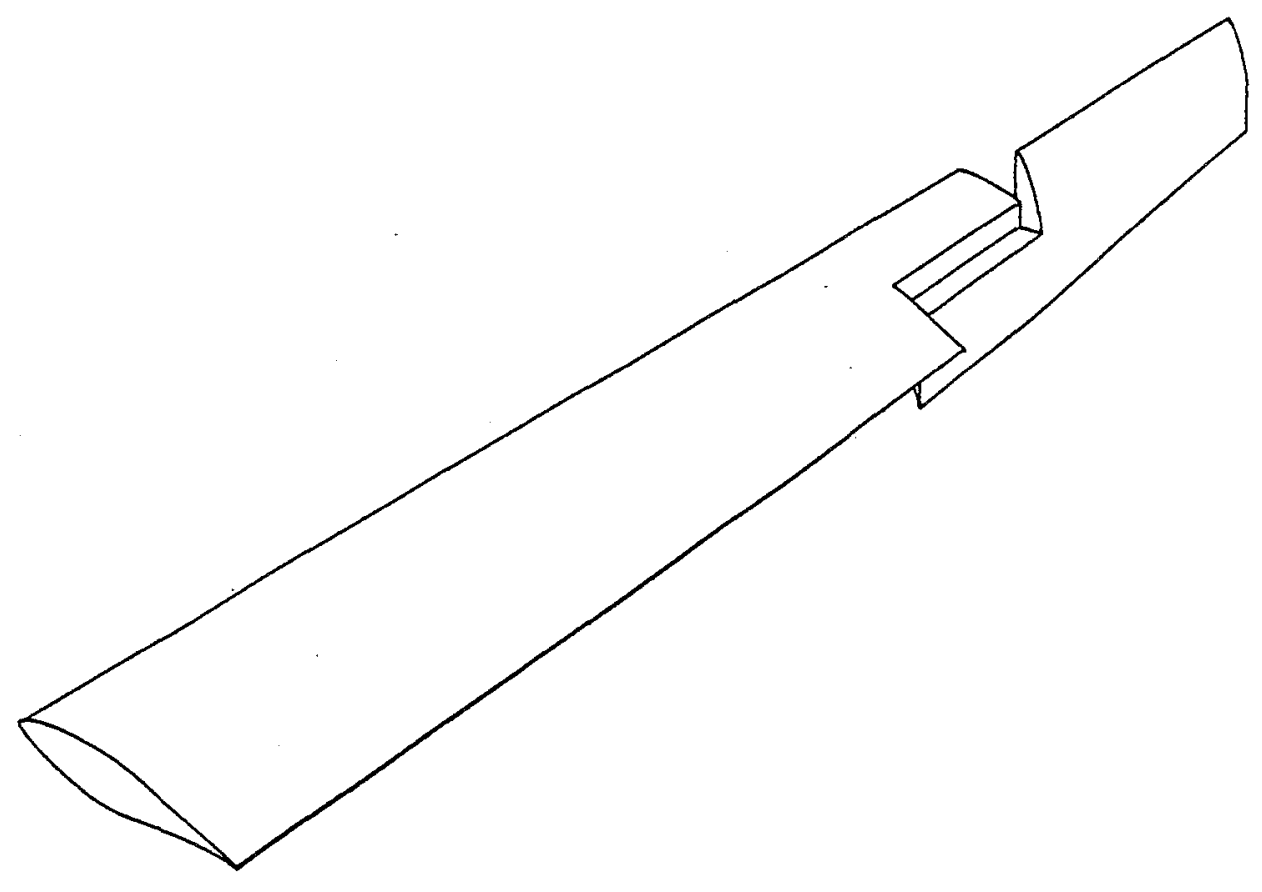

Figure 3-4. Isometric View of Deployed Flip-Tip

The aerodynamic performance of the flip-tip may be approximated by a linear superposition of the tip and flap regions. The resulting expression for the change in rotor power coefficient shows the same functional dependencies as noted for the spoiler-flap:

$$
\Delta \mathrm{C}_{\mathrm{P}, \text { rotor }}=\frac{2 \cdot \mathrm{TSR}^{3}}{\mathrm{~A}_{\text {rotor }}}\left[\left\{\left(\frac{\mathrm{r}}{\mathrm{R}}\right)^{3} \cdot \frac{\Delta \mathrm{C}_{\mathrm{S}}}{\cos ^{2}(\phi)} \cdot \mathrm{A}_{\text {section }}\right\}_{\mathrm{flap}}+\left\{\left(\frac{\mathrm{r}}{\mathrm{R}}\right)^{3} \cdot \frac{\Delta \mathrm{C}_{\mathrm{S}}}{\cos ^{2}(\phi)} \cdot \mathrm{A}_{\text {section }}\right\}_{\text {tip }}\right] \text {. }
$$




\section{Wind Tunnel Experiments}

Wind tunnel experiments were conducted to develop the database necessary for detailed sizing and deployment studies of the candidate configurations. The test was conducted during November of 1995 at the Wichita State University (WSU) Walter H. Beech Memorial 7 x 10 foot low-speed wind tunnel [9]. The tunnel is a closed-return design capable of test section dynamic pressures of up to $2873 \mathrm{~Pa}$ (60 psf). Although the experiments were similar to those reported in References 3 and 4, the new tests included several variations and improvements over the previous work, and have substantially contributed to the growing body of data on trailing-edge aerodynamic brakes.

\subsection{Model Design and Instrumentation}

A two-dimensional model was designed and constructed at WSU. The baseline airfoil shape was scaled directly from the $97.5 \%$ radial position of the AWT-26 blade, which is an NREL S810 with a thickened trailing edge. The model had a chord of $45.7 \mathrm{~cm}$ (18 in.) and a nominal length of $2.1 \mathrm{~m}$ $(7 \mathrm{ft})$, which spanned the test section from floor to ceiling. The mounting arrangement allowed an angle of attack range from $-6^{\circ}$ to $+90^{\circ}$.

Figure 4-1 shows a sectional view of the model, with the dashed lines indicating cove inserts that were bolted between the forward and rear model elements. With a cove insert bolted to the forward element, the flap portion of the model was $38 \%$ of the airfoil chord. Alternately, a cove insert could be bolted into the rear portion of the model, resulting in a $45 \%$ chord flap. Either size flap could then be pivoted around any of the hinge positions designated A-1 through C-5. The modular design allowed 30 different flap configurations, including plain unvented flaps, plain vented flaps, and spoiler-flaps.

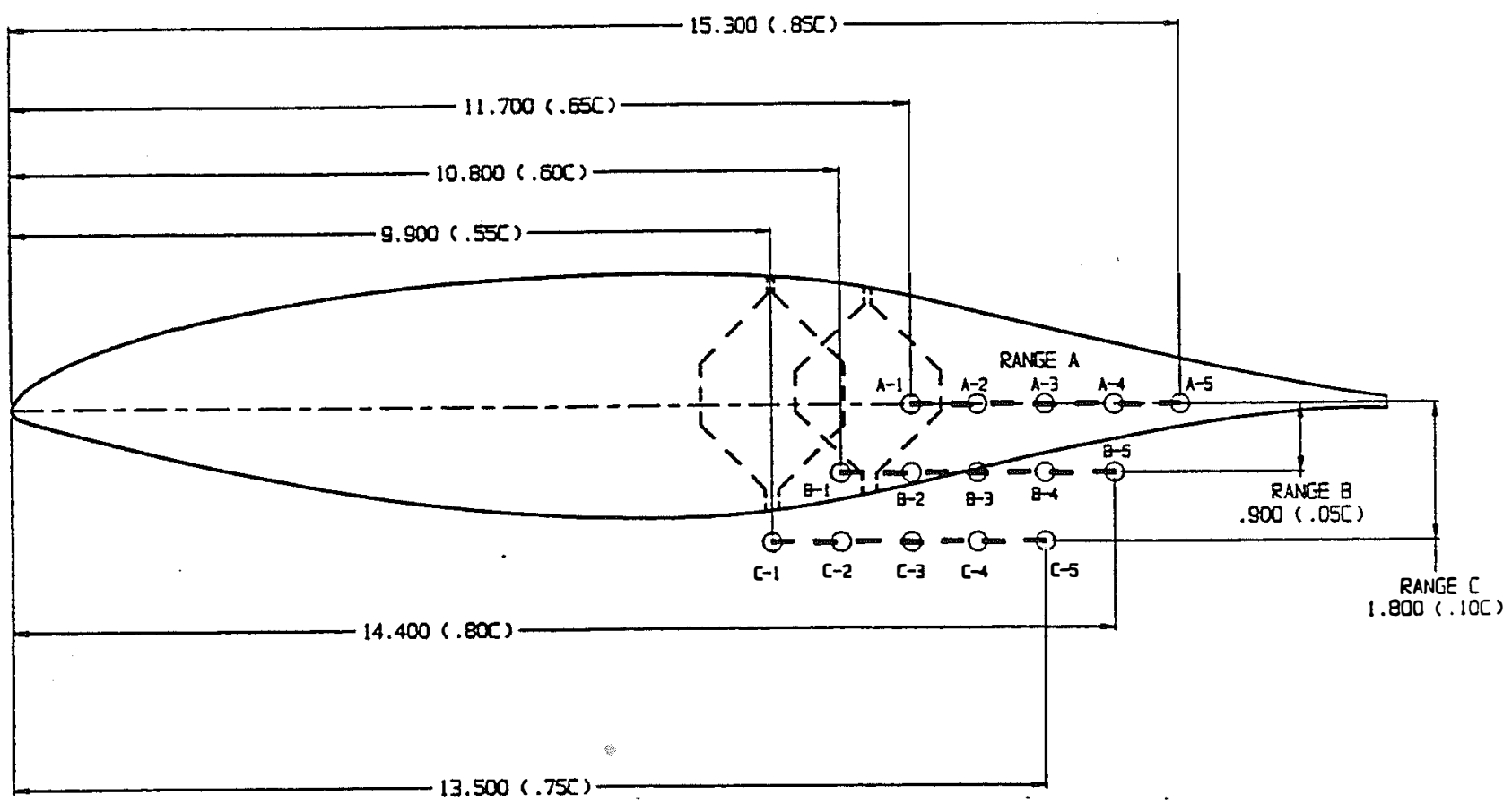

Figure 4-1. Section View of Wind Tunnel Model (primary dimensions in inches) 
The model was supported at the floor and ceiling by balances that measured the lift, drag, and quarterchord pitching moment on the model. The floor balance recorded approximately half of the lift and drag forces, and all of the moment data. The remaining lift and drag forces were resolved by the ceiling balance. Additional documentation on WSU two-dimensional force balances can be found in Reference 9. End plates of $68.6 \mathrm{~cm}$ (27 in.) diameter and $6.4 \mathrm{~mm}(0.25 \mathrm{in}$.) thickness were mounted on each end of the model, approximately $9.5 \mathrm{~mm}(0.375 \mathrm{in}$.) from tunnel walls. The end plates had rounded edges to minimize flow disturbance.

To deflect the flaps, an electronically-controlled actuator assembly was designed and constructed at WSU. As shown in Figure 4-2, the actuator assembly was below the tunnel floor. The device deflections were driven through a flexure (linkage designed to support only axial loads). Hinge moments were measured by the use of strain gages, which were mounted on the flexure and calibrated in-situ during the model installation. The calibration was checked periodically throughout the test by repeat runs of selected configurations. To convert to coefficient form, hinge moment data were normalized with respect to device (flap) chord:

$$
\mathrm{C}_{\mathrm{h}}=\frac{\text { Device Hinge Moment }}{\mathrm{q} \cdot \mathrm{b} \cdot \mathrm{c}_{\text {Device }}^{2}} \text {, }
$$

where $b$ is the airfoil span, and opening hinge moments are defined as positive (trailing-edge down).

Standard wind-tunnel corrections were applied during the data reduction [9]. No attempt was made to measure or correct for turntable interference or tare drag. The authors of Reference 3 used wake deficit measurements to evaluate interference and tare drag, and applied a constant value of $\Delta C_{D}=0.020$ for turntables of similar dimensions. In the present work it was decided to avoid the expense of conducting wake deficit surveys. The data were intended to be used in an incremental fashion and the tare drag measurements would have been of no practical use.

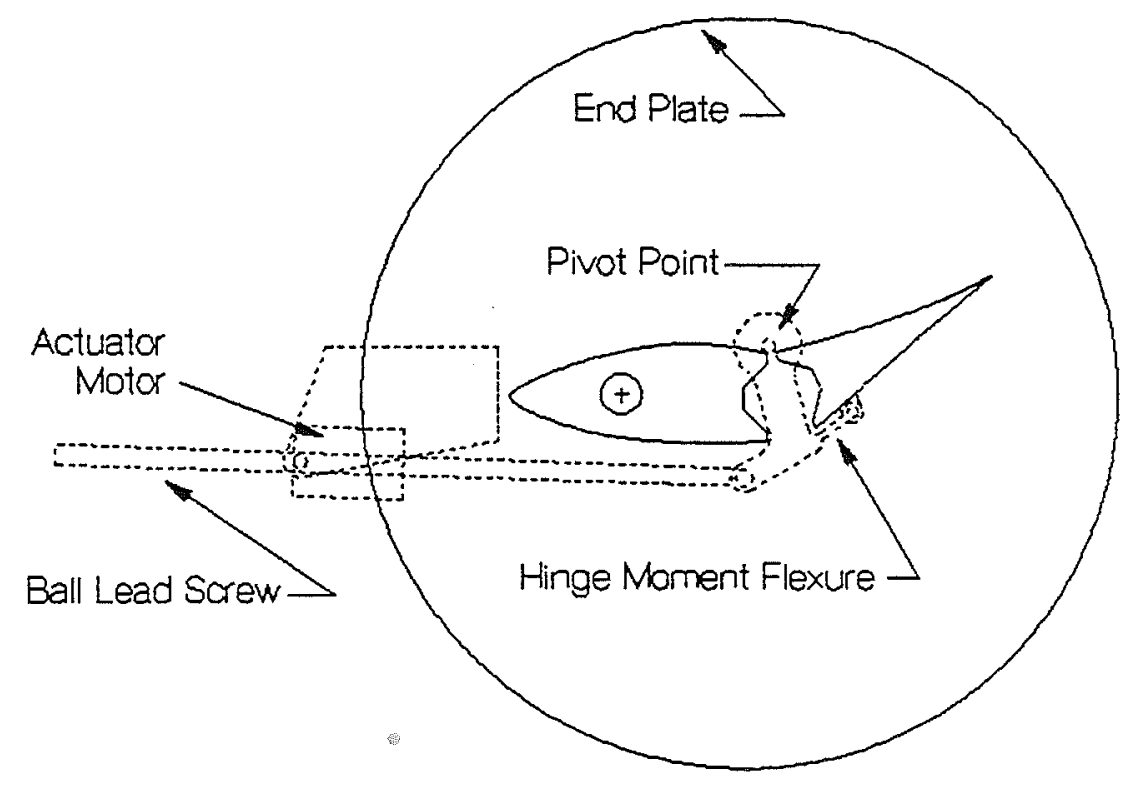

Figure 4-2. End View of Flap Deflection Assembly (dashed lines indicate below floor level) 


\subsection{Test Matrix}

A typical test run was at constant dynamic pressure with a fixed flap-deflection angle and variable angle of attack. For a given flap size and hinge location, a $\delta$-sweep was conducted by test runs performed at a schedule of fixed flap deflections. The resulting data could then be viewed in a matrix form, aerodynamic performance as a function of both $\alpha$ and $\delta$. The majority of runs were conducted at a dynamic pressure of $479 \mathrm{~Pa}(10 \mathrm{psf})$, which corresponds to a Reynolds number of approximately 1.0 million based on the model chord (including standard WSU corrections for turbulence effects). The typical angle of attack range was $-6^{\circ} \leq \alpha \leq 90^{\circ}$. Table 4-1 summarizes the 15 configurations that were tested in the $\delta$-sweep manner.

Table 4-1. Flap Configurations Tested by Standard $\delta$-Sweep

\begin{tabular}{ccll}
\hline $\begin{array}{c}\text { Flap Chord } \\
\text { (\% of airfoil) }\end{array}$ & $\begin{array}{c}\text { Hinge } \\
\text { Location }\end{array}$ & $\begin{array}{c}\text { Configuration } \\
\text { Type }\end{array}$ & \multicolumn{1}{c}{$\begin{array}{c}\text { Flap Deflections Tested } \\
\text { (degrees) }\end{array}$} \\
\hline 38 & A-2 & Plain Flap & $0,15,30,45,60,90$ \\
38 & A-3 & Spoiler-Flap & $0,5,10,30,60,90$ \\
38 & A-4 & Spoiler-Flap & $0,5,10,20,30,40,60,75,90$ \\
38 & A-5 & Spoiler-Flap & $0,5,10,20,30,40,60,75,90$ \\
38 & B-1 & Plain Flap & $0,15,30,45,60,75,90$ \\
38 & C-2 & Vented Flap & $0,15,30,45$ \\
45 & A-1 & Plain Flap & $0,15,30,45,60,75,90$ \\
45 & A-2 & Spoiler-Flap & $0,15,30,45,60,75,90$ \\
45 & A-3 & Spoiler-Flap & $0,15,30,45,60,75,90$ \\
45 & A-4 & Spoiler-Flap & $0,5,10,15,30,45,60,75,90$ \\
45 & B-3 & Spoiler-Flap & $0,15,30,45,60,75,90$ \\
45 & B-5 & Spoiler-Flap & $0,15,30,45,60,75$ \\
45 & C-1 & Vented Flap & $0,15,30,45,60,75,90$ \\
45 & C-3 & Spoiler-Flap & $0,15,30,45,60,85$ \\
45 & C-5 & Spoiler-Flap & $0,15,30,45,60$ \\
\hline
\end{tabular}

The determination of device type was in some cases subjective. For instance, the $45 \%$ chord device at hinge location A-1 was clearly a plain flap, and at hinge A-3 it was clearly a spoiler-flap, but hinge A-2 was somewhat in between. The device was labeled as a spoiler-flap if a significant portion of the device leading edge protruded above the upper-airfoil (low-pressure) surface as the flap was deployed. Where plain flaps showed significant venting at low deployment angles, they have been labeled as "vented flaps." Inspection of Table 4-1 shows that the $\delta$-sweeps generally emphasized spoiler-flaps, and more tests were conducted for the $45 \%$ chord device than for the $38 \%$. The test matrix shown in Table 4-1 bracketed the parameter space of interest, and provided an excellent database from which to predict the aerodynamic performance of both the spoiler-flap and flip-tip.

In addition to the configurations shown in Table 4-1, several special studies were conducted, including tests to determine the effects of Reynolds number, device gap leakage, leading-edge roughness, and device aspect ratio. Throughout the test, selected configurations were repeated to ensure that all instruments were holding their calibrations. These special studies will be discussed in greater detail in the following sections.

A total of 165 runs were completed during the test. A complete run log that documents the test configurations and conditions is provided in Appendix A, along with graphical data for 119 of the test runs. An ASCII text file of the complete data set is available from NREL or WSU by request. 


\subsection{Wind Tunnel Test Results and Discussion}

\subsubsection{Baseline S810 Airfoil}

As shown in Figure 4-1, the forward and rear elements of the airfoil model were separated by gaps with an open cavity (cove) in between. The gap dimensions were $0.5 \%$ chord on the low-pressure side of the airfoil, and $1.0 \%$ chord on the high-pressure side. During all standard wind-tunnel runs these gaps were unsealed, resulting in a surface discontinuity and allowing leakage from the high-to lowpressure surfaces.

To assess the impact on airfoil drag, special runs were conducted with the gaps taped. Figures 4-3 and 4-4 show the results of these tests, and indicate a significant drag penalty when the gaps are open, particularly at the pre-stall angles of $6^{\circ} \leq \alpha \leq 9^{\circ}$. The effect is most pronounced for the $45 \%$ chord device, where the gap occurs at the $55 \%$ airfoil chord location. For both devices, drag penalties are in the range of 40 to 100 drag counts over a large portion of the drag bucket, where a drag count is an increment of 0.0001 in drag coefficient. For the $S 810$ airfoil at 1.0 million Reynolds number, an increase of 10 drag counts represents a $14 \%$ increase relative to the clean airfoil drag.

Full lift, drag, and suction coefficient curves for the baseline S810 airfoil are shown in Figures 4-5 and 4-6 of the following sections, as they represent the $\delta=0^{\circ}$ case for each flap configuration tested. The data show a maximum lift coefficient of $C_{L_{\max }} \approx 1.0$, which occurs at $\alpha=12^{\circ}$, and a smooth stall progression to $C_{L} \approx 0.7$ at $\alpha \approx 25^{\circ}$. The data show a relatively flat drag bucket between $-6^{\circ} \leq \alpha \leq 10^{\circ}$. As the airfoil approaches stall, the drag increases rapidly to a value of $C_{D_{\max }} \approx 1.7$ at $\alpha=90^{\circ}$. The positive suction peak of $\mathrm{C}_{S_{\max }} \approx 0.15$ coincides with the maximum lift coefficient at $\alpha=12^{\circ}$.

\subsubsection{5\% Chord Devices}

Aerodynamic coefficients for the $45 \%$ plain flap are shown in Figures $4-5$, where the hinge location is A-1. The curves show typical flap behavior, with $C_{L \alpha=0}$ and $C_{L_{\max }}$ both strictly increasing with flap deflections up to $\delta=75^{\circ}$. Although the flap acts as a high-lift device, it also produces significant drag, and the suction coefficients remain negative for all flap deflections greater than $15^{\circ}$. Hinge moments, which are strongly negative (closing), increase smoothly with both flap deflection and airfoil angle of attack.

Figures 4-6 show aerodynamic coefficients for a $45 \%$ chord spoiler-flap hinged at A-3. At moderate deflection angles the spoiler-flap exhibits some high-lift behavior, particularly at post-stall airfoil angles of attack. However, for the spoiler deployed to $75^{\circ}$ or greater, the lift is greatly diminished at all angles of attack. The effect of lift spoiling on suction coefficients is most apparent at angles of attack near the clean-airfoil suction peak $\left(5^{\circ}<\alpha<15^{\circ}\right)$. A comparison of Figures 4-5c and 4-6c shows that for angles less than $\alpha \approx 20^{\circ}$, the fully deployed spoiler-flap has suction coefficients which are approximately $20 \%$ more negative than those for the plain flap. Above $\alpha \approx 25^{\circ}$, the plain flap becomes considerably more effective. Hinge location A-3 is very close to neutral in terms of hinge moments. Figure 4-6d shows that hinge moment coefficients are nearly invariant with angle of attack, and remain near zero or slightly negative (closing) over most deflection angles.

Figures 4-7 show the variation of $45 \%$ chord devices with hinge location at a fixed deflection angle of $\delta=75^{\circ}$. Hinge location $A-1$ is a plain unvented flap, location $C-1$ is a plain vented flap, and the remainder of the locations are spoiler-flaps. This is particularly obvious in the lift curves of 


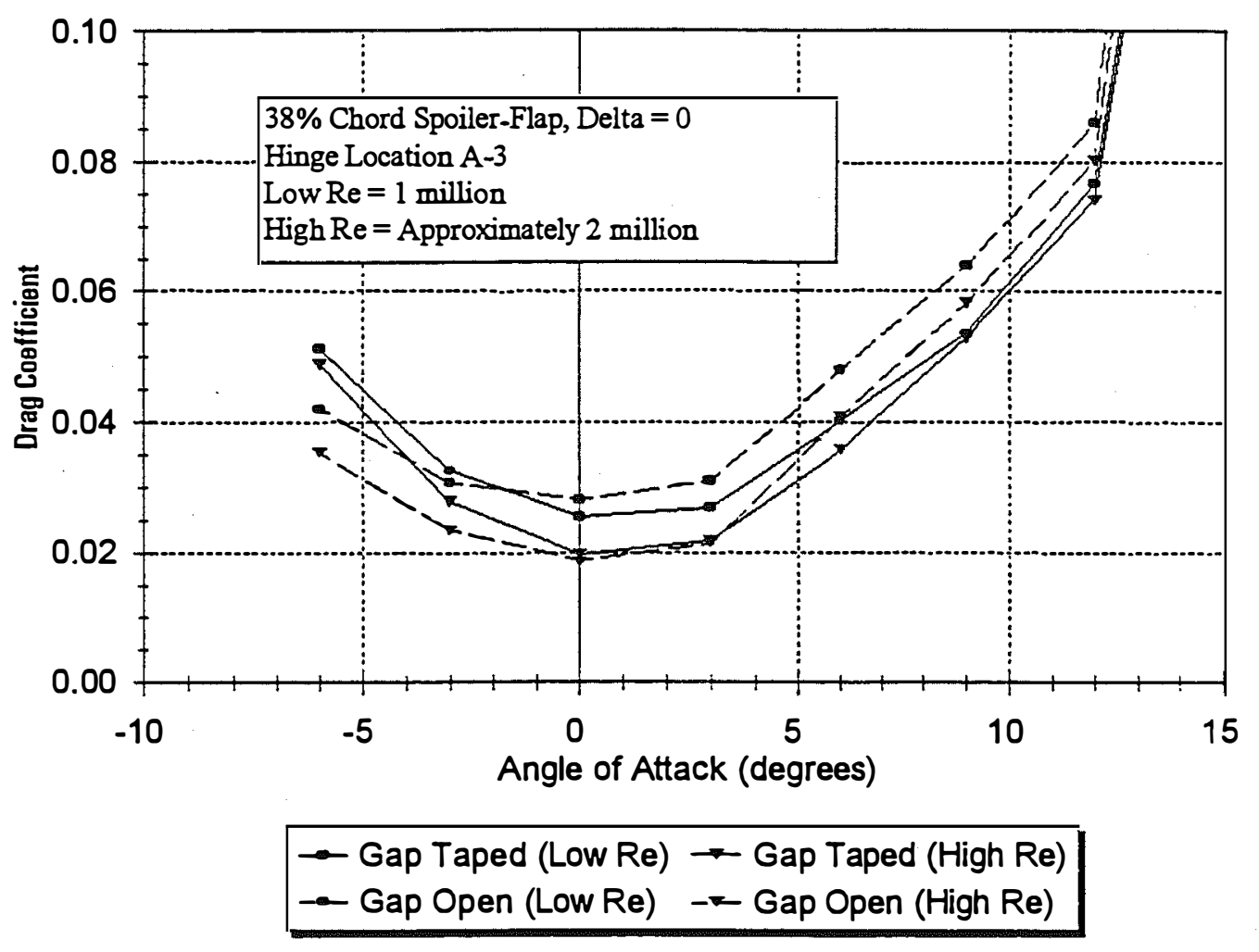

Figure 4-3. Effect of Gap Seal on 38\% Chord Device

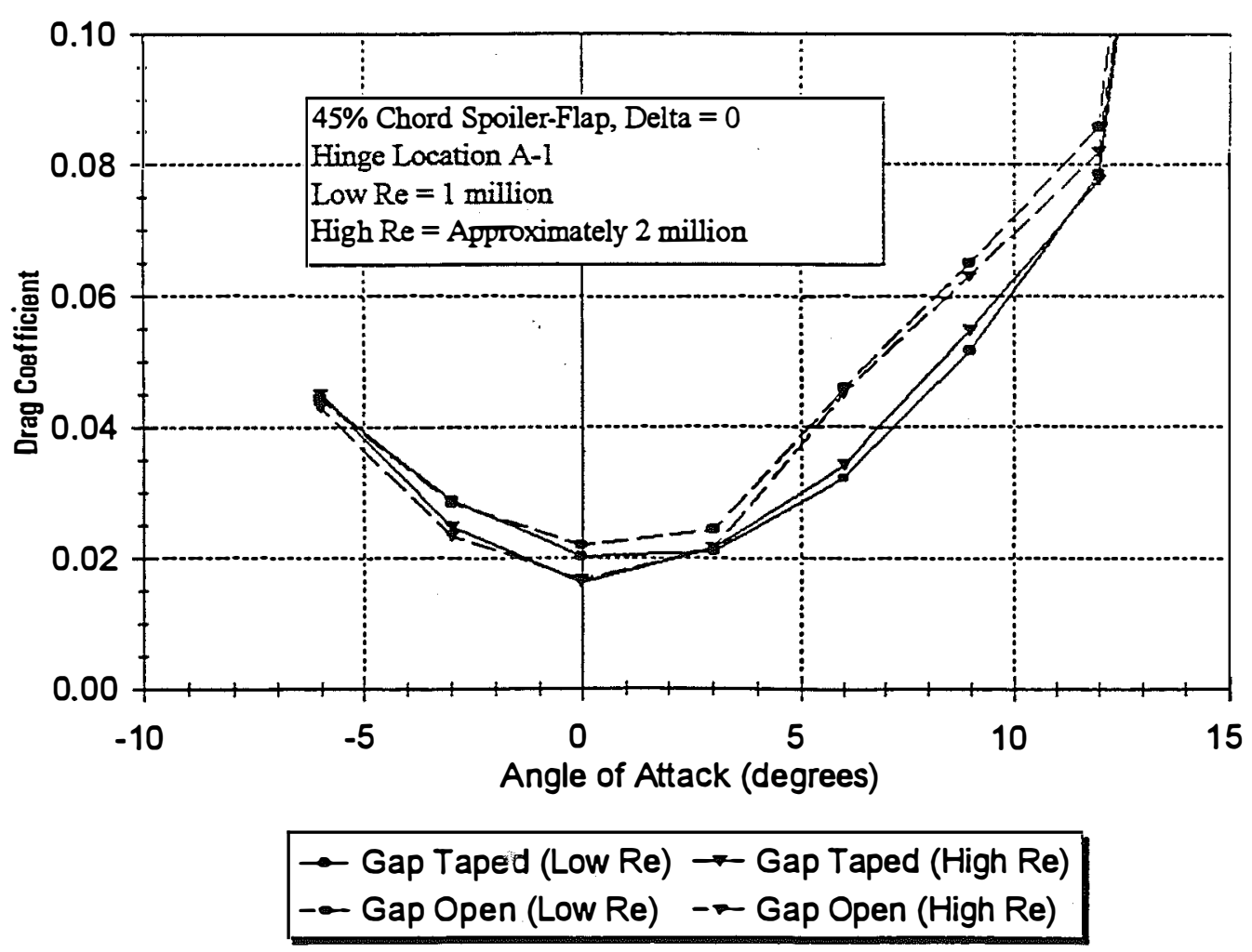

Figure 4-4. Effect of Gap Seal on 45\% Chord Device 


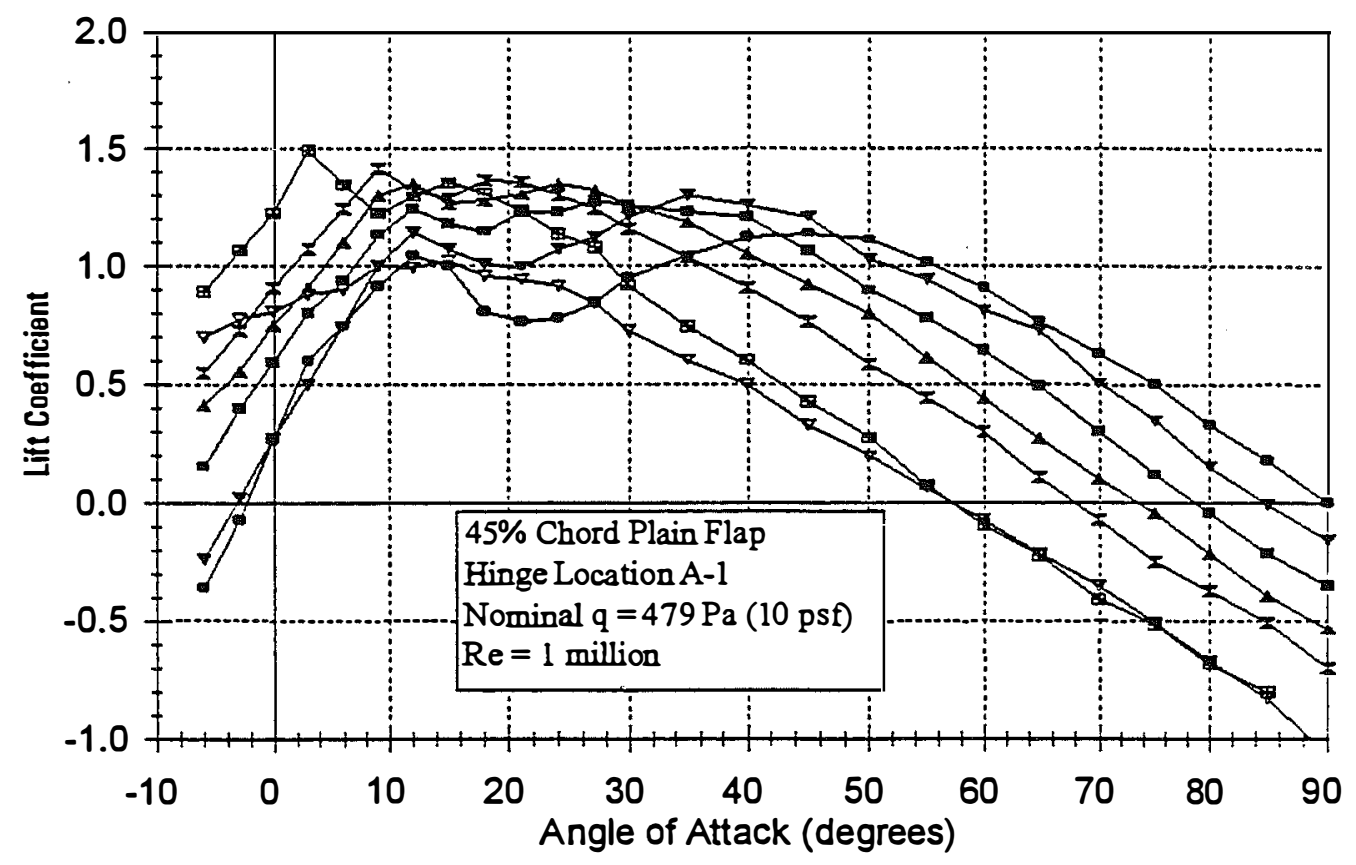

$$
\begin{aligned}
& \rightarrow \text { Delta }=0 \rightarrow \text { Delta }=15 \rightarrow \text { Delta }=30 \rightarrow \text { Delta }=45 \\
& \rightarrow \text { Delta }=60 \rightarrow \text { Delta }=75 \rightarrow \text { Delta }=90
\end{aligned}
$$

Figure 4-5a. Lift Coefficients for $45 \%$ Chord Plain Flap, Hinge A-1

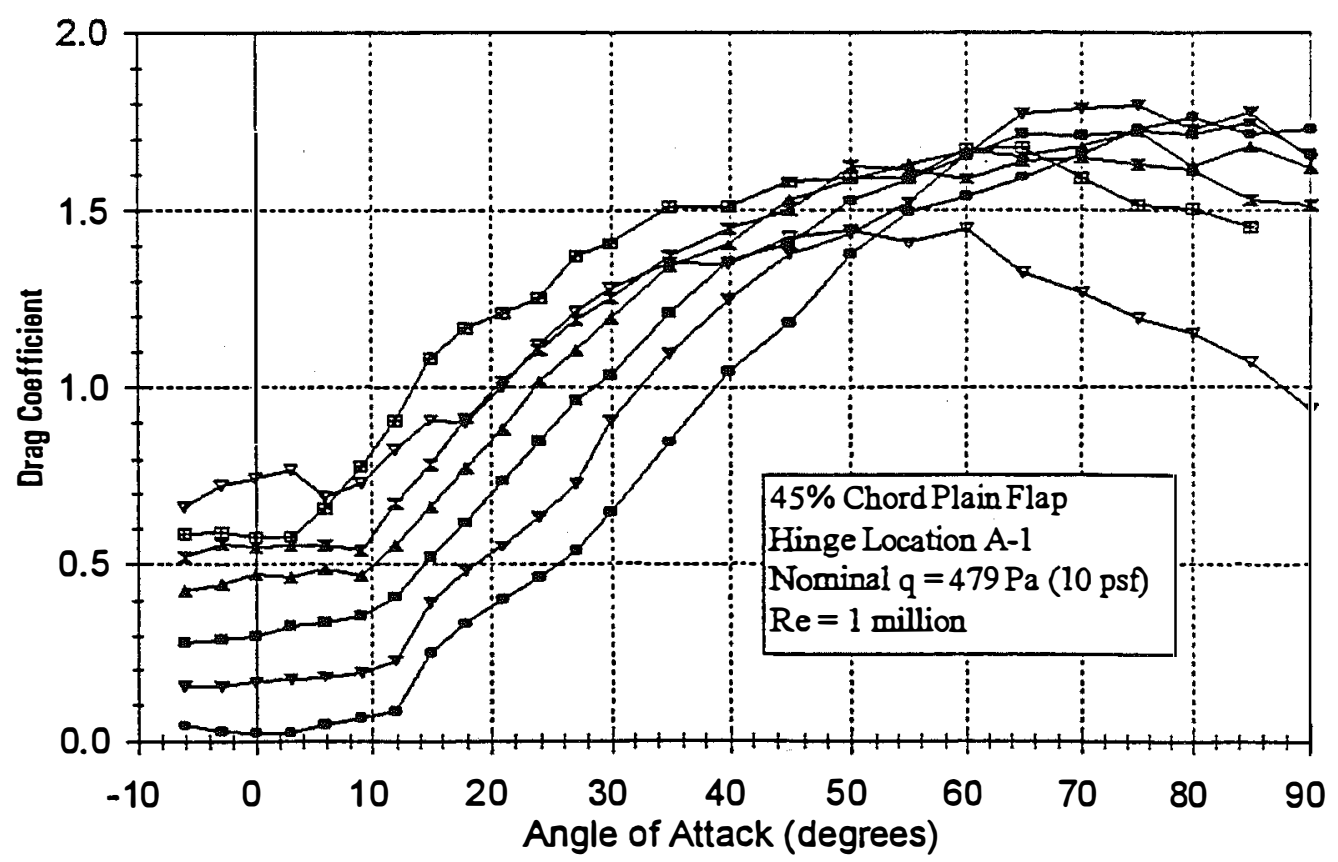

$$
\begin{aligned}
& \rightarrow \text { Delta }=0 \rightarrow \text { Delta }=15 \rightarrow \text { Delta }=30 \rightarrow \text { Delta }=45 \\
& \rightarrow \text { Delta }=60 \rightarrow \text { Delta }=75 \rightarrow \text { Delta }=90
\end{aligned}
$$

Figure 4-5b. Drag Coefficients for $\mathbf{4 5 \%}$ Chord Plain Flap, Hinge A-1 


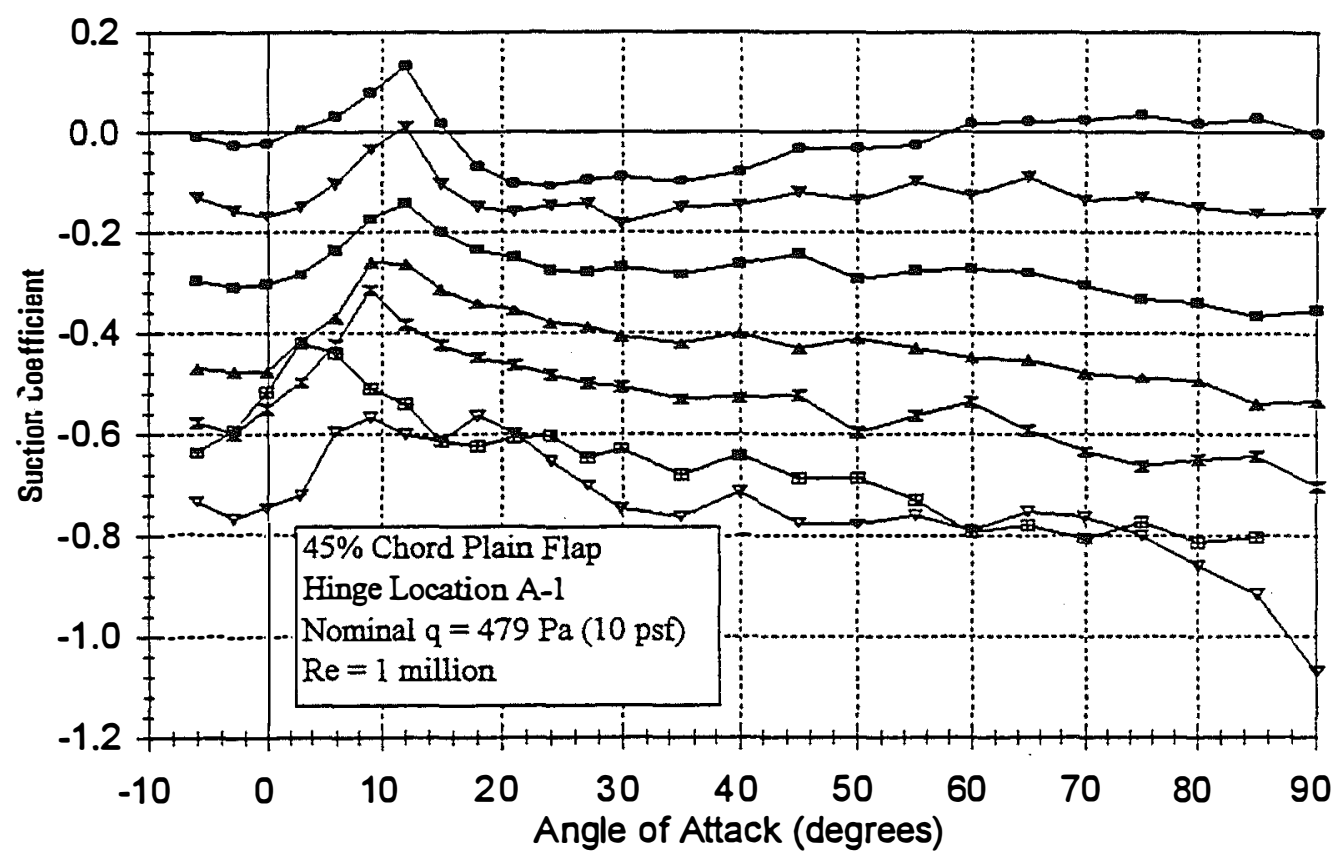

$$
\begin{aligned}
& \rightarrow \text { Delta }=0 \rightarrow \text { Delta }=15 \rightarrow \text { Delta }=30 \rightarrow \text { Delta }=45 \\
& \rightarrow \text { Delta }=60 \rightarrow \text { Delta }=75 \rightarrow \text { Delta }=90
\end{aligned}
$$

Figure 4-5c. Suction Coefficients for $45 \%$ Chord Plain Flap, Hinge A-1

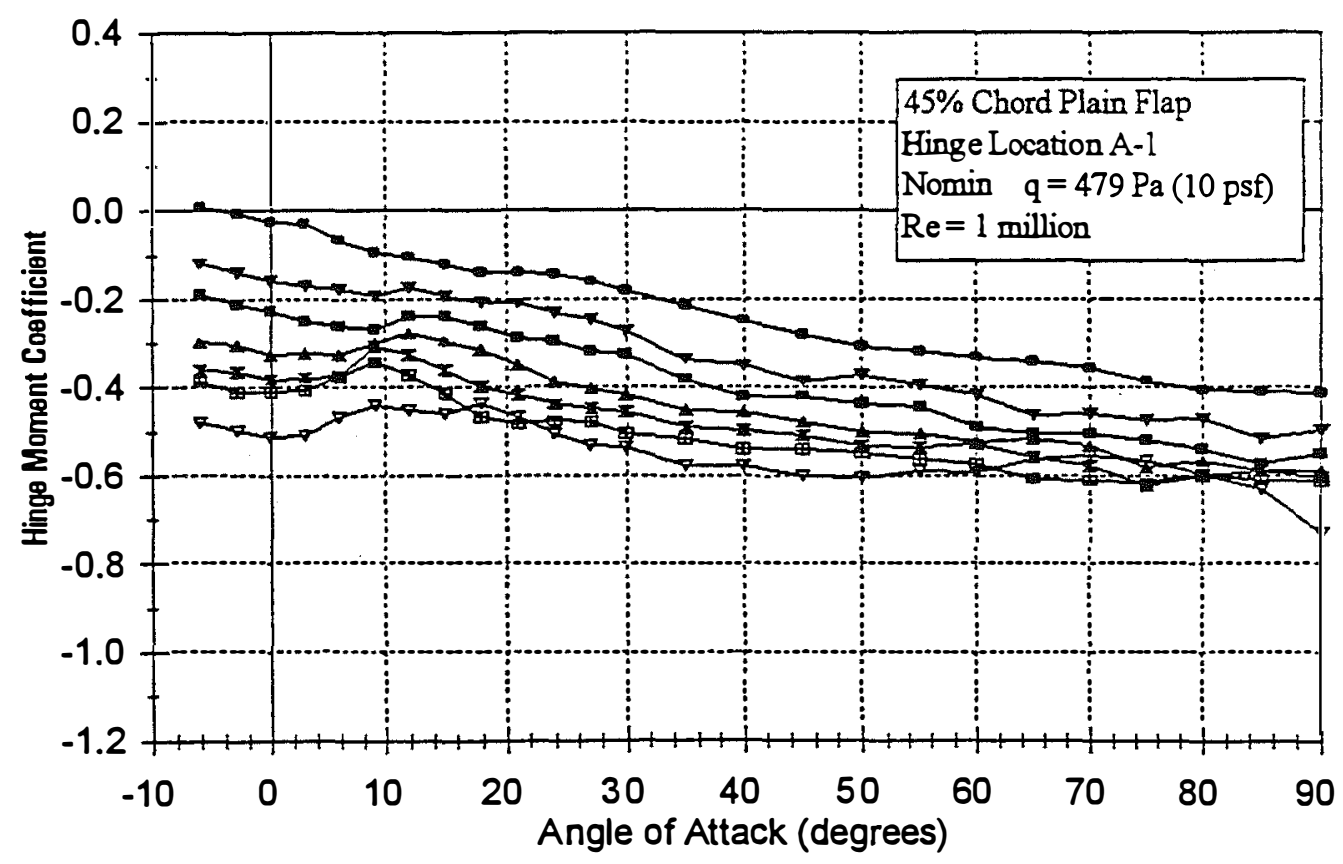

$$
\begin{aligned}
& \rightarrow \text { Delta }=0 \rightarrow \text { Delta }=15 \rightarrow \text { Delta }=30 \rightarrow \text { Delta }=45 \\
& \rightarrow \text { Delta }=60 \rightarrow \text { Delta }=75 \rightarrow \text { Delta }=90
\end{aligned}
$$

Figure 4-5d. Hinge Moment Coefficients for $45 \%$ Chord Plain Flap, Hinge A-1 


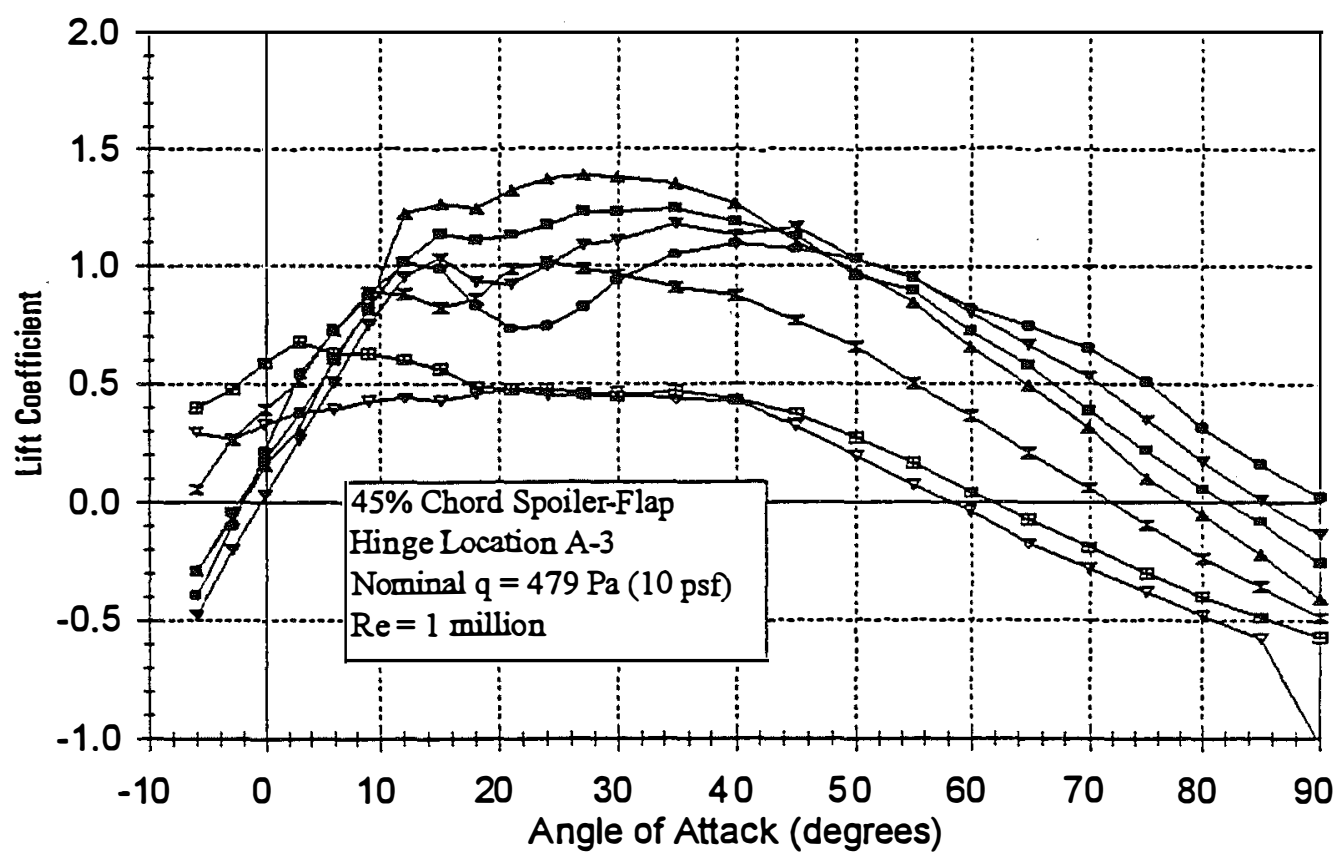

$$
\begin{aligned}
& \rightarrow \text { Delta }=0 \rightarrow \text { Delta }=15 \rightarrow \text { Delta }=30 \multimap \text { Delta }=45 \\
& \rightarrow \text { Delta }=60 \rightarrow \text { Delta }=75 \rightarrow \text { Delta }=90
\end{aligned}
$$

Figure 4-6a. Lift Coefficients for $\mathbf{4 5 \%}$ Chord Spoiler-Flap, Hinge A-3

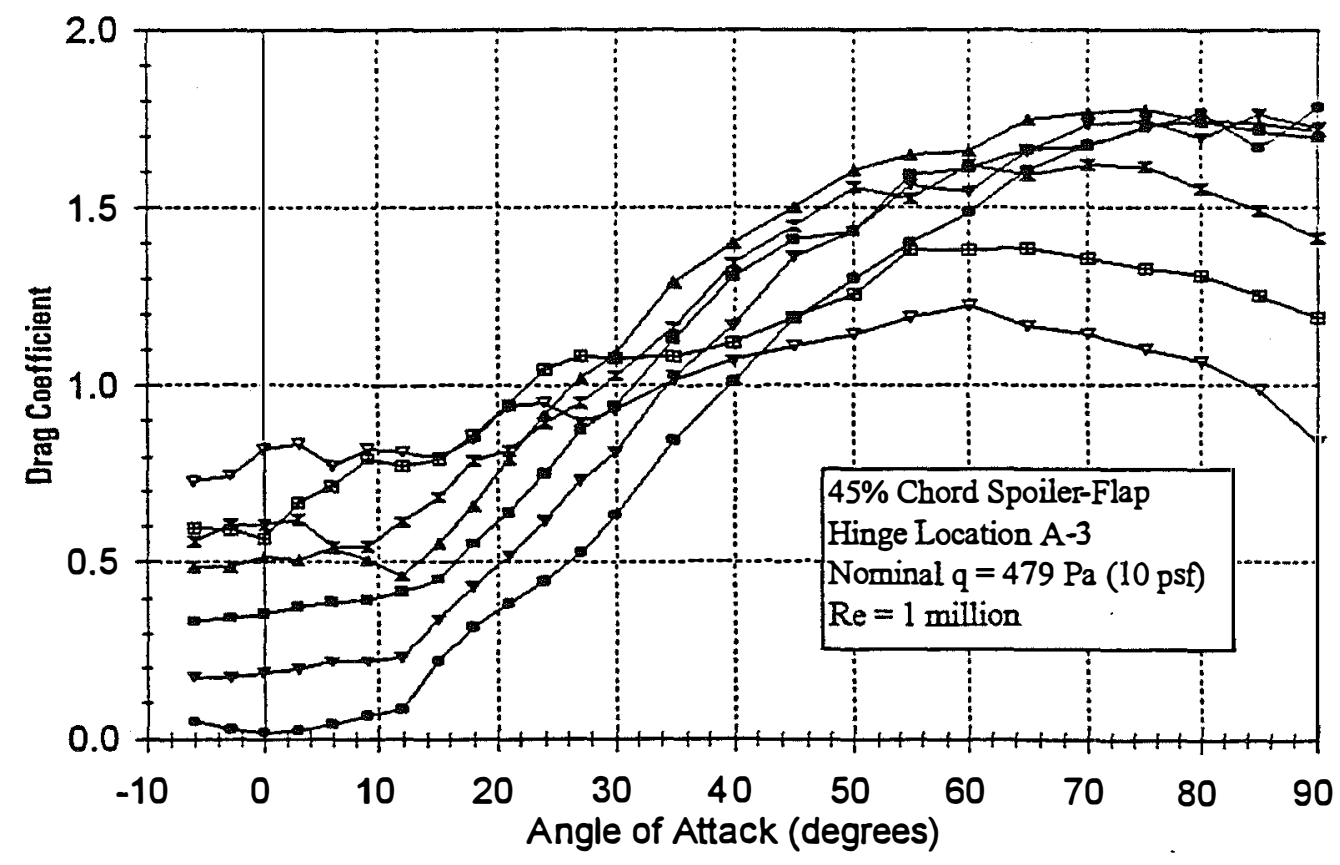

$$
\begin{aligned}
& \rightarrow \text { Delta }=0 \rightarrow \text { Delta }=15 \rightarrow \text { Delta }=30 \rightarrow \text { Delta }=45 \\
& \rightarrow \text { Delta }=60 \rightarrow \text { Delta }=75 \rightarrow \text { Delta }=90
\end{aligned}
$$

Figure 4-6b. Drag Coefficients for $\mathbf{4 5 \%}$ Chord Spoiler-Flap, Hinge A-3 


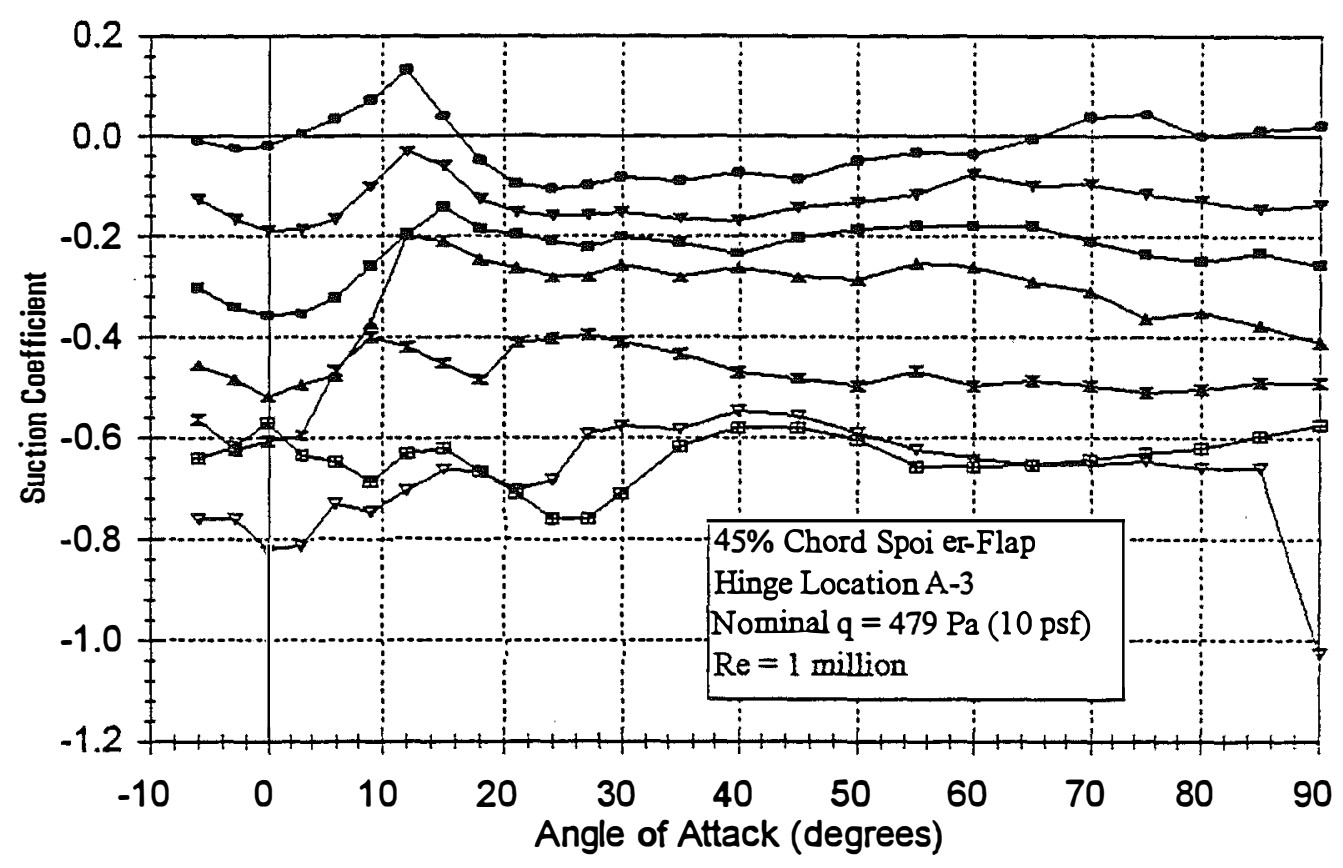

$$
\begin{aligned}
& \rightarrow \text { Delta }=0 \rightarrow \text { Delta }=15 \rightarrow \text { Delta }=30 \rightarrow \text { Delta }=45 \\
& \rightarrow \text { Delta }=60 \rightarrow \text { Delta }=75 \rightarrow \text { Delta }=90
\end{aligned}
$$

Figure 4-6c. Suction Coefficients for $45 \%$ Chord Spoiler-Flap, Hinge A-3

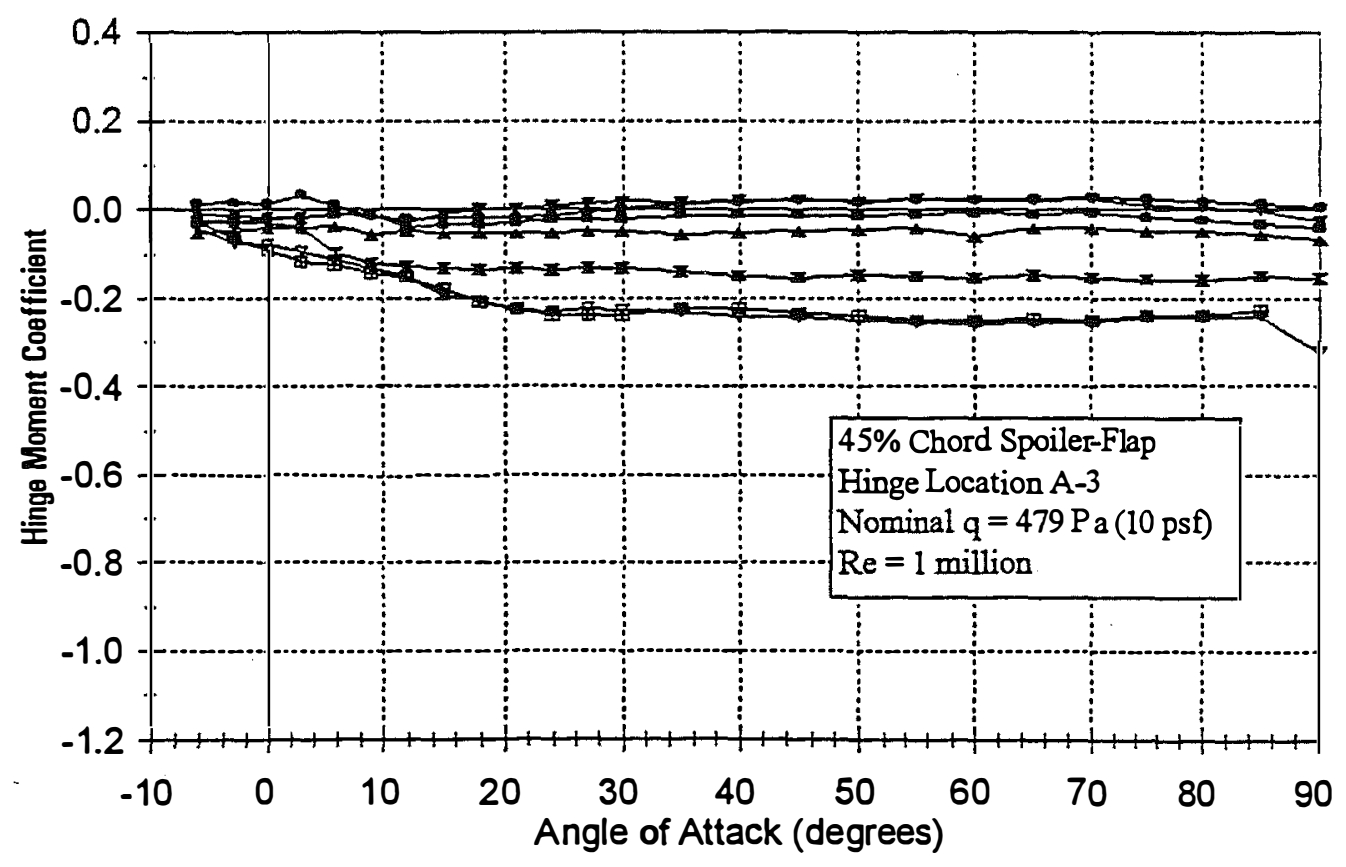

$\rightarrow$ Delta $=0 \rightarrow$ Delta $=15 \rightarrow$ Delta $=30 \rightarrow$ Delta $=45$

$\rightarrow$ Delta $=60 \rightarrow$ Delta $=75 \rightarrow$ Delta $=90$

Figure 4-6d. Hinge Moment Coefficients for $45 \%$ Chord Spoiler-Flap, Hinge A-3 


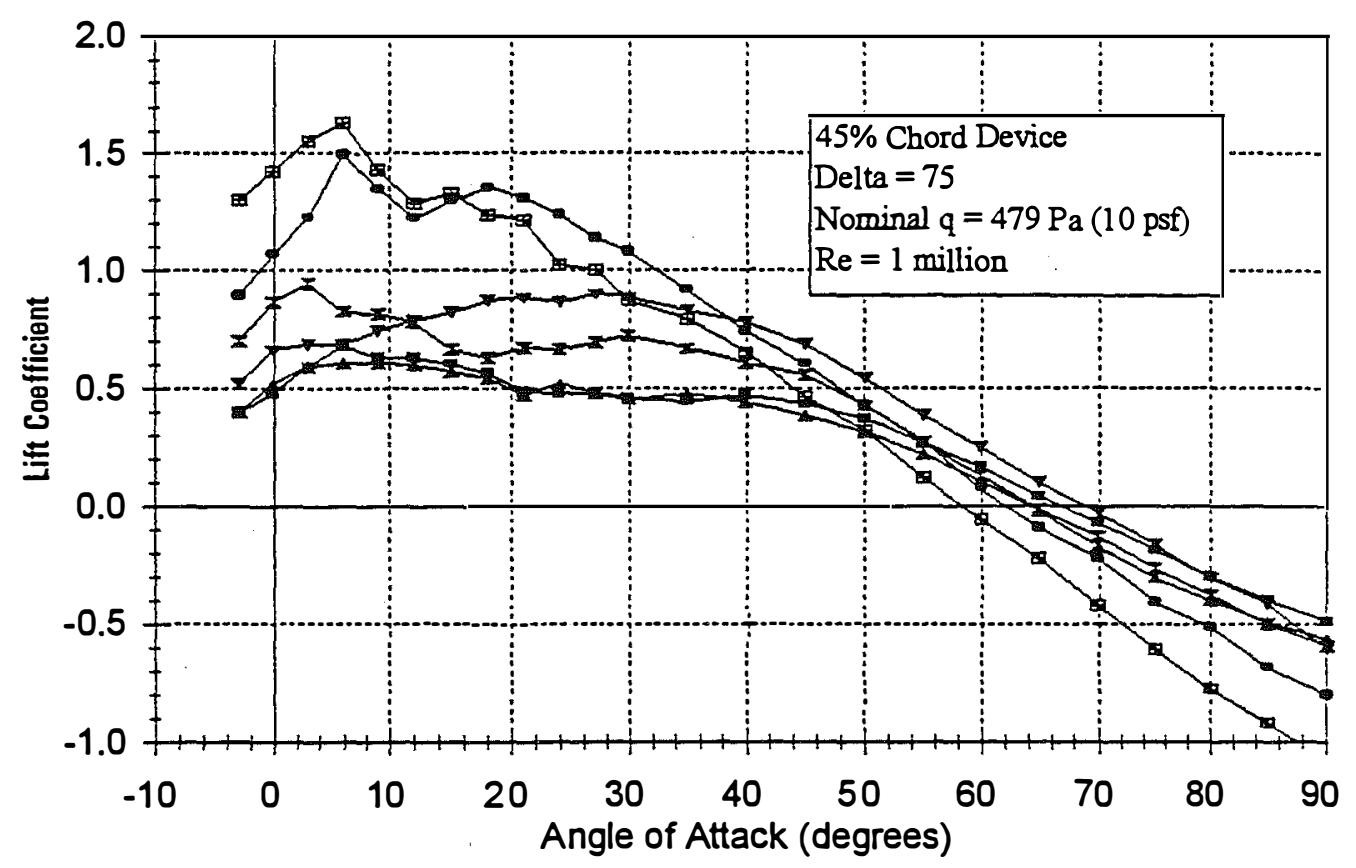

$\rightarrow$ Hinge A-1 $\rightarrow$ Hinge A-2 $\rightarrow$ Hinge A-3
- Hinge A-4 $\rightarrow$ Hinge B-3 $\rightarrow$ Hinge C-1

Figure 4-7a. Lift Data, Effect of Hinge Location on Device at $\delta=75^{\circ}$

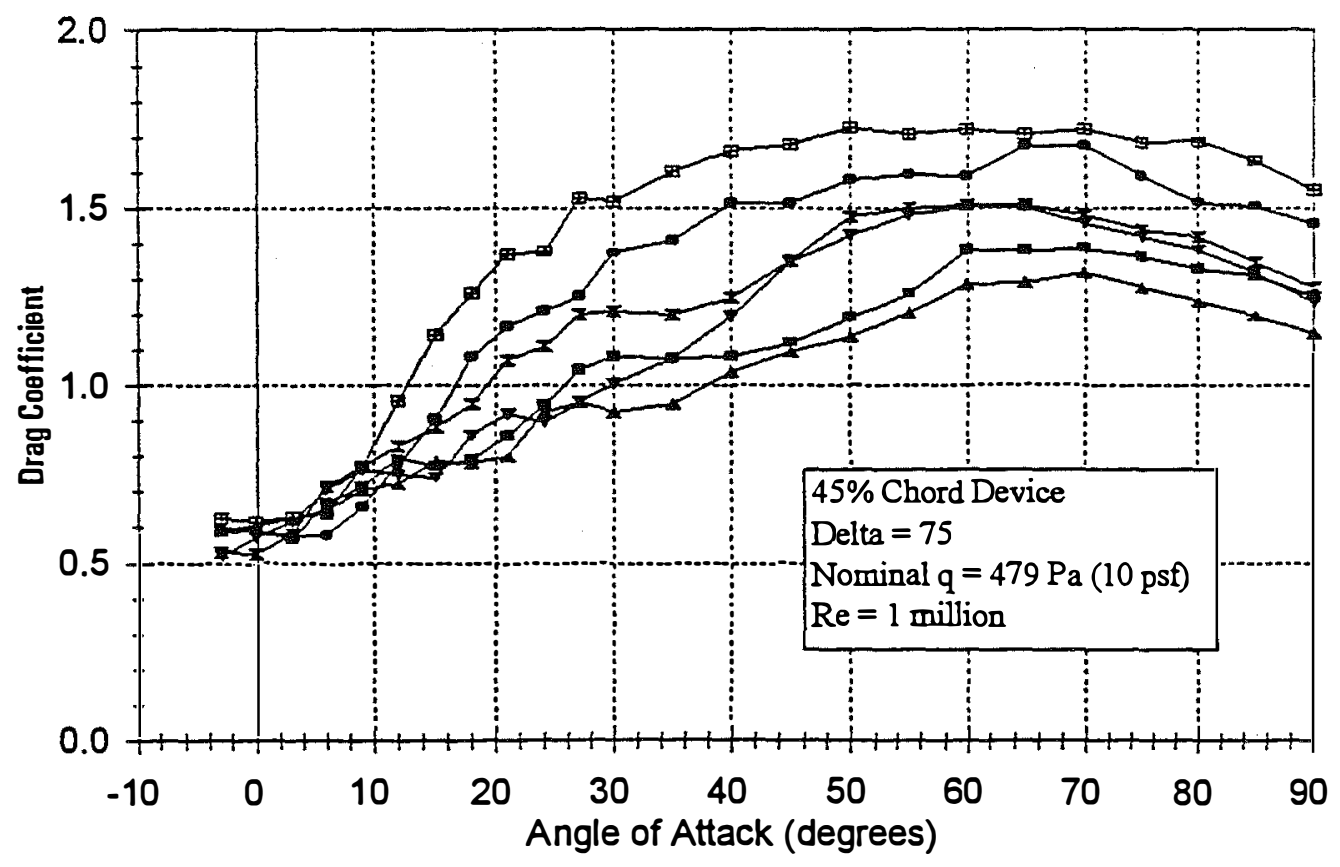

$\rightarrow$ Hinge A-1 $\rightarrow$ Hinge A-2 $\rightarrow$ Hinge A-3
$\rightarrow$ Hinge A-4 - Hinge B-3 $\rightarrow$ Hinge C-1

Figure 4-7b. Drag Data, Effect of Hinge Location on Device at $\delta=75^{\circ}$ 


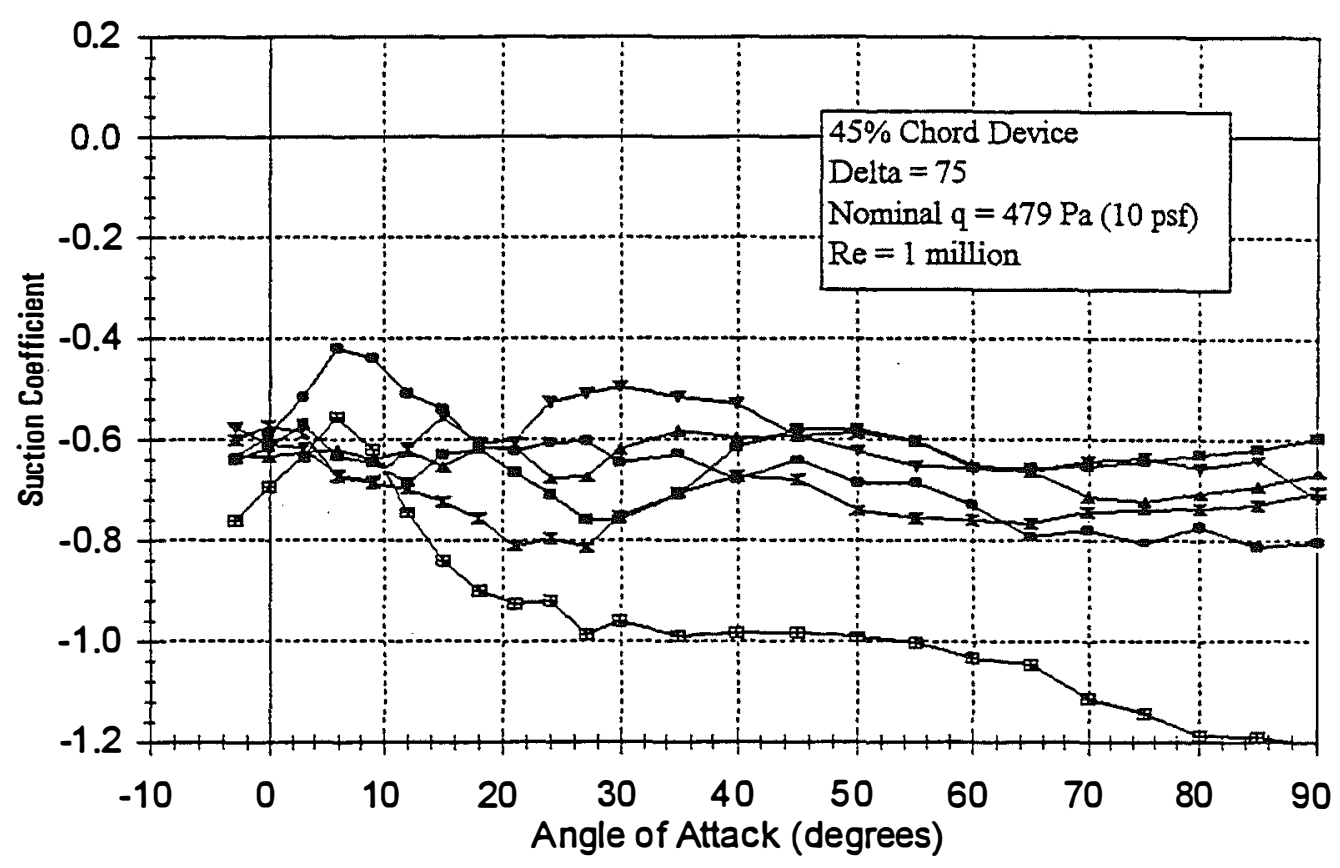

$\rightarrow$ Hinge A-1 $\rightarrow$ Hinge A-2 $\rightarrow$ Hinge A-3
- Hinge A-4 $\rightarrow$ Hinge B-3 $\rightarrow$ Hinge C-1

Figure 4-7c. Suction Data, Effect of Hinge Location on Device at $\delta=75^{\circ}$

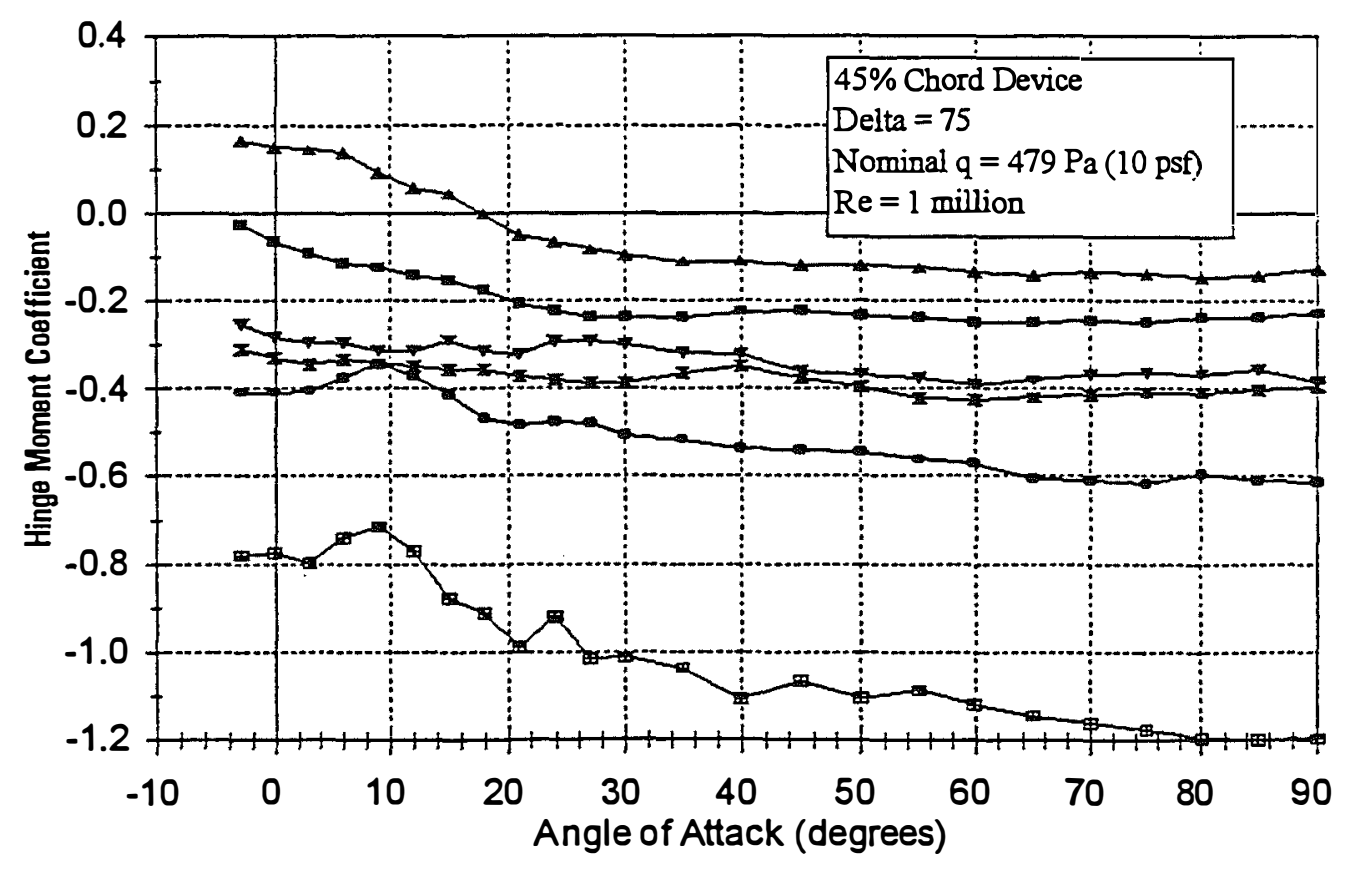

$\rightarrow$ Hinge A-1 $\rightarrow$ Hinge A-2 $\rightarrow$ Hinge A-3
$\rightarrow-$ Hinge $A-4 \rightarrow-$ Hinge $B-3 \rightarrow$ Hinge C-1

Figure 4-7d. Hinge-Moment Data, Effect of Hinge Location on Device at $\delta=75^{\circ}$ 
Figure 4-7a, where the plain and vented flaps show significant high-lift behavior. In terms of suction coefficient, the vented flap (hinge C-1) exhibits the largest variation with angle of attack, showing a noticeable suction peak at $\alpha=9^{\circ}$ and large negative suction coefficients at $\alpha>20^{\circ}$. At pre-stall angles of attack, the spoiler-flap configurations show little variation of suction coefficient with hinge location, with values generally ranging between -0.6 and -0.7 .

Figure 4-7d shows that the vented flap has very high closing hinge moments. Both the force and hingemoment data for the vented flap are consistent with the fact that the hinge position allows larger aerodynamic surfaces to be exposed to the free-stream air than for the other hinge points considered. At $\delta=75^{\circ}$, the spoiler-flap data show smoothly varying hinge moment trends, with an increasing tendency towards opening as the devices are hinged further aft.

\subsubsection{Effect of Device Chord}

A limited discussion of the effect of varying device chord is presented here, with the complete set of aerodynamic data for the $38 \%$ chord devices available in Appendix A. As might be expected, the $38 \%$ chord devices generally resulted in smaller changes to the aerodynamic forces and smaller hinge moments. However, Figure 4-8 shows the effectiveness per unit device chord to be quite similar. The figure shows changes in suction coefficient for both the $38 \%$ and $45 \%$ chord devices, where the quantity plotted is:

$\frac{\Delta \mathrm{C}_{\mathrm{S}}}{\text { Unit Device Chord }}=\frac{\mathrm{C}_{\mathrm{S}, \text { device deflected }}-\mathrm{C}_{\mathrm{S}, \text { clean aiffoil }}}{\text { (Device chord / Aiffoil Chord })}$.

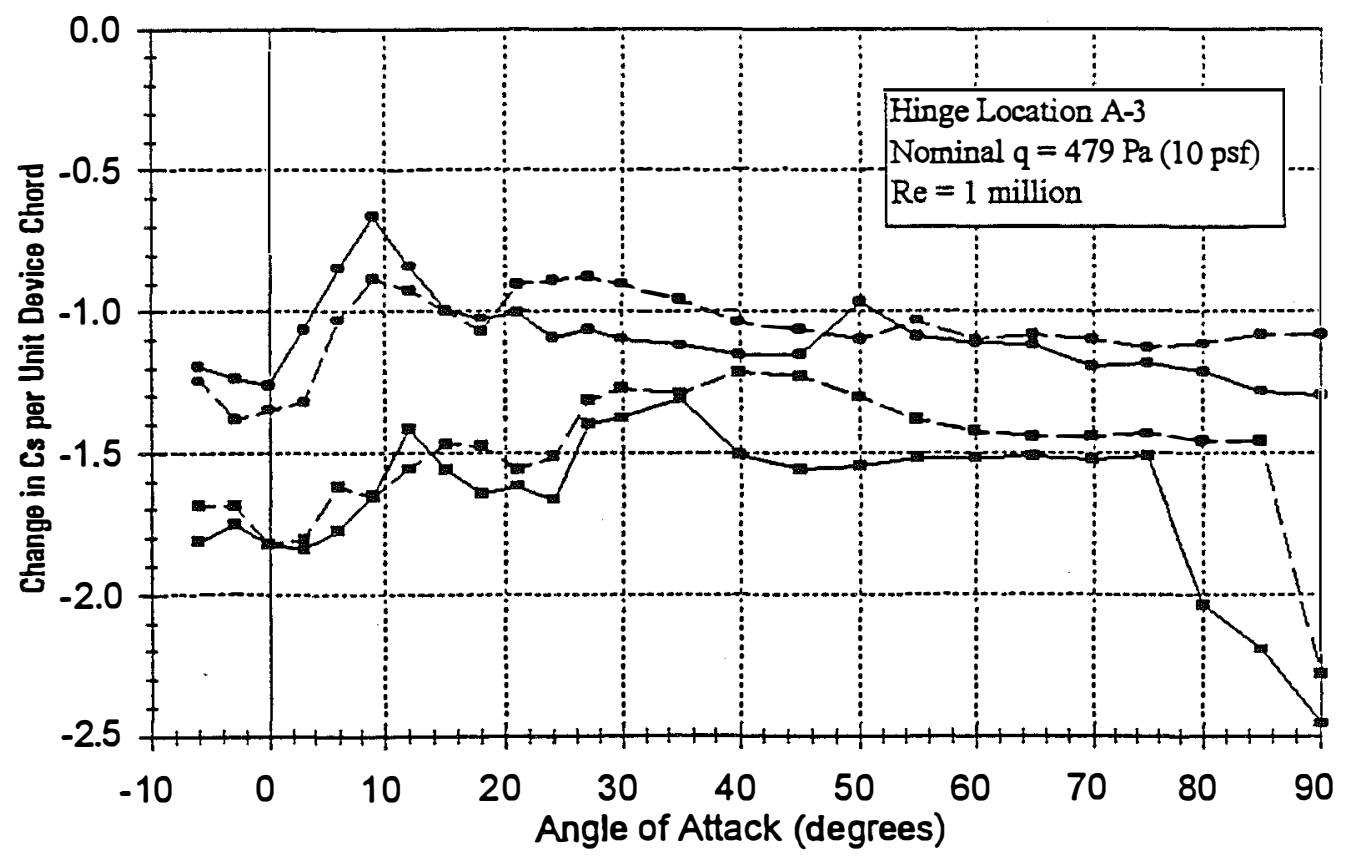

$\rightarrow 38 \%$ Chord Device, Delta $=60-45 \%$ Chord Device, Delta $=60$

$38 \%$ Chord Device, Delta $=90--45 \%$ Chord Device, Delta $=90$

Figure 4-8. Braking Effectiveness per Unit Device Chord 


\subsubsection{Reynolds Number Effects}

As discussed in Section 4.2, the majority of wind-tunnel test runs were conducted at a nominal Reynolds number of 1.0 million. This is significantly lower than the operational Reynolds numbers of the AWT-26/27 turbines, which range between 2.0 and 3.0 million in the blade-tip regions. Selected runs were therefore conducted at high Reynolds number to confirm that the wind tunnel data could be used to predict the full-scale performance of devices.

During the wind tunnel tests, maximum Reynolds numbers were generally constrained by balance limits. With flaps deflected, running at high dynamic pressures resulted in large side loads (in the standard wind tunnel frame of reference). To obtain high Reynolds number data, the tunnel operators would maintain the highest possible dynamic pressure without exceeding any balance limits. This resulted in runs where the Reynolds number varied with angle of attack, and the data from these runs must therefore be interpreted with care.

Although the procedure for obtaining high Reynolds number data was somewhat cumbersome, the objective was satisfied. At pre-stall airfoil angles of attack, and at small device deflection angles, the tunnel operators were able to maintain Reynolds numbers of about 2.0 million. At post-stall airfoil angles and at high device deflections the maximum Reynolds numbers were as low as 1.4 million. Therefore, the highest Reynolds numbers were obtained at the conditions for which the greatest effect was expected.

Figures 4-9 shows that the measured effect of Reynolds number on the spoiler-flap performance was fairly subtle. The insensitivity of aerodynamic performance to Reynolds number was confirmed for several flap configurations throughout the test, with results similar to those shown in Figures 4-9. 


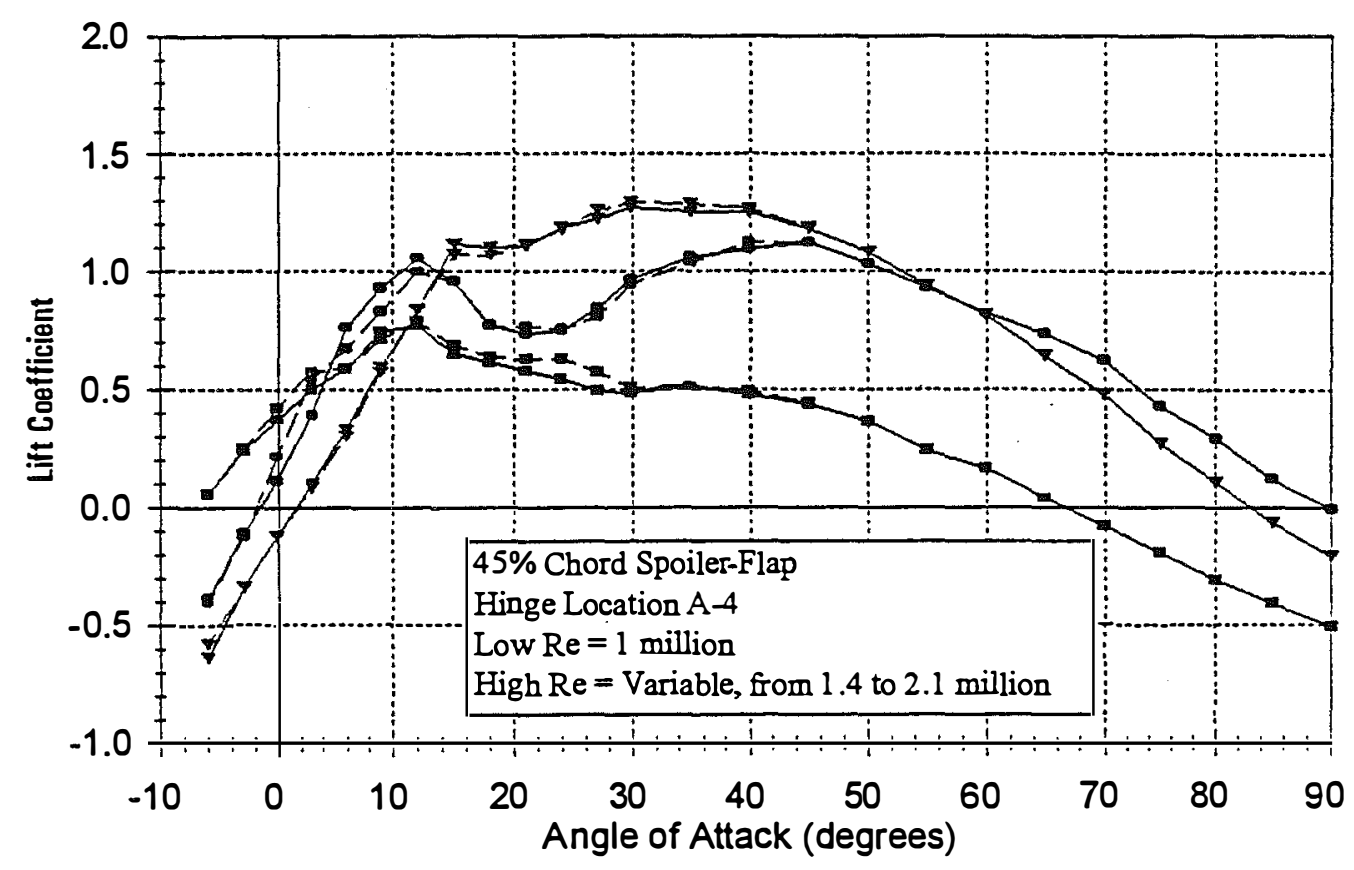

$\rightarrow$ Delta $=0$ (Low Re) $\rightarrow-$ Delta $=0$ (High Re) $\rightarrow$ Delta $=30$ (Low Re)

$-*-$ Delta $=30($ High Re $) \rightarrow$ Delta $=60($ Low Re $) \rightarrow-$ Delta $=60($ High Re $)$

Figure 4-9a. Lift Data, Reynolds Number Effect on Spoiler-Flap

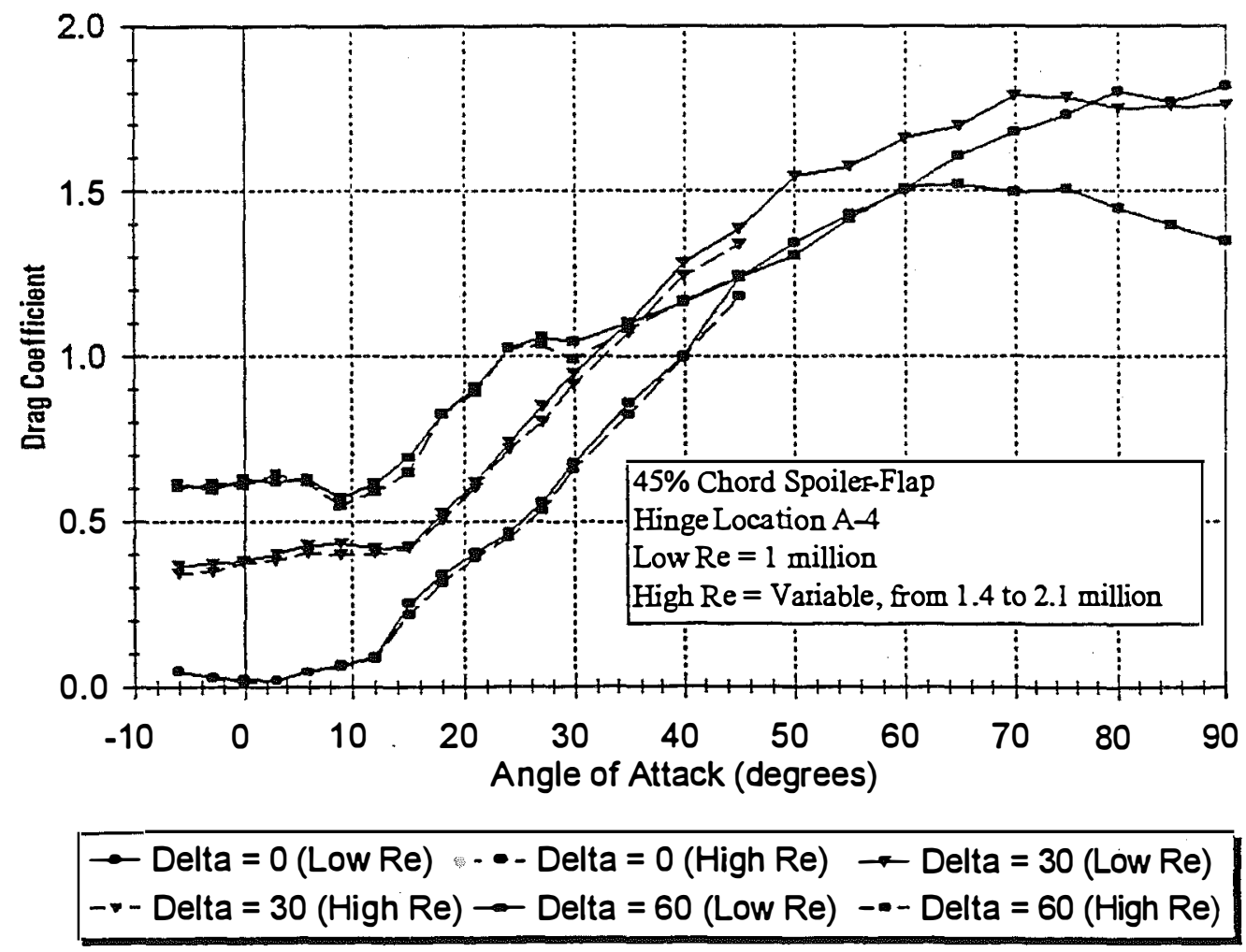

Figure 4-9b. Drag Data, Reynolds Number Effect on Spoiler-Flap 


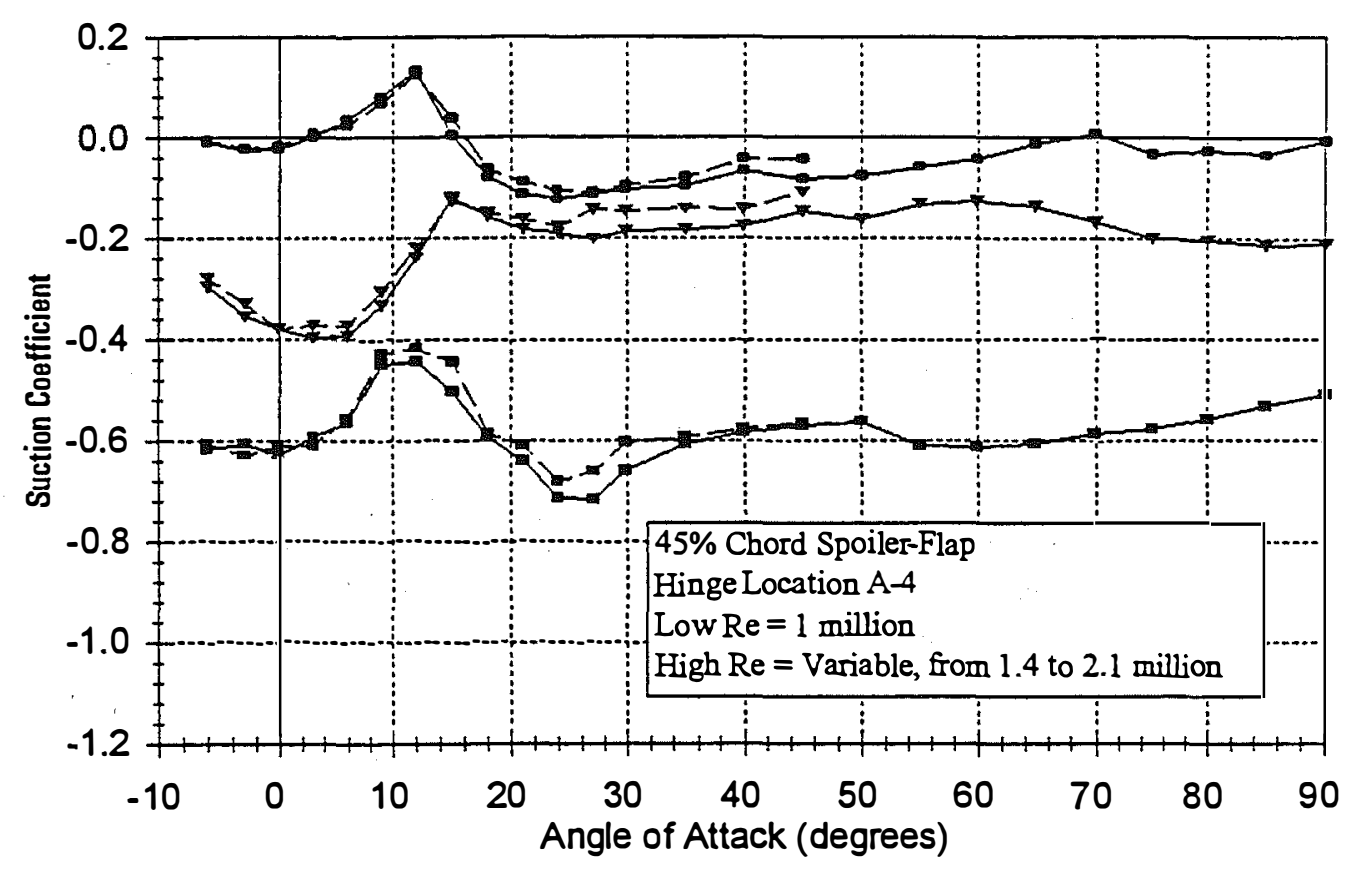

$$
\begin{aligned}
& \rightarrow \text { Delta }=0(\text { Low Re }) \rightarrow-\text { Delta }=0(\text { High Re }) \rightarrow \text { Delta }=30(\text { Low Re }) \\
& \rightarrow-\text { Delta }=30(\text { High Re }) \rightarrow \text { Delta }=60(\text { Low Re }) \rightarrow-\text { Delta }=60(\text { High Re })
\end{aligned}
$$

Figure 4-9c. Suction Data, Reynolds Number Effect on Spoiler-Flap

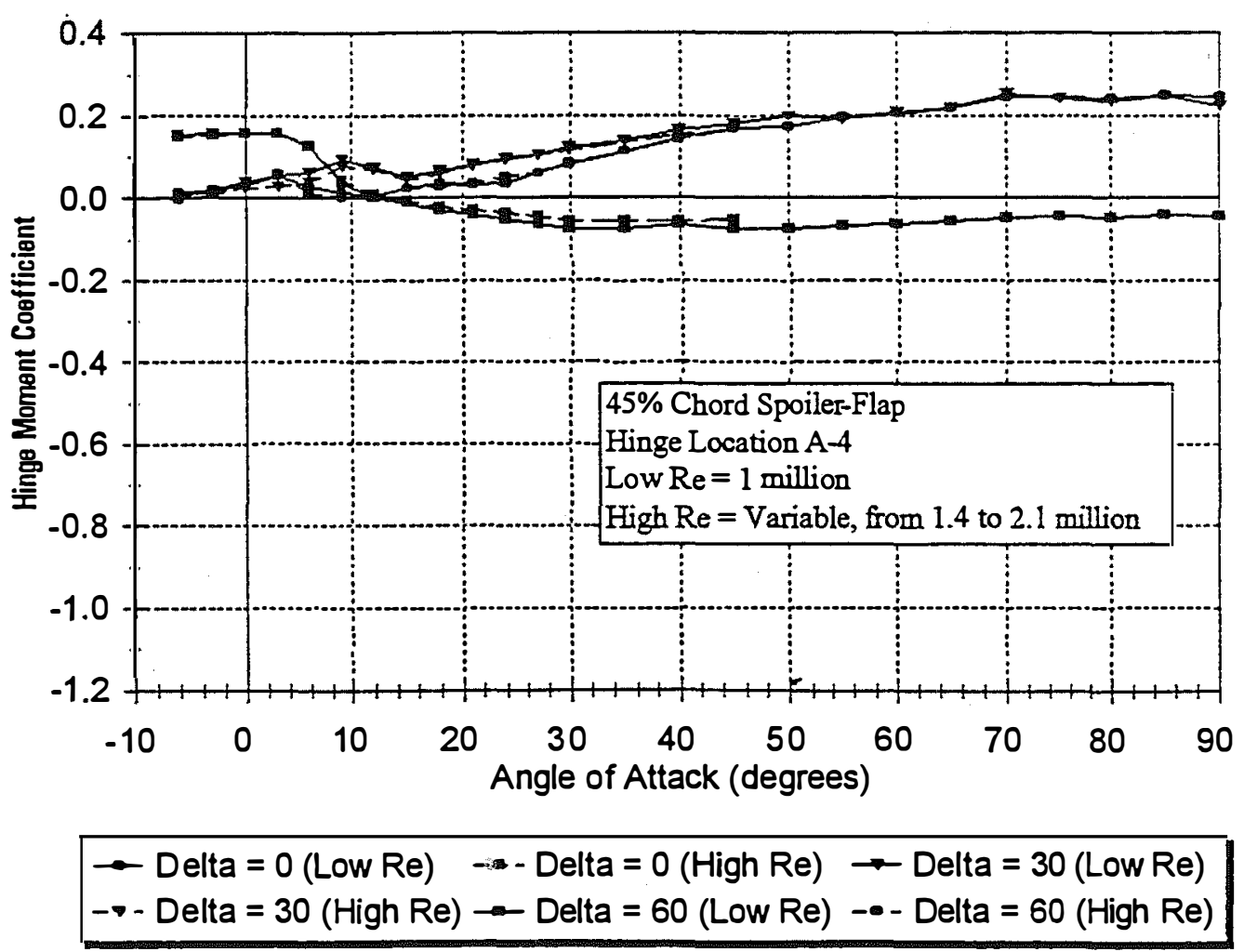

Figure 4-9d. Hinge-Moment Data, Reynolds Number Effect on Spoiler-Flap 


\subsubsection{Partial-Span Effects}

The motivation for considering partial-span effects was to determine whether the two-dimensional flap data could be used directly to predict the stopping power of actual (finite aspect ratio) devices, or whether a three-dimensional "knock-down" should be applied. As an example, the drag coefficient of an infinite flat plate is approximately 2.0 , but drops rapidly to a value of 1.2 for plates of finite aspect ratio [10]. For flat-plate drag coefficients, this would suggest a finite aspect ratio knock-down of $40 \%$ from the two-dimensional value. Although the flap configurations which were tested are more than simple drag devices, it is still important to consider the impact of aspect ratio on their aerodynamic effectiveness.

The baseline model for the wind tunnel tests had flaps that were of infinite aspect ratio, extending the entire airfoil span from floor to ceiling. Although the importance of aspect ratio effects was recognized, the design and construction of a segmented model (capable of partial-span flap deployment) was beyond the resources of this project.

As a compromise, inexpensive bolt-on plates were designed to approximate the effect of aspect ratio on flap performance. Figure 4-10 shows the dimensions and placement of the plates on the WSU model. The plates were constructed in three lengths: 1/3-span, 1/2-span, and full-span. The partial-span plates were installed such that the center of their span was at or near the center of the airfoil span.

The plates could be bolted on in a modular fashion to model various devices. For example, the installation of plate $\# 2$ would model a split-flap deployed to $\delta=45^{\circ}$. In terms of projection into the free-stream, installation of both plates \#1 and \#3 would closely resemble a spoiler-flap deployed to $90^{\circ}$. Unfortunately this arrangement does not allow for the venting of flow that would occur for an actual deployed spoiler-flap, which is considered to be a key feature of the device aerodynamics. In all cases, the partial-span plates were considered to accurately model flaps only at low airfoil angles of attack, prior to significant impingement of the free-steam flow on the trailing edge of the rear model element.

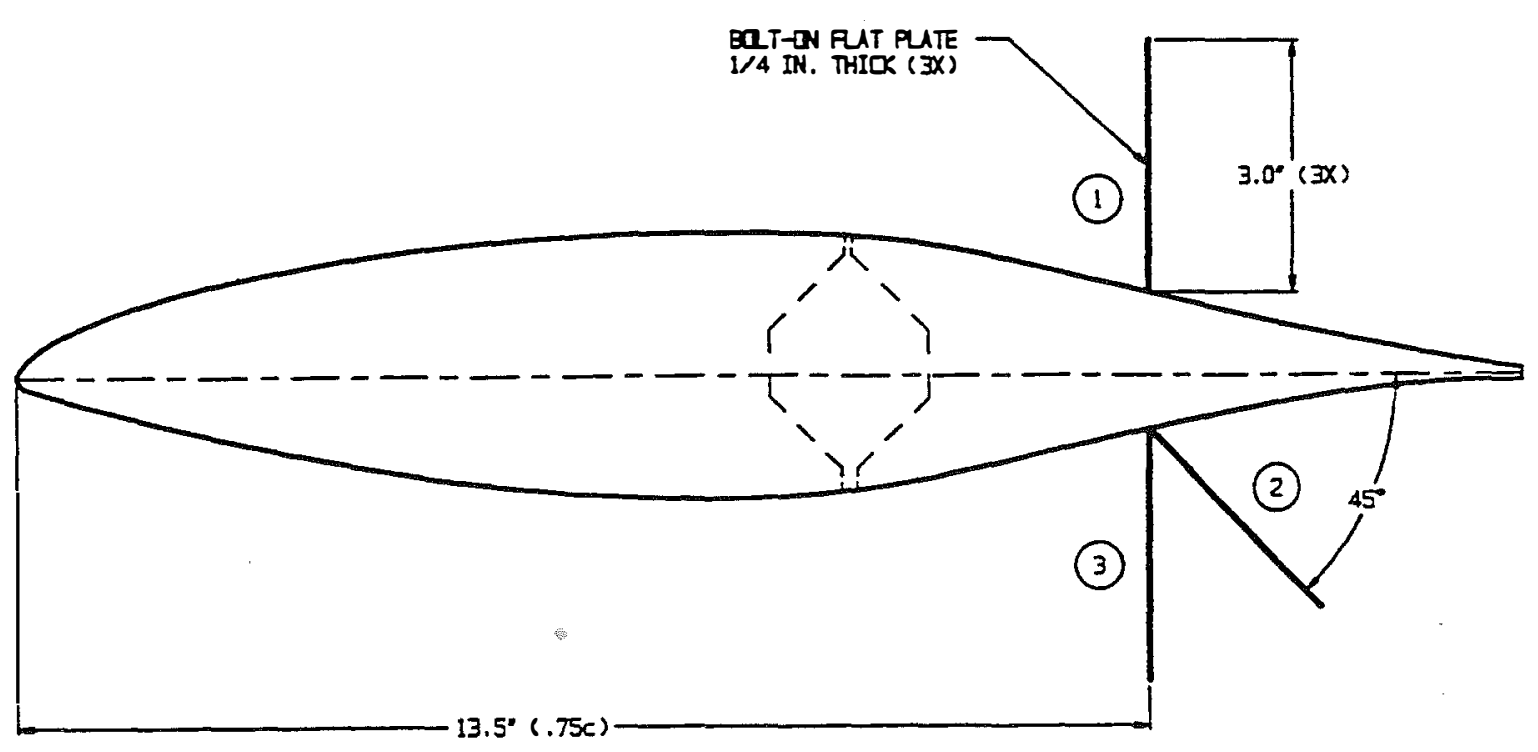

Figure 4-10. Sectional View of Model with Partial-Span Plates 
During the wind tunnel test, the total resultant aerodynamic forces on the partial-span models were normalized with respect to the full-model dimensions. Therefore, the coefficients for partial-span configurations require additional analysis to be correctly interpreted. The total force on a model with a partial-span flap could be considered as a superposition of the aerodynamic forces on the modified and unmodified portions of the airfoil. The wind tunnel coefficients shown in this report were re-reduced assuming such a linear superposition of forces, although this is an admitted oversimplification of the flow around the partial-span model.

Figure 4-11 shows an example of suction coefficient behavior from the partial-span tests. The data are given in knock-down form, expressed as a percentage loss of effectiveness due to finite aspect ratio:

$\Delta \mathrm{C}_{\mathrm{S}}$ knock - down $=100 * \frac{\Delta \mathrm{C}_{\mathrm{S} \text {, full-span flap }}-\Delta \mathrm{C}_{\mathrm{S} \text {, partial-span flap }}}{\Delta \mathrm{C}_{\mathrm{S}, \text { full-span flap }}}$.

For $\alpha<12^{\circ}$, the data show a fairly consistent knock-down of $20 \%$ to $30 \%$, which is consistent with flat-plate drag effects. The data become erratic at post-stall angles of attack, which is understandable as the assumptions of linearity become less valid and airflow begins impinging on the trailing-edge of the model. The legend in Figure 4-11 shows the flap configurations tested, with $90 / 90$ indicating $90^{\circ}$ plates on both the upper and lower surfaces (Plates \#1 and \#3 shown in Figure 4-10) and 90/45 indicating a $90^{\circ}$ plate on the upper surface and a $45^{\circ}$ plate on the lower surface (Plates $\# 1$ and $\# 2$ ).

Although a strong dependence on aspect ratio was found, the application of these data to spoiler-flap and flip-tip design is uncertain. The partial-span models did not include flow venting, which is considered to be a key feature of spoiler-flap aerodynamics. The work of References 5 and 6 is intended to assess the full three-dimensional effects on both ailerons and spoiler-flaps, but the results of this-work have not yet been published.

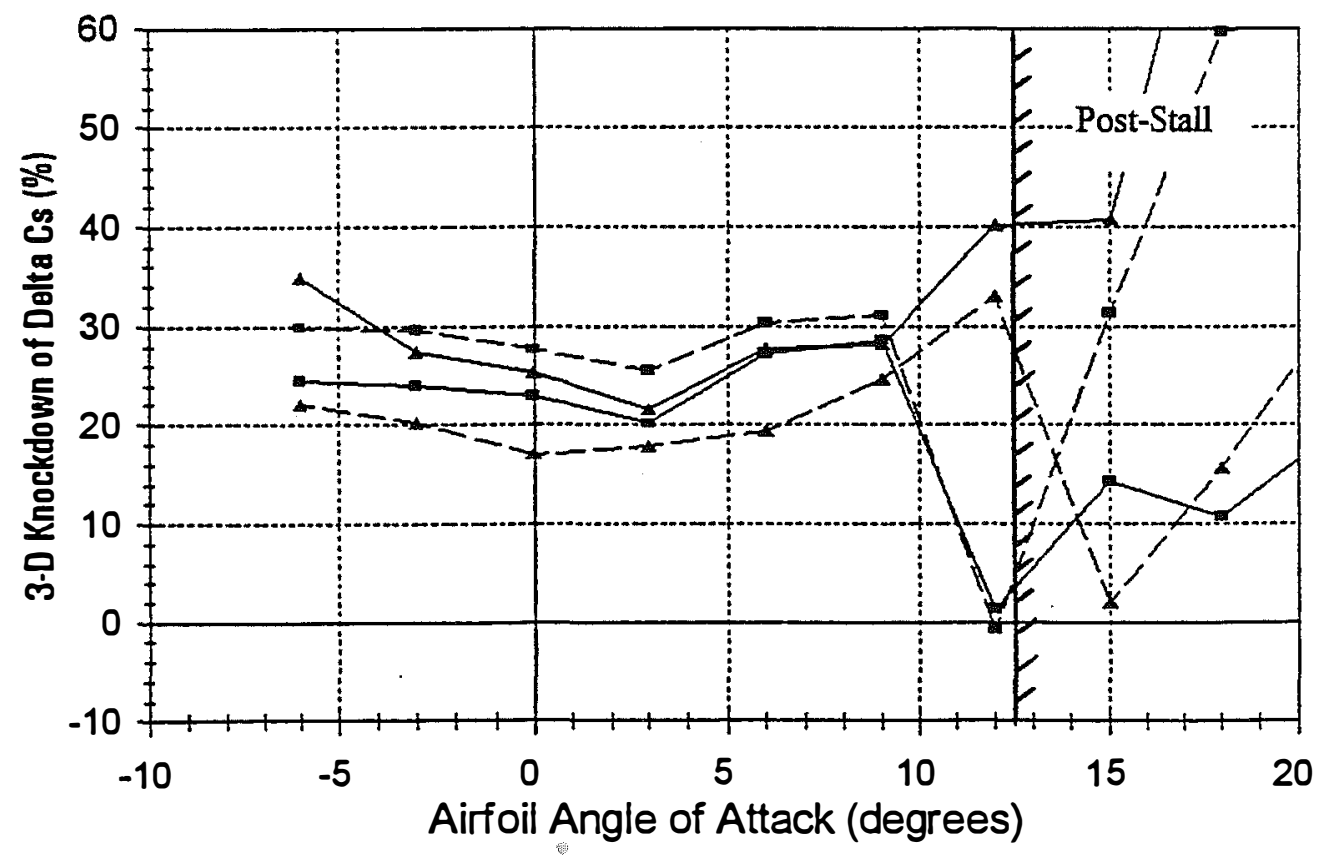

- 1/2 span 90/90 - - 1/3 Span 90/90 $\leftarrow$ 1/2 Span 90/45 -1 - 1/3 Span 90/45

Figure 4-11. Effect of Finite Aspect Ratio on Flap Braking Effectiveness 


\section{Sizing and Deployment Studies}

\subsection{AWT-26/27 Braking Requirements}

The braking requirements for AWT-26/27 turbines are dependent on the magnitude of the power that is captured by the rotor under various operating conditions. The rotor power can be related to torque at the low-speed shaft by

$\mathrm{P}_{\text {Rotor }}=\mathrm{M}_{\mathrm{LSS}} \cdot \Omega$

where $M_{L s s}$ is the low-speed shaft torque and $\Omega$ is the rotor speed expressed in radians per second. Direct measurement of low-speed shaft torque is possible, but can be difficult. An alternative method of determining rotor torque is by measuring electrical power output, then adjusting for drivetrain losses. This second method has been used to generate Figure 5-1. The original data were in the form of a generator power curve measured from the AWT-26 prototype turbine, P1. Drivetrain efficiencies were used along with Equations 2-6 and 2-7 to establish a rotor $C_{P}$-TSR curve for the AWT-26 at an operating speed of $57 \mathrm{rpm}$. With the assumption that the $\mathrm{C}_{\mathrm{P}}$-TSR curve remained unchanged, the rotor power curves of Figure 5-1 were developed for wind speeds up to $30 \mathrm{~m} / \mathrm{s}(67 \mathrm{mph})$ and rotor speeds between 50 and $75 \mathrm{rpm}$. Each curve has been labeled with the maximum low-speed shaft torque.

This figure shows the relationship between rotor speed and braking requirements. The AWT-26/27 rotors were designed for normal operating speeds between 50 and $60 \mathrm{rpm}$. The $75 \mathrm{rpm}$ curve therefore

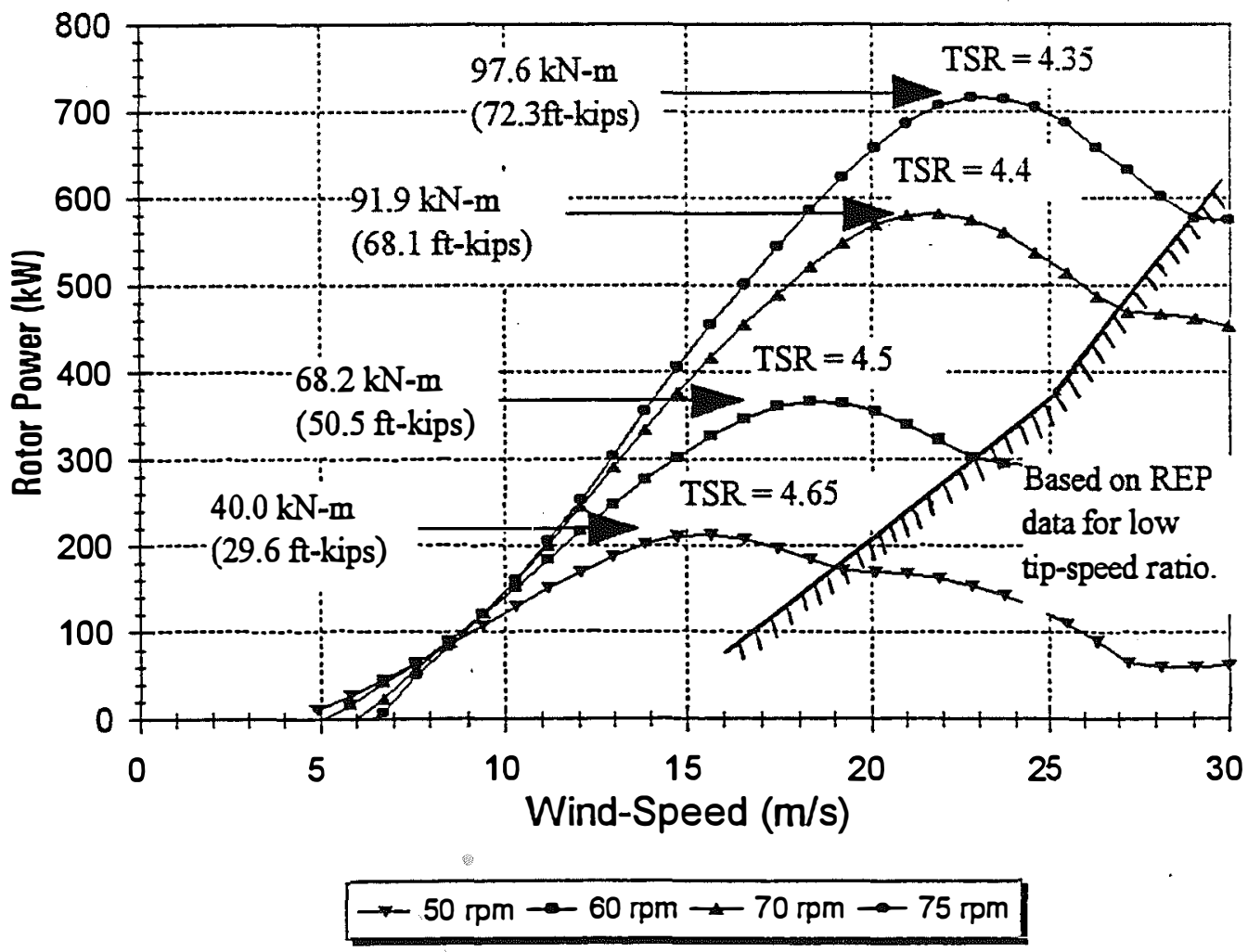

Figure 5-1. AWT-26 Rotor Power at Varying Rotational Speeds 
represents a $25 \%$ overspeed beyond the maximum normal operation of the AWT-26. The curves show a doubling of maximum rotor power due to this overspeed condition, and a $43 \%$ increase in the peak low-speed shaft torque. In the absence of external load (i.e., freewheeling rotor), these values represent the amount of power that must be extracted from the rotor by either mechanical or aerodynamic brakes in order to prevent the rotor from further acceleration.

The tip-speed ratio at maximum rotor power is noted on each of the power curves of Figure 5-1. At wind speeds above peak power (decreasing tip-speed ratios), the power levels are strictly decreasing for all wind speeds shown. It should be noted that the power curve for the P1 prototype included only measured tip speeds of 3.5 and greater, which are shown as data to the left of the hashed lines on Figure 5-1. The data for tip-speed ratios below 3.5 were obtained during the AWT ESI-80 Performance and Reliability Enhancement Program (REP) [11]. The REP data were obtained with AWT-26 blades retrofitted to an ESI-80 turbine, and the rotor pitched to a peak generator power of 35 $\mathrm{kW}$ at a rotor speed of $30 \mathrm{rpm}$. Therefore, the REP configuration was somewhat different than the current AWT-26 design, and the low TSR data presented here are not considered of high enough confidence for aerodynamic brake design.

All specific sizing and performance calculations in the following sections have been made for the AWT-26 turbine. Although Figure 5-1 presents data in terms of rotor power and shaft torque, the majority of the work in this report will be expressed in coefficient form. This is done because $\mathrm{C}_{\mathrm{P}}$-TSR curves are able to represent the wide range of wind and rotor speeds over which the aerodynamic brakes must be effective. It will be shown that the most critical point of operation for aerodynamic brakes occurs at low tip-speed ratios. Due to the need for low TSR data, the baseline AWT-26 $\mathrm{C}_{\mathrm{P}}$-TSR curve has been generated by calculations using the PROP93 analytic code [12]. Figure 5-2

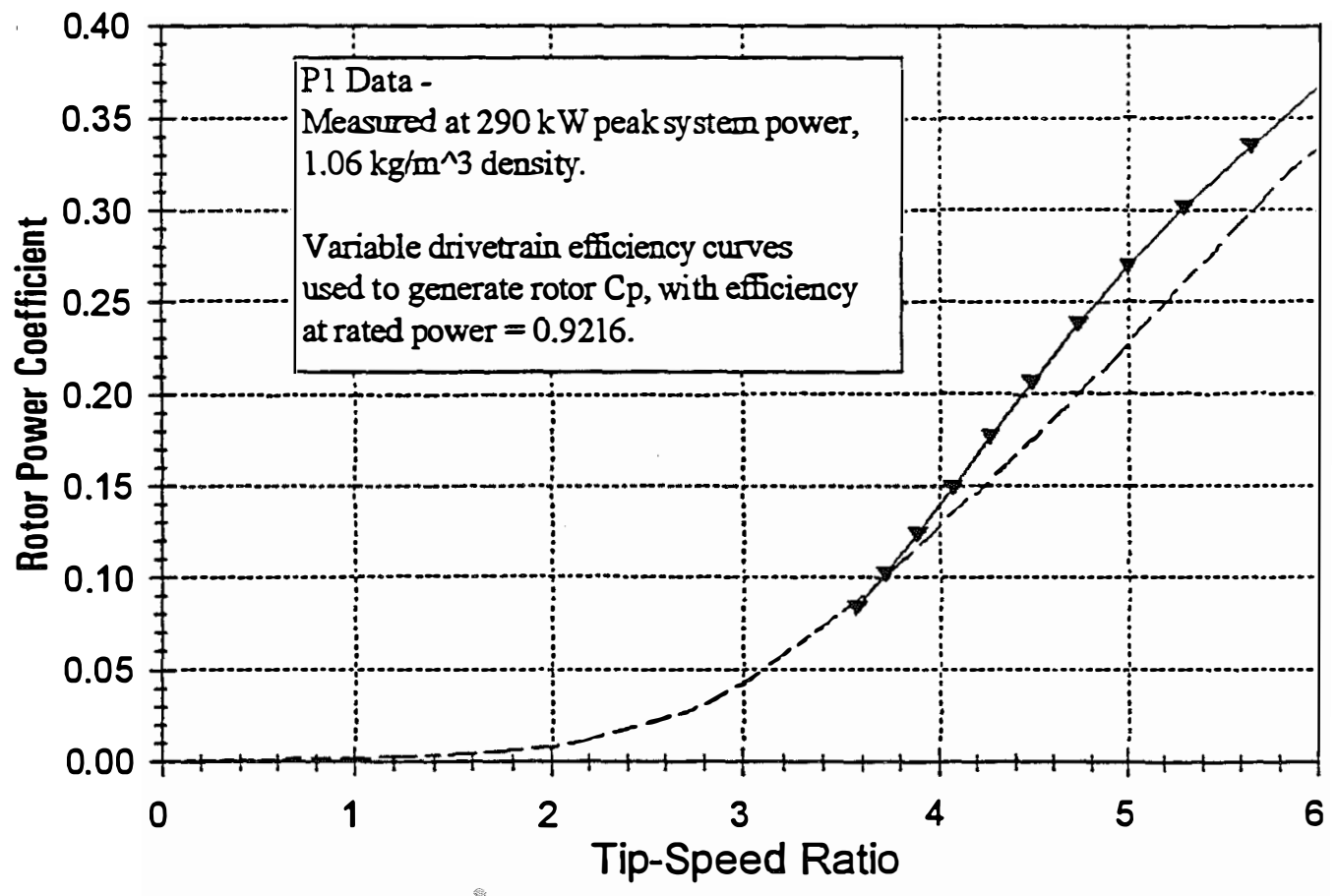

-- PROP Calculations $\rightarrow$ Measured P1

Figure 5-2. Rotor Power Coefficients for AWT-26 at Low TSR 
shows a comparison of PROP93 versus measured P1 power coefficients at low tip-speed ratios. The curves show very close agreement for tip-speed ratios between 3.5 and 4.0, which represent the AWT-26 at post-stall wind speeds near $22 \mathrm{~m} / \mathrm{s}(50 \mathrm{mph})$. Peak power for normal AWT-26 operation occurs at a TSR of about 4.5, where the PROP93 values for $C_{P}$ are somewhat low. The sizing of aerodynamic brakes for the AWT-26 is strongly dependent on the power coefficients near a tip-speed ratio of 3.0, where the PROP93 calculations show $C_{P} \approx 0.04$. For this rotor, low tip-speed ratios correspond to high blade angles of attack $\left(\alpha_{\text {tip }} \geq 18^{\circ}\right)$, and the accuracy of the PROP93 calculations is uncertain. However, in the absence of a better method, the analytic data shown in Figure 5-2 will be used as a baseline for the following aerodynamic braking calculations.

\subsection{Sizing of Candidate Devices}

AWT engineers have specified design load cases for the aerodynamic braking system [13] that are consistent with international wind turbine design standards $[14,15]$. The AWT specifications have been developed for a Class II wind site as defined by Reference 14. This report will only address the load cases that have been found to dominate the sizing and deployment characteristics of the aerodynamic brakes.

The AWT-26/27 turbines are designed for a range of operational speeds from 50 to $60 \mathrm{rpm}$. Per the requirements of Reference 15, the braking system design will assume that a maximum rotor speed of $25 \%$ above synchronous may occur during an overspeed condition. The aerodynamic braking system will therefore be required to prevent rotor speeds in excess of $75 \mathrm{rpm}$ for all design load cases.

Several load cases include turbine faults that may lead to loss of load and mechanical brakes. In these cases the rotor is freewheeling, and the aerodynamic braking system must prevent rotor speeds from exceeding $75 \mathrm{rpm}$. In accordance with Reference 14, the freewheeling condition was assumed to occur during a 1-year extreme operating gust described by:

$\mathrm{V}=\mathrm{V}_{\text {hub }}+0.5 \cdot \mathrm{V}_{\text {gust }}[1-\cos (2 \pi \mathrm{t} / \mathrm{T})]$

where $T=12 \mathrm{~s}$. For a Class II wind site, the normal turbulence model of Reference 14 defines the standard deviation of wind speed as $\sigma=0.153 \cdot \mathrm{V}_{\text {hub }}+0.277(\mathrm{~m} / \mathrm{s})$, and a 1 -year gust as $\mathrm{V}_{\text {gust }, 1}=3.75 \cdot \sigma$. The highest value of $\mathrm{V}_{\text {gust, } 1}$ would be obtained for a turbine operating near cut-out wind speeds, nominally $22.3 \mathrm{~m} / \mathrm{s}(50 \mathrm{mph})$ for the AWT-26/27 turbines. Thus, for the AWT-26/27 turbines, the 1-year extreme operating gust would result in a maximum wind speed of $36.2 \mathrm{~m} / \mathrm{s}(81.0$ $\mathrm{mph}$ ), with the gust event occurring over a 12-second interval.

It was found that the above case dominated the sizing of candidate aerodynamic brakes. A device sized to restrain freewheeling rotor speeds to $\Omega_{\max } \leq 75 \mathrm{rpm}$ during a 1-year extreme operating gust was generally suitable for all other design cases (assuming adequate deployment). The sizing and deployment studies presented in the following sections are all based on the 1-year extreme operating gust.

Equation 2-8 can be used to determine the maximum freewheeling tip-speed ratio allowed for given rotor and wind-speeds. Substituting values of $\Omega_{\max }=7.85 \mathrm{rads} / \mathrm{s}(75 \mathrm{rpm}), \mathrm{V}_{0}=36.2 \mathrm{~m} / \mathrm{s}$, and $\mathrm{R}=13.1 \mathrm{~m}(43 \mathrm{ft})$ yields a value of $\mathrm{TSR}_{\max }=2.8$ for the AWT-26 turbine. Equilibrium tip-speed ratios greater than 2.8 would allow the freewheeling rotor to exceed $75 \mathrm{rpm}$ during a 1-year gust. This illustrates the importance of low tip-speed ratios for aerodynamic braking of the AWT-26/27 turbines. 
A review of the wind-tunnel data for spoiler-flap devices shows that the $\Delta \mathrm{C}_{\mathrm{s}}$ supplied by the devices is dependent on the deployment angle. In particular, there is a significant increase in device effectiveness between the angles of $60^{\circ}<\delta<75^{\circ}$, with a much smaller performance increment for angles greater than $75^{\circ}$. The sizing of aerodynamic brakes is therefore dependent on the specifics of the design, including deployment rates and equilibrium deployment angles. The trade-off between device size and deployment characteristics will be addressed in Section 5.3.1. The present sizing analysis assumes all devices are fully deployed to $\delta=90^{\circ}$.

Results from the sizing studies will be presented in coefficient form. Equation 2-1 has been used with the wind-tunnel data to calculate $\Delta \mathrm{C}_{\mathrm{S}}$ for each device geometry and deployment angle $\left(\delta=90^{\circ}\right.$ for device sizing). As discussed below, an adjustment has been applied to the two-dimensional data to account for loss of effectiveness due to finite aspect ratio. Equations 3-8 and 3-9 have then been used to determine the rotor $\Delta C_{P}$ for each device geometry. This process included PROP93 calculations to account for the effect of rotor interference on turbine-blade angles of attack.

Figures 5-3 show results from this method, where the TSR range has been truncated to emphasize the results at low tip-speed ratios. Finite aspect ratio knock-downs of $20 \%$ have been applied to the flap sections (spoiler and plain) and 30\% for the tip region of the flip-tip. Note that the $30 \%$ knock-down for the tip region was determined as the factor required to adjust the WSU wind-tunnel data to obtain the finite flat-plate result of $C_{D_{\max }}=1.2$. As the actual finite aspect ratio adjustments for these devices are unknown, the data on Figures 5-3 should not be considered absolute, but are rather meant to illustrate the sizing trends of the devices. Several different knock-down factors were used in the course of this work. Each analysis shown in this report will be labeled with the knock-down factors that were used in converting the two-dimensional wind tunnel data.

Figure 5-3a shows sizing results for the flip-tip, where adequate braking is obtained for devices with a total span as low as $10 \%$. The figure also demonstrates that the flip-tip remains effective for a variety of tip/flap area ratios. The correct size-weighting of the tip and flap regions will be of greatest importance when determining deployment characteristics.

With a $20 \%$ knock-down, Figure 5-3b shows the $15 \%$ span spoiler-flap to be undersized, with an equilibrium tip-speed ratio of 3.7. Although the spoiler with $17.5 \%$ span maintains negative values of $\mathrm{C}_{\mathrm{P}}$ for all TSR $>1.0$, inspection of the curves near TSR $=3.0$ shows that this device is marginally sufficient.

Similar sizing studies were performed for the $38 \%$ chord spoiler-flap. The general result was not surprising; $38 \%$ chord spoiler-flaps require a longer span (by about $5 \% \mathrm{R}$ ) to have stopping power equivalent to the $45 \%$ chord device. The $38 \%$ flap would remove less chordwise structure from the existing blade, but the cut-line would occur over a larger span. Additionally, hinge design for the $38 \%$ chord spoiler would be more challenging, as the maximum device cross section would be smaller. After consideration of the structural and mechanical issues involved in the sizing, AWT engineers concluded that the $45 \%$ chord device was preferable for implementation on the AWT-26/27 blades. Further analysis and discussion of spoiler-flaps will, therefore, be restricted to $45 \%$ chord devices.

Note that the sizing results shown are for spoiler-flaps that extend to the blade tip, which is the furthest outboard placement possible. Equation 3-8 shows a cubic dependency on radial position of the device that favors outboard placement. This implies a significant penalty for moving the device inboard. 


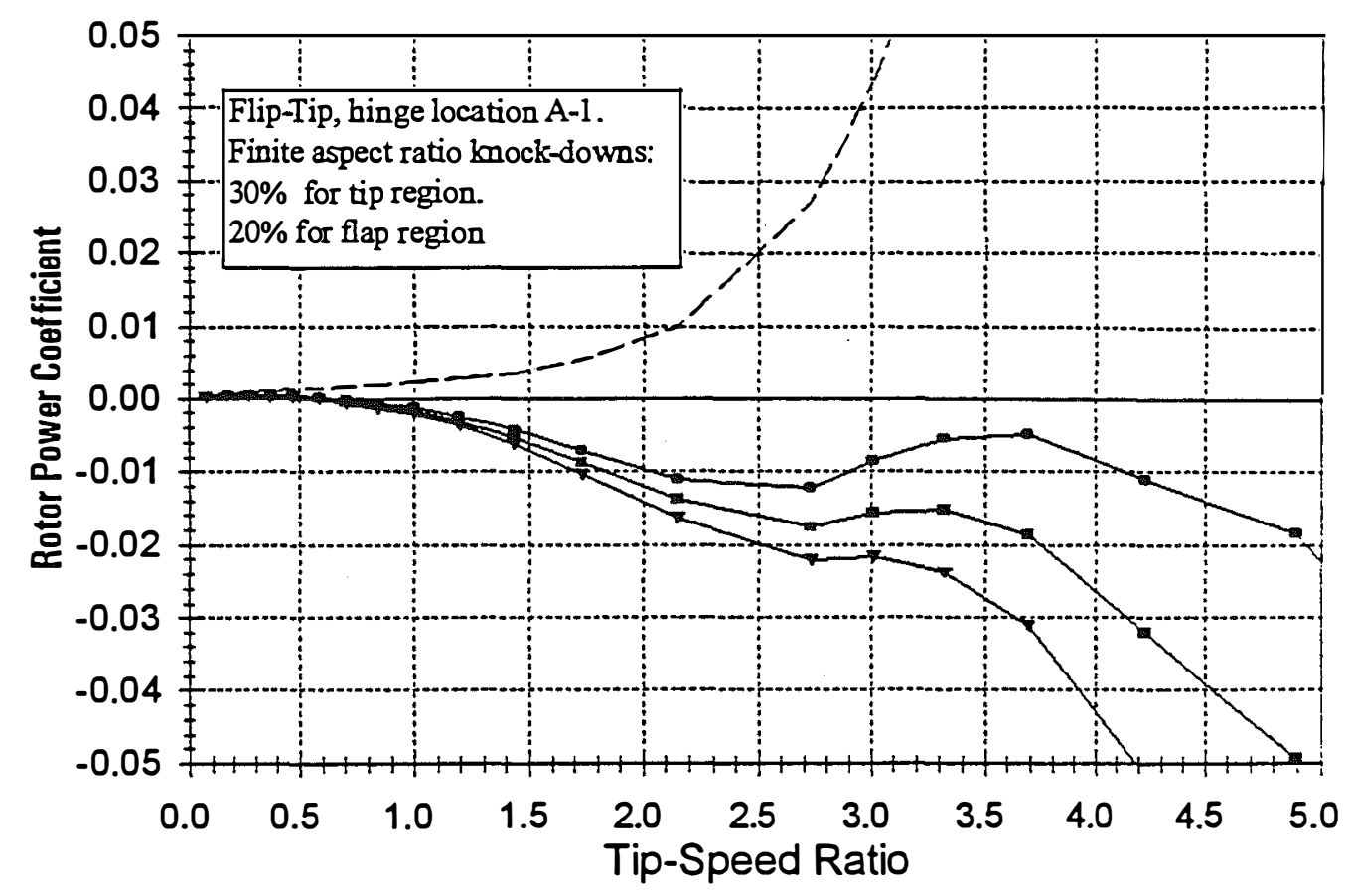

-- No Flip Tip $\rightarrow 7.5 \%$ tip/ $5 \%$ flap $\rightarrow 6 \%$ tip/ $4 \%$ flap $\rightarrow 7 \%$ tip/ $4 \%$ flap

Figure 5-3a. Sizing of Flip-Tip for AWT-26 Aerodynamic Braking

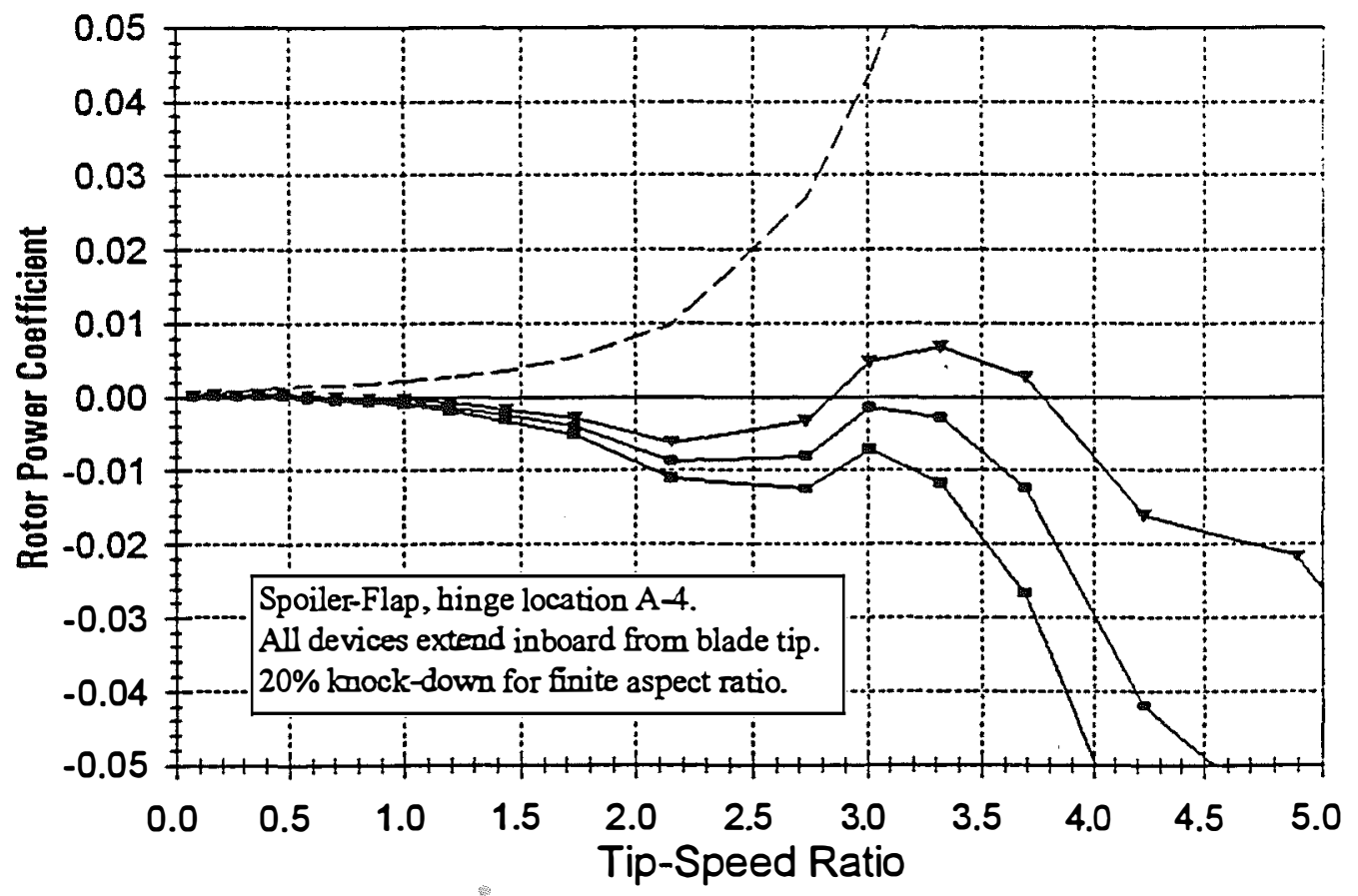

-- No Spoiler $\rightarrow 15 \%$ Span $\rightarrow 17.5 \%$ Span $\rightarrow 20 \%$ Span

Figure 5-3b. Sizing of Spoiler-Flap for AWT-26 Aerodynamic Braking 
However, $\Delta \mathrm{C}_{\mathrm{P}}$ also varies linearly with the area of the modified blade section, and the overall effectiveness will scale as the product $(\mathrm{r} / \mathrm{R})^{3} \cdot \mathrm{A}_{\text {section}}$. For turbines with significant blade taper at the tip, the increase in modified blade area can outweigh the loss in $(r / R)^{3}$. This is demonstrated for the AWT-26/27 turbine in Table 5-1, which shows overall effectiveness increasing slightly and then falling off slowly as the device is moved inboard.

Table 5-1. Variation of Spoiler-Flap Effectiveness with Spanwise Location

\begin{tabular}{ccccc}
\hline $\begin{array}{c}\text { Device } \\
\text { Center }(\mathrm{r} / \mathrm{R})\end{array}$ & $\begin{array}{c}\text { Blade Chord at } \\
\text { Device Center }(\mathrm{cm})\end{array}$ & $\begin{array}{c}\text { Blade Thickness at } \\
\text { Device Center }(\mathrm{cm})\end{array}$ & $\begin{array}{c}\text { Blade Area at } \\
\text { Device }\left(\mathrm{m}^{2}\right)\end{array}$ & $\begin{array}{c}(\mathrm{r} / \mathrm{R})^{3} \cdot \mathrm{A}_{\text {section }} \\
\left(\mathrm{m}^{2}\right)\end{array}$ \\
\hline 0.925 & 52.7 & 9.22 & 1.007 & 0.797 \\
0.900 & 57.0 & 9.98 & 1.096 & 0.799 \\
0.875 & 61.3 & 10.73 & 1.184 & 0.793 \\
0.850 & 65.5 & 11.46 & 1.250 & 0.767 \\
0.825 & 68.9 & 12.06 & 1.333 & 0.748 \\
\hline
\end{tabular}

Note: Assumes $15 \%$ span spoiler-flap on AWT-26 blade.

\subsection{Aerodynamic Deployment Calculations}

The AWT-26/27 tip-vane deployment is governed by a combination of aerodynamic and centripetal forces, with the centripetal effects dominating the hinge moments at small deployment angles, and aerodynamics dominating at large angles. In principle, either the spoiler-flap or flip-tip configuration could be aerodynamically deployed in a manner very similar to the AWT-26/27 tip vanes. However, the orientation of the spoiler-flap and flip-tip configurations tends to diminish centripetal moments about the device hinge lines. Section 5.3 will focus on deployment by a combination of aerodynamics and passive mechanical forces (springs and dampers), neglecting centripetal effects. Sections 5.4 and 5.5 will include details of how centripetal forces may be used to assist in either active or passive mechanical deployment methods. With proper design, of course, centripetal forces could be used to assist aerodynamically deployed devices.

Analysis of aerodynamic deployment for the spoiler-flap and flip-tip configurations begins with the general equations of motion:

$\sum \mathrm{M}_{\mathrm{Rotor}}=\mathrm{I}_{\mathrm{Rotor}} \cdot \dot{\Omega}+\mathrm{C}_{1} \cdot \Omega+\mathrm{C}_{2}$

where:

$\mathbf{M}_{\text {Rotor }} \equiv$ all moments contributing to rotation about the low-speed shaft

$\mathrm{I}_{\text {Rotor }} \equiv$ rotational moment of inertia of the rotor system and drivetrain about the low-speed shaft

$\dot{\Omega} \quad \equiv$ rate of change of rotor speed

$\mathrm{C}_{1} \cdot \Omega \quad \equiv$ accounts for variable-speed component of generator and gearbox load

$\mathrm{C}_{2} \equiv$ accounts for constant drivetrain load or losses

and,

$\sum \mathrm{M}_{\text {Hinge-Line }}=\mathrm{I}_{\text {Flap }} \cdot \ddot{\delta}+\mathrm{C}_{3} \cdot \dot{\delta}+\mathrm{C}_{4} \cdot \delta$ 
where:

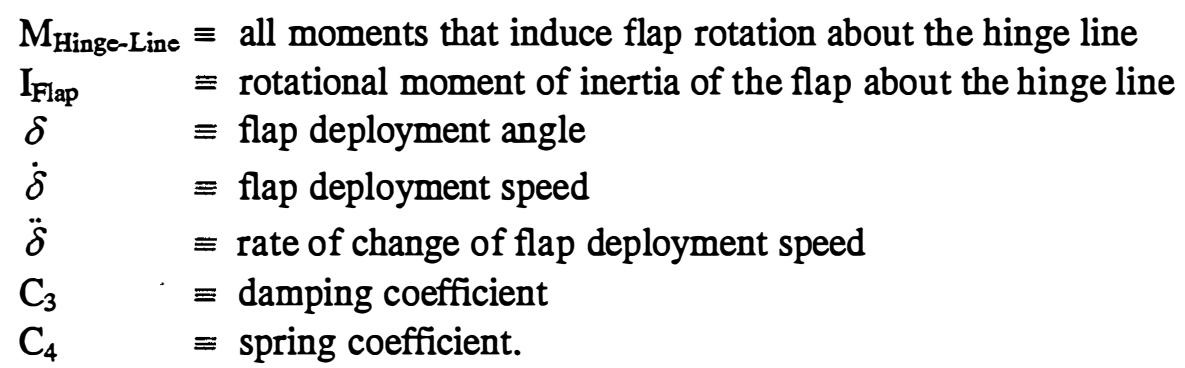

Equations 5-3 and 5-4 are second-order, nonlinear differential equations that are fully coupled. Any change of wind or rotor speed would change the aerodynamic condition of both the rotor and the flaps. Conversely, a change in flap deployment would change the aerodynamic torque (power) supplied to the rotor. Equation 5-3 is written in a turbine-fixed frame of reference while Equation 5-4 is in a rotating blade-fixed frame. These equations can be solved simultaneously so long as the appropriate auxiliary equations are introduced that relate the two reference frames. The equations can be reduced to a system of first-order differential equations in a form suitable for Runge-Kutta integration by the introduction of state variables.

let:

$\mathrm{y}_{1}=\Omega$

$\mathrm{y}_{2}=\delta$

$\mathrm{y}_{3}=\dot{\delta}$

then:

$$
\begin{aligned}
& \dot{y}_{1}=\left[I_{\text {Rotor }}\right]^{-1} \cdot\left(\sum M_{\text {rotor }}-C_{1} \cdot y_{1}-C_{2}\right) \\
& \dot{y}_{2}=y_{3} \\
& \dot{y}_{3}=\left[I_{\text {Flap }}\right]^{-1} \cdot\left(\sum M_{\text {Hinge- line }}-C_{3} \cdot y_{3}-C_{4} \cdot y_{2}\right)
\end{aligned}
$$

With initial conditions for $\Omega, \delta$, and $\dot{\delta}$, a time history of the aerodynamic brake deployment can be obtained through integration of Equations 5-5. This requires that the right-hand side of these equations be evaluated at each time step. Several of the inputs, such as $I_{\text {Rotor }}, I_{F l a p}, C_{1}, C_{2}, C_{3}$, and $C_{4}$, are constant or are available from mechanical considerations. The remaining terms on the right-hand side of Equations 5-5 involve externally applied moments.

The low-speed shaft moments, $\Sigma \mathrm{M}_{\text {Rotor }}$ can be evaluated in terms of $\mathrm{C}_{\mathrm{P}}$ versus TSR curves for the rotor with and without deployed aerodynamic brakes (refer to Figure 2-2). For a given flap deflection, the $C_{P}$-TSR curves can be used along with wind speed and air density to find the rotor power, which can be used to solve for the rotor's aerodynamic contribution to the low-speed shaft torque. Any mechanical braking can be subtracted directly from the right-hand side of Equation 5-5a. 
The $\Sigma \mathrm{M}_{\text {Hinge-moment }}$ term is made up of two major contributors: aerodynamic and centripetal. The aerodynamic hinge moments are functions of the type and size of device, hinge location, air density, wind speed, $\Omega, \delta$, and $\alpha$. Centripetal moments are functions of flap mass, location of device center of gravity, hinge-line location and orientation, blade coning angle, $\delta$, and $\Omega$. As discussed above, the present analysis neglects centripetal hinge moments.

A FORTRAN code was written to perform the integration of Equations 5-5. The code allows fast simulations of aerodynamic brake deployments, with user-specified inputs of initial conditions, wind speed, mechanical braking applied, and mechanical characteristics of the devices. Quatro-Pro workbooks were designed to convert wind-tunnel data into families of $C_{P}-T S R$ and $C_{h}-\alpha$ curves, which represent the geometry, size, and placement of each candidate device. Graphical examples of these curves will be shown in the following sections. Tabular forms of the curves were read by the deployment code as look-up tables, allowing the calculations to update the aerodynamic performance of both the device and the rotor at each time step.

\subsubsection{Spoiler-Flap}

During the course of this work several iterations of spoiler-flap sizing, deployment simulation, and preliminary mechanical design were performed. This section discusses some of the general trends and design issues that were identified as a result of these iterations. Some of these results may be specific to the design of spoiler-flaps for use on the AWT-26/27 rotors. Nonetheless, the discussion should be instructive towards the use of spoiler-flaps with other turbine designs.

This work focused on an aerodynamic deployment strategy that was very similar to the current AWT26/27 tip vane:

1. The aerodynamic brake assists with all normal and emergency stopping sequences, and provides overspeed protection for the case of a freewheeling rotor.

2. The spoiler-flap is hinged far enough aft so that it would have a strong aerodynamic tendency to deploy (positive aerodynamic hinge moments).

3. During normal turbine operation the flap is held closed by a combination of electromagnetic force and spring pre-load.

4. De-energizing the electromagnet allows the device to open aerodynamically, with the deployment rate controlled by a combination of aerodynamic, spring, and damping forces.

5. Equilibrium deployment occurs when the aerodynamic opening moment is balanced by the restraining mechanical forces.

6. With the rotor stopped, the spring pre-load is sufficient to stow the flap.

7. The electromagnet is re-energized as part of a normal starting sequence. 
For the $45 \%$ chord spoiler-flap, it was determined that hinge location A-4 was not far enough aft to provide sufficient aerodynamic opening moments. As the WSU wind-tunnel test did not include the hinge location A-5 for the $45 \%$ spoiler, data from hinge locations A-3 and A-4 were extrapolated to estimate the device characteristics at intermediate positions. The best deployment characteristics were found at a hinge location midway between A-4 and A-5, hereafter referred to as hinge location A-4.5. Figure 5-4 shows the $C_{h}-\alpha$ curves for a $45 \%$ chord spoiler-flap hinged at A-4.5. Figures 5-5 show the $\mathrm{C}_{\mathrm{P}}$-TSR curves for this device with a $17.5 \%$ span. As indicated on the figures, these curves assume a $15 \%$ knock-down for finite aspect ratio.

Table 5-2 shows some of the normal points of operation for the AWT-26 turbine, and the general aerodynamic condition of the blade-tip sections. Table 5-2 and Figure 5-4 can be used together to develop an envelope of operational requirements for aerodynamic deployment of the spoiler-flap. This process can provide much insight into the design issues involved.

First note that for blade angles near $\alpha=0^{\circ}$ the hinge moment coefficients are positive and strictly increasing with device deflection angle. As the spoiler is hinged fairly far aft, this is easily understood as being similar to a car door swinging open in a strong wind. The further the door opens, the larger the opening forces become. The spoiler-flap would see this condition during a normal low-wind stop. For this case the dynamic pressure is high due to the rotor's rotation and the blade angle of attack is small due to the low wind speed. This case established a lower limit for the restraining spring torque and damping rates required to prevent a hard mechanical stop of the device. Alternately, an impact stop of the device may be deemed acceptable if properly cushioned.

Next, observe that the device hinge moment coefficients at small deflection angles are small. For $\delta=0^{\circ}, \mathrm{C}_{\mathrm{h}}$ remains below 0.05 for angles of attack up to $15^{\circ}$. The low values of hinge moment coefficients in this range determines the maximum amount of spring pre-load that will allow the device to begin deployment. This constraint will be shown to conflict with the requirements for normal highwind restarts (rotor parked and blades at $\alpha \approx 90^{\circ}$ in high winds). Figure 5-4 shows that the hinge moment coefficients are a maximum at small device deflection angles and $\alpha=90^{\circ}$. In this case the device is acting similar to a weather vane, with the aerodynamic forces resisting closing. Although dynamic pressures are greatly reduced due to the lack of rotor rotation, this case establishes the amount of spring pre-load needed to close the device for restart in high winds.

Inspection of Figures 5-5 shows that the $17.5 \%$ span spoiler-flap is adequate for overspeed protection only if the flap is deployed to at least $75^{\circ}$ (assuming the $15 \%$ knock-down applied). This will determine the amount of restoring spring torque that is allowable at moderate-to-large deflection angles. Figure 5-4 shows that for $60^{\circ}<\delta<90^{\circ}$ the hinge moments coefficients change rapidly with $\alpha$ for $5^{\circ}<\alpha<30^{\circ}$. Unfortunately, the freewheeling condition must be assumed to occur over a wide range of wind and rotor speeds, which correspond to a large range of blade angles of attack and associated dynamic pressures. The steep slope of the hinge moment coefficients in this $\alpha$ range adds to the challenge of designing restoring springs and dampers which allow adequate device deployment for all design cases. The above discussion illustrates many of the issues and constraints in the design of aerodynamically deployed spoiler- flaps, and demonstrates the need for a code capable of rapid deployment simulations. 
Table 5-2. Blade-Tip Aerodynamics for Normal Operation of AWT-26/27

\begin{tabular}{ll}
\hline Normal Mode of Operation & \multicolumn{1}{c}{ Aerodynamic Conditions of Blade-Tip Sections } \\
\hline High-wind stop & $\begin{array}{l}\text { Maximum operating dynamic pressure, the resultant of } \mathrm{V}_{\circ} \text { and } \mathrm{R} \cdot \Omega . \\
\text { High blade angle of attack, } 15^{\circ} \text { to } 20^{\circ} .\end{array}$ \\
Low-wind stop & $\begin{array}{l}\text { Dynamic pressure still high, with large } \mathrm{R} \cdot \Omega \text { component but small } \\
\text { contribution from } \mathrm{V}_{\mathrm{o}} .\end{array}$ \\
& Low blade angle of attack, $0^{\circ}$ to $3^{\circ}$. \\
Stopped in high winds & $\begin{array}{l}\text { Dynamic pressure entirely due to } \mathrm{V}_{\circ}, \text { which may significantly } \\
\text { exceed the turbine cut-out wind speed. }\end{array}$ \\
& Blade angle of attack $\approx 90^{\circ}$. \\
Restart in high winds & $\begin{array}{l}\text { Dynamic pressure entirely due to } \mathrm{V}_{\circ}, \text { which is likely in the range } \\
\text { from } 17.9 \text { to } 20.1 \mathrm{~m} / \mathrm{s}(40 \text { to } 45 \mathrm{mph}) . \\
\text { Blade angle of attack } \approx 90^{\circ} .\end{array}$ \\
\hline
\end{tabular}

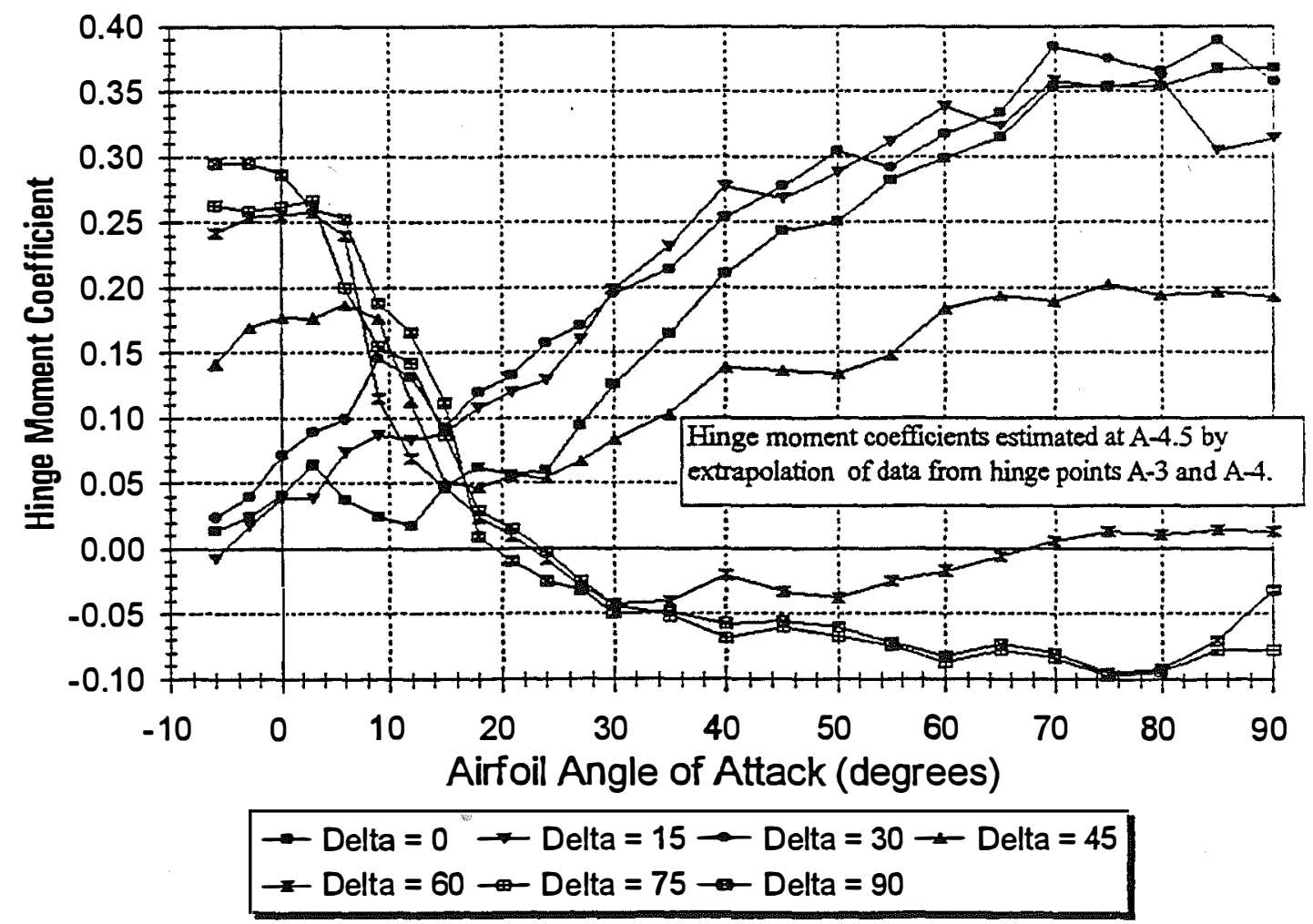

Figure 5-4. Estimated Hinge Moment Data for $45 \%$ Chord Spoiler-Flap at Hinge Location A-4.5 


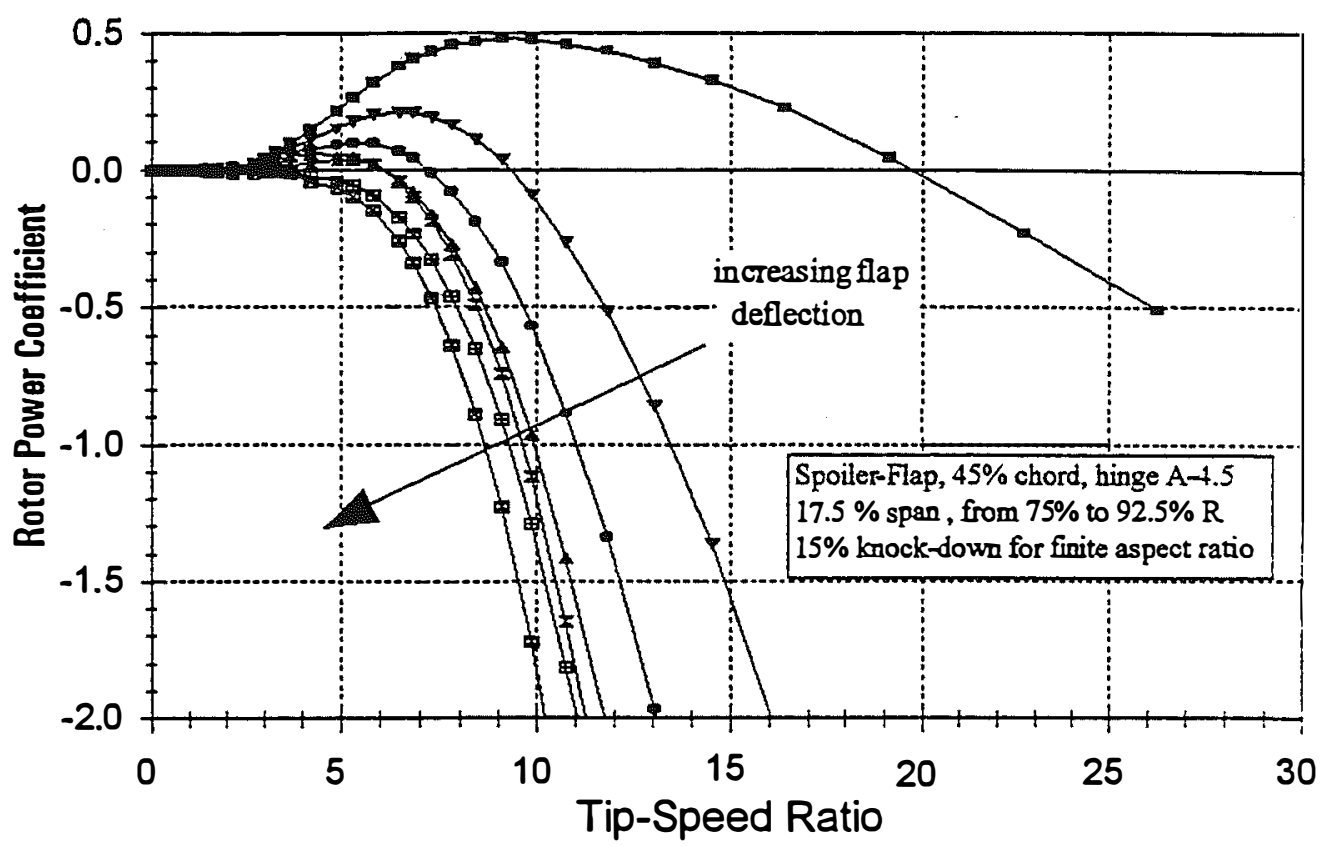

$\rightarrow$ Delta $=0 \rightarrow$ Delta $=15 \rightarrow$ Delta $=30 \rightarrow$ Delta $=45$

$\rightarrow$ Delta $=60 \rightarrow$ Delta $=75 \rightarrow$ Delta $=90$

Figure 5-5a. Rotor Power Coefficients for Deployed Spoiler-Flap (full TSR range)

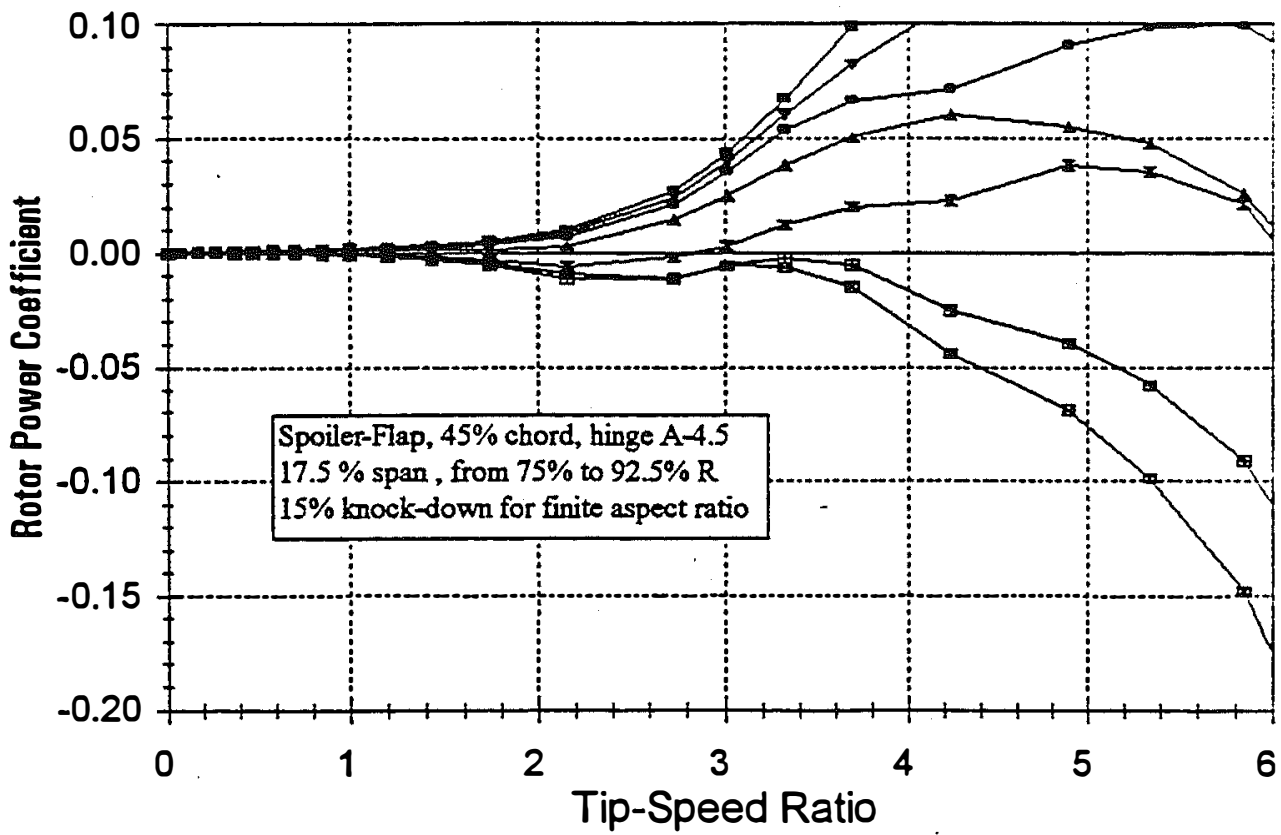

$$
\begin{aligned}
& \rightarrow \text { Delta }=0 \rightarrow \text { Delta }=15 \rightarrow \text { Delta }=30 \rightarrow \text { Delta }=45 \\
& \rightarrow \text { Delta }=60 \rightarrow \text { Delta }=75 \rightarrow \text { Delta }=90
\end{aligned}
$$

Figure 5-5b. Rotor Power Coefficients for Deployed Spoiler-Flap (truncated TSR range) 
Figures 5-6 and 5-7 show two examples of data from deployment time-history simulations. In each case the spoiler-flap is a $45 \%$ chord, $17.5 \%$ span device hinged at location A-4.5. The initial conditions were representative of an AWT-26 turbine that begins freewheeling in wind speeds near cutout, and subsequently experiences a 1-year extreme operating gust. Table 5-3 shows the spring preload, spring-force, and damping schedules used for each simulation. Note that the spring and damping schedules are given in torsional units applied at the hinge. In practice, the restraining and damping torque could either be supplied by torsional devices or by linear devices acting with lever-arms about the hinge line.

Figure 5-6 demonstrates the result of excessive spring rate and inadequate damping. During the first 30 seconds of the simulation the wind condition is held constant. However, the device does not reach a stable equilibrium. This cyclic behavior illustrates the coupling between the rotor speed and the aerodynamically deployed spoiler. As the rotor decelerates, the blade angle of attack increases by several degrees and the dynamic pressure drops. Due to the steep slope of the $C_{h}-\alpha$ curves, the change in $\alpha$ leads to a significant drop in the hinge moment coefficient. The combination of lower hinge moment coefficients and dynamic pressure causes the aerodynamic hinge moments to decrease, and the spring-force begins to close the device. As the device closes, its stopping power is diminished to the point where the rotor begins to accelerate. The accelerating rotor causes decreasing angles of attack and increasing dynamic pressure, and the cyclic behavior continues.

An extreme cycle is initiated by the 12-second cosine gust, which begins 30 seconds into the simulation. During the gust, the rotor accelerates to $96 \mathrm{rpm}$ which greatly exceeds the maximum allowable rotor speed of $75 \mathrm{rpm}$. The simulation shows an emergency application of the mechanical brakes at 45 seconds which stops the rotor completely. As the rotor is stopped, the flap angle approaches $10^{\circ}$ asymptotically, indicating that the spring pre-load is not quite sufficient to stow the device at a wind speed of $22.3 \mathrm{~m} / \mathrm{s}$.

Figure 5-7 shows a near-optimal simulation of freewheeling rotor control by aerodynamically deployed spoiler-flaps. After an initial overspeed to near $75 \mathrm{rpm}$, the flaps deploy sufficiently to slow the rotor. The rotor and flap quickly reach an equilibrium condition, with the rotor speed stable at $61 \mathrm{rpm}$ and the flap deployed to $\delta=70^{\circ}$. During the cosine gust, the rotor accelerates but still remains below $75 \mathrm{rpm}$. Inspection of Table 5-3 shows that the spoiler of Figure 5-7 had a slightly lower spring rate and significantly more damping. This configuration was run with a wide variety of initial conditions, and in each case the maximum rotor speed remained below $75 \mathrm{rpm}$.

Table 5-3. Spring and Damping Torque Schedule for Spoiler-Flap Deployment Simulations

\begin{tabular}{ccccc}
\hline $\begin{array}{c}\text { Spoiler-Flap } \\
\text { Deflection } \\
(\text { degrees })\end{array}$ & $\begin{array}{c}\text { Spring Torque } \\
(\mathrm{N} \cdot \mathrm{m})\end{array}$ & $\begin{array}{c}\text { Figure 5-6 } \\
\text { Damper Torque } \\
(\mathrm{N} \cdot \mathrm{m} \cdot \mathrm{s} / \mathrm{deg} \text { ree })\end{array}$ & $\begin{array}{c}\text { Spring Torque } \\
(\mathrm{N} \cdot \mathrm{m})\end{array}$ & $\begin{array}{c}\text { Damper Torque } \\
(\mathrm{N} \cdot \mathrm{m} \cdot \mathrm{s} / \mathrm{deg} \text { (ee) })\end{array}$ \\
\hline 0 & 13.5 & 0.34 & 13.5 & 1.24 \\
10 & 18.0 & 0.34 & 14.9 & 1.24 \\
20 & 22.6 & 0.34 & 16.4 & 1.24 \\
30 & 27.0 & 0.34 & 17.8 & 1.24 \\
40 & 31.6 & 0.34 & 19.2 & 1.24 \\
50 & 36.1 & 0.34 & 20.6 & 1.24 \\
60 & 40.6 & 0.34 & 22.0 & 1.24 \\
70 & 45.1 & 0.34 & 23.4 & 1.58 \\
80 & 84.6 & 0.34 & 68.5 & 1.58 \\
90 & 124.1 & 0.34 & 113.9 & 1.58 \\
\hline
\end{tabular}




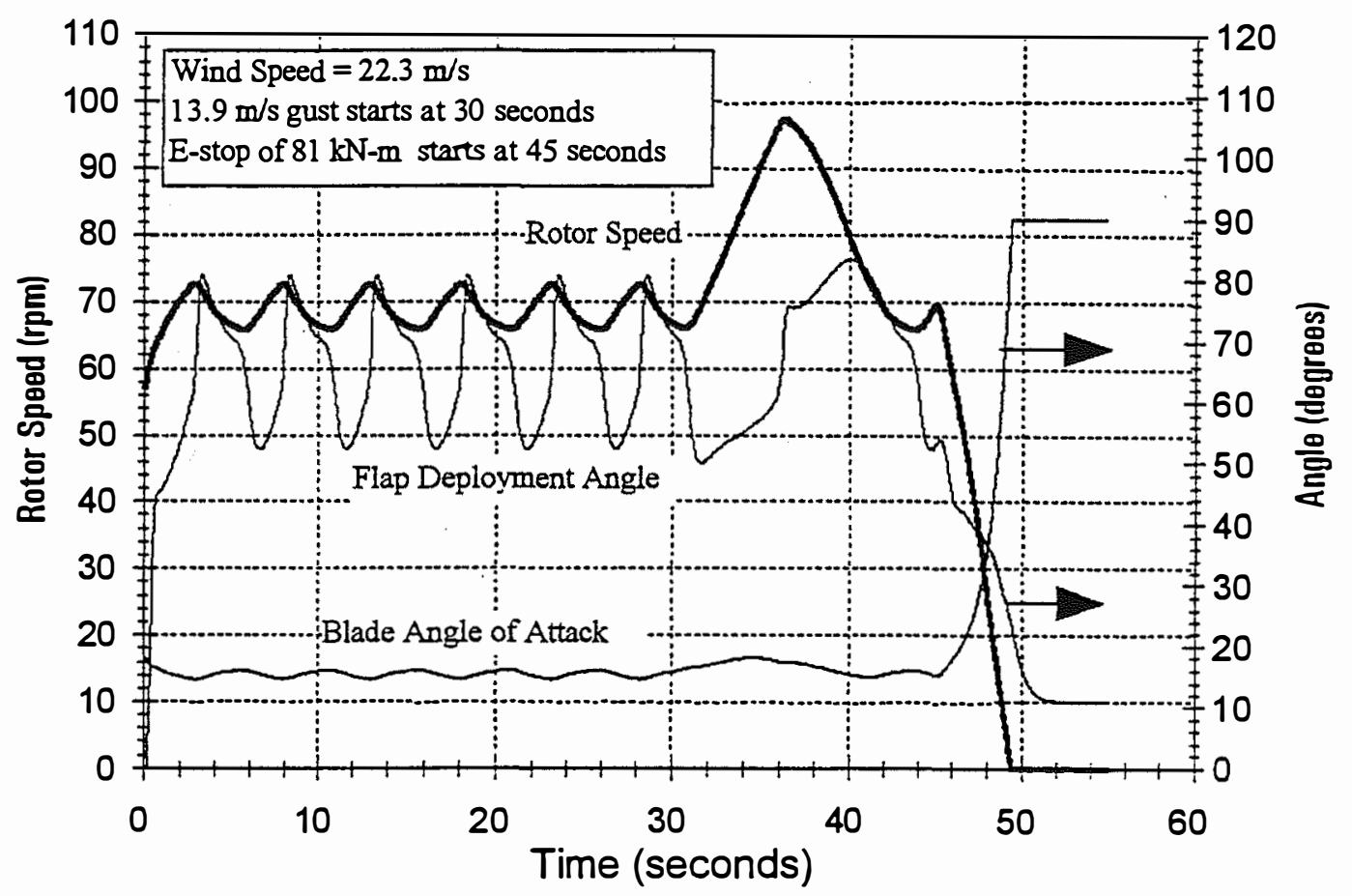

Figure 5-6. Improper Spring and Damping Rates for Aerodynamically Deployed Spoiler-Flap

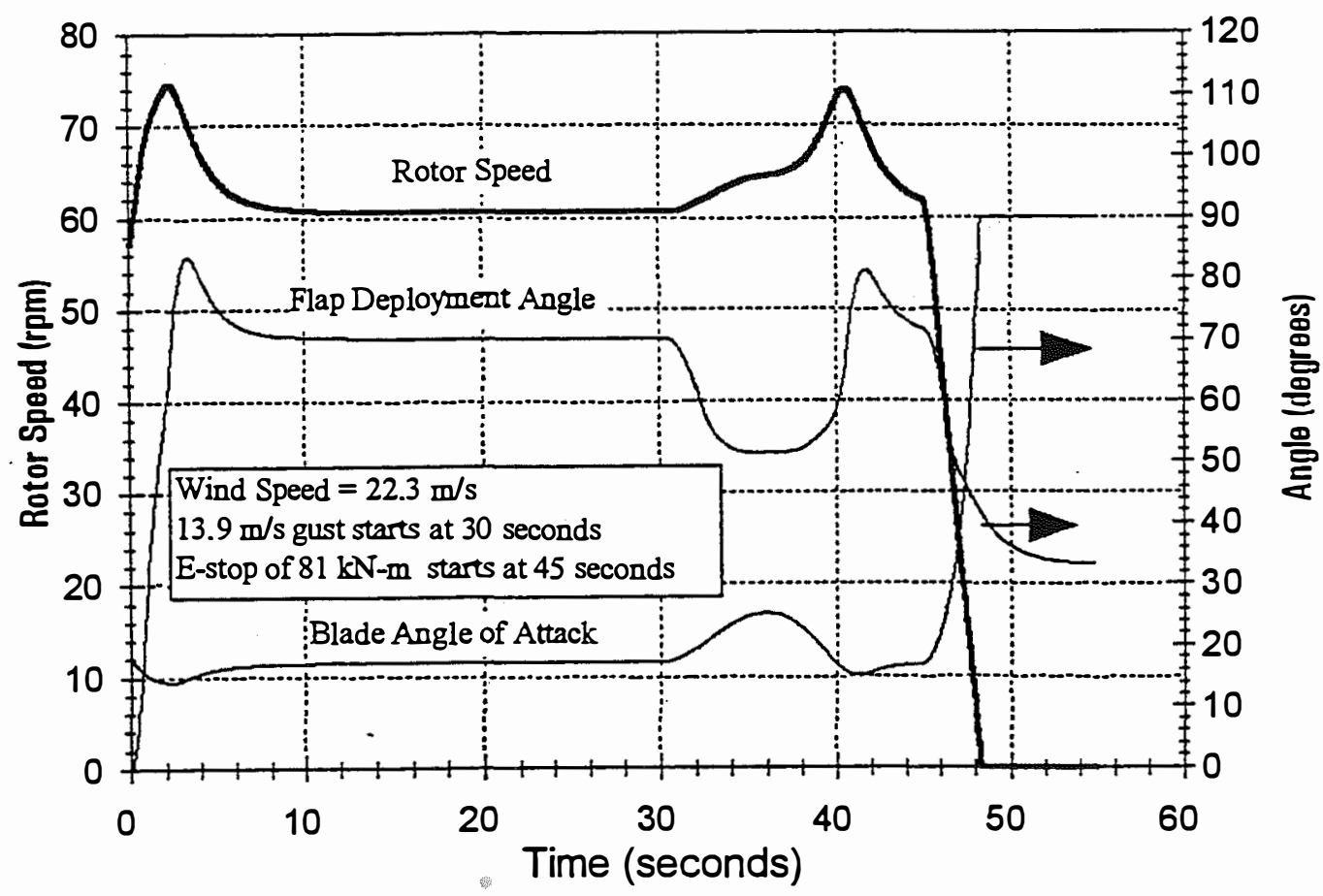

Figure 5-7. Near-Optimal Spring and Damping Rates for Aerodynamic Deployment of Spoiler-Flap 
Inspection of the above figures shows that the deployment behavior could be improved by the use of dual-rate dampers that provide more resistance to closing than to opening. This would be particularly beneficial in reducing the oscillations seen in Figure 5-6. Note that the spring torque schedules for both Figures 5-6 and 5-7 show a significant change in rate at $\delta=70^{\circ}$. Below $70^{\circ}$, the low spring torque allows the device to achieve the large deployment angles required for effective braking. Above $70^{\circ}$, the rate was increased to prevent a hard stop of the device at $\delta=90^{\circ}$.

\subsubsection{Flip-Tip}

Figures 5-8 show $\mathrm{C}_{\mathrm{P}}$-TSR curves for a deployed flip-tip with $12 \%$ total device span. As was shown in Section 5.2, the flip-tip shows excellent stopping power. This is not surprising since the device is essentially an aerodynamically balanced pitchable tip. For the configuration of Figure 5-8, the tips need only deploy to $\delta=60^{\circ}$ to provide adequate overspeed protection.

The flip-tip was conceived as a device with favorable aerodynamic deployment characteristics. However, accurate prediction of the flip-tip's deployment may prove to be difficult. For tip and flap sections of low aspect ratio, the mutual aerodynamic influence of these regions may be large. Methods that rely on linear superposition of hinge moments from the tip and flap regions may therefore be erroneous. The correct aerodynamic balance between tip and flap regions would most easily be obtained through field testing, with bolt-on tip sections available in a range of sizes and/or geometries.

Several simulations were run for flip-tip deployments. A general result of the flip-tip simulations was that the device could only be partially aerodynamically balanced, due to the large range of $\alpha$ and $\delta$ through which the device must operate. As a result, the flip-tip was determined to have aerodynamic deployment characteristics that are less favorable than expected, with mechanical damping requirements similar to the spoiler-flap.

\subsection{Active Mechanical Deployment}

In this discussion a deployment system is considered to be active mechanical if the primary source of opening and closing moments is positively controlled. Active mechanical devices may be driven by hydraulics, pneumatics, or linear or rotary actuators. Conversely, a passive mechanical system would lack an actively controlled source of torque. The passive system would primarily use aerodynamic forces, centripetal accelerations, springs, and dampers.

In principle, the flip-tip could be deployed by either active or passive mechanical methods. However, a major attribute of the device is its potential for favorable aerodynamic deployment. The following sections, therefore, will focus on mechanical deployment of the spoiler-flap device. Although these deployment methods have been treated in separate sections, it is understood that a continuum of design features is possible. For example, an active mechanical system can be designed to take advantage of available aerodynamic forces, and would likely contain one or more passive elements.

For braking and overspeed protection, the most natural way to use active mechanical deployment is to drive the device to a deployment angle at which the full aerodynamic brake effectiveness is realized. To minimize the required actuation forces, a neutral hinge location is desired for active deployment. Hinge moment data are shown in Figures 5-9 for two possible locations. The data show coefficients for both hinge points A-3 and a point halfway between A-3 and A-4, hereafter referred to as hinge point A-3.5. Figure 5-9a shows that hinge location A-3 is very well balanced at deflection angles up to $45^{\circ}$, 


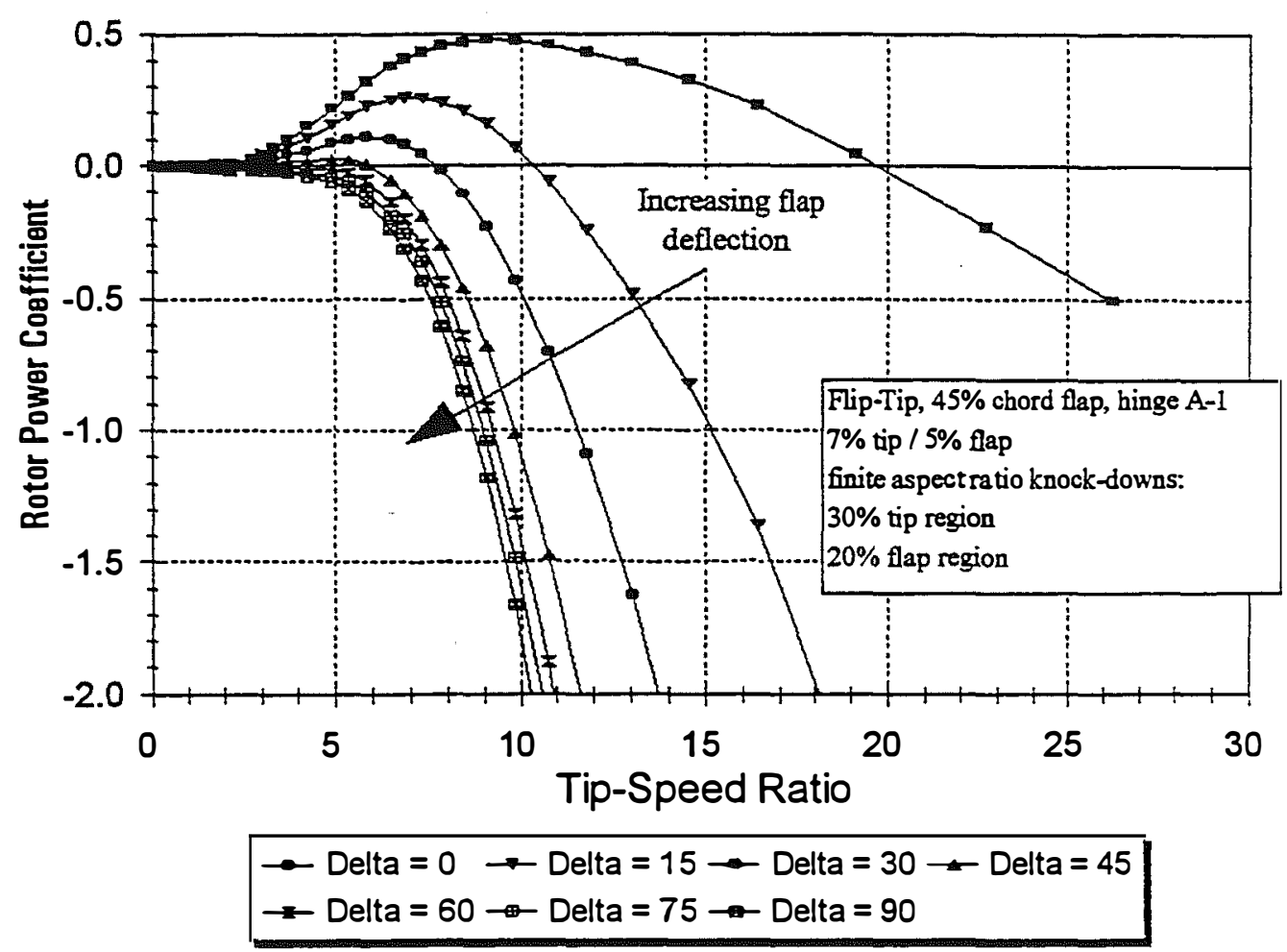

Figure 5-8a. Rotor Power Coefficients for Deployed Flip-Tip (full TSR range)

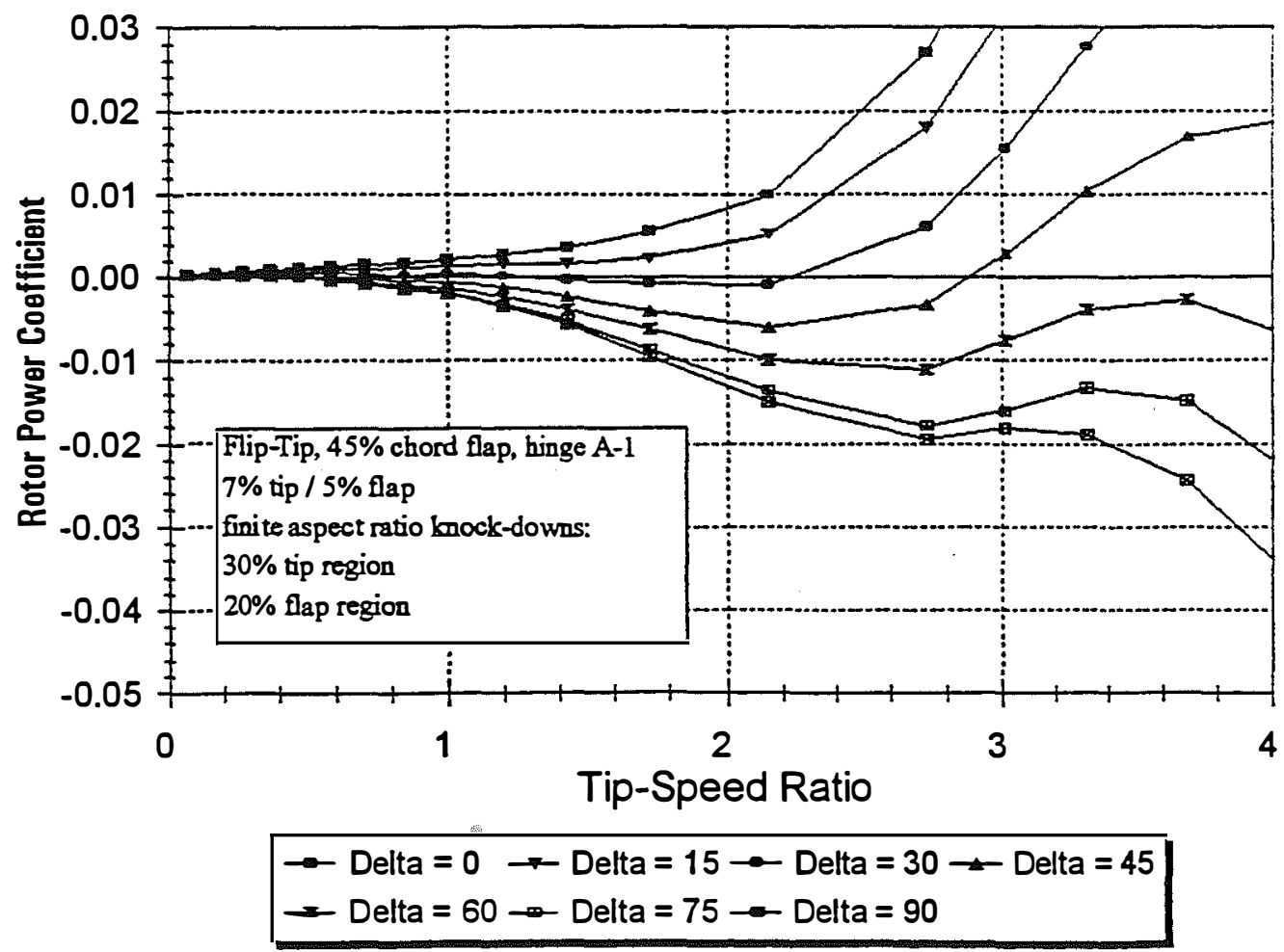

Figure 5-8b. Rotor Power Coefficients for Deployed Flip-Tip (truncated TSR range) 


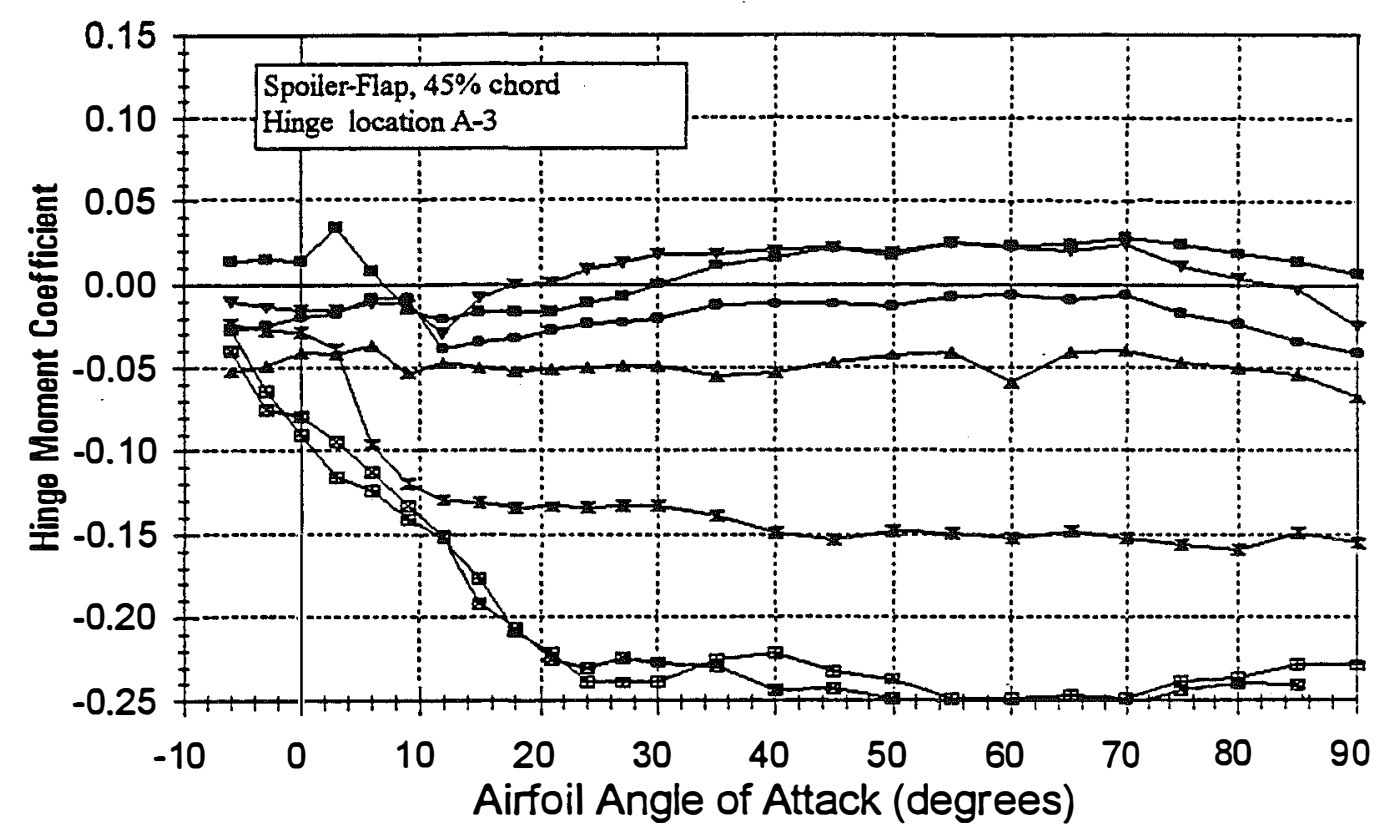

$$
\begin{aligned}
& \rightarrow \text { Delta }=0 \rightarrow \text { Delta }=15 \rightarrow \text { Delta }=30 \multimap \text { Delta }=45 \\
& \rightarrow \text { Delta }=60 \rightarrow \text { Delta }=75 \rightarrow \text { Delta }=90
\end{aligned}
$$

Figure 5-9a. Hinge Moment Data for Balanced Spoiler-Flap, Hinge A-3

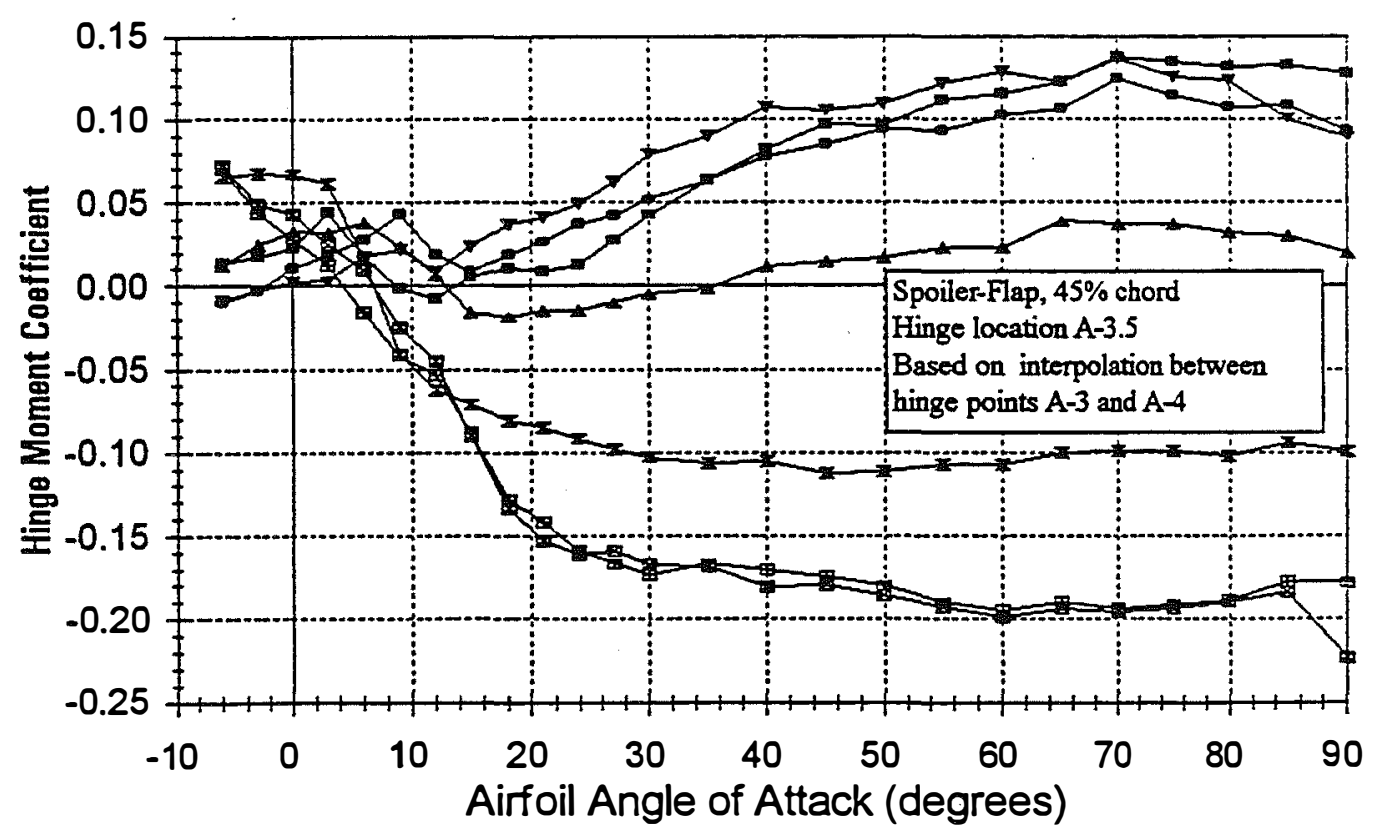

$$
\begin{aligned}
& \rightarrow \text { Delta }=0 \rightarrow \text { Delta }=15 \rightarrow \text { Delta }=30 \rightarrow \text { Delta }=45 \\
& \rightarrow \text { Delta }=60 \rightarrow \text { Delta }=75 \rightarrow \text { Delta }=90
\end{aligned}
$$

Figure 5-9b. Hinge Moment Data for Balanced Spoiler-Flap, Hinge A-3.5 
then tends towards closing hinge moments at higher deflection angles. Hinge location A-3.5 shows a higher tendency towards opening moments, particularly at small deflection angles.

Figure 5-10 shows $C_{P}$-TSR data for a $45 \%$ chord spoiler-flap with $17.5 \%$ span, hinged at location A-3. Note that these curves assume a $10 \%$ knock-down of the two-dimensional data for finite aspect ratio. The $C_{P}$ curves for the device at location A-3.5 are very similar to Figure 5-10. Inspection of the $C_{P}$-TSR curves show that adequate braking and overspeed protection will be achieved when the device is deployed to $\delta \geq 75^{\circ}$.

Data from Figures 5-9 and 5-10 can be used to develop an envelope of actuation torque required for active mechanical deployment. This is shown in Table 5-4 for a spoiler-flap of $17.5 \%$ span which extends from $72.5 \%$ to $90 \% \mathrm{R}$. The table shows that the actuator must supply $333.3 \mathrm{~N}-\mathrm{m}$ of opening torque to sufficiently deploy the spoiler hinged at location A-3. Moving the hinge line aft to location A-3.5 would decrease the actuator torque requirement to $242.2 \mathrm{~N}-\mathrm{m}$. In both cases, the torque required to keep the device closed during normal turbine operation is relatively small.

An additional feature of active mechanical deployment is that it is compatible with the modulation of turbine power. Even at small deployment angles the spoiler-flap efficiently reduces lift and creates drag. This would allow for the use of spoiler-flaps to regulate peak turbine power. Noise impact and behavior under icing conditions are likely to be two primary issues in the design of spoiler-flaps for active power control.

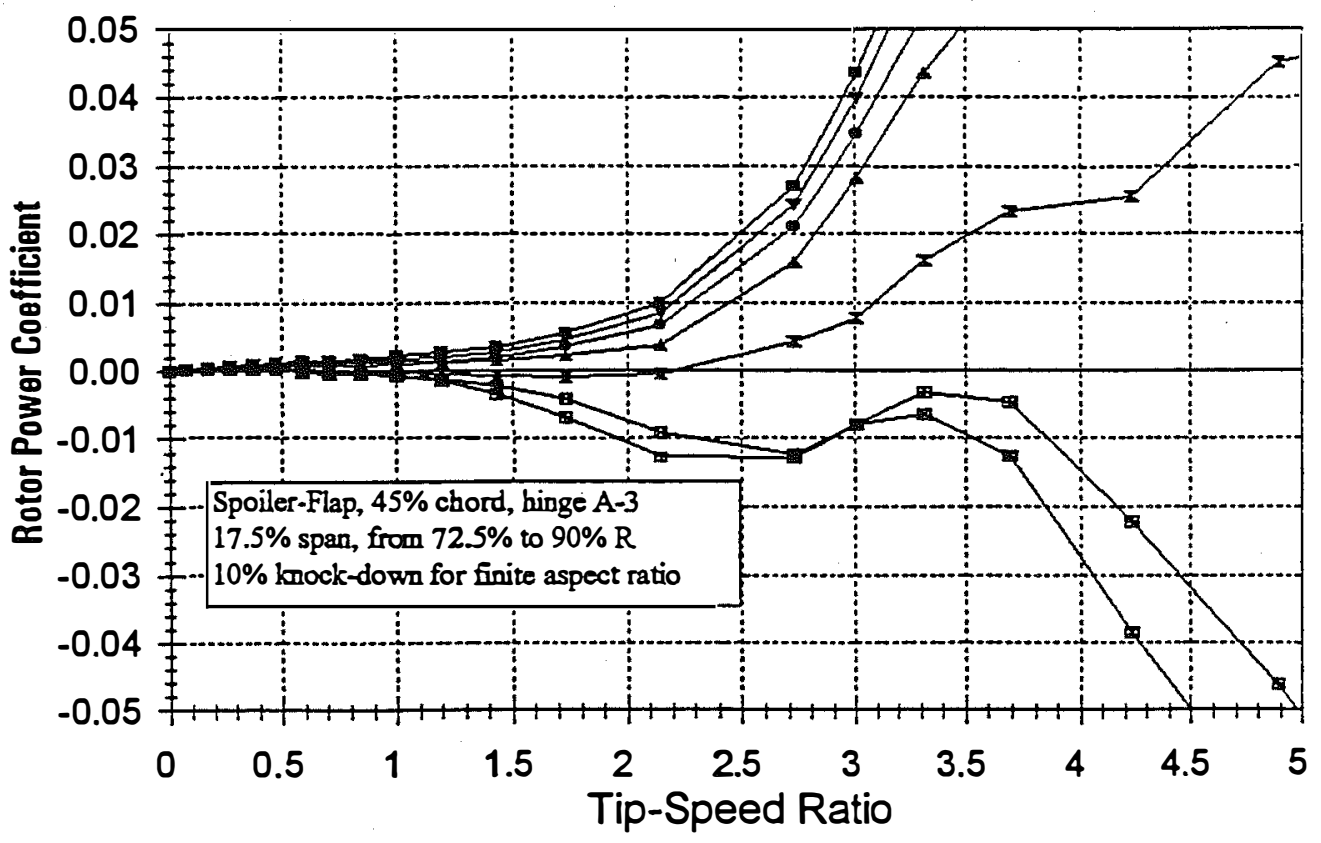

$$
\begin{aligned}
& \rightarrow \text { Delta }=0 \rightarrow \text { Delta }=15 \rightarrow \text { Delta }=30 \rightarrow \text { Delta }=45 \\
& \rightarrow \text { Delta }=60 \rightarrow \text { Delta }=75 \rightarrow \text { Delta }=90
\end{aligned}
$$

Figure 5-10. Rotor Power Coefficients for Deployed Spoiler-Flap (truncated TSR range) 
Table 5-4. Actuation Requirements for Active Mechanical Deployment of Spoiler-Flap

\begin{tabular}{|c|c|c|c|c|}
\hline \multirow{2}{*}{$\begin{array}{c}\text { Operational Mode, } \\
\text { System Requirements, and Defining Parameters }\end{array}$} & \multicolumn{2}{|c|}{ Hinge A-3 } & \multicolumn{2}{|c|}{ Hinge $A-3.5$} \\
\hline & $\mathrm{C}_{\mathrm{h}}$ & $\mathrm{M}_{\mathrm{h}}(\mathrm{N}-\mathrm{m})$ & $\mathrm{C}_{\mathrm{h}}$ & $\mathbf{M}_{\mathrm{h}}(\mathrm{N}-\mathrm{m})$ \\
\hline $\begin{array}{l}\text { Normal power production, device must stay closed } \\
\text { over all operating wind speeds. } \\
\text { Maximum operating dynamic pressure, maximum } \\
\text { positive } C_{h} \text { for } \delta=0^{\circ},-6^{\circ}<\alpha<20^{\circ} \text {. }\end{array}$ & 0.034 & 25.4 & 0.044 & 32.9 \\
\hline $\begin{array}{l}\text { Braking / overspeed protection, device must stay } \\
\text { open to } \delta \approx 75^{\circ} \text { for all wind speeds up to } 36.2 \mathrm{~m} / \mathrm{s} \text {. } \\
\text { Maximum overspeed dynamic pressure, maximum } \\
\text { negative } C_{h} \text { for } \delta=75^{\circ},-0^{\circ}<\alpha<30^{\circ} \text {. }\end{array}$ & -0.238 & -333.2 & -0.173 & -242.2 \\
\hline $\begin{array}{l}\text { Device stowing, actuator must close the device for } \\
\text { high wind restart, wind speeds up to } 20.1 \mathrm{~m} / \mathrm{s} \text {. } \\
\text { Dynamic pressure due to } V_{o} \text {, maximum positive } \\
C_{h} \text { for all } \delta, \alpha=90^{\circ} \text {. }\end{array}$ & 0.007 & 0.6 & 0.128 & 12.6 \\
\hline
\end{tabular}

\subsection{Passive Mechanical Deployment}

This section will outline some of the design issues and possible benefits of passive mechanical deployment, but will not treat the subject in depth. Passive deployment would be similar to the design for active mechanical deployment in that it would likely favor neutral hinge locations with small actuation-moment requirements. Of course, a passive mechanical system may be designed to make judicious use of aerodynamic forces at some points of operation.

The greatest advantage for passive deployment methods on the AWT-26/27 turbines would be realized by eliminating the electrical slip-ring and rectifier, which send power to the electromagnets of the existing tip brakes. As shown in Table 3-1, this system totals $\$ 1,700$ in cost. However, the loss of an electrical circuit across the hub-nacelle interface would require a fundamental change in the braking strategy of the AWT-26/27 turbines. One possibility is to eliminate the use of aerodynamic brakes during normal stopping sequences, using the spoilers only as overspeed protection. This option would increase the turbine's mechanical braking requirements, but could potentially be offset by the savings in eliminating the slip-ring.

\subsection{Configuration Selection for Detailed Design}

The configuration selection process was iterative, with sizing and deployment studies being conducted in parallel with preliminary structural and mechanical design. Although this process was not exhaustive, a large number of device geometries and deployment methods were considered throughout this work. In each case, candidate designs were assessed by their potential for meeting the objectives of Section 1.4, with benefits in the areas of cost, weight, and reliability considered of greatest importance. The following paragraphs summarize some of the general conclusions reached during this process, and describe the configuration selected for detailed design. 
Flip-Tip - The flip-tip showed excellent stopping power which was primarily due to the effectiveness of the tip region. Deployment calculations showed that the aerodynamic damping provided by the flap was favorable, but did not eliminate the need for significant mechanical damping. The correct areaweighting of the tip and flap regions would likely be difficult to predict, but could easily be resolved during a field test program. The major difficulties with the flip-tip device were in the structural design. Significant reinforcement would be required through the flap region and at the tip/flap transition. Structural considerations also favored an external hinge which would potentially be a source of noise and drag. When all advantages and difficulties were considered, it was deemed unlikely that the present flip-tip concept would prove superior to a pure pitchable tip.

Spoiler-Flaps (aerodynamic deployment) - Deployment simulations determined that the spoiler-flap could be designed to deploy aerodynamically, but would require mechanisms to provide specific torsional restraint and damping. Quandt has designed several such spring-damper mechanisms, and is currently testing a prototype aerodynamically deployed spoiler-flap of his own design [7]. However, there are several features of the AWT-26/27 rotor that present difficulties for aerodynamic deployment of spoiler-flaps. First-generation design efforts by both Quandt and AWT engineers showed that the aerodynamically deployed device could be made to work, but may offer little promise in terms of cost and weight benefits.

Spoiler-Flaps (active mechanical deployment) - Considerable structural and mechanical simplicity may be achieved by moving the hinge location forward for active mechanical deployment. Reliability would also be gained by the use of fail-safe mechanisms to drive the spoiler-flap to effective deployment angles $\left(\delta \geq 75^{\circ}\right)$. Initial design work and cost estimates showed that the advantages gained from active deployment may offset some of the additional cost and weight of the actuation system.

Spoiler-Flaps (passive deployment) - Passive deployment on the AWT-26/27 turbines could result in substantial cost savings by eliminating the electrical slip-ring and rectifier. However, the loss of an electrical circuit across the hub-nacelle interface would require a fundamental change in the AWT braking strategy. It is possible that an increase in the turbine's mechanical braking requirements could be offset by the savings in eliminating the slip-ring, although detailed cost trades were not performed during the course of this work.

When all of the above issues had been considered, the spoiler-flap with active mechanical deployment was identified as showing the greatest promise for implementation on the AWT-26/27 turbines. This configuration was thus selected for a detailed design effort, which is presented in the following section. The selected configuration is a $45 \%$ chord spoiler-flap, with $17.5 \%$ total span, located between $72.5 \%$ and $90 \% R$, and hinged at location $A-3$. The assumed $C_{h}-\alpha$ and $C_{P}-T S R$ characteristics of the device are shown in Figures 5-9a and 5-10. 


\section{Design of Spoiler-Flap for AWT-26/27 Rotor}

This section documents the design and analysis of an aerodynamic braking system for the AWT-26/27 rotors. The design process includes the following steps:

- Device sizing

- Loads development

- Trade studies

- Detailed design of selected approach.

As noted in the previous section, the configuration selected for detailed design is a $45 \%$ chord spoilerflap, with $17.5 \%$ total span, located between $72.5 \%$ and $90 \% \mathrm{R}$, and hinged at location A-3. The dimensions and blade station numbers presented in this section are all specific to the AWT-26 rotor blade. A design with the same physical dimensions could be implemented on the AWT-27, but quantities expressed in percent rotor radius would be slightly different.

The actuation system has been designed to ensure device deployment to $\delta=75^{\circ}$. Figure $5-10$ shows that this deployment angle will provide sufficient aerodynamic braking for all design cases, and that deployment angles beyond $75^{\circ}$ show a minimal increase in braking effectiveness. Additionally, $75^{\circ}$ represents the upper limit for mechanical system bellcrank-to-pushrod clearances for the selected approach (Figure 6-3). The maximum opening and closing requirements (specified as torque required at the hinge line) for this configuration are given in Table 5-4.

Once the hinge moment requirements for the actuation system have been specified, many different approaches may be pursued. Driving devices may be hydraulic, pneumatic, or electromechanical. Devices can provide torque directly, or a linear driver may be arranged to act with a lever-arm about the pivot point. For a linear driver, mechanical advantage may be gained through the use of levers, linkages, cams, and cranks. Linear drivers typically scale with their force capacity. A large driver can be located at the hub and simultaneously actuate both aerodynamic brakes through the use of tierods or cables. This arrangement ensures that the devices will deploy together, may decrease the outboard weight on the blade, and may have the benefit of one large driver being less expensive than two (or more) smaller drivers. If linear drivers are located at the flap, a chordwise orientation can be used, but may cause problems in terms of fitting the actuator into the available blade structure. Considerable freedom may be gained by orienting a linear driver in the spanwise direction and using a bellcrank to turn the linear action into the chordwise direction.

Many of the above options were considered during the course of this work, with initial assessment of cost, weight, structural issues, and reliability. The scope of this project did not allow a rigorous costbenefit analysis of the potential actuation methods. The design presented in this section represents one of many ways in which spoiler-flaps may be implemented on the AWT-26/27 turbines.

\subsection{Mechanical Design}

This section presents the design of a pneumatically closed, centripetally opened spoiler-flap. The pneumatic system was chosen versus a hydraulic system due to the low force requirements of the actuator. Initial comparisons of pneumatic and hydraulic system components show that the pneumatic system would be lower cost but higher weight than the equivalent hydraulics. 
Typical operating pressure for the pneumatic system is 150 psi versus 1200 psi for a hydraulic system. To supply a given force, the pneumatic system would therefore require a larger actuator cylinder. In addition, pneumatic pumps and air reservoirs are generally bulkier than their hydraulic counterparts. In terms of fitting the actuation system components into the available blade structure, the pneumatic system therefore is the more demanding case. If the pneumatics can be sized to fit in the blade structure, then certainly the hydraulics will fit.

An electromechanical actuator could also replace the pneumatic actuator, but this option was not rigorously pursued. One benefit of both the hydraulics and pneumatics is that the design for fail-safe operation is straightforward, with system pressure required to keep the device stowed, and any loss of pressure resulting in device deployment. Electromechanical actuators may prove to be a cost-effective alternative to the pneumatic/hydraulic systems, but the design would need to include appropriate failsafe features.

\subsubsection{Overview of Device Construction}

Figure 6-1 shows the AWT-26 blade planform, including the proposed aerodynamic brake. The figure shows station numbers for the blade root and tip, and for the inboard, outboard, and middle positions of the spoiler-flap. The station numbers shown in Figure 6-1 have been taken from AWT-26 blade drawings, and will be referred to in the sections which follow.

The least-cost manufacturing option for the spoiler-flap would allow the device to be cut away from the blade structure during production, then appropriately retrofitted and reinstalled on the blade. The cutting operation would utilize a template to guide a saw or router. Radiused and/or tapered corners are envisioned (but not shown) to reduce stress concentrations from loads transitioning into the uncut blade shell.

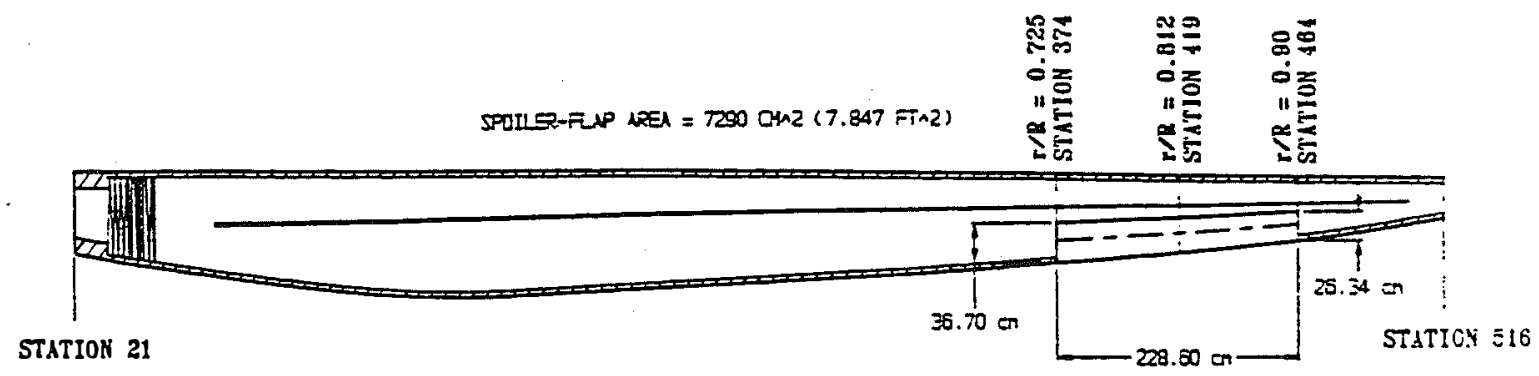

Figure 6-1. AWT-26 Blade Planform with Spoiler-Flap Location Shown

In order to provide structural reinforcement and sealing of exposed internal surfaces and hardware, the blade and device openings are closed out with spanwise webs and chordwise ribs. Webs have been initially sized using $6.35 \mathrm{~mm}$ (0.25 in.) birch plywood, and ribs are initially sized as $12.7 \mathrm{~mm}(0.5 \mathrm{in}$.) thick wood/epoxy laminate. Webs can be cut from plywood sheet stock. Ribs can readily be cut from a parent billet composed of five Douglas Fir veneers $2.54 \mathrm{~mm}(0.1 \mathrm{in}$.) thick, laminated with epoxy at room temperature under pressure. 
For access to system hardware, panels need to be installed in the blade and device webs. In addition, slotted openings in the webs must be cut for the device control linkages. Many of the openings may be cut during initial trimming from the plywood sheet. To reduce leakage from the low-pressure to the high-pressure surface of the blade, the spanwise gaps should be sealed with an appropriate rubber seal. Chordwise gaps are more difficult to seal due to the requirements for device freedom of motion under high loads. The aerodynamic impact (both noise and performance) of through-flow at the device ends would need to be assessed to determine whether a design remedy is warranted.

The device is mounted to the blade using hinge pins at the inboard and outboard edges of the device. Centripetal forces from the device are transferred to the blade structure through a large-area thrust bearing located at the hinge, as shown in Figure 6-2. Bearing loads are shared by the outboard blade rib and the blade laminate, thus reducing concentration of loads into the blade rib and subsequent high shear stresses in the bond-line.

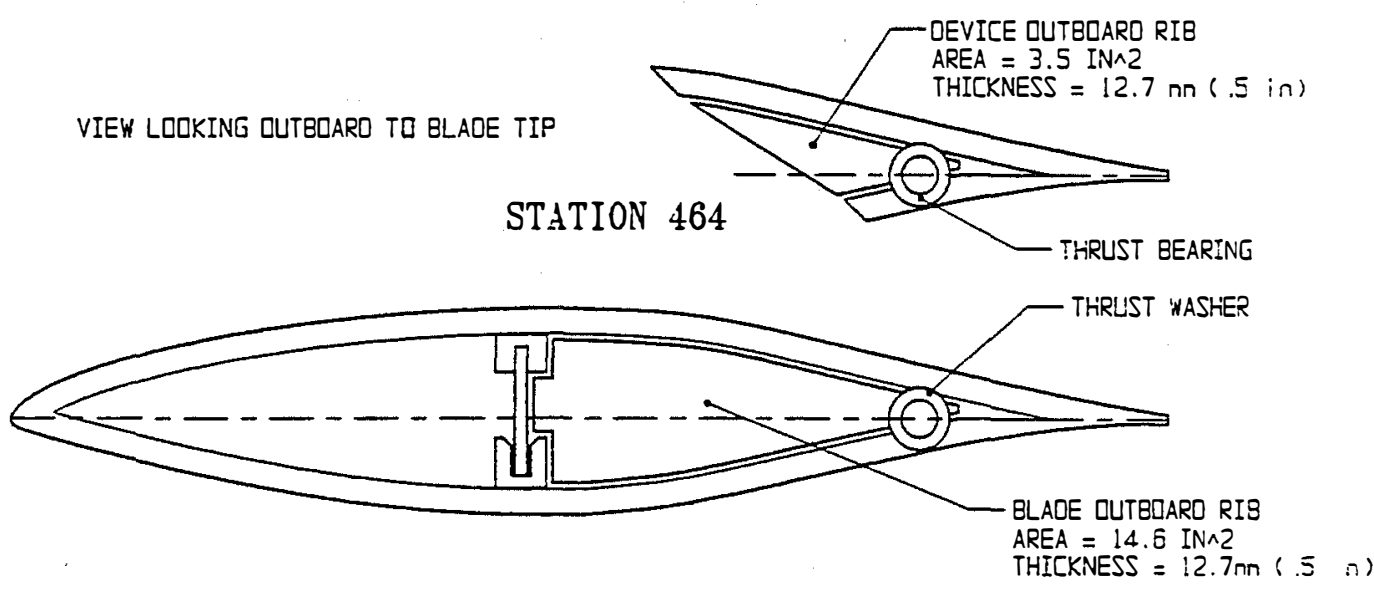

Figure 6-2. Spoiler-Flap Hinge / Thrust Bearing Configuration at Station 464

\subsubsection{Mechanical System Description}

Figures 6-3 and 6-4 provide layout sketches of the mechanical system used to open and close the device. The primary driver consists of an actuator, a bellcrank, and a pushrod that open and close the device at mid-span. Secondary ( passive) drivers are gas springs located at either end of the device. The gas springs help hold the ends open during braking and hold the corners closed during normal turbine operation.

Pneumatic pressure provided by a hub-mounted pump is used to retract the cylinder in each blade, thus closing the device. To reduce cycling of the pump, a hub-mounted air reservoir would be added to the system. To open the device, a pneumatic valve would dump line pressure, allowing the cylinder to extend as a result of the actuator mass subjected to centripetal loading. Due to the high centripetal loads at the 10-meter span location of the actuator during normal and overspeed operating conditions, a $5.5 \mathrm{~kg}$ cylinder mass is capable of driving the device open. For this reason, the actuator pushrod is fixed to the shear web, while the heavier cylinder body is allowed to exert its mass radially outboard during deployment. If the installation is reversed, or if the cylinder body does not have sufficient mass to provide the centripetal force required, additional weight would have to be added to the actuator. 
Both aerodynamic hinge moment and centripetal force change proportionally with the square of rotor rotational speed, so opening force will be maintained throughout the braking envelope. In designs with large overspeed margins, it may be possible to set system pressure such that a large overspeed will overcome the actuator closing force, thus forcing the device open without relying solely upon disconnection of electrical current to the normally-open pneumatic valve.

Two additional design features assist the centripetal operation: the low hinge moment due to the neutral hinge location, and a 2:1 bellcrank ratio that doubles the effect of the actuator centripetal force. Pressure dumping is achieved by using a normally-open pneumatic solenoid valve located in the hub. The valve is electrically closed by the nacelle controller (via the slip-ring) to stow the spoiler for normal turbine operation.

A normal or emergency braking condition would result in disconnection of the pneumatic valve electrical circuit and subsequent centripetal opening of the device as pressure bleeds off. Pressure dump rates can be adjusted to reduce opening shock by using a metered orifice. The system is designed to be fail-safe, as any loss of power would also result in device deployment. Table 6-1 summarizes the actuator specifications required to drive the present spoiler-flap design. A variety of standard clevis ends and mounting brackets are available to facilitate the installation of the actuator.

An additional consideration in the design of the pneumatic system is the prevention of moisture and particulate accumulation during compressor operation. If the pump does not include moisture and particle filters, they will have to be designed into the system. A practical choice might be the miniature "piggyback" filter/regulator, which is available commercially.
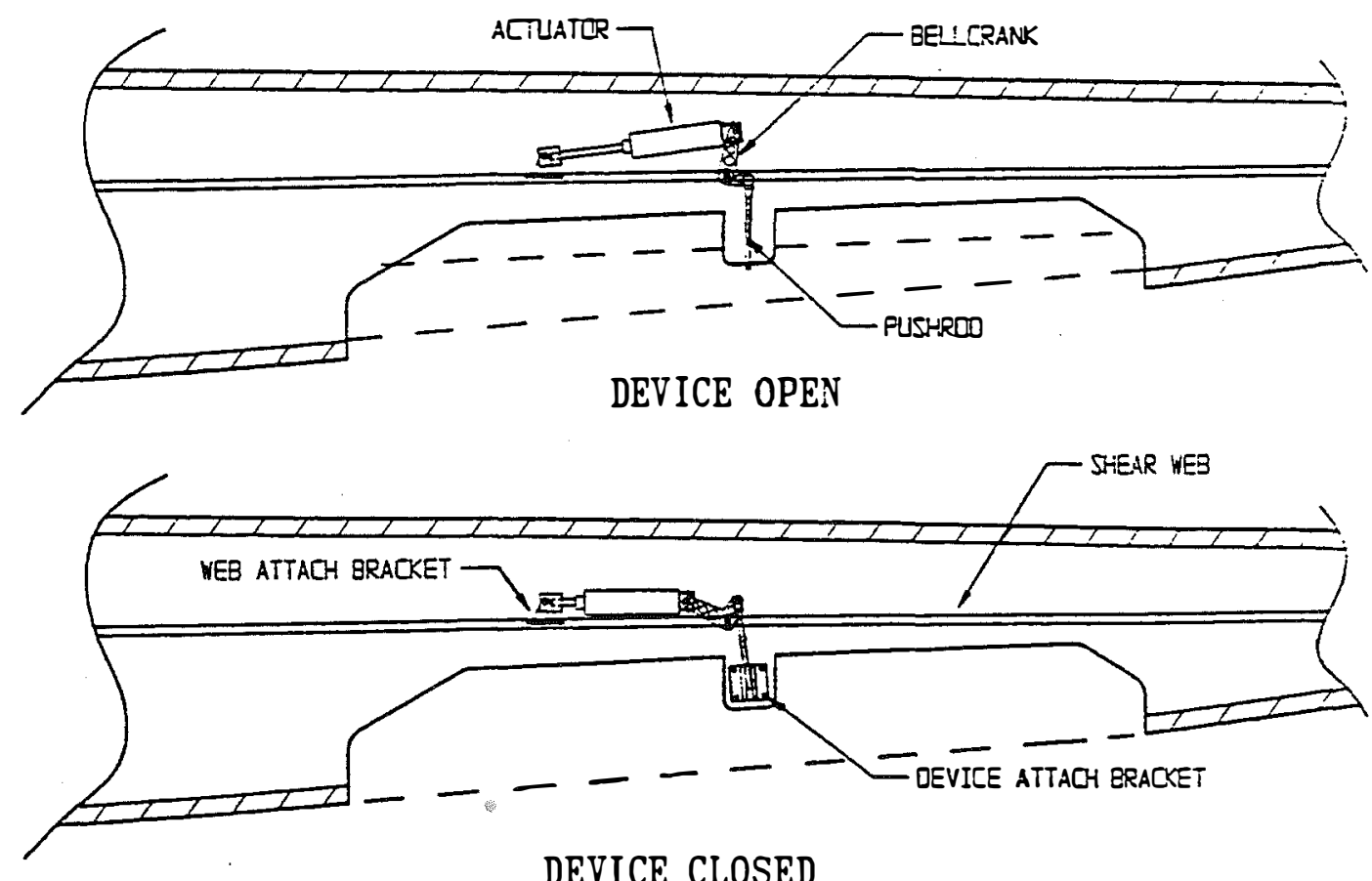

Figure 6-3. Layout of Mechanical Actuation Driver 
Stroke

Bore

Operating pressure

Closing force at $60 \mathrm{rpm}$ rotor speed (normal turbine operation)

Body mass required to drive device open at $75 \mathrm{rpm}$ (overspeed)
$16.8 \mathrm{~cm}$ (6.6 in.)

$6.35 \mathrm{~cm}(2.5 \mathrm{in}$.

$1035 \mathrm{kPa}(150 \mathrm{psi})$

$2277 \mathrm{~N}(512 \mathrm{lb})$

$5.5 \mathrm{~kg}(11.1 \mathrm{lb})$
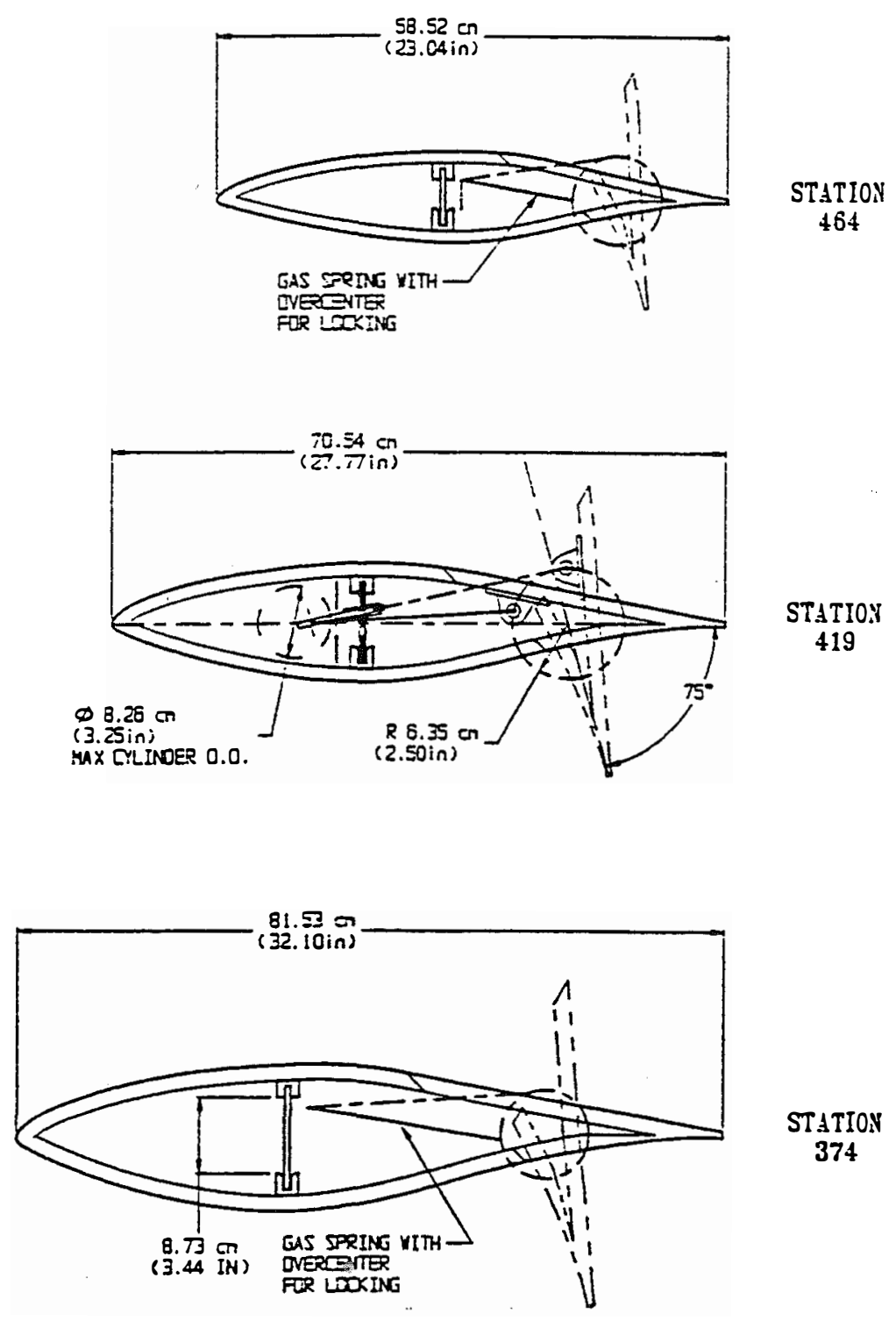

Figure 6-4. Sectional Layout of Mechanical Driver 


\subsection{Loads and Structural Analysis}

For the current preliminary design, only the peak static loads and their effect on blade and device stresses have been examined. Due to the introduction of possible stress raisers at blade discontinuities in the spoiler-flap region, an investigation of blade fatigue would be necessary during the next stage of design effort. Loads and stresses are based upon the geometry described in Section 6.1.

\subsubsection{Blade Loads and Structural Analysis}

For comparative analysis, blade-flapwise and edgewise bending loads are assumed to remain the same as specified for the AWT-26/27 with tip brakes. The inboard edge of the spoiler-flap is the most critical section with respect to blade bending stresses, so the analysis has initially been limited to station 374. The bending loads shown in Table 6-2 have been taken from the AWT-26/27 Loads Design Book [16].

Table 6-2. Blade Bending Loads at Station 374

\begin{tabular}{cccc}
\hline \multicolumn{2}{c}{ Edgewise } & \multicolumn{2}{c}{ Flapwise } \\
$\mathrm{kN}-\mathrm{m}$ & $\mathrm{ft}-\mathrm{kip}$ & $\mathrm{kN}-\mathrm{m}$ & $\mathrm{ft}-\mathrm{kip}$ \\
\hline 35.8 & 26.4 & 25.8 & 19.0 \\
\hline
\end{tabular}

Bending stresses were determined according to:

$$
\sigma=M c / I
$$

where $c$ is the distance from the neutral axis to the extreme fiber and $I$ is the section moment of inertia. Figure 6-5 shows the cross-sectional properties of the blade at station 374 before and after installation of the device.

A summary of tensile and compressive stress increments for the AWT-26 blade are given in Table 6-3. The increments are a function of distance from the neutral axis to the extreme fiber and change in section moment of inertia. Using the constant bending moments, the stress increments were determined from the following ratio:

$$
100 \cdot\left(1-\frac{\sigma_{1}}{\sigma_{2}}\right)=100 \cdot\left(1-\frac{c_{2} \cdot I_{1}}{c_{1} \cdot I_{2}}\right),
$$

where the subscripts 1 and 2 denoted the section properties before and after the modification, respectively. A significant increase in edgewise bending stress occurs. For the AWT- 26 blade, a large edgewise margin of safety exists. Therefore, the structural reinforcement provided by the additional web at the blade trailing edge close-out, shown in Figure 6-5, provides a sufficient, but smaller, margin of safety. Bonding and shear tie details for transfer of tensile loads to the close-out ribs and blade would need to be addressed during a more detailed structural evaluation and design. To reáuce stress concentrations that will affect the fatigue life of the blade at the corners of the spoiler-flap, analysis for the proper radius or taper should be conducted. 
Table 6-3. Blade Percentage Stress Increments at Station 374

\begin{tabular}{cccc}
\hline $\begin{array}{c}\text { Flapwise Tension } \\
\text { (high-pressure shell) }\end{array}$ & $\begin{array}{c}\text { Flapwise } \\
\text { Compression }\end{array}$ & $\begin{array}{c}\text { Edgewise Tension } \\
\text { (trailing edge) }\end{array}$ & $\begin{array}{c}\text { Edgewise } \\
\text { Compression }\end{array}$ \\
\hline $15 \%$ & $23 \%$ & $96 \%$ & $270 \%$ \\
\hline
\end{tabular}
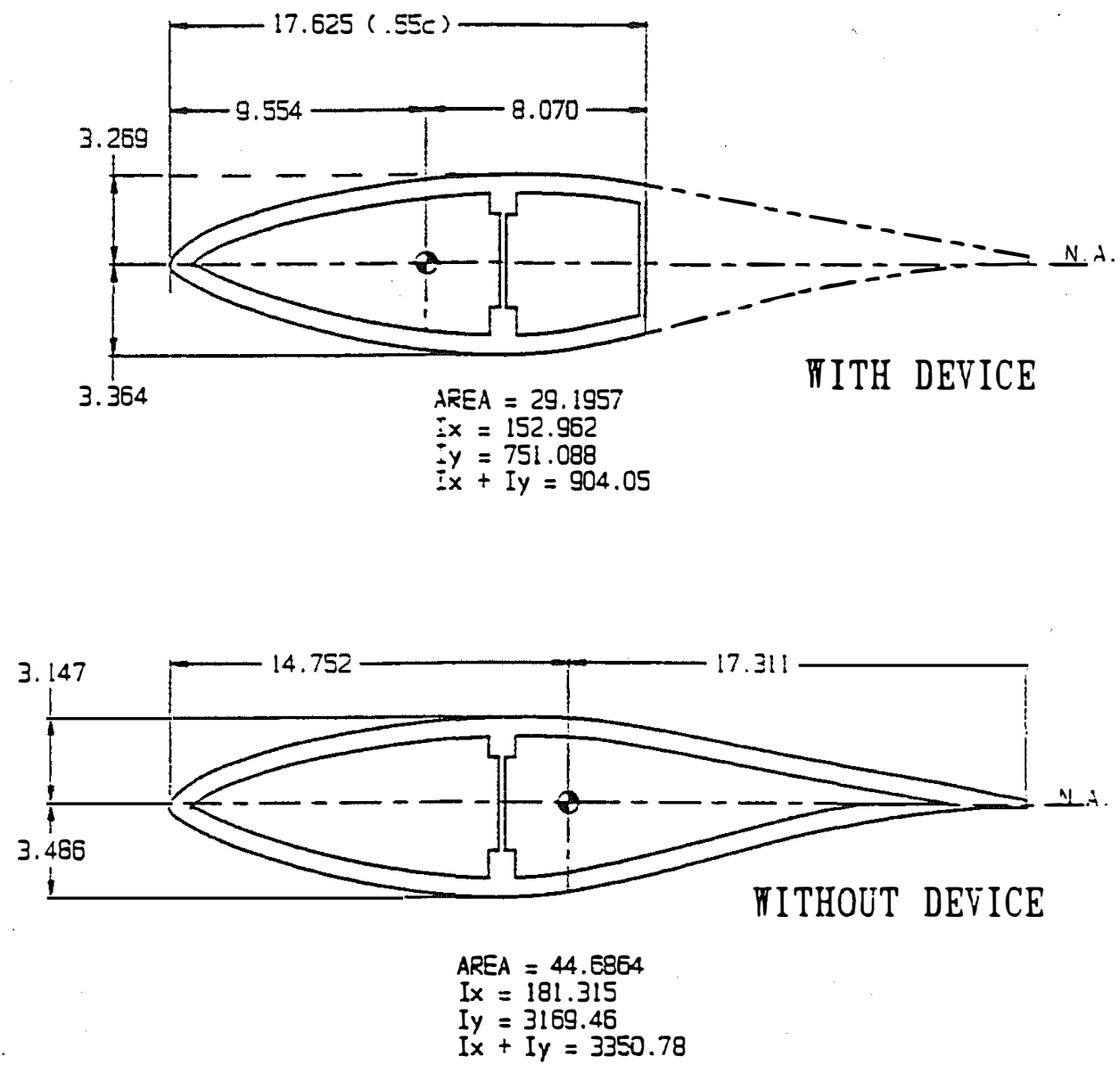

Figure 6-5. Station 374 Blade Section Properties ( all dimensions in inches)

\subsubsection{Device Loads and Structural Analysis}

The rotor overspeed condition causes the highest device loads. Maximum bending loads occur when the device is open. The maximum bending stress results from overspeed deployment combined with pushrod force, and occurs at the point of device actuation at mid-span (station 410). Maximum bearing load is a result of centripetal load transfer from the device to the outboard hinge rib. It occurs during maximum overspeed when centripetal loads are also at a maximum.

The critical device loads are summarized in Table 6-4. Overspeed drag load results from the middevice-span dynamic pressure at $90^{\circ}$ to the free stream with a rotor speed of $75 \mathrm{rpm}$. Pushrod load is that which is required to keep the device open during overspeed, and is determined as the maximum overspeed hinge moment divided by the perpendicular distance from the hinge line to the point of actuation (arm length $=6.35 \mathrm{~cm}$ ). Maximum hinge bearing load is the mass of the device times the centripetal acceleration during overspeed. 
Table 6-4. Device Critical Loads

\begin{tabular}{ccccccccc}
\hline \multicolumn{2}{c}{ Maximum } & \multicolumn{2}{c}{ Maximum Hinge } & \multicolumn{2}{c}{ Maximum Pushrod } & \multicolumn{2}{c}{ Maximum Hinge } \\
\multicolumn{2}{c}{ Drag Load } & \multicolumn{2}{c}{ Moment } & \multicolumn{2}{c}{ Load } & \multicolumn{2}{c}{ Bearing Load } \\
$\mathrm{kPa}$ & $\mathrm{psf}$ & $\mathrm{N}-\mathrm{m}$ & in-lb & $\mathrm{N}$ & $\mathrm{lb}$ & $\mathrm{N}$ & $\mathrm{lb}$ \\
\hline 4.48 & 93.6 & 339 & 3000 & 5338 & 1200 & 10369 & 2331 \\
\hline
\end{tabular}

For device bending stress analysis, the mechanical pushrod load was concentrated at the point of application and the aerodynamic drag loads were uniformly distributed along the device span. Stresses due to each were linearly superimposed, with the resulting critical stresses shown in Table 6-5. This analysis showed that additional structural modification may be needed to reduce the bending stresses to meet design factors of safety. Such modification might include addition of a mid-span rib, or stiffening of the device by either thickening or reinforcing the close-out web. The use of a $32 \mathrm{~mm}$ (1.25 in.) OD $\mathrm{x} 19 \mathrm{~mm}(0.75 \mathrm{in}$.) ID thrust washer to transfer the thrust bearing loads from the device to the blade would provide ample capability for carrying the hinge bearing loads. Accommodation for sealing the thrust bearing from moisture and airborne particles would reduce maintenance requirements on the bearings.

Table 6-5. Device Critical Stresses

\begin{tabular}{cccc}
\hline \multicolumn{2}{c}{ Mid-Span Bending Stress } & \multicolumn{2}{c}{ Hinge Bearing Stress } \\
(overspeed) & & \multicolumn{2}{c}{ (overspeed) } \\
$\mathrm{kPa}$ & $\mathrm{psf}$ & $\mathrm{kPa}$ & $\mathrm{psf}$ \\
\hline 356.6 & 7448 & 142.0 & 2966 \\
\hline
\end{tabular}

\subsection{Cost and Weight Estimates}

Table 6-6 shows the comparative costs and weights of the present design and the existing AWT-26/27 tip vane. AWT-26/27 production tip vane costs are based on 1995 invoices, and weights are taken from measurement of production components. Appendix B contains a spreadsheet that details the estimates of production costs and weights for the spoiler-flap design. The spreadsheet contains notes which document the basis for each estimated cost. Major system components are priced from a single source, assuming part quantities for $100 \mathrm{AWT}$ wind turbines. Other commercially available components are priced from catalogs, less an assumed $30 \%$ for quantity discounts. Where possible, hardware prices are based on AWT production invoices for comparable items. Estimates for custom wood and machined items are based upon existing cost-per-weight of blades and tip-vane components. Labor-hour estimates are based upon current AWT manufacturing experience. Due to the preliminary nature of the spoiler-flap design, additional labor and material costs are likely to occur as a result of detailed design for manufacture. These additional costs are estimated in the spreadsheet by a line item for "unspecified parts and processes."

Table 6-6 shows that production costs for the spoiler-flap design are estimated to be $5 \%$ lower than the baseline AWT-26/27 tip vane. Although this is encouraging, the preliminary level of the current design must be kept in mind. The greatest uncertainty in the cost estimates is the labor required to cut out, retrofit and reinstall the device on the blade. 
Table 6-6. Cost and Weight Analysis Summary

\begin{tabular}{lccc}
\hline & $\begin{array}{c}\text { Installed Cost } \\
\text { (total system) }\end{array}$ & $\begin{array}{c}\text { Blade Component Weight } \\
\text { (per blade) }\end{array}$ & $\begin{array}{c}\text { Hub Component Weight } \\
(1 \text { per turbine) }\end{array}$ \\
\hline Spoiler-Flap & $\$ 2,903$ & $17.6 \mathrm{~kg}(38.8 \mathrm{lb})$ & $16.8 \mathrm{~kg}(37 \mathrm{lb})$ \\
Current Tip Brake & $\$ 3,057$ & $12.7 \mathrm{~kg}(28.0 \mathrm{lb})$ & $4.1 \mathrm{~kg}(9 \mathrm{lb})$ \\
\hline
\end{tabular}

Notes: Slip ring price and weight excluded from both systems.

Rectifier included in cost and weight for tip brake (not needed for spoiler-flap).

Both systems include installation labor.

Table 6-6 shows that the blade components of the spoiler-flap system are heavier than the baseline tip vane. However, the effect of the weight differential is somewhat offset by the device placement. The AWT-26/27 tip-vane weight is concentrated at the blade tip, whereas the spoiler-flap center of mass is further inboard. Therefore, the centripetal loading on the devices will be reduced by a factor of $400 / 516$ (the ratio of device radial locations). The baseline weight of an AWT-26 blade is $454 \mathrm{~kg}$ $(1000 \mathrm{lbs})$, so the $4.9 \mathrm{~kg}(10.8 \mathrm{lb})$ increase in blade component weight would result in an effective increase of $1.1 \%$ in total blade weight, once centripetal effects are included.

The estimated increase in blade component weight is not an anomaly of the current spoiler-flap design. During the design trade-off studies, weights were estimated for several candidate configurations, including aerodynamically deployed spoiler-flaps and flip-tips. In each case, the estimated bladecomponent weights were greater than the baseline AWT-26/27 tip vane. The greatest contributor to increased weight was the need for multiple hinge points and the associated structural reinforcements.

It was concluded that the spoiler-flap and flip-tip configurations did not hold significant promise for absolute reductions in blade component weight. However, small increases in system weight may be acceptable if other advantages are shown (e.g. noise and performance benefits due to improved tip shape).

At this level of analysis, the design appears to be competitive with the baseline configuration on a cost and weight basis. This is encouraging for a first-generation design, as improvements in both cost and weight may be obtained through design iterations. 


\section{Conclusions}

Wind-tunnel tests have been conducted for a large number of plain-flap and spoiler-flap configurations. The wide range of parameters tested, and the quality of the data collected, have provided an excellent database for the present work and for other wind turbine designers who may be considering trailingedge aerodynamic brakes.

The wind-tunnel results have been used to identify the aerodynamic performance and deployment characteristics of candidate configurations. Preliminary design effort on the flip-tip showed limited promise for improvements relative to a classic pitchable tip. Significant non-linearity of hinge-moment behavior was identified for both the flip-tip and spoiler-flap configurations. As a result, it was concluded that pure aerodynamic deployment of either configuration would be difficult to achieve.

The spoiler-flap, with active mechanical deployment, was selected for additional preliminary design effort. The result was a promising configuration for the AWT-26/27, with initial estimates of a small decrease in cost and an increase in weight when compared with the currently-used tip vane. This is encouraging for a first-generation design. However, there are several design issues that would require further work to increase confidence in these results, including:

- Appropriate adjustment for finite aspect ratio and other three-dimensional effects. As discussed in the text, the appropriate adjustments for aspect ratio effects on spoiler-flap data are unknown. The results of References 5 and 6 may provide more information on this issue, but it is unlikely that they will resolve it once and for all. Although this work used a best guess for the three-dimensional adjustments, prototype testing of the devices would be required to confidently establish the device sizing.

- Aeroelastic / dynamic behavior of spoiler-flap and modified blade. In the present design, the modified blade section (forward element) is much stiffer in flapwise bending than the spoiler-flap (rear element). By hinging the flap at both ends and supporting the device mid-span, it is expected that the spoiler deflections would generally follow those of tie blade. This assumption would need to be validated through further analysis and field testing. A significant difference between blade and spoiler deflections could result in a mismatch between the aerodynamic surfaces, which would likely increase noise and decrease turbine performance.

- Tolerances and gap-sealing requirements. The design would require close tolerances on hinge location and orientation, both to ensure smooth deployment (no hinge binding) and proper device alignment. Maintaining flush aerodynamic surfaces and proper sealing of gaps would be necessary to minimize airfoil noise and drag. This could prove difficult on the three-dimensional complex curves of the AWT-26/27 rotor blade, with both taper and twist.

- Greater detail of structural analysis. The analysis of this report was based on loads at extreme operating conditions. Inspection of the operational envelope indicates that these load cases would dominate the design, but this has not been confirmed. Additionally, sizing and structural analysis must be performed for intermediate mechanisms, connections, and hardware, although none of these items are expected to be problematic. 
- Sensitivity of the device performance to icing conditions. This may be particularly important if significant gaps are present at the device ends, as ice accumulation could seal the device closed.

The above issues may be addressed during further design of an aerodynamic braking system. Although none of these issues appear prohibitive, prototype testing of spoiler-flaps would be required to confidently establish the device performance. 


\section{References}

1. Gipe, P., Wind Energy Comes of Age, John Wiley \& Sons, New York, 1995.

2. Eggleston,, D.M., and Stoddard, F.S., Wind Turbine Engineering Design, Van Nostrand Reinhold, New York, 1987.

3. Miller, L.S., Experimental Investigation of Aerodynamic Devices for Wind Turbine Rotational Speed Control: Phase 1, NREL/TP-441-6913, Golden CO: National Renewable Energy Laboratory, February 1995.

4. Miller, L.S., Experimental Investigation of Aerodynamic Devices for Wind Turbine Rotational Speed Control: Phase II, NREL/TP-441-20507, Golden CO: National Renewable Energy Laboratory, February 1996.

5. Ramsay, R.R., Janiszewska, J.M., and Gregorek, G.M., Wind Tunnel Testing of Three S809 Aileron Configurations for use on Horizontal Axis Wind Turbines, Midwest Research Institute Contract Number XF-11009-3, publication pending.

6. Miller, L.S., Quandt, G.A., and Huang, S., Atmospheric Tests of Trailing-Edge Aerodynamic Devices, NREL publication pending.

7. Cheney, M.C., PS Enterprises. Inc., Advanced Wind Turbine Subcontract \# AAA-4-12272-04, Subcontract Report pending.

8. Quandt, G.A., Wind Turbine Trailing-Edge Aerodynamic Brake Design, NREL/TP-441-7389, Golden CO: National Renewable Energy Laboratory, January 1996.

9. Johnson, B.L., Facility Description of the Walter H. Beech 7x10 Foot Low-Speed Wind Tunnel, Aerodynamic Laboratory, Wichita State University, Wichita KS, 1993.

10. Hoerner, S.F., Fluid Dynamic Drag, Hoerner Fluids Dynamics, Vancouver WA, 1965.

11. R. Lynette \& Associates, ESI-80 Rotor Performance and Reliability Enhancement Program, Final Report, Smith Wind Energy Corp., National Renewable Energy Laboratory Subcontract HC-211101, March 1993.

12. McCarty, J., PROP93 Users Guide Version 1.0, Alternative Energy Institute, West Texas A\&M University, Canyon TX, June 1993.

13. Advanced Wind Turbines, Inc., Draft Aerodynamic Brake System Specification, unreleased document, February 1996.

14. International Electrotechnical Commission, Wind Turbine Generator System-Part 1: Safety Requirements, International Standard 1400-1, December 1994.

15. Germanisher Lloyd, Rules and Regulations, IV-Non-Marine Technology, Part 1-Wind Energy, 1993.

16. Advanced Wind Turbines Inc., Loads Design Book, SSO03002, Revision A, March 1996. 
17. Spera, D.A., Esgar, J.B., Gougeon, M., and Zuteck, M.D., Structural Properties of Laminated Douglas Fir/Epoxy Composite Material, NASA Reference Publication 1236, DOE/NASA/2032076, 1990.

18. Ramsay, R.R., Hoffmann, G.M., and Gregorek, G.M., Effects of Grit Roughness and Pitch Oscillations on the S815 Airfoil, Midwest Research Institute Contract Number XF-11009-3, August 1994. 
APPENDIX A

\section{WSU Wind Tunnel Test Data}




\section{WSU Wind Tunnel Test Data}

The complete data set from the WSU/AWT/NREL aerodynamic brake wind tunnel test is available by request from NREL or WSU. The data are contained in an ASCII format text file, 'wsudata1.txt.' The data are arranged in columns, which contain run numbers, airfoil angle of attack, and aerodynamic coefficients, as shown in Table A-1. Aerodynamic coefficients are all corrected, and have been normalized as described in the report text. Table A-2 provides complete documentation of the configurations tested and the corresponding test run numbers. The test runs that indicate leading-edge grit roughness (LEGR) used \#60 lapidary grit with the standard NREL roughness template as described in Reference 18.

The majority of configurations tested are presented in graphical form in this appendix, with a summary given in Table A-3.

Table A-1. Configuration of Data File 'wsudata1.txt'

\begin{tabular}{cl}
\hline Column Number & \multicolumn{1}{c}{ Data Stored } \\
\hline 1 & Run number \\
2 & Corrected airfoil angle of attack (degrees) \\
3 & Airfoil lift coefficient, $C_{L}$ (wind axes) \\
4 & Airfoil drag coefficient, $C_{D}$ (wind axes) \\
5 & Airfoil 1/4-chord moment coefficient, $C_{M}$ (wind axes) \\
6 & Wind tunnel dynamic pressure, q (psf) \\
7 & Airfoil suction coefficient, $C_{S}$ (airfoil axes) \\
8 & Airfoil normal coefficient, $C_{N}$ (airfoil axes) \\
9 & Flap hinge moment coefficient \\
\hline
\end{tabular}


Table A-2. Run Log for WSU Aerodynamic Brake Wind Tunnel Test

\begin{tabular}{|c|c|c|c|c|c|c|c|}
\hline Run \# & $\operatorname{Re}$ & $\alpha$ & Configuration & Hinge & Device Chord & $\delta\left(^{\circ}\right)$ & Comments \\
\hline 5 & Low & 1 & $\mathrm{~S} 810$ & A-3 & 0.38 & 0 & Baseline airfoil, gaps sealed \\
\hline 6 & High & 2 & S810 & $A-3$ & 0.38 & 0 & « \\
\hline 7 & Low & 1 & S810 & A-3 & 0.38 & 0 & $\begin{array}{l}\text { Baseline airfoil, gap sealed } \\
\text { with } 51 \mathrm{~mm}(2 \mathrm{in}) \text { strip of } \\
\text { 3-M UV leading-edge tape }\end{array}$ \\
\hline 8 & High & 2 & S810 & A-3 & 0.38 & 0 & \\
\hline 9 & High & 2 & S810 & A-3 & 0.38 & 0 & Repeat of run $\# 8$ to $\alpha=24^{\circ}$ \\
\hline 10 & Low & 1 & S810 & A-3 & 0.38 & 0 & Repeat of run $\# 9$ to $\alpha=24^{\circ}$ \\
\hline 11 & Low & 1 & S810 & A-3 & 0.38 & 0 & Baseline airfoil, gaps open \\
\hline 12 & High & 2 & S810 & A-3 & 0.38 & 0 & “ \\
\hline 13 & Low & 1 & Spoiler-Flap & A-3 & 0.38 & 5 & $\delta$ sweep for hinge $A-3$ \\
\hline 14 & Low & 1 & Spoiler-Flap & A-3 & 0.38 & 10 & “ \\
\hline 15 & Low & 1 & Spoiler-Flap & A-3 & 0.38 & 30 & “ \\
\hline 16 & Low & 1 & Spoiler-Flap & A-3 & 0.38 & 60 & “ \\
\hline 17 & Low & 1 & Spoiler-Flap & A-3 & 0.38 & 90 & “ \\
\hline 18 & High & 2 & Spoiler-Flap & A-3 & 0.38 & 5 & Recheck for hinge $A-3$ \\
\hline 19 & High & 2 & Spoiler-Flap & A-3 & 0.38 & 10 & « \\
\hline 20 & Low & 1 & Spoiler-Flap & A-4 & 0.38 & 0 & $\delta$ sweep for hinge A-4 \\
\hline 21 & Low & 1 & Spoiler-Flap & A-4 & 0.38 & 5 & « \\
\hline 22 & Low & 1 & Spoiler-Flap & $A-4$ & 0.38 & 10 & “ \\
\hline 23 & Low & 1 & Spoiler-Flap & A-4 & 0.38 & 20 & “ \\
\hline 24 & Low & 1 & Spoiler-Flap & A-4 & 0.38 & 30 & “ \\
\hline 25 & Low & 1 & Spoiler-Flap & $A-4$ & 0.38 & 40 & “ \\
\hline 26 & Low & 1 & Spoiler-Flap & A-4 & 0.38 & 60 & “ \\
\hline 27 & Low & 1 & Spoiler-Flap & $A-4$ & 0.38 & 75 & “ \\
\hline 28 & Low & 1 & Spoiler-Flap & $A-4$ & 0.38 & 90 & “ \\
\hline 29 & Low & 1 & Spoiler-Flap & A-4 & 0.38 & 0 & Repeat of run $\# 20$ \\
\hline 30 & Low & 1 & Spoiler-Flap & A-4 & 0.38 & 5 & Repeat of run $\# 21$ \\
\hline 31 & High & 2 & Spoiler-Flap & $A-4$ & 0.38 & 0 & Recheck for hinge A-4 \\
\hline 32 & High & 2 & Spoiler-Flap & A-4 & 0.38 & 5 & « \\
\hline 33 & High & 2 & Spoiler-Flap & $A-4$ & 0.38 & 10 & “ \\
\hline 34 & Low & 1 & Spoiler-Flap & A-5 & 0.38 & 0 & $\delta$ sweep for hinge A-5 \\
\hline 35 & Low & 1 & Spoiler-Flap & A-5 & 0.38 & 5 & « \\
\hline 36 & Low & 1 & Spoiler-Flap & A- 5 & 0.38 & 10 & “ \\
\hline 37 & Low & 1 & Spoiler-Flap & A-5 & 0.38 & 20 & “ \\
\hline 38 & Low & 1 & Spoiler-Flap & A-5 & 0.38 & 30 & “ \\
\hline 39 & Low & 1 & Spoiler-Flap & A-5 & 0.38 & 40 & “ \\
\hline 40 & Low & 1 & Spoiler-Flap & A-5 & 0.38 & 60 & “ \\
\hline
\end{tabular}

Notes: $\alpha 1 \equiv-6^{\circ} \leq \alpha \leq 90^{\circ}$, with $3^{\circ}$ increments for $\alpha<30^{\circ}$ and $5^{\circ}$ increments for $\alpha>30^{\circ}$. $\alpha 2 \equiv-6^{\circ} \leq \alpha \leq 45^{\circ}$, with $3^{\circ}$ increments for $\alpha<30^{\circ}$ and $5^{\circ}$ increments for $\alpha>30^{\circ}$. $\alpha 3=-6^{\circ} \leq \alpha \leq 15^{\circ}$; with $3^{\circ}$ increments.

Re Low $\equiv$ Reynolds number nominally 1 million for entire run.

Re High $\equiv$ Reynolds number varies between 1.4 and 2.1 million throughout run, with wind tunnel operator maintaining maximum value without exceeding balance limits. 
Table A-2. Run Log for WSU Aerodynamic Brake Wind Tunnel Test (continued)

\begin{tabular}{|c|c|c|c|c|c|c|c|}
\hline Run \# & $\mathrm{Re}$ & $\alpha$ & Configuration & Hinge & Device Chord & $\delta\left({ }^{\circ}\right)$ & Comments \\
\hline 41 & Low & 1 & Spoiler-Flap & $A-5$ & 0.38 & 75 & $\delta$ sweep for hinge $A-5$ \\
\hline 42 & Low & 1 & Spoiler-Flap & $A-5$ & 0.38 & 90 & $\propto$ \\
\hline 43 & Low & 1 & Spoiler-Flap & $A-5$ & 0.38 & 0 & Repeat of run \#34 \\
\hline 44 & Low & 1 & Spoiler-Flap & A-5 & 0.38 & 10 & Repeat of run \#36 \\
\hline 45 & High & 2 & Spoiler-Flap & A-5 & 0.38 & 0 & Recheck of hinge A-5 \\
\hline 46 & High & 2 & Spoiler-Flap & A-5 & 0.38 & 10 & 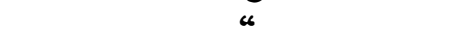 \\
\hline 47 & High & 2 & Spoiler-Flap & $A-5$ & 0.38 & 20 & “ \\
\hline 48 & High & 2 & Spoiler-Flap & $A-5$ & 0.38 & 60 & « \\
\hline 49 & Low & 1 & Plain Flap & $A-2$ & 0.38 & 0 & $\delta$ sweep for hinge $A-2$ \\
\hline 50 & Low & 1 & Plain Flap & A-2 & 0.38 & 15 & 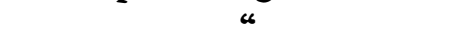 \\
\hline 51 & Low & 1 & Plain Flap & $A-2$ & 0.38 & 30 & 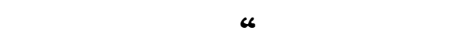 \\
\hline 52 & Low & 1 & Plain Flap & A-2 & 0.38 & 45 & “ \\
\hline 53 & Low & 1 & Plain Flap & A-2 & 0.38 & 60 & “ \\
\hline 54 & Low & 1 & Plain Flap & $A-2$ & 0.38 & 90 & 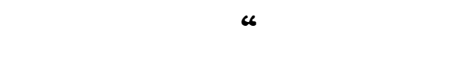 \\
\hline 55 & Low & 1 & Plain Flap & B-1 & 0.38 & 0 & $\delta$ sweep for hinge B-1 \\
\hline 56 & Low & 1 & Plain Flap & B-1 & 0.38 & 15 & 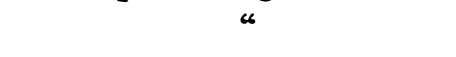 \\
\hline 57 & Low & 1 & Plain Flap & B-1 & 0.38 & 30 & “ \\
\hline 58 & Low & 1 & Plain Flap & B-1 & 0.38 & 45 & “ \\
\hline 59 & Low & 1 & Plain Flap & B-1 & 0.38 & 60 & “ \\
\hline 60 & Low & 1 & Plain Flap & B-1 & 0.38 & 75 & “ \\
\hline 61 & Low & 1 & Plain Flap & B-1 & 0.38 & 90 & « \\
\hline 62 & Low & 1 & Vented Flap & $\mathrm{C}-2$ & 0.38 & 0 & $\delta$ sweep for hinge B-1 \\
\hline 63 & Low & 1 & Vented Flap & $\mathrm{C}-2$ & 0.38 & 15 & « \\
\hline 64 & Low & 1 & Vented Flap & $\mathrm{C}-2$ & 0.38 & 30 & “ \\
\hline 65 & Low & 1 & Vented Flap & $\mathrm{C}-2$ & 0.38 & 45 & « \\
\hline 66 & Low & 1 & S810 & A-1 & 0.45 & 0 & Baseline airfoil, gaps sealed \\
\hline 67 & High & 2 & S810 & A-1 & 0.45 & 0 & « \\
\hline 68 & Low & 1 & S810 & $A-1$ & 0.45 & 0 & Baseline airfoil, gaps open \\
\hline 69 & High & 2 & S810 & $A-1$ & 0.45 & 0 & c \\
\hline 70 & Low & 1 & Plain Flap & A-1 & 0.45 & 15 & $\delta$ sweep for hinge $A-1$ \\
\hline 71 & Low & 1 & Plain Flap & $A-1$ & 0.45 & 30 & 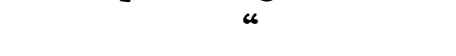 \\
\hline 72 & Low & 1 & Plain Flap & A-1 & 0.45 & 45 & “ \\
\hline 73 & Low & 1 & Plain Flap & $A-1$ & 0.45 & 60 & “ \\
\hline 74 & Low & 1 & Plain Flap & $A-1$ & 0.45 & 75 & “ \\
\hline 75 & Low & 1 & Plain Flap & A-1 & 0.45 & 90 & 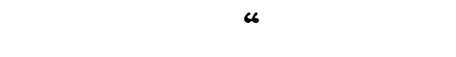 \\
\hline 76 & Low & 1 & Spoiler-Flap & $A-2$ & 0.45 & 0 & $\delta$ sweep for hinge $A-2$ \\
\hline 77 & Low & 1 & Spoiler-Flap & $A-2$ & 0.45 & 15 & 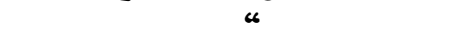 \\
\hline 78 & Low & 1 & Spoiler-Flap & A-2 & 0.45 & 30 & 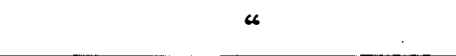 \\
\hline
\end{tabular}

Notes: $\alpha 1 \equiv-6^{\circ} \leq \alpha \leq 90^{\circ}$, with $3^{\circ}$ increments for $\alpha<30^{\circ}$ and $5^{\circ}$ increments for $\alpha>30^{\circ}$. $\alpha 2 \equiv-6^{\circ} \leq \alpha \leq 45^{\circ}$, with $3^{\circ}$ increments for $\alpha<30^{\circ}$ and $5^{\circ}$ increments for $\alpha>30^{\circ}$. $\alpha 3 \equiv-6^{\circ} \leq \alpha \leq 15^{\circ}$, with $3^{\circ}$ increments.

Re Low $\equiv$ Reynolds number nominally 1 million for entire run.

Re High $\equiv$ Reynolds number varies between 1.4 and 2.1 million throughout run, with wind tunnel operator maintaining maximum value without exceeding balance limits. 
Table A-2. Run Log for WSU Aerodynamic Brake Wind Tunnel Test (continued)

\begin{tabular}{|c|c|c|c|c|c|c|c|}
\hline Run \# & $\mathrm{Re}$ & $\alpha$ & Configuration & Hinge & Device Chord & $\delta\left(^{\circ}\right)$ & Comments \\
\hline 79 & Low & 1 & Spoiler-Flap & $A-2$ & 0.45 & 45 & $\delta$ sweep for hinge $A-2$ \\
\hline 80 & Low & 1 & Spoiler-Flap & A-2 & 0.45 & 60 & 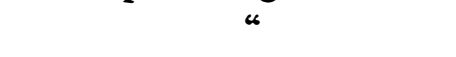 \\
\hline 81 & Low & 1 & Spoiler-Flap & $A-2$ & 0.45 & 75 & 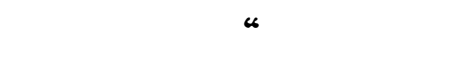 \\
\hline 82 & Low & 1 & Spoiler-Flap & $A-2$ & 0.45 & 90 & « \\
\hline 83 & Low & 1 & Spoiler-Flap & $A-3$ & 0.45 & 0 & $\delta$ sweep for hinge $A-3$ \\
\hline 84 & Low & 1 & Spoiler-Flap & $A-3$ & 0.45 & 15 & $\omega$ \\
\hline 85 & Low & 1 & Spoiler-Flap & $A-3$ & 0.45 & 30 & “ \\
\hline 86 & Low & 1 & Spoiler-Flap & $A-3$ & 0.45 & 45 & “ \\
\hline 87 & Low & 1 & Spoiler-Flap & A-3 & 0.45 & 60 & “ \\
\hline 88 & Low & 1 & Spoiler-Flap & A-3 & 0.45 & 75 & “ \\
\hline 89 & Low & 1 & Spoiler-Flap & $A-3$ & 0.45 & 90 & « \\
\hline 90 & Low & 1 & Spoiler-Flap & A-4 & 0.45 & 0 & $\delta$ sweep for hinge $A-4$ \\
\hline 91 & Low & 1 & Spoiler-Flap & $A-4$ & 0.45 & 15 & 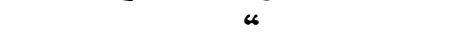 \\
\hline 92 & Low & 1 & Spoiler-Flap & $A-4$ & 0.45 & 30 & « \\
\hline 93 & Low & 1 & Spoiler-Flap & $A-4$ & 0.45 & 45 & “ \\
\hline 94 & Low & 1 & Spoiler-Flap & $A-4$ & 0.45 & 60 & 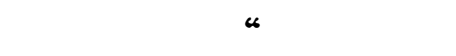 \\
\hline 95 & Low & 1 & Spoiler-Flap & A-4 & 0.45 & 75 & “ \\
\hline 96 & Low & 1 & Spoiler-Flap & $A-4$ & 0.45 & 90 & “ \\
\hline 97 & Low & 1 & Spoiler-Flap & B-3 & 0.45 & 0 & $\delta$ sweep for hinge B-3 \\
\hline 98 & Low & 1 & Spoiler-Flap & B-3 & 0.45 & 15 & $u$ \\
\hline 99 & Low & 1 & Spoiler-Flap & B-3 & 0.45 & 30 & “ \\
\hline 100 & Low & 1 & Spoiler-Flap & B-3 & 0.45 & 45 & “ \\
\hline 101 & Low & 1 & Spoiler-Flap & B-3 & 0.45 & 60 & “ \\
\hline 102 & Low & 1 & Spoiler-Flap & B-3 & 0.45 & 75 & « \\
\hline 103 & Low & 1 & Spoiler-Flap & B-3 & 0.45 & 90 & “ \\
\hline 104. & Low & 1 & Spoiler-Flap & B-5 & 0.45 & 0 & $\delta$ sweep for hinge B-5 \\
\hline $105^{\circ}$ & Low & 1 & Spoiler-Flap & B-5 & 0.45 & 15 & $\ll$ \\
\hline 106 & Low & 1 & Spoiler-Flap & B-5 & 0.45 & 30 & “ \\
\hline 107 & Low & 1 & Spoiler-Flap & B-5 & 0.45 & 45 & « \\
\hline 108 & Low & 1 & Spoiler-Flap & B-5 & 0.45 & 60 & “ \\
\hline 109 & Low & 1 & Spoiler-Flap & B-5 & 0.45 & 75 & 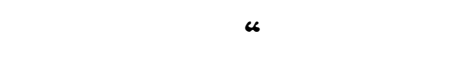 \\
\hline 110 & Low & 1 & Vented Flap & $\mathrm{C}-1$ & 0.45 & 0 & $\delta$ sweep for hinge $C-1$ \\
\hline 111 & Low & 1 & Vented Flap & $\mathrm{C}-1$ & 0.45 & 15 & 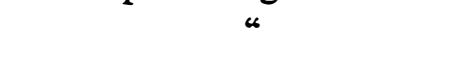 \\
\hline 112 & Low & 1 & Vented Flap & $\mathrm{C}-1$ & 0.45 & 30 & “ \\
\hline 113 & Low & 1 & Vented Flap & $\mathrm{C}-1$ & 0.45 & 45 & “ \\
\hline 114 & Low & 1 & Vented Flap & $\mathrm{C}-1$ & 0.45 & 60 & 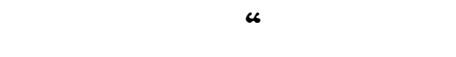 \\
\hline 115 & Low & 1 & Vented Flap & C-1 & 0.45 & 75 & “ \\
\hline 116 & Low & 1 & Vented Flap & $\mathrm{C}-1$ & 0.45 & 90 & $\omega$ \\
\hline Notes: & 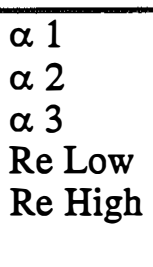 & $\begin{array}{l}\equiv \\
\equiv \\
\equiv \\
\equiv \\
\equiv\end{array}$ & $\begin{array}{l}-6^{\circ} \leq \alpha \leq 90^{\circ}, \text { " } \\
-6^{\circ} \leq \alpha \leq 45^{\circ}, \text { n } \\
-6^{\circ} \leq \alpha \leq 15^{\circ}, \text { n } \\
\text { Reynolds numbe } \\
\text { Reynolds numbe } \\
\text { tunnel operator }\end{array}$ & $\begin{array}{l}3^{\circ} \text { inc } \\
3^{\circ} \text { inc } \\
3^{\circ} \text { inc } \\
\text { lomina } \\
\text { aries }\end{array}$ & $\begin{array}{l}\text { ments for } \alpha \\
\text { ments for } \alpha \\
1 \text { million for } \\
\text { meen } 1.4 \text { and }\end{array}$ & 1 mill & $\begin{array}{l}5^{\circ} \text { increments for } \alpha>30^{\circ} \text {. } \\
5^{\circ} \text { increments for } \alpha>30^{\circ} \text {. } \\
\text { in throughout run, with wind } \\
\text { exceeding balance limits. }\end{array}$ \\
\hline
\end{tabular}


Table A-2. Run Log for WSU Aerodynamic Brake Wind Tunnel Test (continued)

\begin{tabular}{|c|c|c|c|c|c|c|c|}
\hline Run \# & $\operatorname{Re}$ & $\alpha$ & Configuration & Hinge & Device Chord & $\delta\left(^{\circ}\right)$ & Comments \\
\hline 117 & Low & 1 & Spoiler-Flap & $\mathrm{C}-3$ & 0.45 & 0 & $\delta$ sweep for hinge $C-3$ \\
\hline 118 & Low & 1 & Spoiler-Flap & C-3 & 0.45 & 15 & u \\
\hline 119 & Low & 1 & Spoiler-Flap & $\mathrm{C}-3$ & 0.45 & 30 & “ \\
\hline 120 & Low & 1 & Spoiler-Flap & C-3 & 0.45 & 45 & “ \\
\hline 121 & Low & 1 & Spoiler-Flap & $\mathrm{C}-3$ & 0.45 & 60 & “ \\
\hline 122 & Low & 1 & Spoiler-Flap & $\mathrm{C}-3$ & 0.45 & 85 & “ \\
\hline 123 & Low & 1 & Spoiler-Flap & C-5 & 0.45 & 0 & $\delta$ sweep for hinge $C-5$ \\
\hline 124 & Low & 1 & Spoiler-Flap & C-5 & 0.45 & 15 & 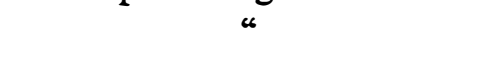 \\
\hline 125 & Low & 1 & Spoiler-Flap & C-5 & 0.45 & 30 & “ \\
\hline 126 & Low & 1 & Spoiler-Flap & C-5 & 0.45 & 45 & “ \\
\hline 127 & Low & 1 & Spoiler-Flap & C-5 & 0.45 & 60 & u \\
\hline 128 & Low & 2 & Partial Span & $A-4$ & 0.45 & 0 & $\begin{array}{l}\text { Full-span spoiler ( } 90^{\circ} \text { upper) } \\
\text { and split-flap }\left(90^{\circ} \text { lower }\right)\end{array}$ \\
\hline 129 & Low & 2 & Partial Span & $A-4$ & 0.45 & 0 & Repeat run \#128 \\
\hline 130 & Low & 2 & Partial Span & $A-4$ & 0.45 & 0 & $\begin{array}{l}\text { 1/2-span spoiler ( } 90^{\circ} \text { upper) } \\
\text { and split-flap ( } 90^{\circ} \text { lower) }\end{array}$ \\
\hline 131 & Low & 2 & Partial Span & $A-4$ & 0.45 & 0 & re-reduction of run $\# 103$ data \\
\hline 132 & Low & 2 & Partial Span & $A-4$ & 0.45 & 0 & $\begin{array}{l}\text { 1/3-span spoiler ( } 90^{\circ} \text { upper) } \\
\text { and split-flap }\left(90^{\circ} \text { lower }\right)\end{array}$ \\
\hline 133 & Low & 2 & Partial Span & $A-4$ & 0.45 & 0 & $\begin{array}{l}\text { Full-span spoiler }\left(90^{\circ} \text { upper }\right) \\
\text { and split-flap }\left(45^{\circ} \text { lower }\right)\end{array}$ \\
\hline 134 & Low & 2 & Partial Span & $A-4$ & 0.45 & 0 & $\begin{array}{l}\text { 1/2-span spoiler ( } 90^{\circ} \text { upper) } \\
\text { and split-flap ( } 45^{\circ} \text { lower) }\end{array}$ \\
\hline 135 & Low & 2 & Partial Span & $A-4$ & 0.45 & 0 & $\begin{array}{l}1 / 3 \text {-span spoiler ( } 90^{\circ} \text { upper) } \\
\text { and split-flap ( } 45^{\circ} \text { lower) }\end{array}$ \\
\hline 136 & Low & 2 & Partial Span & $A-4$ & 0.45 & 0 & $\begin{array}{l}\text { 1/3-span split-flap only } \\
\left(45^{\circ} \text { down) }\right.\end{array}$ \\
\hline 137 & Low & 2 & Partial Span & $A-4$ & 0.45 & 0 & $\begin{array}{l}\text { 1/2-span split-flap only } \\
\left(45^{\circ} \text { lower }\right)\end{array}$ \\
\hline 138 & Low & 2 & Partial Span & $A-4$ & 0.45 & 0 & $\begin{array}{l}\text { Full-span split-flap only } \\
\left(45^{\circ} \text { lower }\right)\end{array}$ \\
\hline 139 & Low & 2 & Partial Span & $A-4$ & 0.45 & 0 & $\begin{array}{l}\text { Full-span split-flap only } \\
\left(45^{\circ} \text { lower }\right)\end{array}$ \\
\hline 140 & Low & 2 & Partial Span & $A-4$ & 0.45 & 0 & $\begin{array}{l}\text { 1/2-span split-flap only } \\
\left(45^{\circ} \text { lower }\right)\end{array}$ \\
\hline 141 & Low & 2 & Partial Span & $A-4$ & 0.45 & 0 & $\begin{array}{l}\text { 1/3-span split-flap only } \\
\left(45^{\circ} \text { lower }\right)\end{array}$ \\
\hline 142 & Low & 2 & Partial Span & $A-4$ & 0.45 & 0 & $\begin{array}{l}\text { 1/3-span spoiler only } \\
\text { (90 upper) }\end{array}$ \\
\hline 143 & Low & 2 & Partial Span & $A-4$ & 0.45 & 0 & $\begin{array}{l}\text { 1/2-span spoiler only } \\
\text { (90 upper) }\end{array}$ \\
\hline
\end{tabular}

Notes: $\alpha 1=-6^{\circ} \leq \alpha \leq 90^{\circ}$, with $3^{\circ}$ increments for $\alpha<30^{\circ}$ and $5^{\circ}$ increments for $\alpha>30^{\circ}$. $\alpha 2 \quad=-6^{\circ} \leq \alpha \leq 45^{\circ}$, with $3^{\circ}$ increments for $\alpha<30^{\circ}$ and $5^{\circ}$ increments for $\alpha>30^{\circ}$. $\alpha 3=-6^{\circ} \leq \alpha \leq 15^{\circ}$, with $3^{\circ}$ increments.

Re Low $\equiv$ Reynolds number nominally 1 million for entire run.

Re High $=$ Reynolds number varies between 1.4 and 2.1 million throughout run, with wind tunnel operator maintaining maximum value without exceeding balance limits. 
Table A-2. Run Log for WSU Aerodynamic Brake Wind Tunnel Test (continued)

\begin{tabular}{|c|c|c|c|c|c|c|c|}
\hline Run \# & $\mathrm{Re}$ & $\alpha$ & Configuration & Hinge & Device Chord & $\delta\left(^{\circ}\right)$ & Comments \\
\hline 144 & Low & 2 & Partial Span & $A-4$ & 0.45 & 0 & $\begin{array}{l}\text { Full-span spoiler only } \\
\text { (90 upper) }\end{array}$ \\
\hline 145 & Low & 1 & Spoiler-Flap & A-4 & 0.45 & 0 & Repeat of run \#90 \\
\hline 146 & High & 2 & Spoiler-Flap & $A-4$ & 0.45 & 0 & Hinge $A-4$ at high $R e$ \\
\hline 147 & Low & 1 & Spoiler-Flap & A-4 & 0.45 & 5 & Hinge A-4 at small $\delta$ \\
\hline 148 & Low & 1 & Spoiler-Flap & A-4 & 0.45 & 10 & “ \\
\hline 149 & Low & 1 & Spoiler-Flap & A-4 & 0.45 & 15 & Repeat of run \#91 \\
\hline 150 & High & 2 & Spoiler-Flap & A-4 & 0.45 & 15 & Hinge $A-4$ at high $\operatorname{Re}$ \\
\hline 151 & High & 2 & Spoiler-Flap & A-4 & 0.45 & 30 & « \\
\hline 152 & High & 2 & Spoiler-Flap & A-4 & 0.45 & 45 & “ \\
\hline 153 & High & 2 & Spoiler-Flap & A-4 & 0.45 & 60 & “ \\
\hline 154 & High & 2 & Spoiler-Flap & A-4 & 0.45 & 75 & “ \\
\hline 155 & Low & 2 & Spoiler-Flap & A-4 & 0.45 & 30 & $\begin{array}{l}\text { Hinge A-4 with rounded } \\
\text { cove insert }\end{array}$ \\
\hline 156 & Low & 2 & Spoiler-Flap & $A-4$ & 0.45 & 23 & $\begin{array}{l}\text { Hinge A-4 with rounded } \\
\text { cove insert }\end{array}$ \\
\hline 157 & Low & 3 & S810 & A-4 & 0.45 & 0 & $\begin{array}{l}\text { Repeat measurement of low } \\
\text { Re drag bucket with gap } \\
\text { sealed (run \# } 66 \text { at low } \alpha \text { ) }\end{array}$ \\
\hline 158 & High & 3 & S810 & $A-4$ & 0.45 & 0 & $\begin{array}{l}\text { Repeat measurement of high } \\
\text { Re drag bucket with gap } \\
\text { sealed (run \# } 67 \text { at low } \alpha \text { ) }\end{array}$ \\
\hline 159 & Low & 3 & S810 & A-4 & 0.45 & 0 & $\begin{array}{l}\text { Gap-sealed, low Re drag } \\
\text { bucket with } 51 \mathrm{~mm}(2 \mathrm{in}) \\
\text { strip of } 0.15 \mathrm{~mm} \text { ( } 6 \mathrm{mil}) \\
\text { thick leading-edge tape }\end{array}$ \\
\hline 160 & Low & 3 & S810 & A-4 & 0.45 & 0 & $\begin{array}{l}\text { Gap-sealed, high Re drag } \\
\text { bucket with } 51 \mathrm{~mm}(2 \mathrm{in}) \\
\text { strip of } 0.15 \mathrm{~mm}(6 \mathrm{mil}) \\
\text { thick leading-edge tape }\end{array}$ \\
\hline 161 & Low & 2 & Spoiler-Flap & $A-4$ & 0.45 & 0 & $\begin{array}{l}\text { Hinge A-4 with leading-edge } \\
\text { grain roughness (LEGR) }\end{array}$ \\
\hline 162 & High & 2 & Spoiler-Flap & A-4 & 0.45 & 0 & c \\
\hline 163 & Low & 2 & Spoiler-Flap & $A-4$ & 0.45 & 15 & “ \\
\hline 164 & High & 2 & Spoiler-Flap & $A-4$ & 0.45 & 15 & “ \\
\hline 165 & Low & 2 & Spoiler-Flap & $A-4$ & 0.45 & 30 & “ \\
\hline 166 & High & 2 & Spoiler-Flap & $A-4$ & 0.45 & 30 & “ \\
\hline 167 & Low & 2 & Spoiler-Flap & $A-4$ & 0.45 & 45 & “ \\
\hline 168 & Low & 2 & Spoiler-Flap & $A-4$ & 0.45 & 60 & “ \\
\hline 169 & Low & 2 & Spoiler-Flap & A-4 & 0.45 & 75 & “ \\
\hline 170 & Low & 2 & Spoiler-Flap & A-4 & 0.45 & 90 & “ \\
\hline
\end{tabular}

Notes: $\alpha 1 \equiv-6^{\circ} \leq \alpha \leq 90^{\circ}$, with $3^{\circ}$ increments for $\alpha<30^{\circ}$ and $5^{\circ}$ increments for $\alpha>30^{\circ}$. $\alpha 2 \equiv-6^{\circ} \leq \alpha \leq 45^{\circ}$, with $3^{\circ}$ increments for $\alpha<30^{\circ}$ and $5^{\circ}$ increments for $\alpha>30^{\circ}$. $\alpha 3 \equiv-6^{\circ} \leq \alpha \leq 15^{\circ}$, with $3^{\circ}$ increments.

Re Low $\equiv$ Reynolds number nominally 1 million for entire run.

Re High $\equiv$ Reynolds number varies between 1.4 and 2.1 million throughout run, with wind tunnel operator maintaining maximum value without exceeding balance limits. 
Table A-3. Summary of Appendix A Graphical Data

\begin{tabular}{|c|c|c|}
\hline $\begin{array}{l}\text { Figure } \\
\text { Number }\end{array}$ & Title & $\begin{array}{l}\text { Run Numbers } \\
\text { Plotted }\end{array}$ \\
\hline A-1 & $\delta$-Sweep for $38 \%$ Cord Plain Flap, Hinge A-2 & $49,50,51,52,53,54$ \\
\hline A-2 & $\delta$-Sweep for $38 \%$ Cord Spoiler-Flap, Hinge A-3 & $11,13,14,15,16,17$ \\
\hline A-3 & $\delta$-Sweep for $38 \%$ Cord Spoiler-Flap, Hinge A-4 & $20,21,22,23,24,25,26,27,28$ \\
\hline A-4 & $\delta$-Sweep for $38 \%$ Cord Spoiler-Flap, Hinge A-5 & $34,35,36,37,38,39,40,41,42$ \\
\hline A-5 & $\delta$-Sweep for $38 \%$ Cord Plain Flap, Hinge B-1 & $55,56,57,58,59,60,61$ \\
\hline A-6 & $\delta$-Sweep for $38 \%$ Cord Vented Flap, Hinge C-2 & $62,63,64,65$ \\
\hline A-7 & $\delta$-Sweep for $45 \%$ Cord Plain Flap, Hinge A-1 & $70,71,72,73,74,75$ \\
\hline A-8 & $\delta$-Sweep for $45 \%$ Cord Spoiler-Flap, Hinge A-2 & $76,77,78,79,80,81,82$ \\
\hline A-9 & $\delta$-Sweep for $45 \%$ Cord Spoiler-Flap, Hinge A-3 & $83,84,85,86,87,88,89$ \\
\hline A-10 & $\delta$-Sweep for $45 \%$ Cord Spoiler-Flap, Hinge A-4 & $90,147,148,91,92,93,94,95$ \\
\hline A-11 & $\delta$-Sweep for $45 \%$ Cord Spoiler-Flap, Hinge B-3 & $97,98,99,100,101,102,103$ \\
\hline A-12 & $\delta$-Sweep for $45 \%$ Cord Spoiler-Flap, Hinge B-5 & $104,105,106,107,108,109$ \\
\hline A-13 & $\delta$-Sweep for $45 \%$ Cord Vented Flap, Hinge C-1 & $110,111,112,113,114,115,116$ \\
\hline A-14 & $\delta$-Sweep for $45 \%$ Cord Spoiler-Flap, Hinge C-3 & $117,118,119,120,121,122$ \\
\hline$A-15$ & $\delta$-Sweep for $45 \%$ Cord Spoiler-Flap, Hinge C-5 & $123,124,125,126,127$ \\
\hline A-16 & Repeatability Check on Spoiler-Flap & $90,91,145,149$ \\
\hline A-17 & Reynolds Number Effect on Spoiler-Flap & $90,146,92,151,94,153$ \\
\hline A-18 & Effect of Leading-Edge Roughness on Spoiler-Flap & $90,161,92,165,94,168$ \\
\hline A-19 & Effect of Gap Seal on 38\% Chord Device & $5,6,11,12$ \\
\hline A-20 & Effect of Gap Seal on $45 \%$ Chord Device & $66,67,68,69$ \\
\hline$A-21$ & Effect of Leading-Edge Tape on Clean S810 & $66,67,7,8,159,160$ \\
\hline A-22 & Effect of Hinge Location on Device at $\delta=45^{\circ}$ & $72,79,86,93,100,113$ \\
\hline$A-23$ & Effect of Hinge Location on Device at $\delta=75^{\circ}$ & $74,81,88,95,102,115$ \\
\hline
\end{tabular}




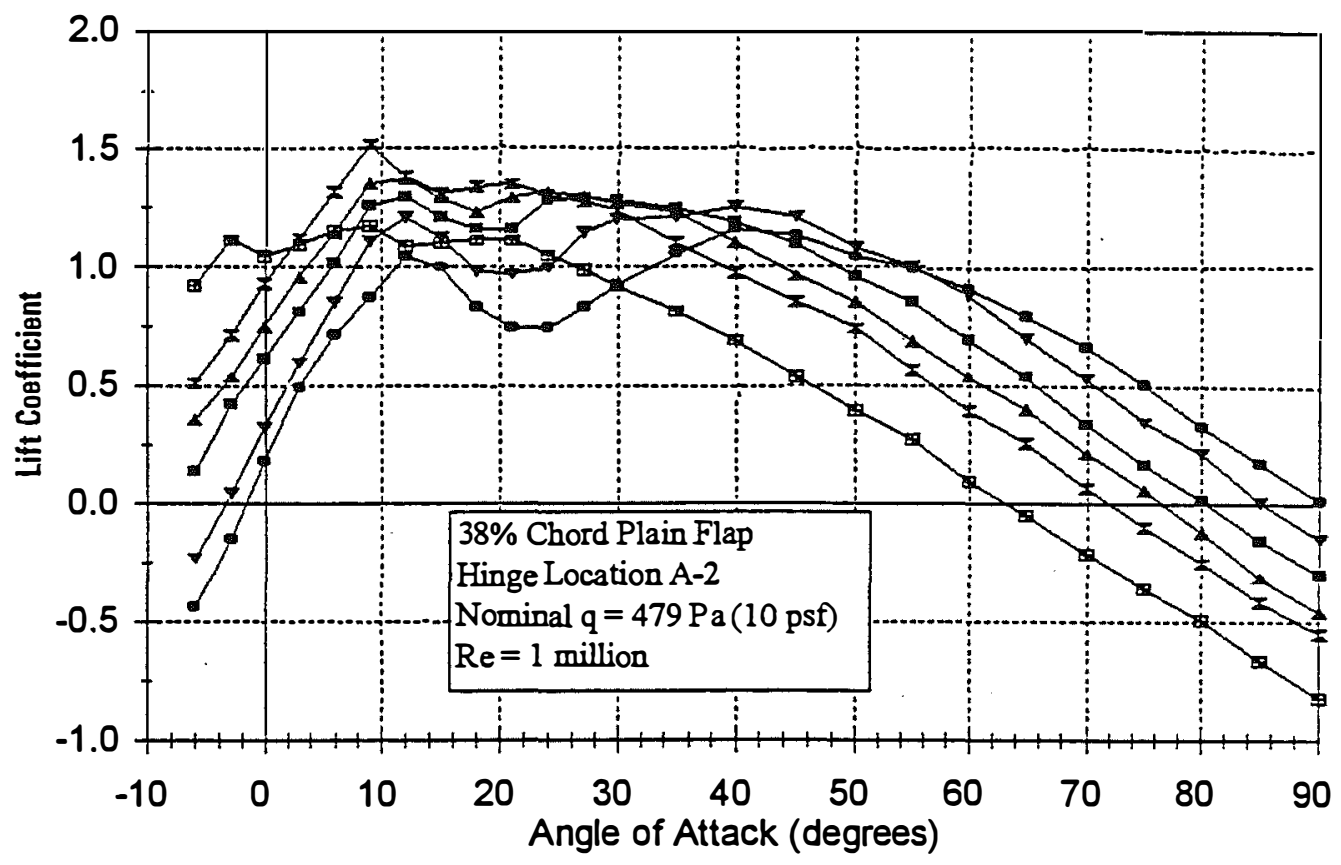

$$
\begin{aligned}
& - \text { Delta }=0 \rightarrow \text { Delta }=15 \rightarrow \text { Delta }=30 \\
& - \text { Delta }=45 \rightarrow \text { Delta }=60 \rightarrow \text { Delta }=90
\end{aligned}
$$

Figure A-1a. Lift Data, $\delta$ Sweep for $38 \%$ Chord Plain Flap, Hinge A-2

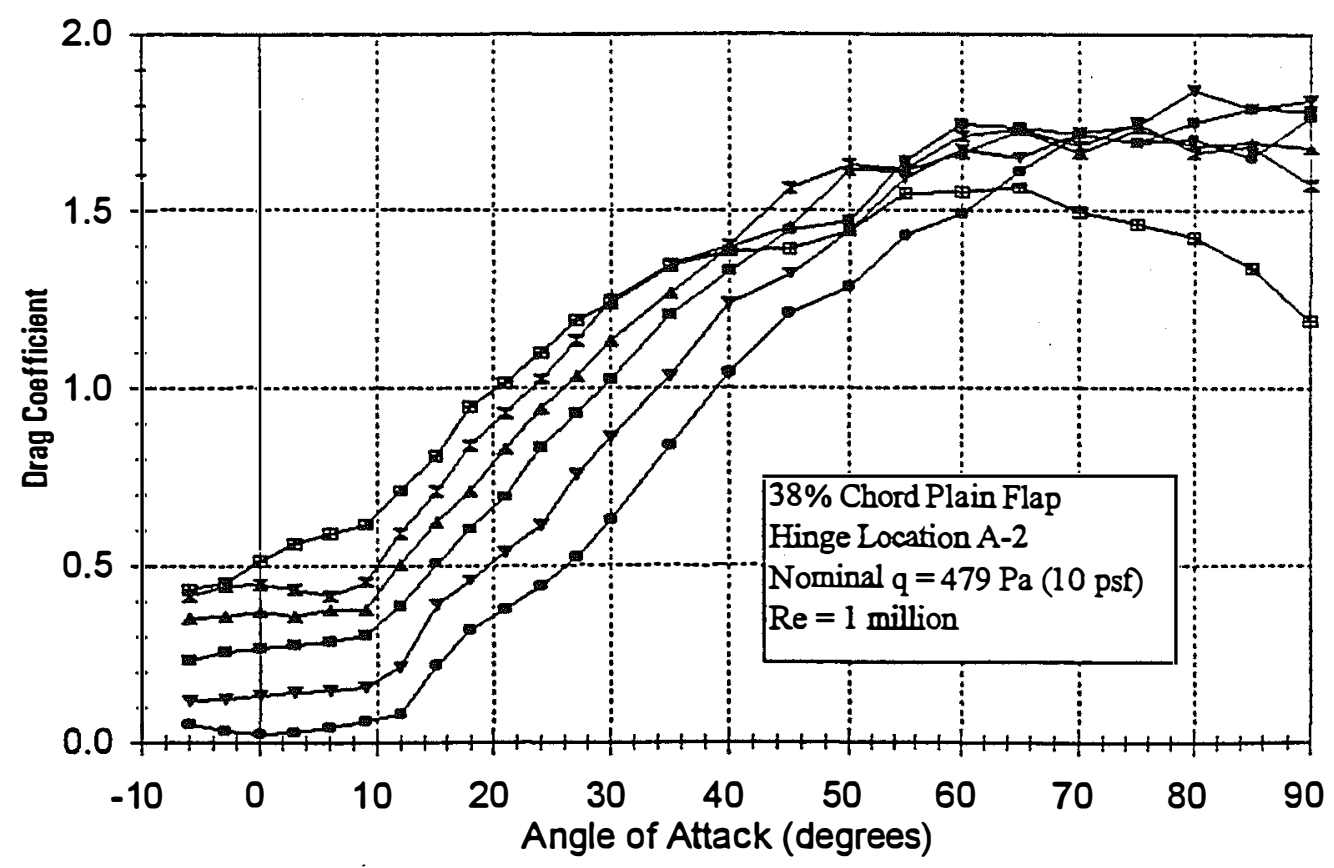

$$
\begin{aligned}
& \rightarrow \text { Delta }=0 \rightarrow \text { Delta }=15 \rightarrow \text { Delta }=30 \\
& \rightarrow \text { Delta }=45 \rightarrow \text { Delta }=60 \rightarrow \text { Delta }=90
\end{aligned}
$$

Figure A-1b. Drag Data, $\delta$ Sweep for $38 \%$ Chord Plain Flap, Hinge A-2 


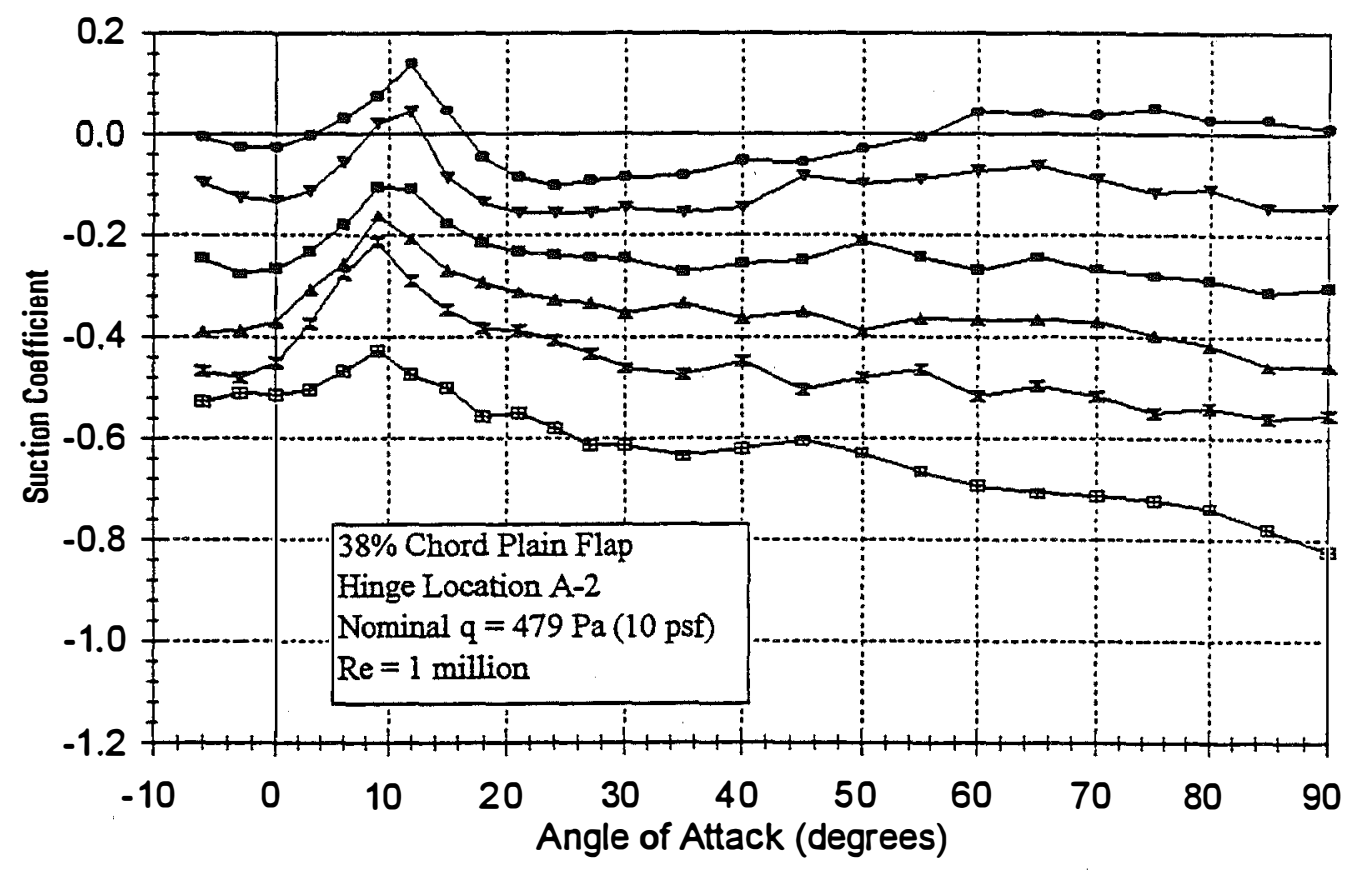

$$
\begin{aligned}
& \rightarrow \text { Delta }=0 \rightarrow \text { Delta }=15 \rightarrow \text { Delta }=30 \\
& - \text { Delta }=45 \rightarrow \text { Delta }=60 \rightarrow \text { Delta }=90
\end{aligned}
$$

Figure A-1c. Suction Data, $\delta$-Sweep for $38 \%$ Chord Plain Flap, Hinge A-2

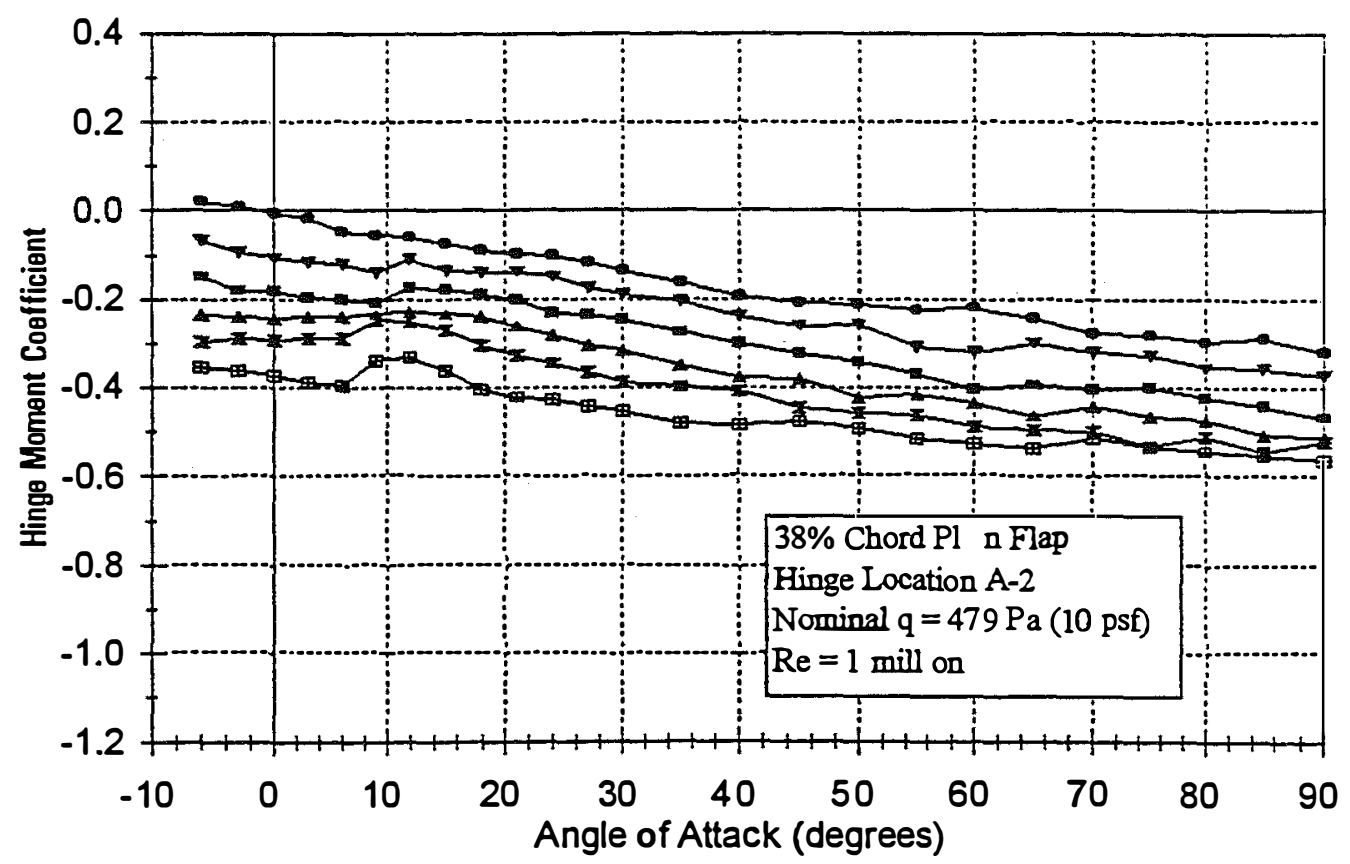

$$
\begin{aligned}
& \rightarrow \text { Delta }=0 \rightarrow \text { Delta }=15 \rightarrow \text { Delta }=30 \\
& - \text { Delta }=45 \rightarrow \text { Delta }=60 \rightarrow \text { Delta }=90
\end{aligned}
$$

Figure A-1d. Hinge Moment Data, $\delta$ Sweep for $38 \%$ Chord Plain Flap, Hinge A-2 


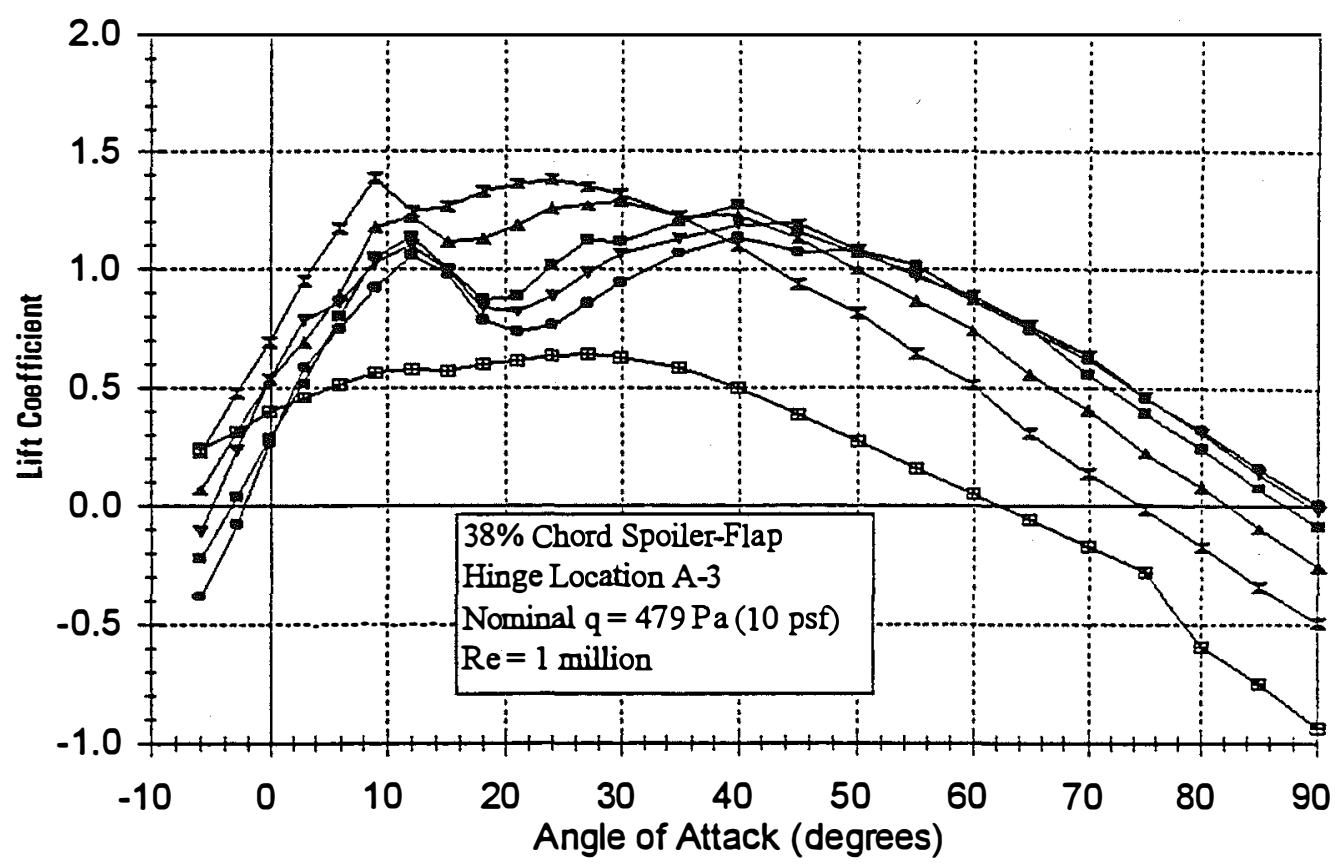

$$
\begin{aligned}
& \longrightarrow \text { Delta }=0 \rightarrow \text { Delta }=5 \rightarrow \text { Delta }=10 \\
& - \text { Delta }=30 \rightarrow-\text { Delta }=60 \rightarrow \text { Delta }=90
\end{aligned}
$$

Figure A-2a. Lift Data, $\delta$-Sweep for $38 \%$ Chord Spoiler-Flap, Hinge A-3

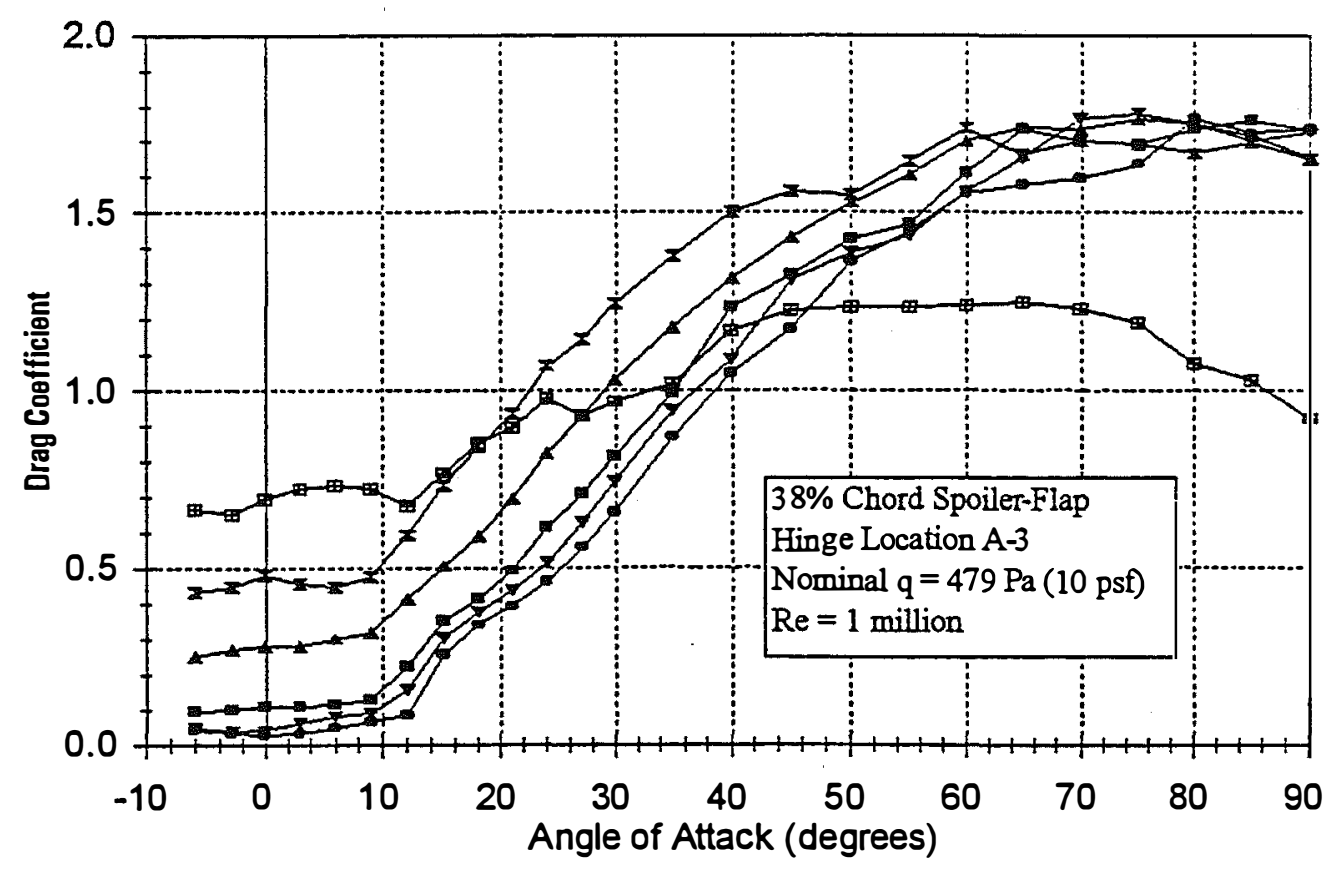

$$
\begin{aligned}
& \longrightarrow \text { Delta }=0 \rightarrow \text { Delta }=5 \rightarrow \text { Delta }=10 \\
& - \text { Delta }=30 \rightarrow-\text { Delta }=60 \rightarrow \text { Delta }=90
\end{aligned}
$$

Figure A-2b. Drag Data, $\delta$-Sweep for $38 \%$ Chord Spoiler-Flap, Hinge A-3 


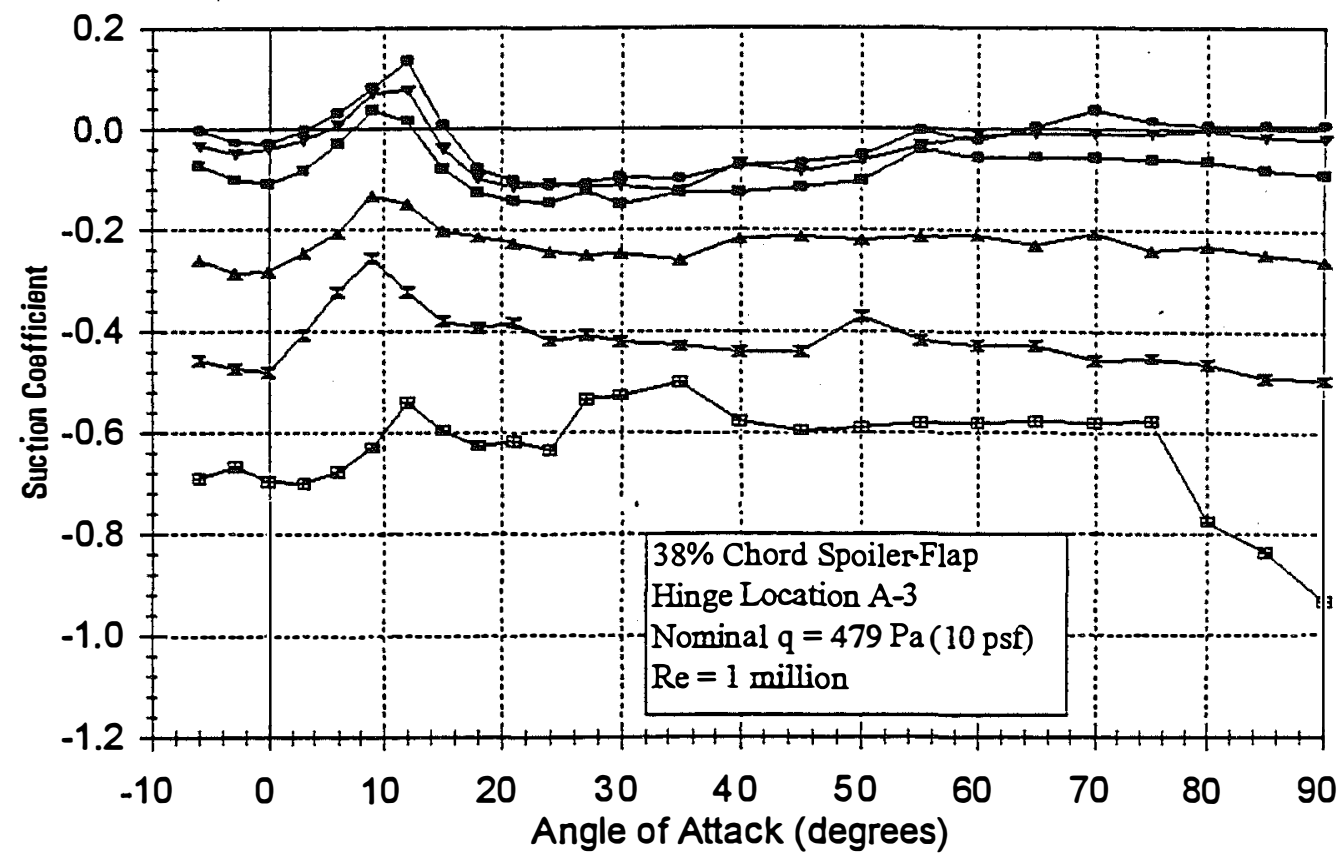

$$
\begin{aligned}
& \rightarrow \text { Delta }=0 \rightarrow \text { Delta }=5 \rightarrow \text { Delta }=10 \\
& - \text { Delta }=30 \rightarrow \text { Delta }=60 \rightarrow \text { Delta }=90
\end{aligned}
$$

Figure A-2c. Suction Data, $\delta$ Sweep for $38 \%$ Chord Spoiler-Flap, Hinge A-3

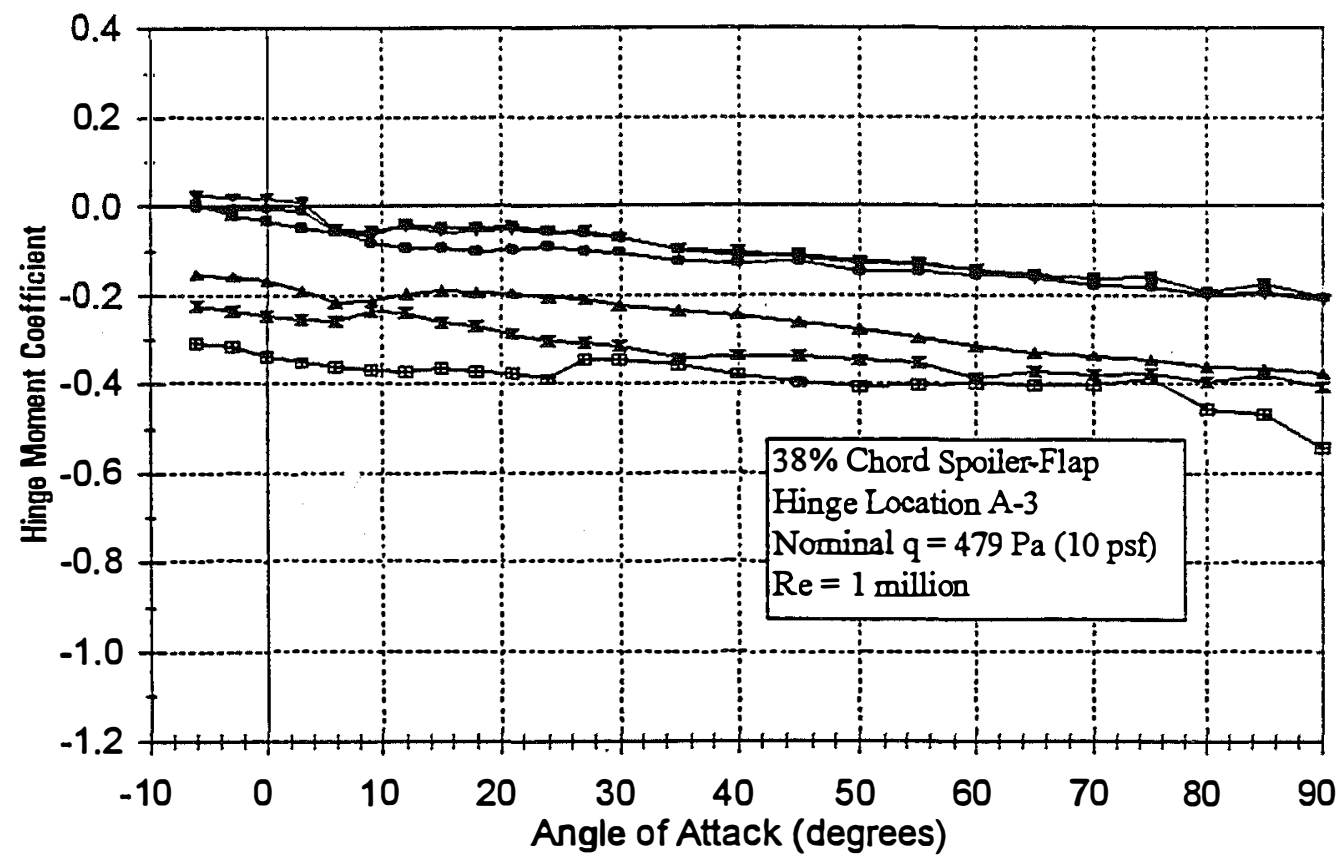

$$
\begin{aligned}
& \text { Delta }=0 \rightarrow \text { Delta }=5 \rightarrow \text { Delta }=10 \\
& \text { Delta }=30 \rightarrow \text { Delta }=60 \rightarrow \text { Delta }=90
\end{aligned}
$$

Figure A-2d. Hinge Moment Data, $\delta$-Sweep for $38 \%$ Chord Spoiler-Flap, Hinge A-3 


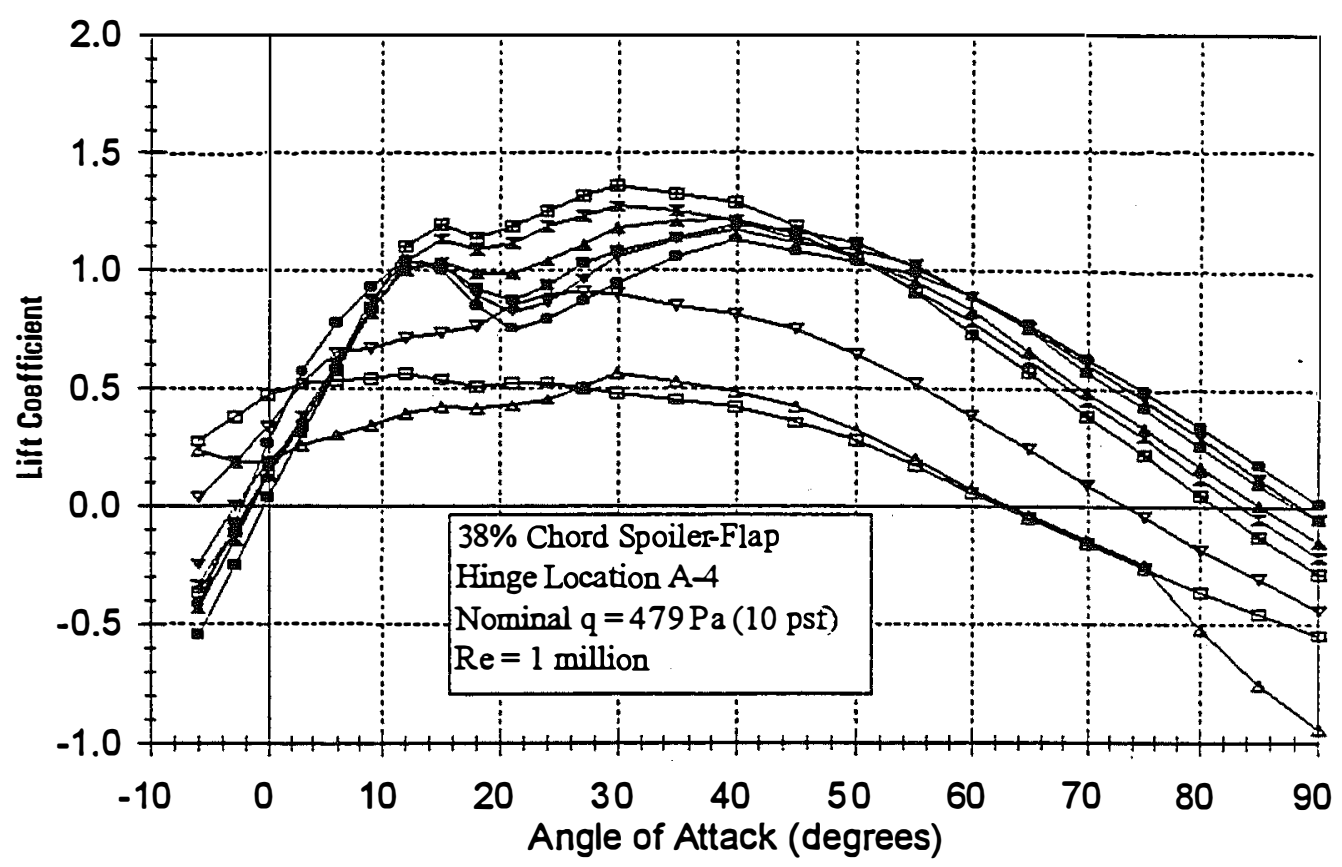

$\rightarrow$ Delta $=0 \rightarrow$ Delta $=5 \rightarrow$ Delta $=10 \rightarrow$ Delta $=20 \rightarrow$ Delta $=30$
$\rightarrow$ Delta $=40 \rightarrow$ Delta $=60 \rightarrow$ Delta $=75 \rightarrow$ Delta $=90$

Figure A-3a. Lift Data, $\delta$-Sweep for $38 \%$ Chord Spoilef-Flap, Hinge A-4

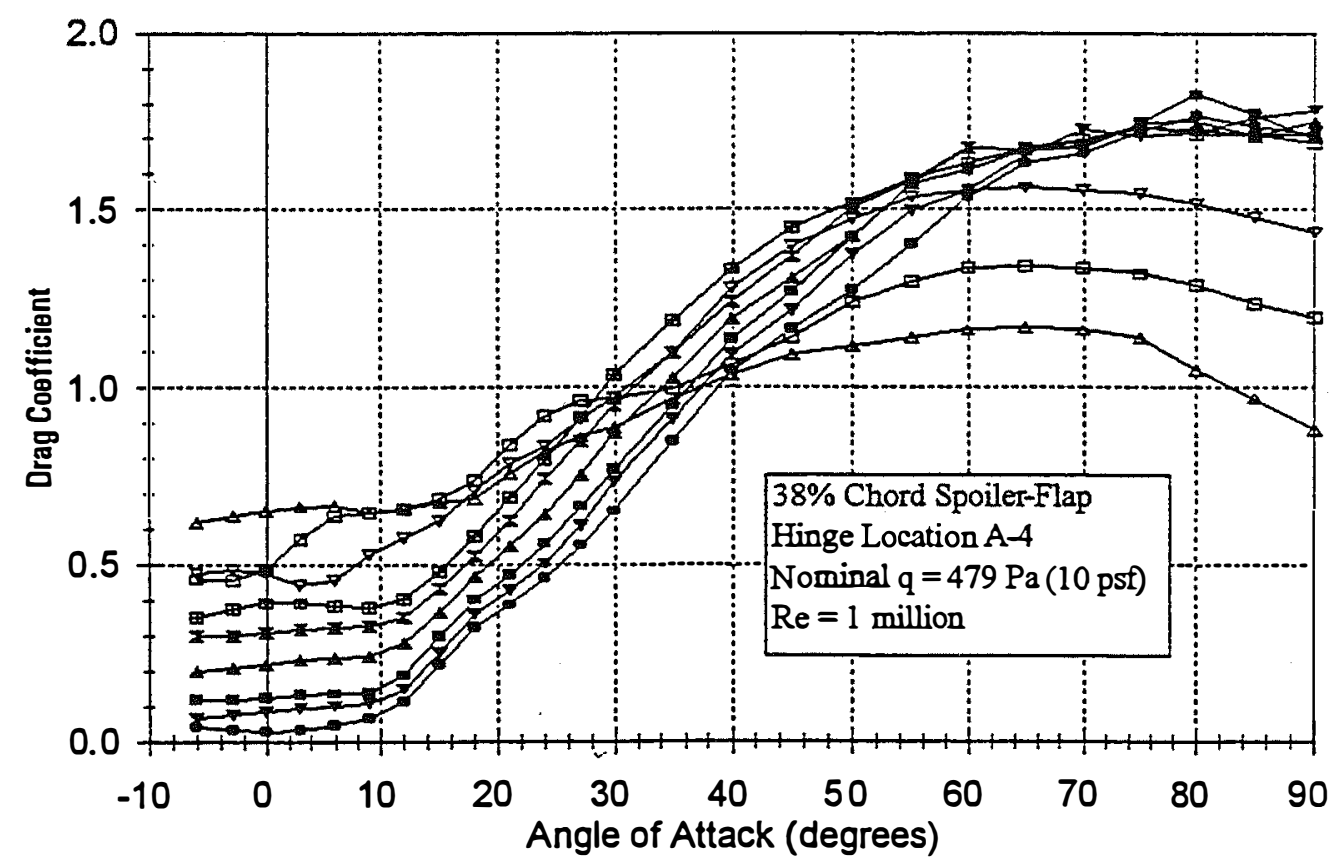

$$
\begin{aligned}
& \rightarrow \text { Delta }=0 \rightarrow \text { Delta }=5 \rightarrow \text { Delta }=10 \rightarrow \text { Delta }=20 \rightarrow \text { Delta }=30 \\
& \rightarrow \text { Delta }=40 \rightarrow \text { Delta }=60 \rightarrow \text { Delta }=75 \rightarrow \text { Delta }=90
\end{aligned}
$$

Figure A-3b. Drag Data, $\delta$-Sweep for $38 \%$ Chord Spoiler-Flap, Hinge A-4 


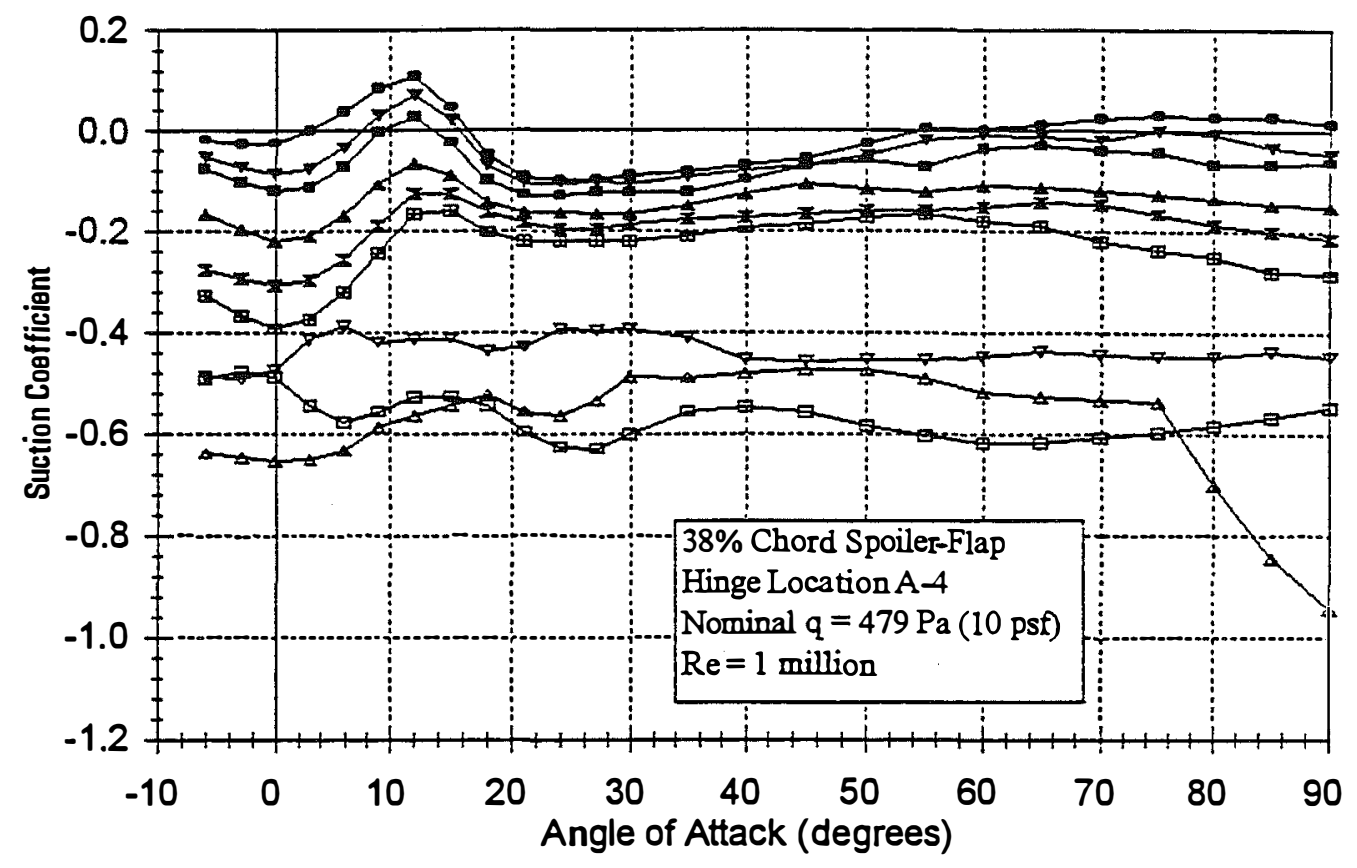

$$
\begin{aligned}
& \rightarrow \text { Delta }=0 \rightarrow \text { Delta }=5 \rightarrow \text { Delta }=10 \rightarrow \text { Delta }=20 \rightarrow \text { Delta }=30 \\
& \rightarrow \text { Delta }=40 \rightarrow \text { Delta }=60 \rightarrow \text { Delta }=75 \rightarrow \text { Delta }=90
\end{aligned}
$$

Figure A-3C. Suction Data, $\delta$-Sweep for $38 \%$ Chord Spoilef-Flap, Hinge A-4

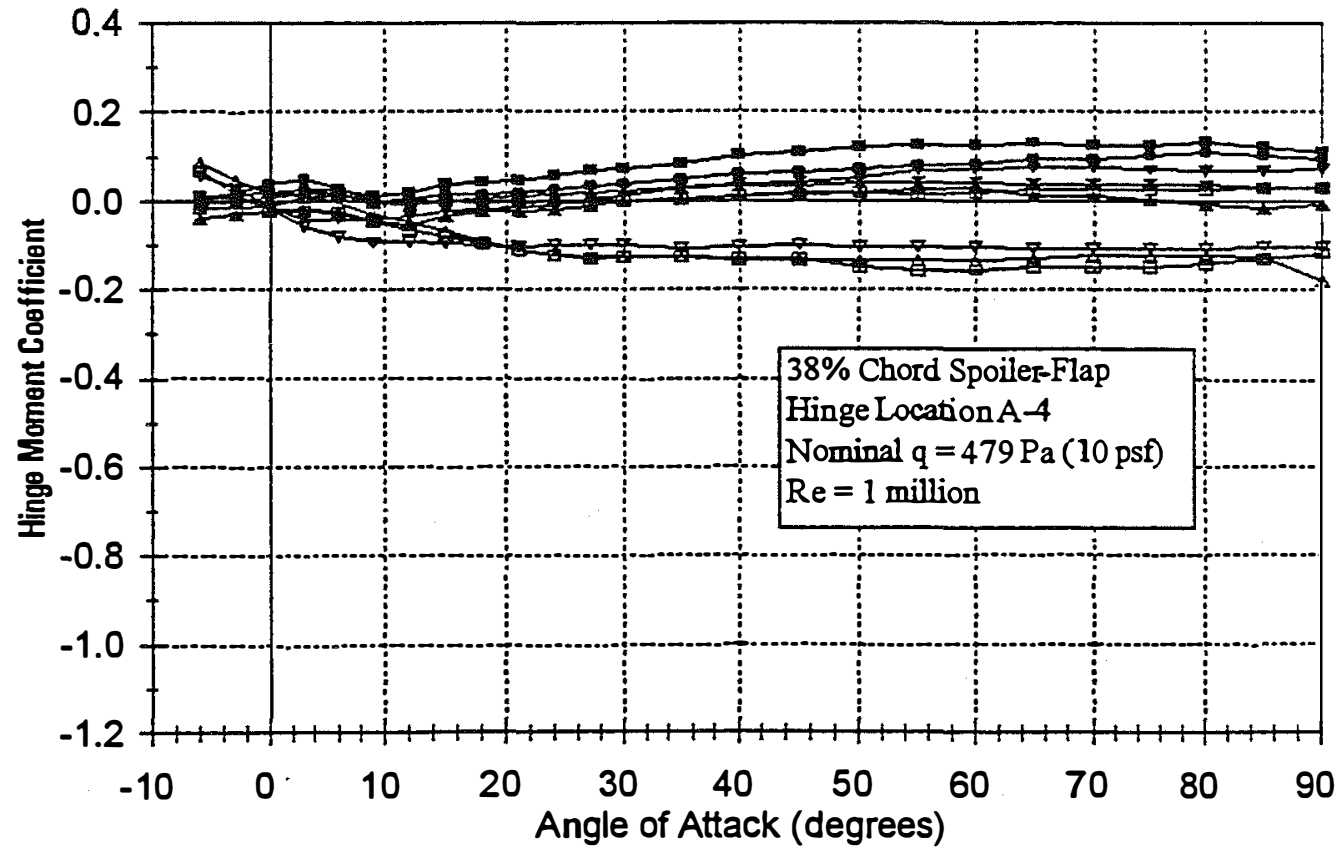

$$
\begin{aligned}
& - \text { Delta }=0 \rightarrow \text { Delta }=5 \rightarrow \text { Delta }=10 \rightarrow \text { Delta }=20 \rightarrow \text { Delta }=30 \\
& \rightarrow \text { Delta }=40 \rightarrow \text { Delta }=60 \rightarrow \text { Delta }=75 \rightarrow \text { Delta }=90
\end{aligned}
$$

Figure A-3d. Hinge Moment Data, $\delta$-Sweep for $38 \%$ Chord Spoiler-Flap, Hinge A-4 


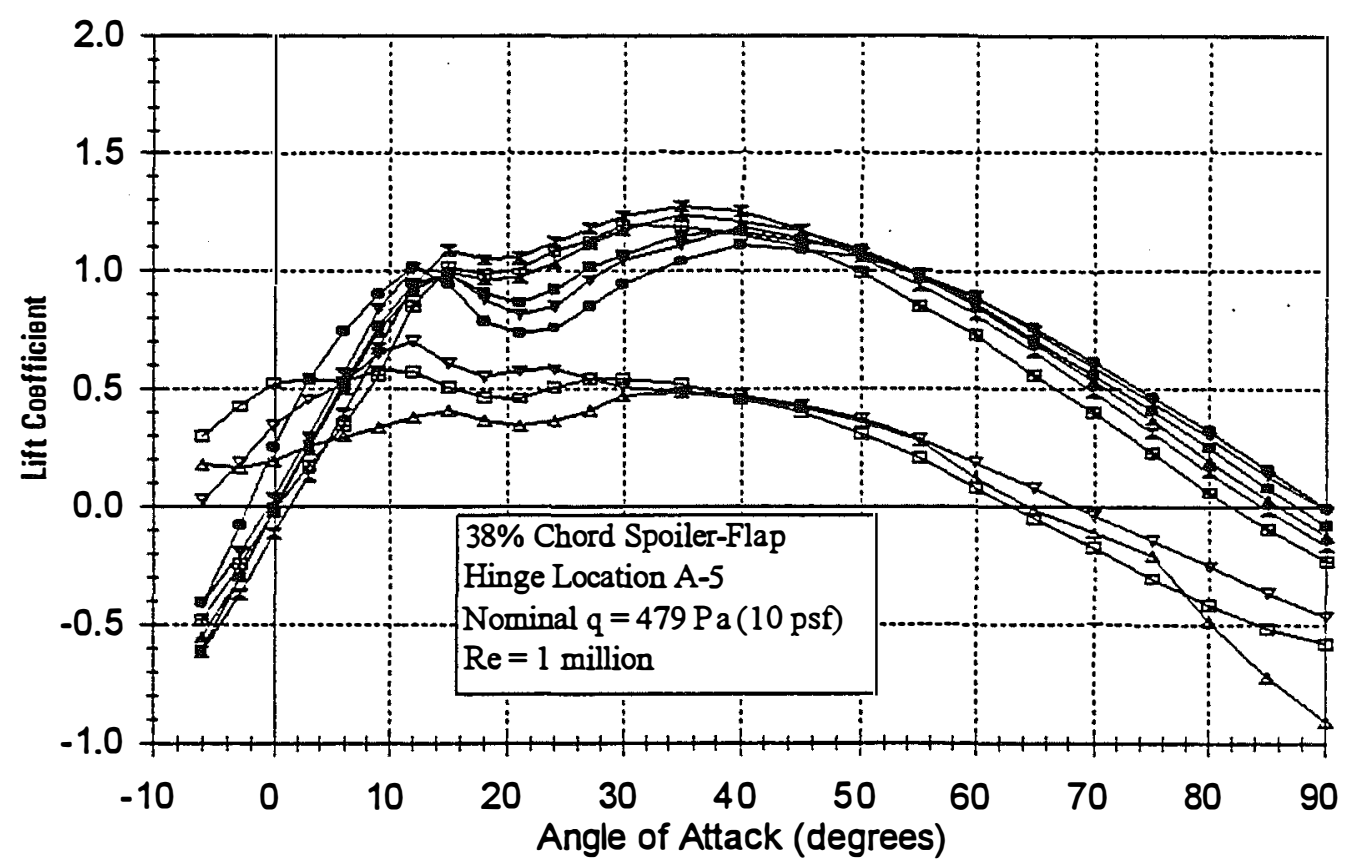

$$
\begin{aligned}
& \rightarrow \text { Delta }=0 \rightarrow \text { Delta }=5 \rightarrow \text { Delta }=10 \rightarrow \text { Delta }=20 \rightarrow \text { Delta }=30 \\
& \rightarrow \text { Delta }=40 \rightarrow \text { Delta }=60 \rightarrow \text { Delta }=75 \rightarrow \text { Delta }=90
\end{aligned}
$$

Figure A-4a. Lift Data, $\delta$-Sweep for $38 \%$ Chord Spoiler-Flap, Hinge A-5

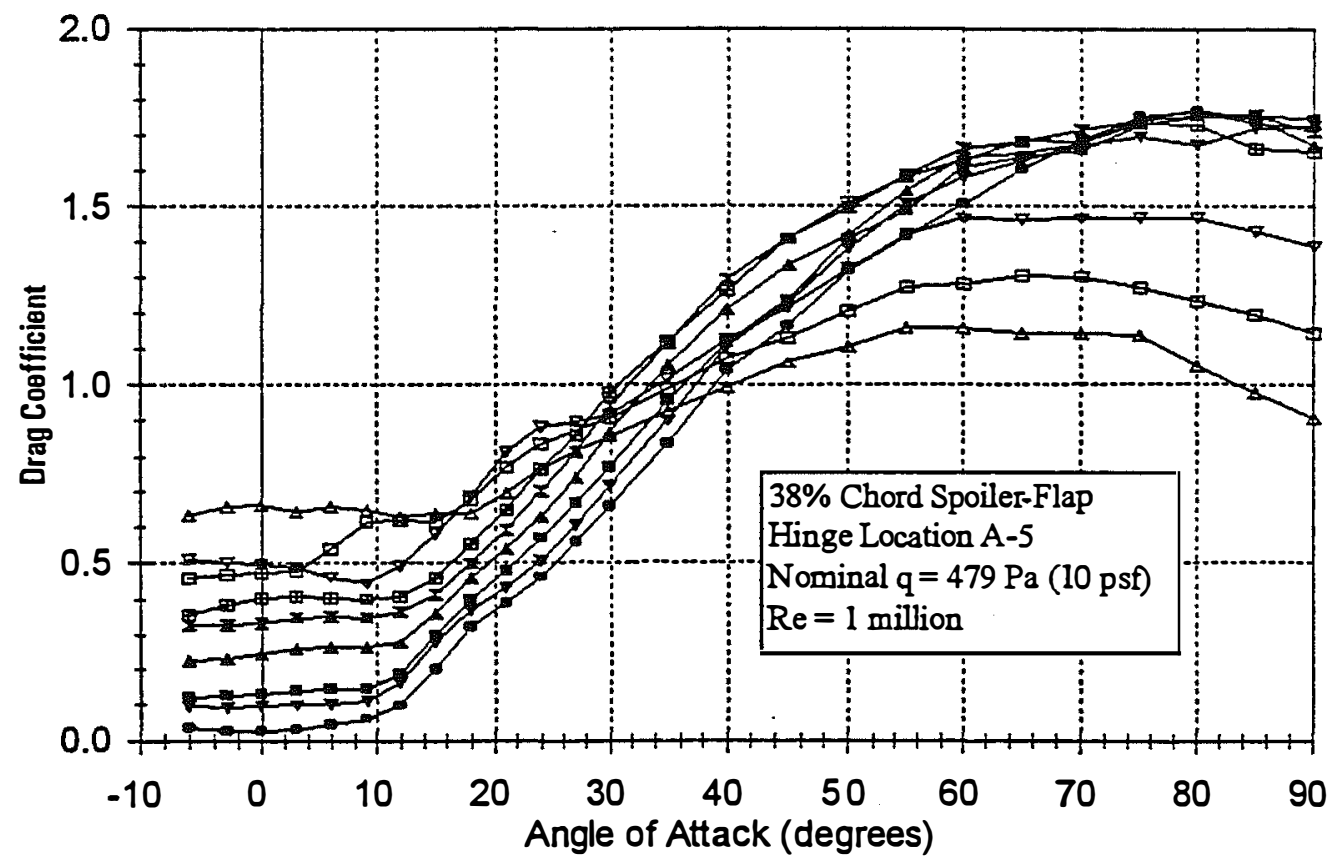

$$
\begin{aligned}
& \rightarrow \text { Delta }=0 \rightarrow \text { Delta }=5 \rightarrow \text { Delta }=10 \rightarrow \text { Delta }=20 \rightarrow \text { Delta }=30 \\
& \rightarrow \text { Delta }=40 \rightarrow \text { Delta }=60 \rightarrow \text { Delta }=75 \rightarrow \text { Delta }=90
\end{aligned}
$$

Figure A-4b. Drag Data, $\delta$-Sweep for $38 \%$ Chord Spoiler-Flap, Hinge A-5 


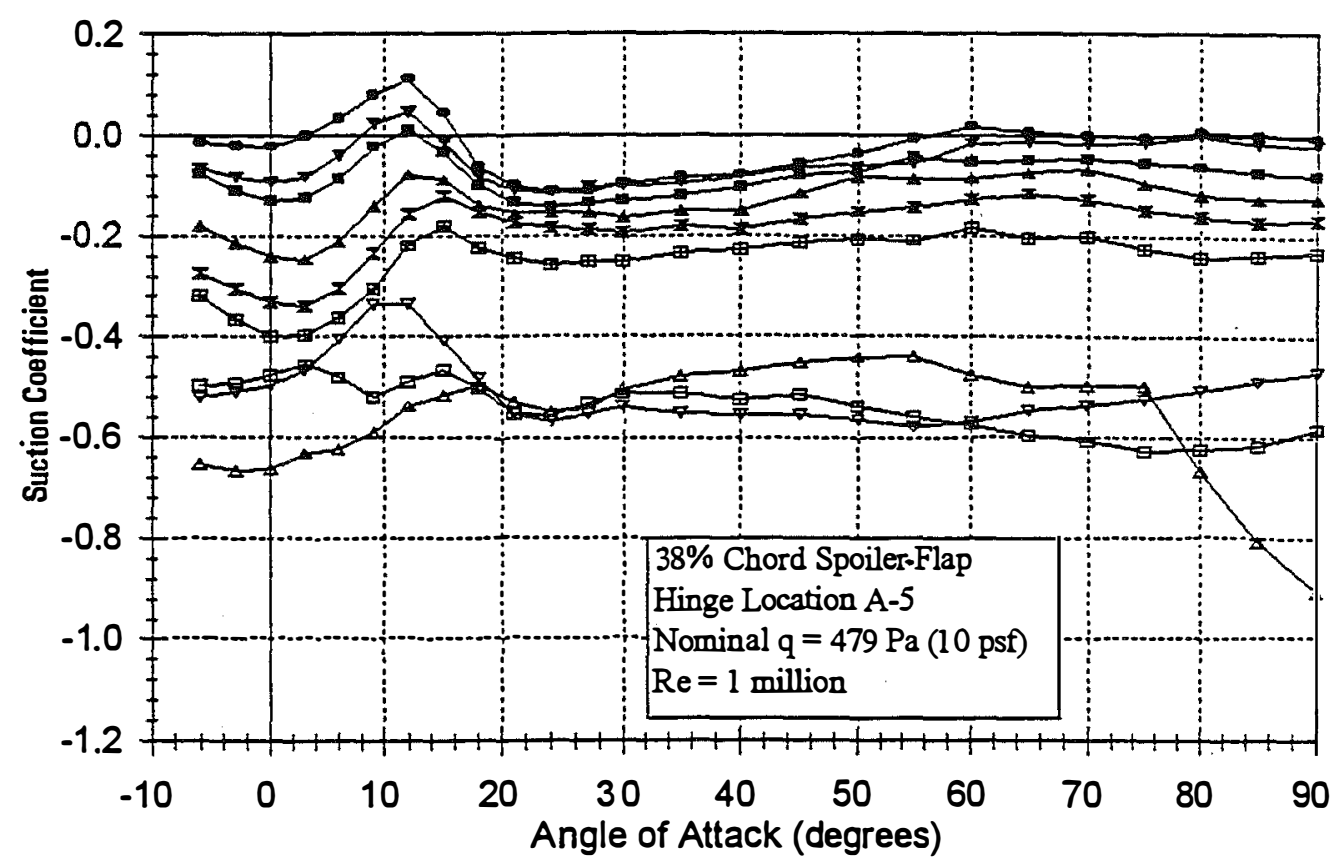

$\rightarrow$ Delta $=0 \rightarrow$ Delta $=5 \rightarrow$ Delta $=10 \rightarrow$ Delta $=20 \rightarrow$ Delta $=30$

$\rightarrow$ Delta $=40 \rightarrow$ Delta $=60 \rightarrow$ Delta $=75 \rightarrow$ Delta $=90$

Figure A-4c. Suction Data, $\delta$-Sweep for $38 \%$ Chord Spoiler-Flap, Hinge A-5

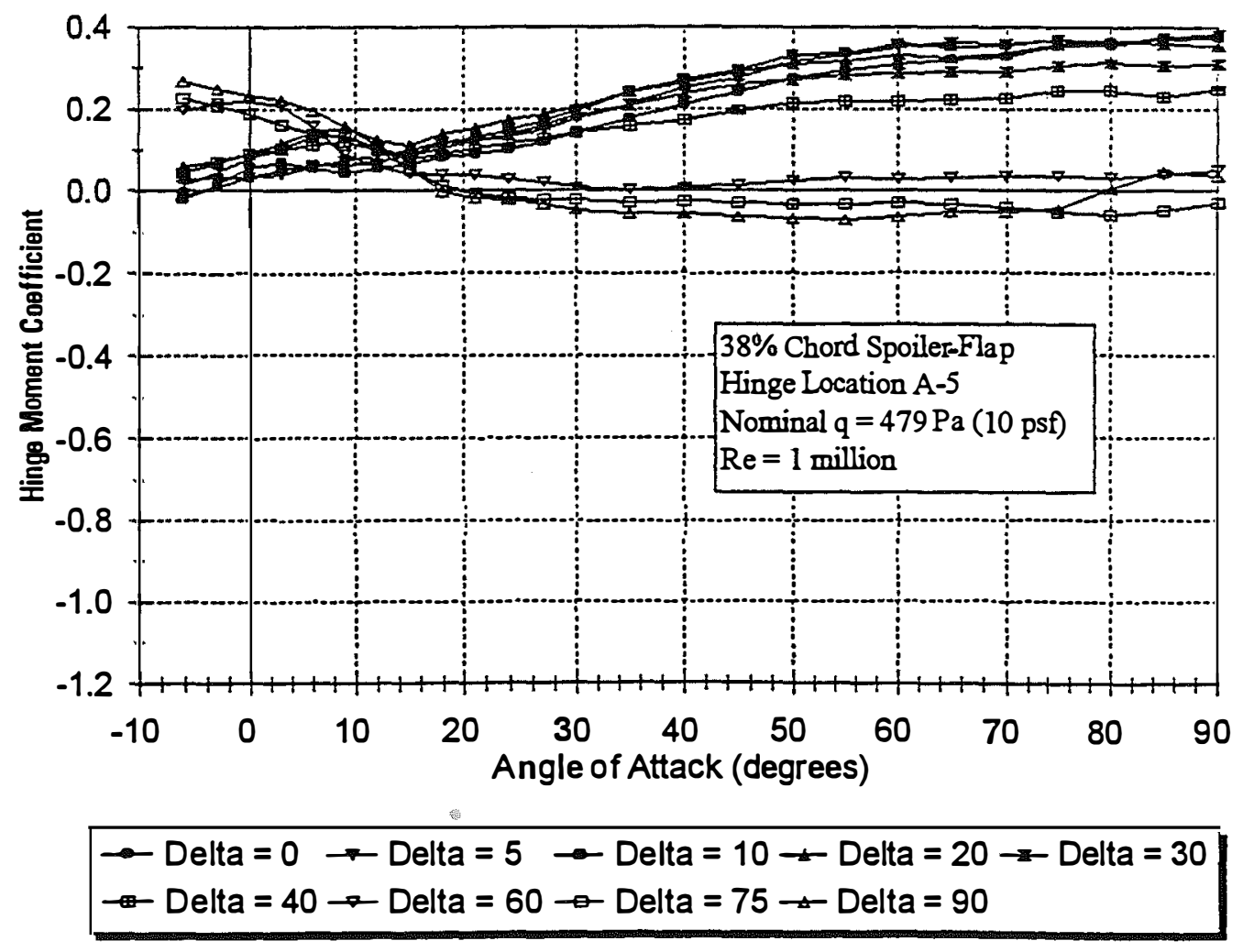

Figure A-4d. Hinge Moment Data, $\delta-S w e e p$ for $38 \%$ Chord Spoiler-Flap, Hinge A-5 


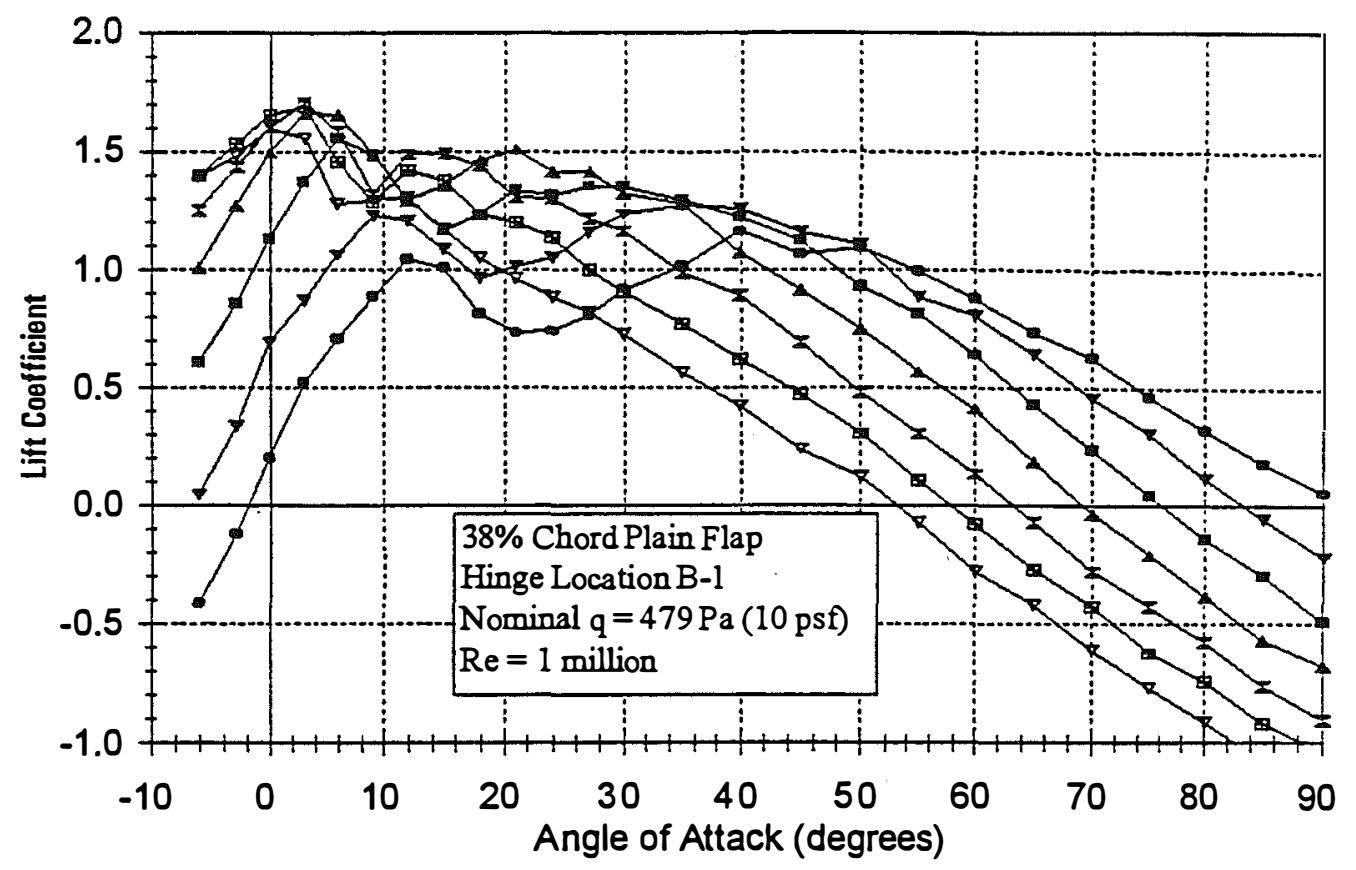

$$
\begin{aligned}
& \rightarrow \text { Delta }=0 \rightarrow \text { Delta }=15 \rightarrow \text { Delta }=30 \rightarrow \text { Delta }=45 \\
& \rightarrow \text { Delta }=60 \rightarrow \text { Delta }=75 \rightarrow \text { Delta }=90
\end{aligned}
$$

Figure A-5a. Lift Data, $\delta$ Sweep for $38 \%$ Chord Plain Flap, Hinge B-1

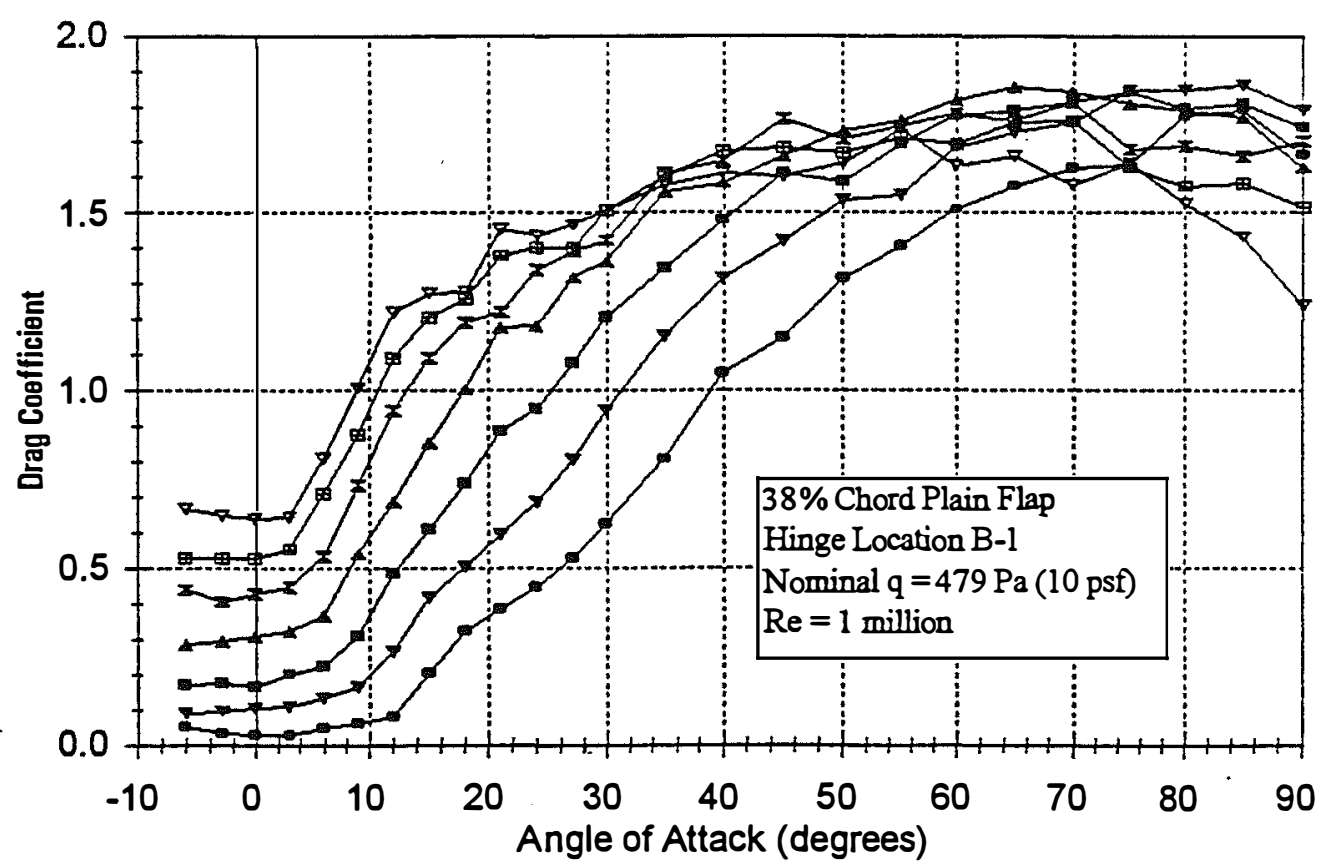

$$
\begin{aligned}
& \rightarrow \text { Delta }=0 \rightarrow \text { Delta }=15 \rightarrow \text { Delta }=30 \rightarrow \text { Delta }=45 \\
& \rightarrow \text { Delta }=60 \rightarrow \text { Delta }=75 \rightarrow \text { Delta }=90
\end{aligned}
$$

Figure A-5b. Drag Data, $\delta-5 w e e p$ for $38 \%$ Chord Plain Flap, Hinge B-1 


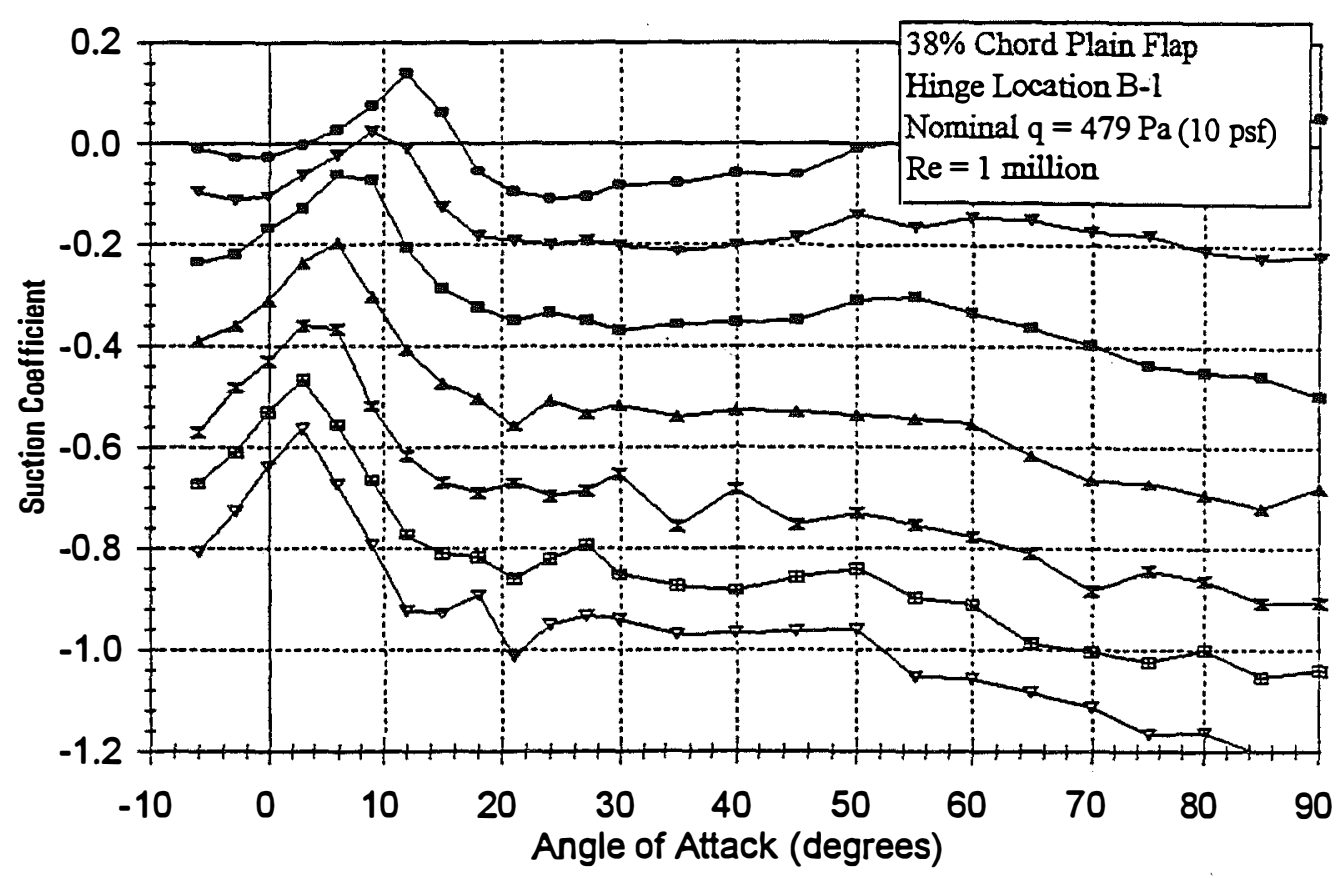

$\rightarrow$ Delta $=0 \rightarrow$ Delta $=15 \rightarrow$ Delta $=30 \rightarrow$ Delta $=45$
$\rightarrow$ Delta $=60 \rightarrow$ Delta $=75 \rightarrow$ Delta $=90$

Figure A-5c. Suction Data, $\delta$-Sweep for $38 \%$ Chord Plain Flap, Hinge B-1

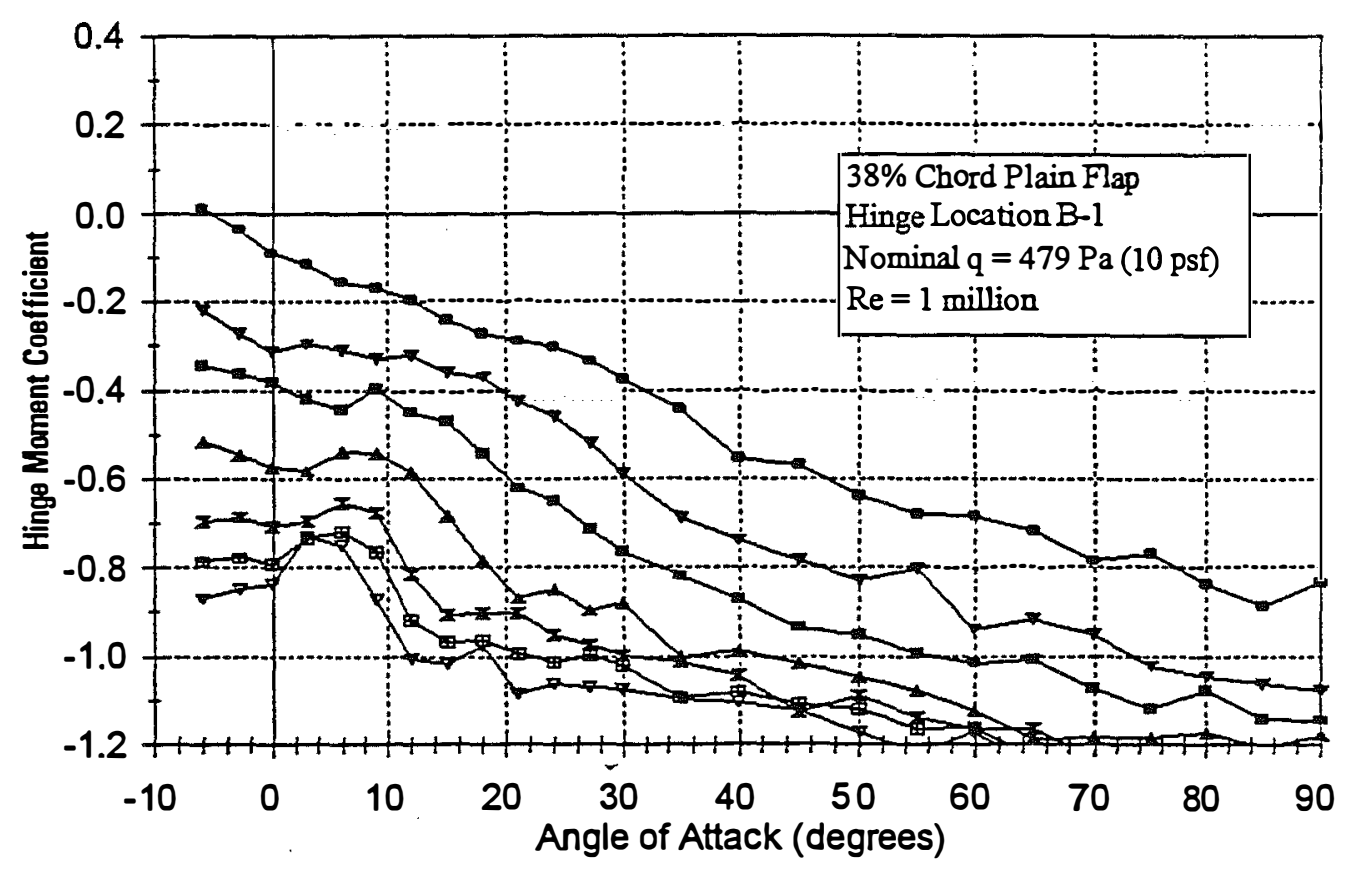

$$
\begin{aligned}
& \rightarrow \text { Delta }=0 \rightarrow \text { Delta }=15 \rightarrow \text { Delta }=30 \quad \text { Delta }=45 \\
& \rightarrow \text { Delta }=60 \rightarrow \text { Delta }=75 \rightarrow \text { Delta }=90
\end{aligned}
$$

Figure A-5d. Hinge Moment Data, $\delta-S w e e p$ for $38 \%$ Chord Plain Flap, Hinge B-1 


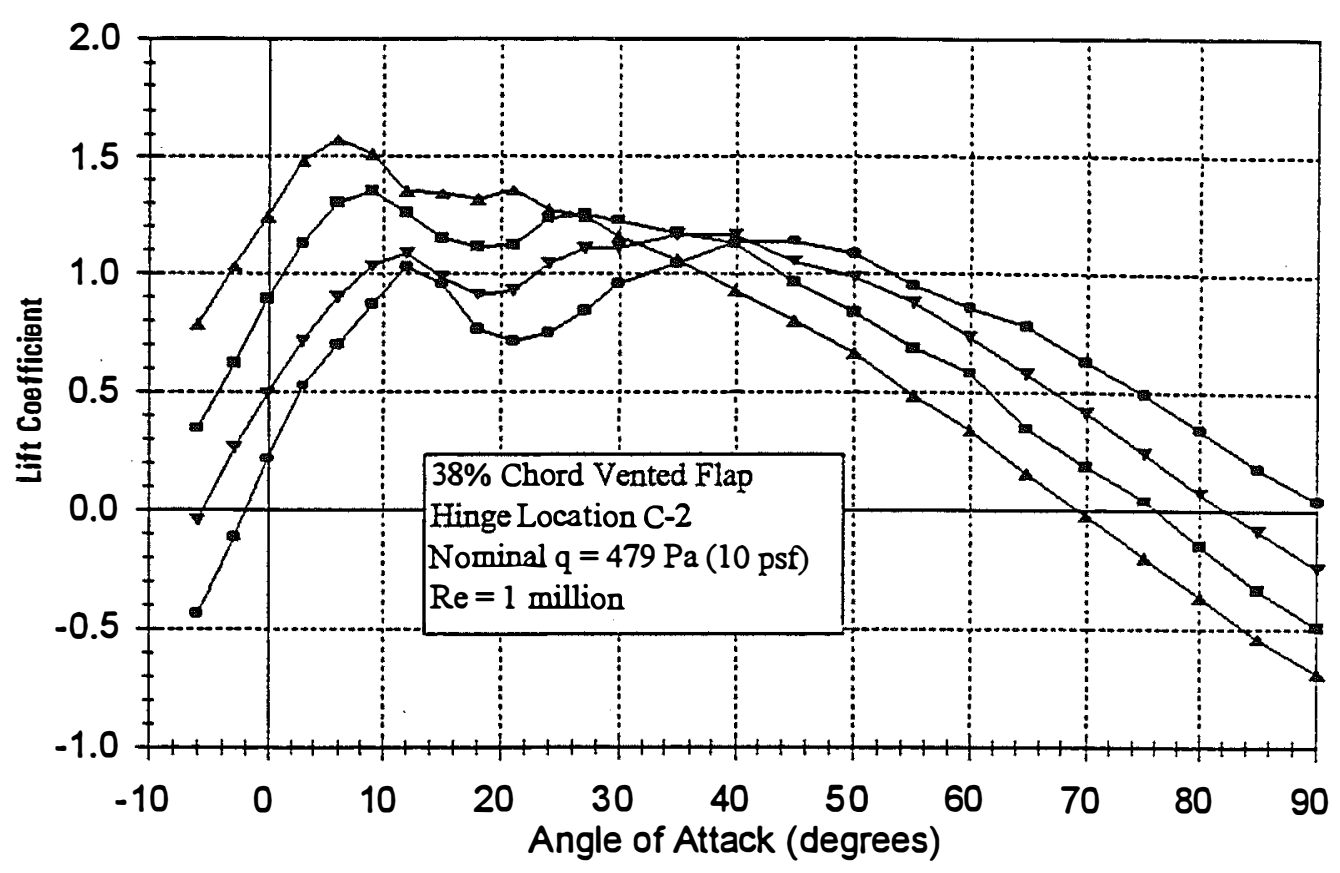

$\rightarrow$ Delta $=0 \rightarrow$ Delta $=15 \rightarrow$ Delta $=30 \multimap$ Delta $=45$

Figure A-6a. Lift Data, $\delta$ Sweep for $38 \%$ Chord Vented Flap, Hinge C-2

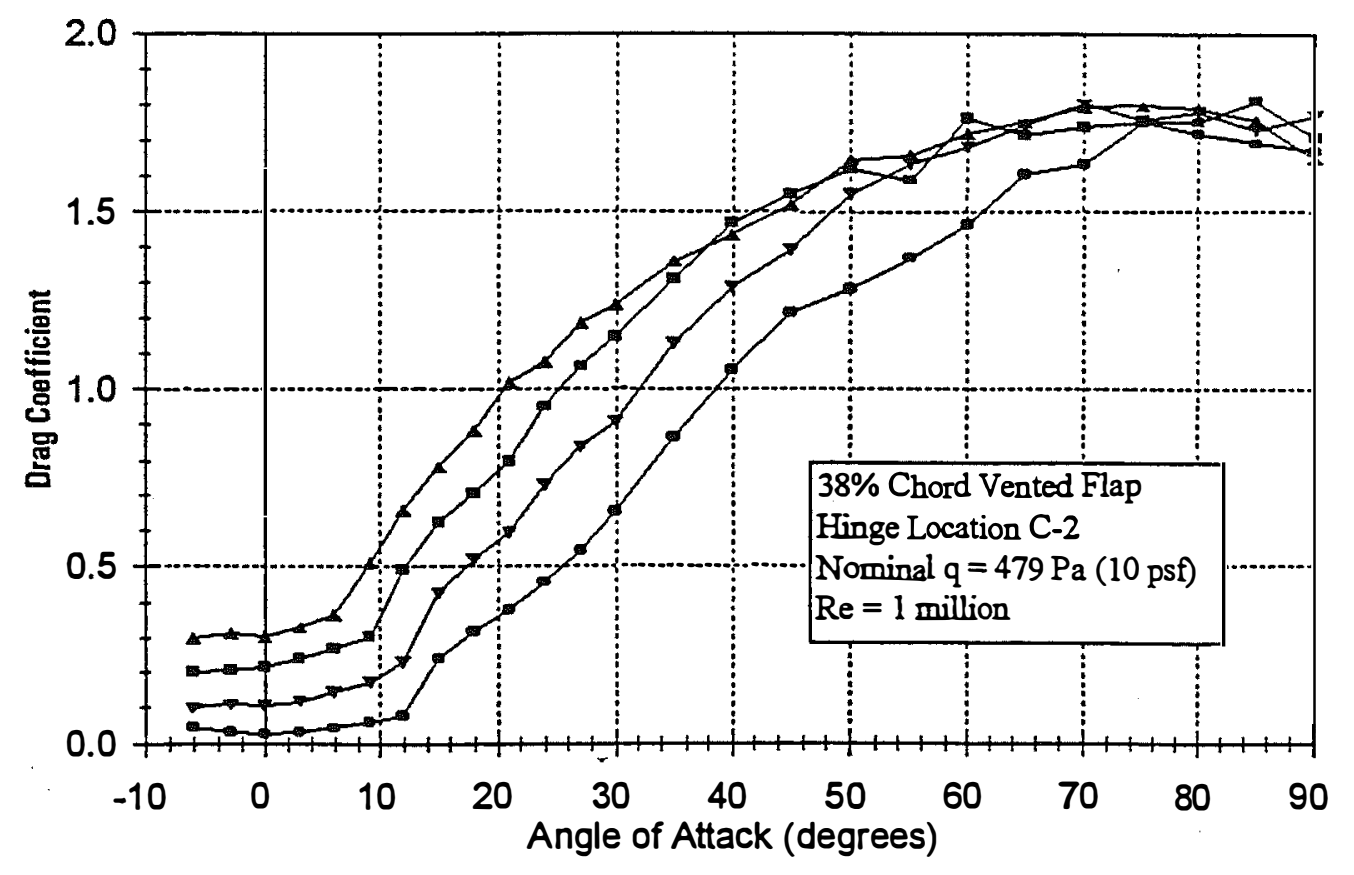

$\rightarrow$ Delta $=0 \rightarrow$ Delta $=15 \rightarrow$ Delta $=30 \rightarrow$ Delta $=45$

Figure A-6b. Drag Data, $\delta$ Sweep for $38 \%$ Chord Vented Flap, Hinge C-2 


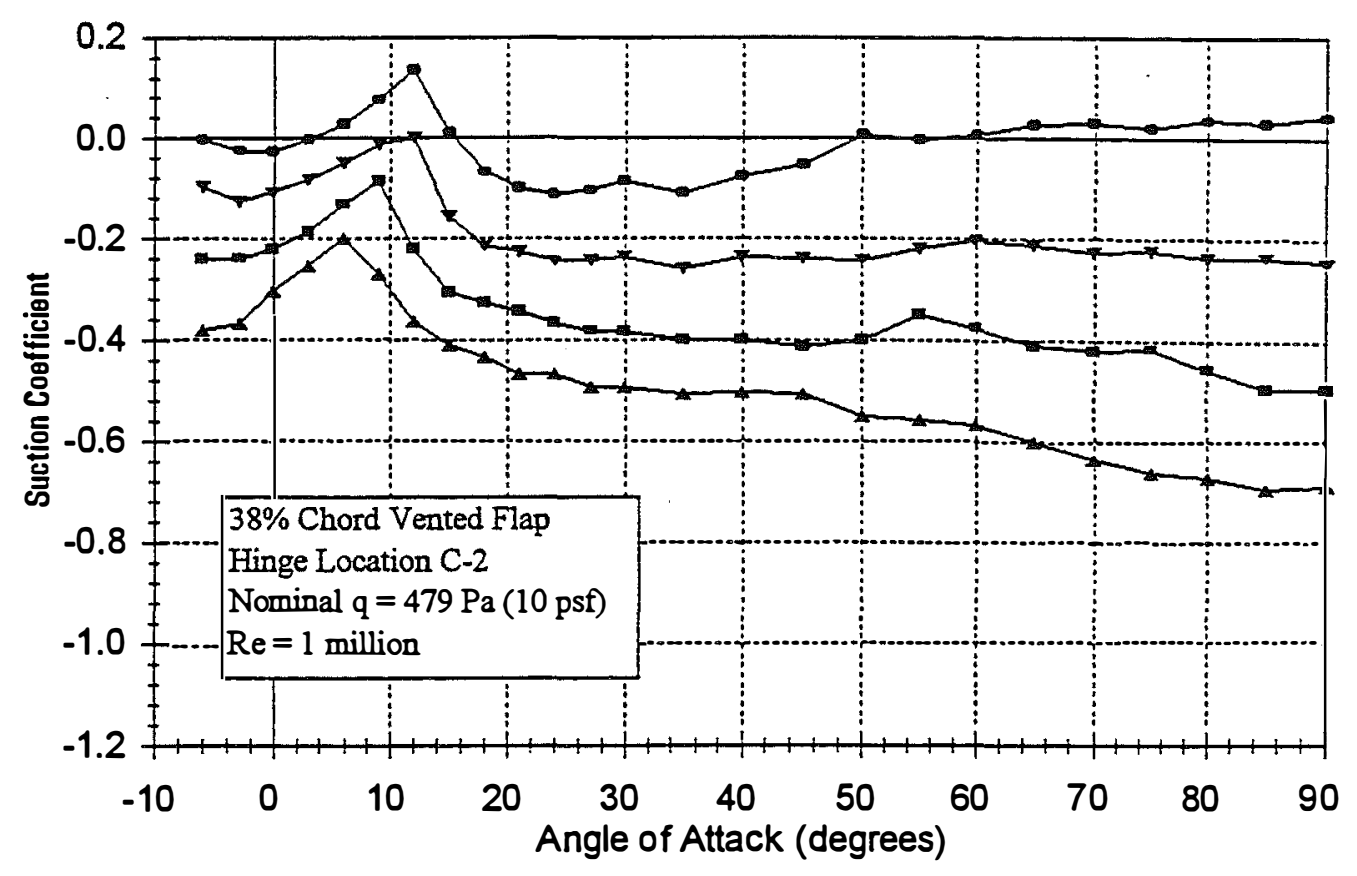

$\rightarrow$ Delta $=0 \rightarrow$ Delta $=15 \rightarrow$ Delta $=30 \rightarrow$ Delta $=45$

Figure A-6c. Suction Data, $\delta$-Sweep for $38 \%$ Chord Vented Flap, Hinge C-2

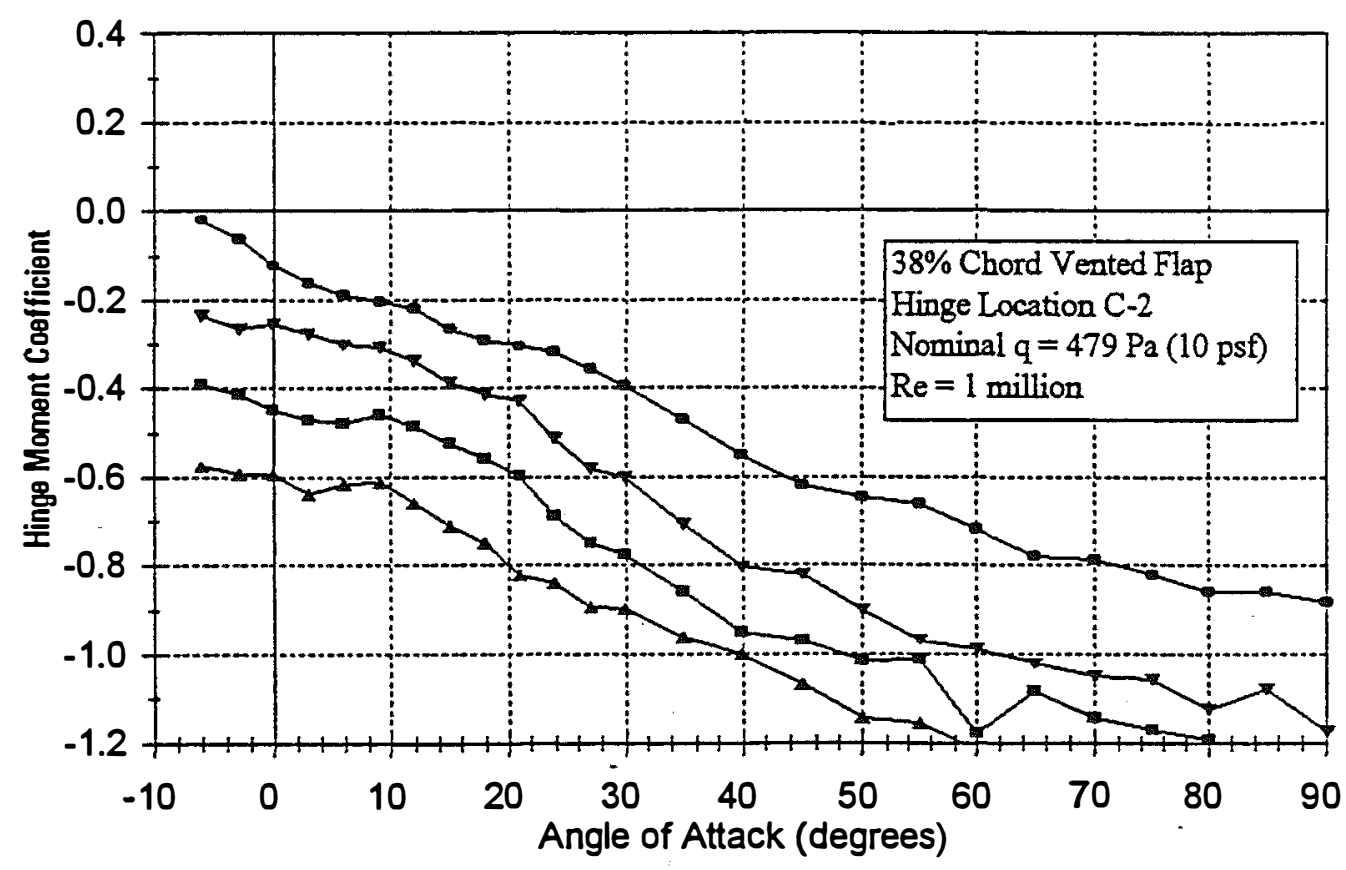

$\rightarrow$ Delta $=0 \rightarrow$ Delta $=15 \rightarrow$ Delta $=30 \rightarrow$ Delta $=45$

Figure A-6d. Hinge Moment Data, $\delta$-Sweep for $38 \%$ Chord Vented Flap, Hinge C-2 


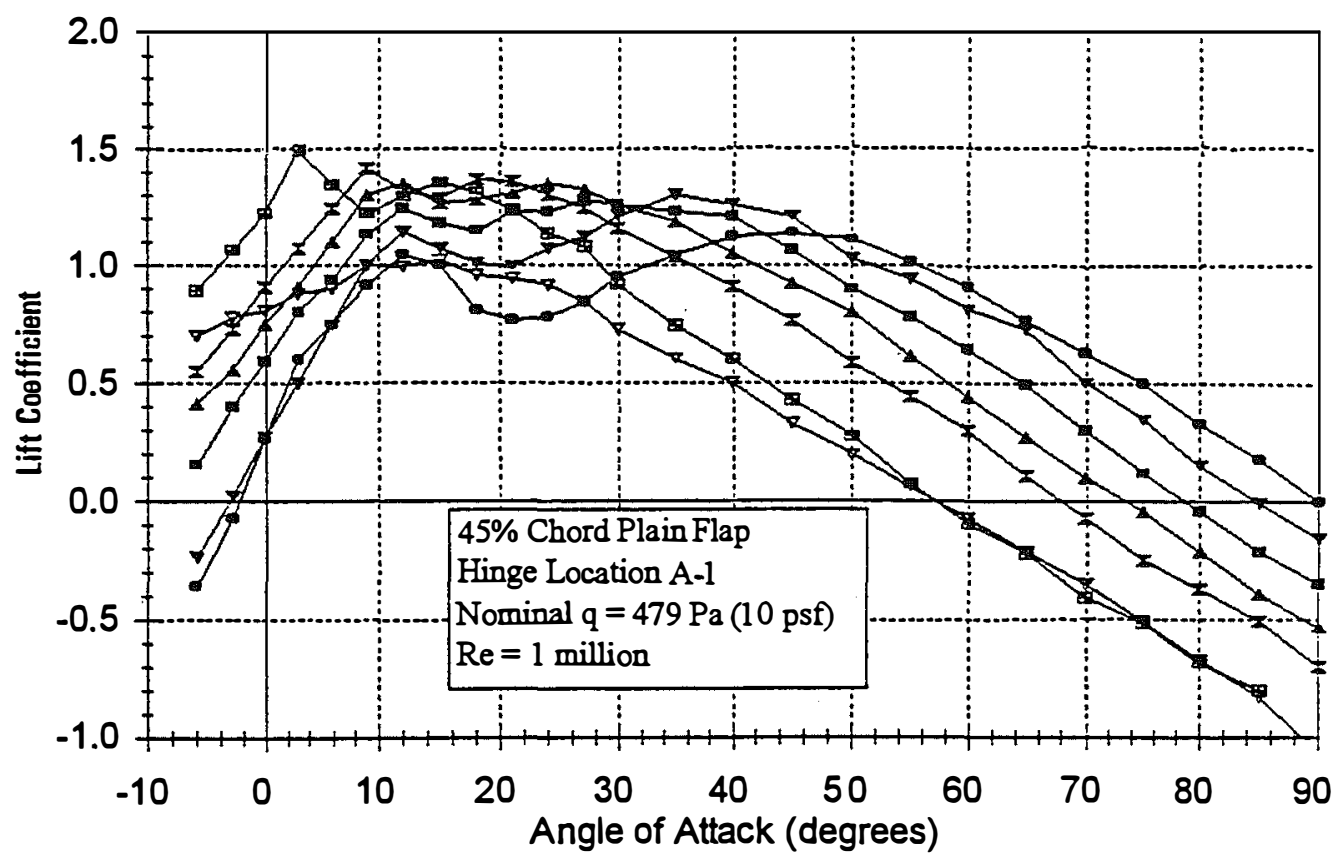

$$
\begin{aligned}
& \rightarrow \text { Delta }=0 \rightarrow \text { Delta }=15 \rightarrow \text { Delta }=30 \rightarrow \text { Delta }=45 \\
& \rightarrow \text { Delta }=60 \rightarrow \text { Delta }=75 \rightarrow \text { Delta }=90
\end{aligned}
$$

Figure A-7a. Lift Data, $\delta$ Sweep for $45 \%$ Chord Plain Flap, Hinge A-1

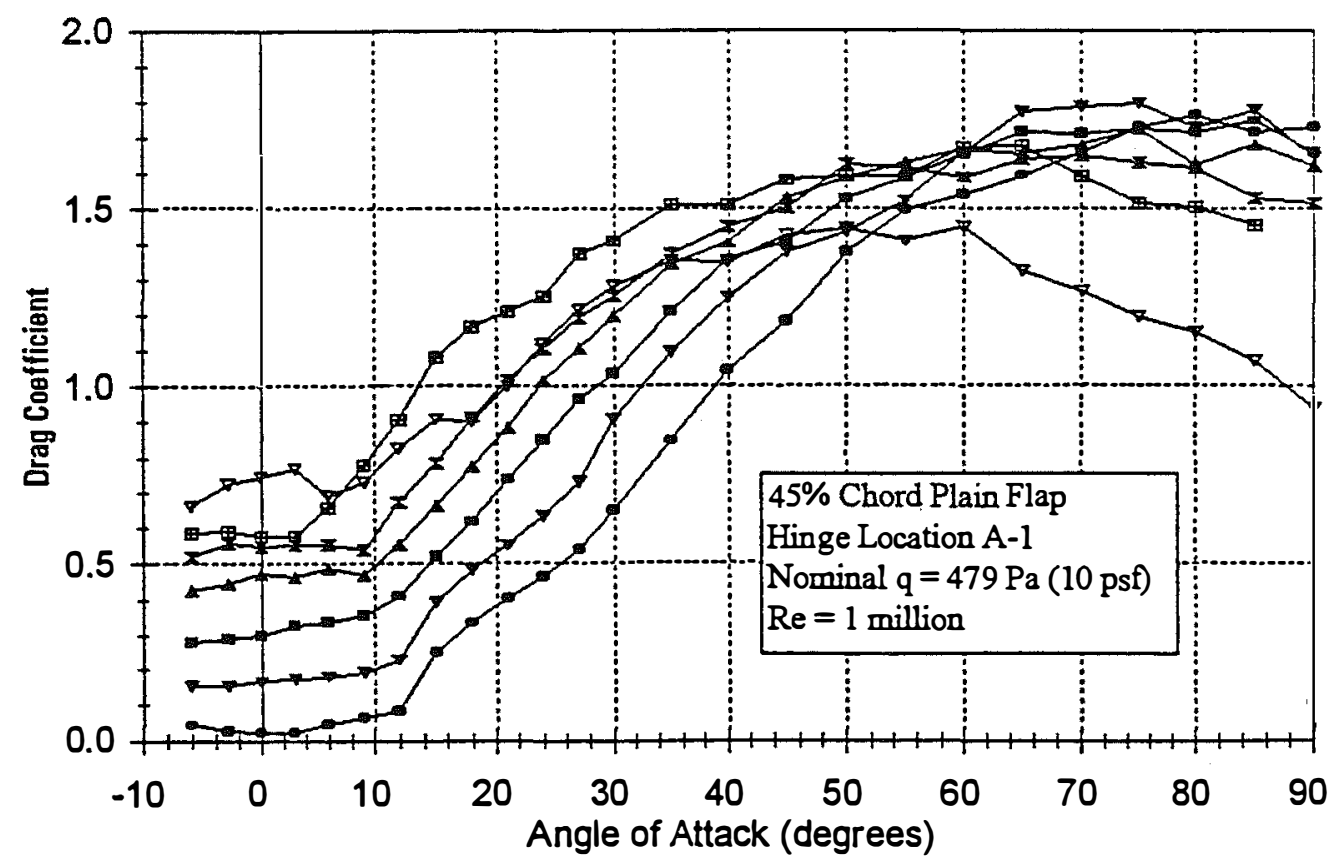

$$
\begin{aligned}
& \rightarrow \text { Delta }=0 \rightarrow \text { Delta }=15 \rightarrow \text { Delta }=30 \rightarrow \text { Delta }=45 \\
& \rightarrow \text { Delta }=60 \rightarrow \text { Delta }=75 \rightarrow \text { Delta }=90
\end{aligned}
$$

Figure A-7b. Drag Data, $\delta$ Sweep for $45 \%$ Chord Plain Flap, Hinge A-1 


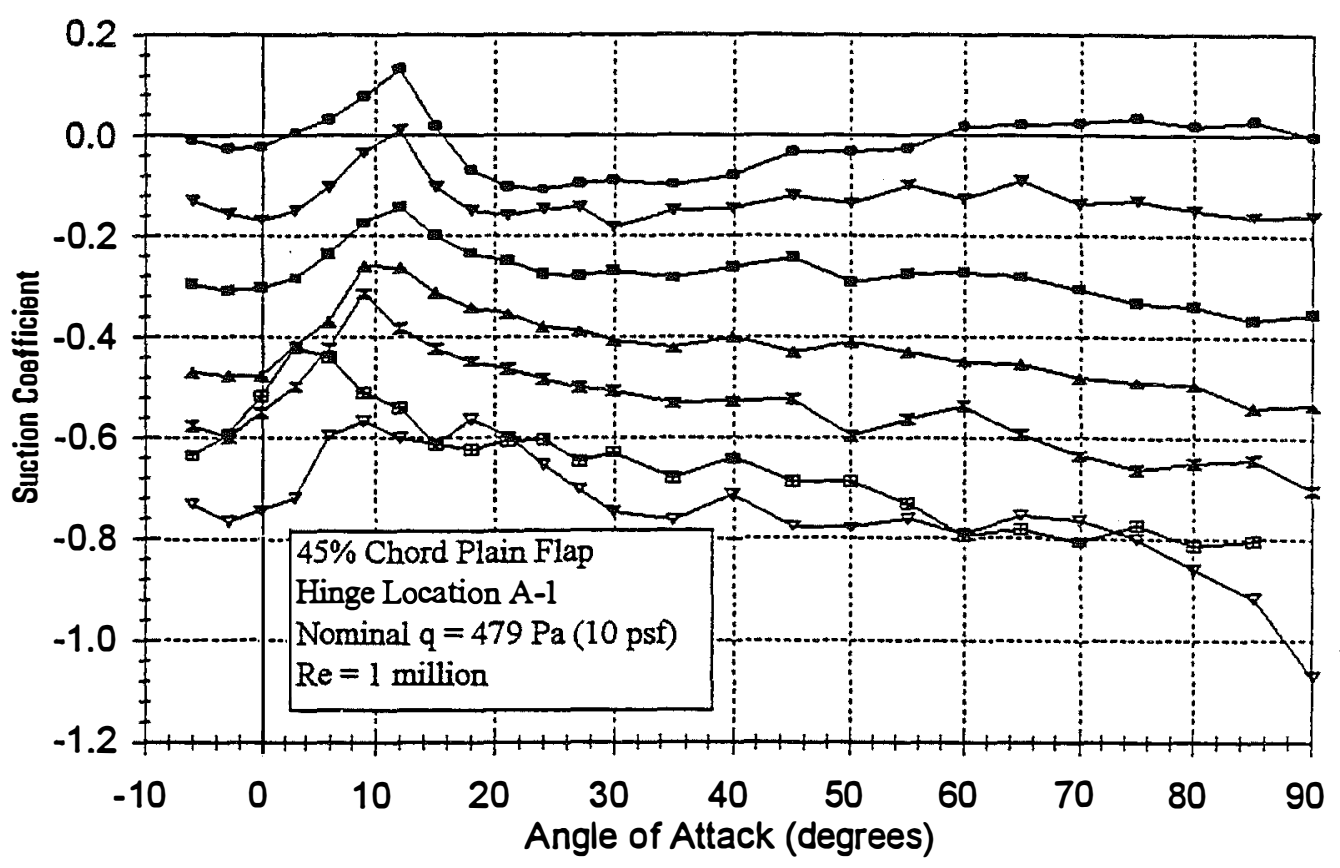

$$
\begin{aligned}
& \rightarrow \text { Delta }=0 \rightarrow \text { Delta }=15 \rightarrow \text { Delta }=30 \rightarrow \text { Delta }=45 \\
& \rightarrow \text { Delta }=60 \rightarrow \text { Delta }=75 \rightarrow \text { Delta }=90
\end{aligned}
$$

Figure A-7c. Suction Data, $\delta$-Sweep for $45 \%$ Chord Plain Flap, Hinge A-1

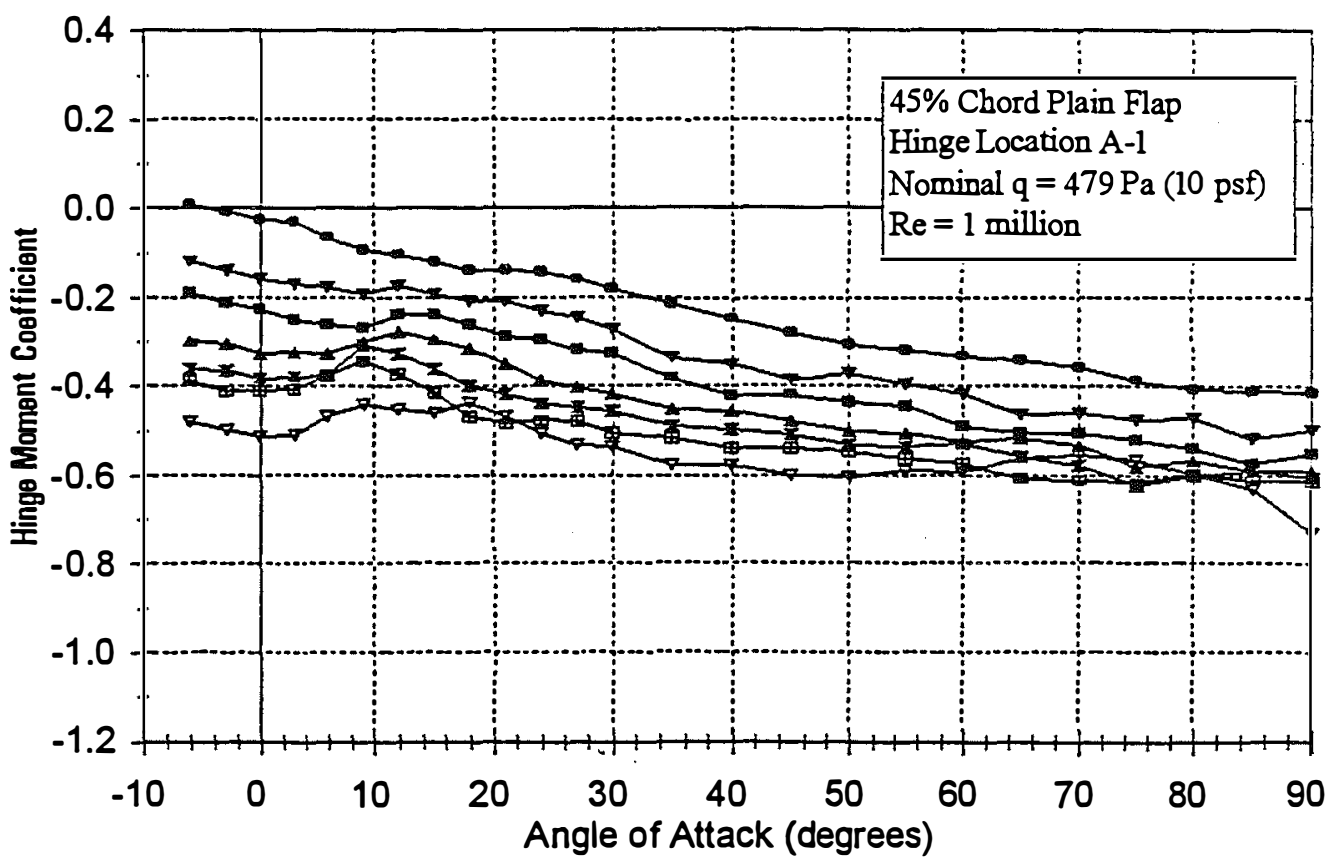

$$
\begin{aligned}
& \rightarrow \text { Delta }=0 \rightarrow \text { Delta }=15 \rightarrow \text { Delta }=30 \rightarrow \text { Delta }=45 \\
& \rightarrow \text { Delta }=60 \rightarrow \text { Delta }=75 \rightarrow \text { Delta }=90
\end{aligned}
$$

Figure A-7d. Hinge Moment Data, $\delta$-Sweep for $\mathbf{4 5} \%$ Chord Plain Flap, Hinge A-1 


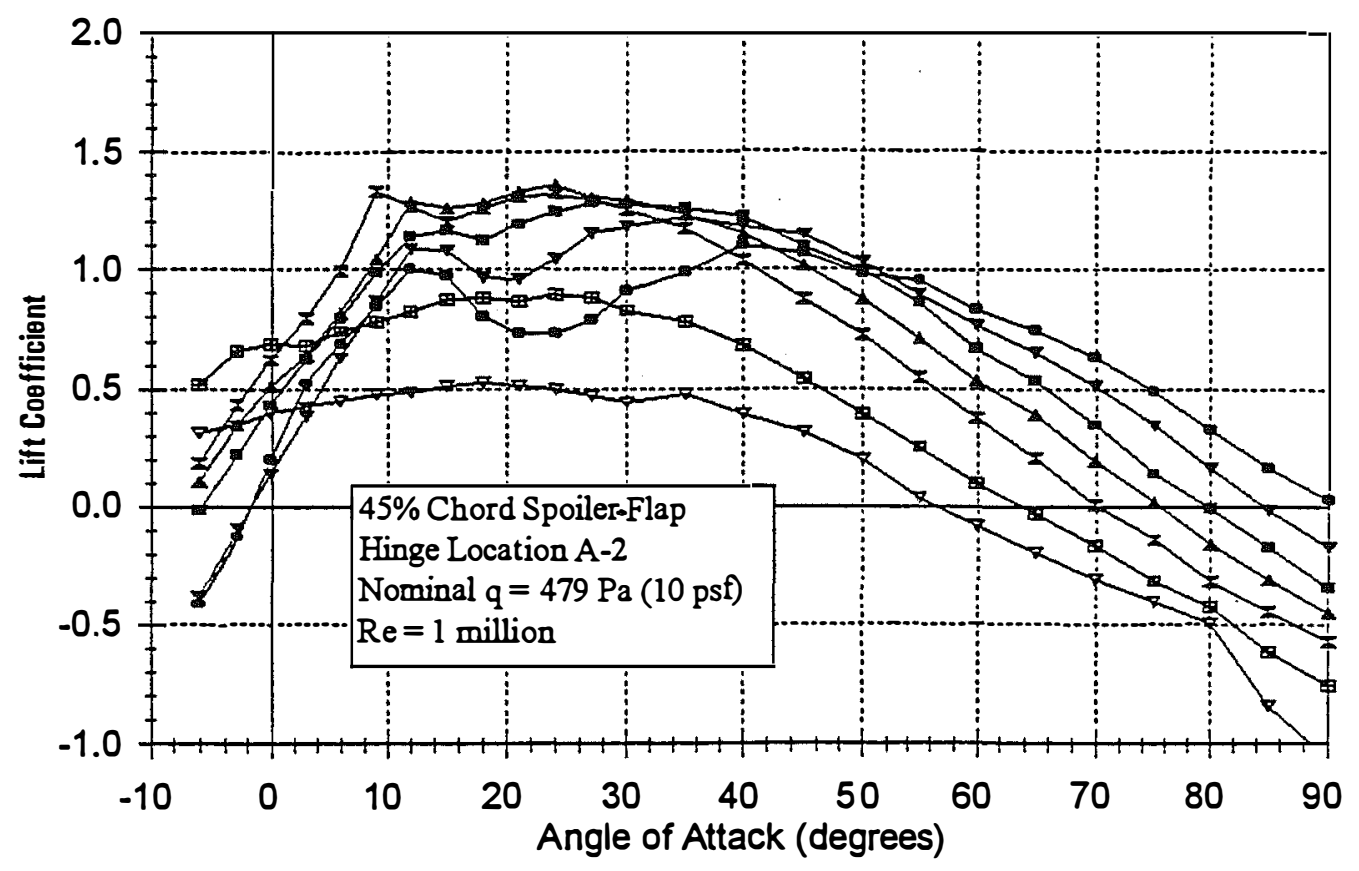

$$
\begin{aligned}
& \rightarrow \text { Delta }=0 \rightarrow \text { Delta }=15 \rightarrow \text { Delta }=30 \multimap \text { Delta }=45 \\
& \rightarrow \text { Delta }=60 \rightarrow \text { Delta }=75 \rightarrow \text { Delta }=90
\end{aligned}
$$

Figure A-8a. Lift Data, $\delta$-Sweep for $45 \%$ Chord Spoiler.Flap, Hinge A-2

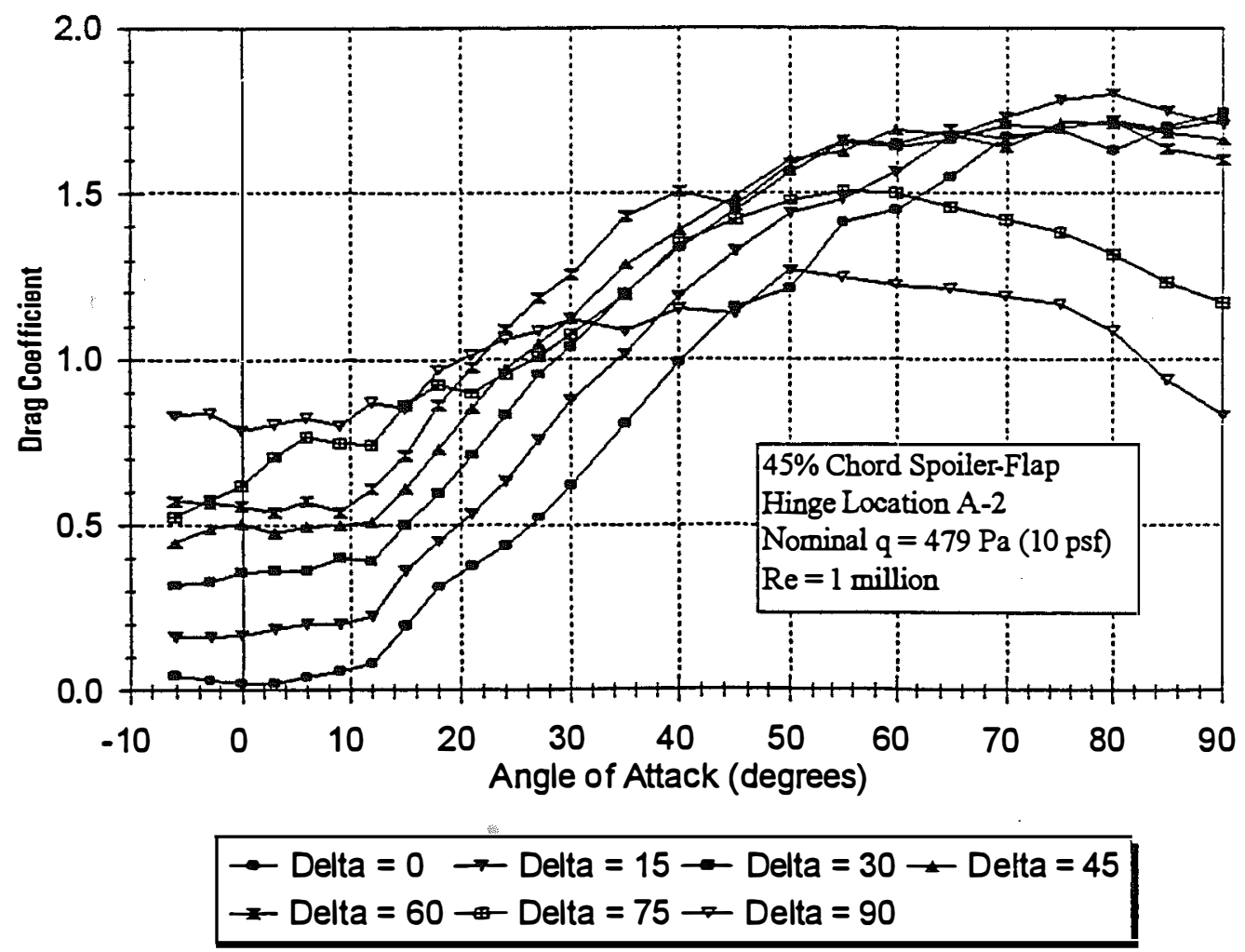

Figure A-8b. Drag Data, $\delta$-Sweep for $45 \%$ Chord Spoiler-Flap, Hinge A-2 


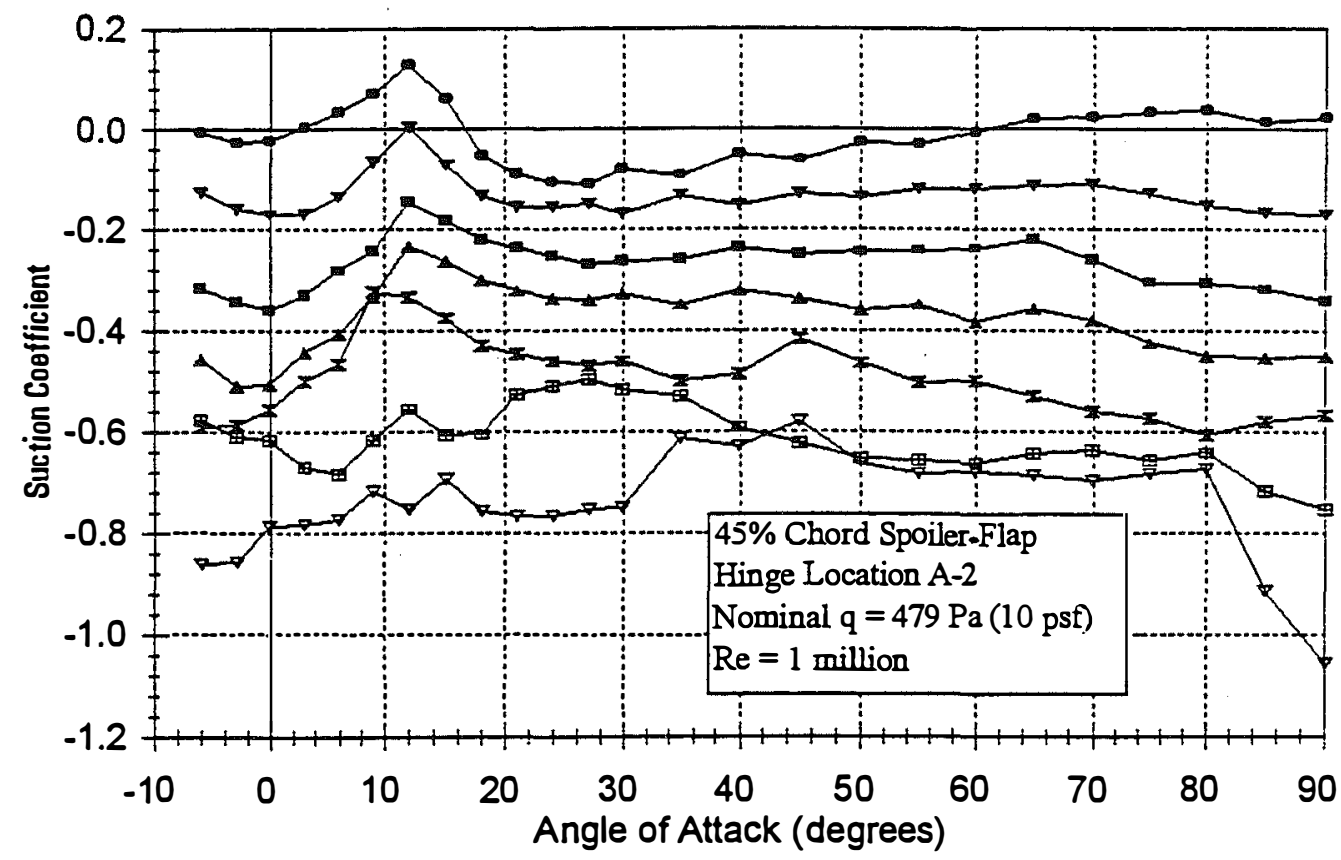

$$
\begin{aligned}
& \rightarrow \text { Delta }=0 \rightarrow \text { Delta }=15 \rightarrow \text { Delta }=30 \rightarrow \text { Delta }=45 \\
& \rightarrow \text { Delta }=60 \rightarrow \text { Delta }=75 \rightarrow \text { Delta }=90
\end{aligned}
$$

Figure A-8c. Suction Data, $\delta$-Sweep for $45 \%$ Chord Spoiler-Flap, Hinge A-2

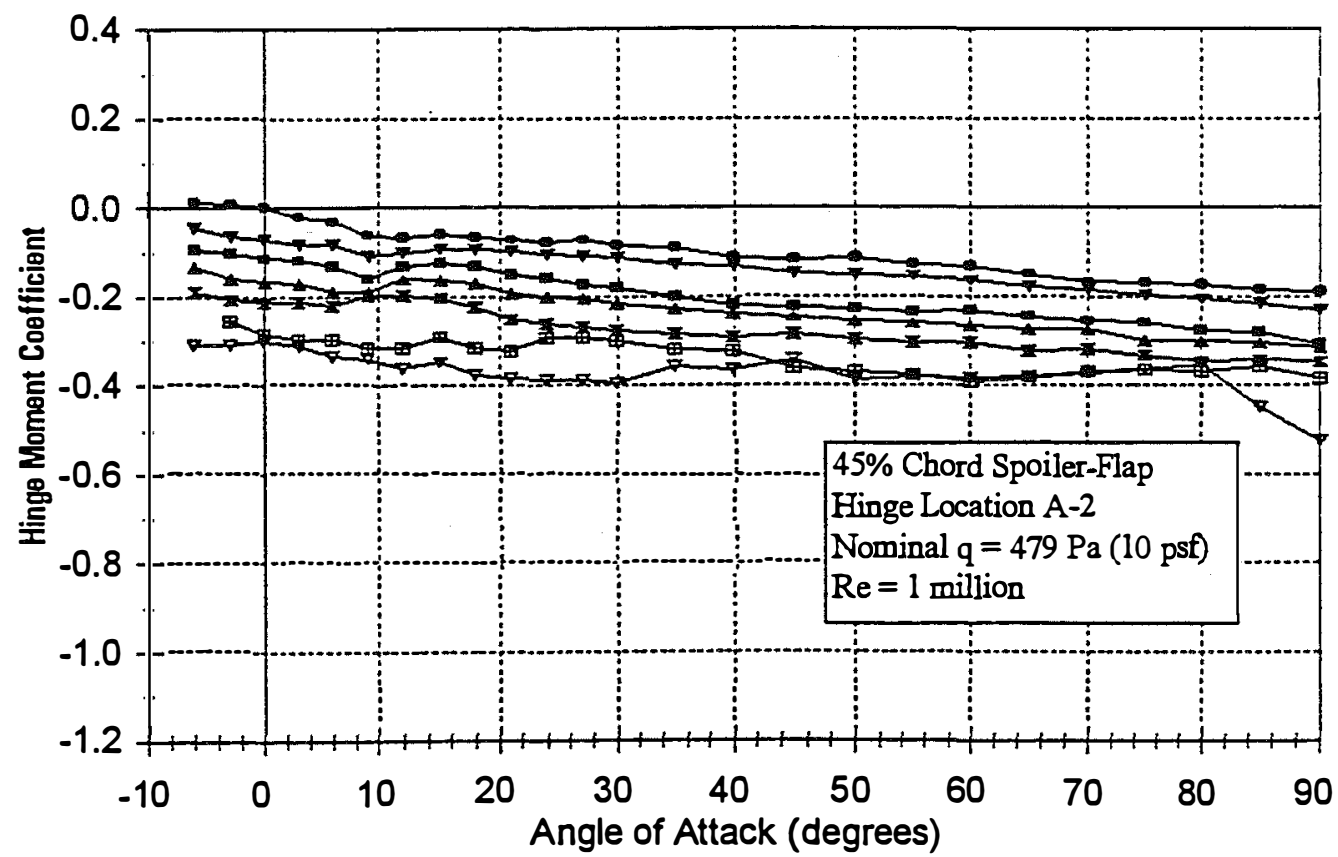

$$
\begin{aligned}
& \rightarrow \text { Delta }=0 \rightarrow \text { Delta }=15 \rightarrow \text { Delta }=30 \rightarrow \text { Delta }=45 \\
& \rightarrow \text { Delta }=60 \rightarrow \text { Delta }=75 \rightarrow \text { Delta }=90
\end{aligned}
$$

Figure A-8d. Hinge Moment Data, $\delta-S w e e p$ for $45 \%$ Chord Spoiler-Flap, Hinge A-2 


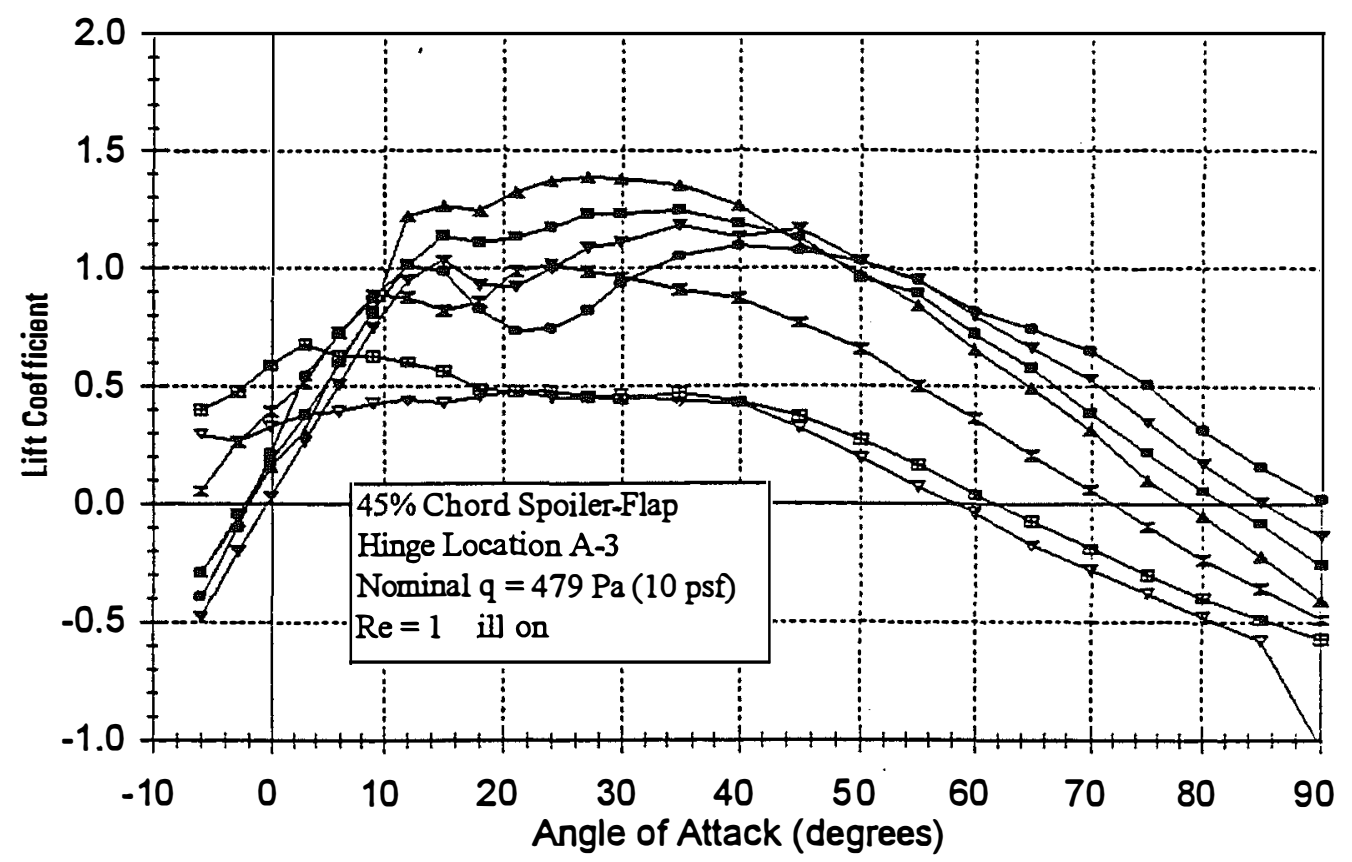

$$
\begin{aligned}
& \rightarrow \text { Delta }=0 \rightarrow \text { Delta }=15 \rightarrow \text { Delta }=30 \rightarrow \text { Delta }=45 \\
& \rightarrow \text { Delta }=60 \rightarrow \text { Delta }=75 \rightarrow \text { Delta }=90
\end{aligned}
$$

Figure A-9a. Lift Data, $\delta$-Sweep for $\mathbf{4 5} \%$ Chord Spoiler.Flap, Hinge A-3

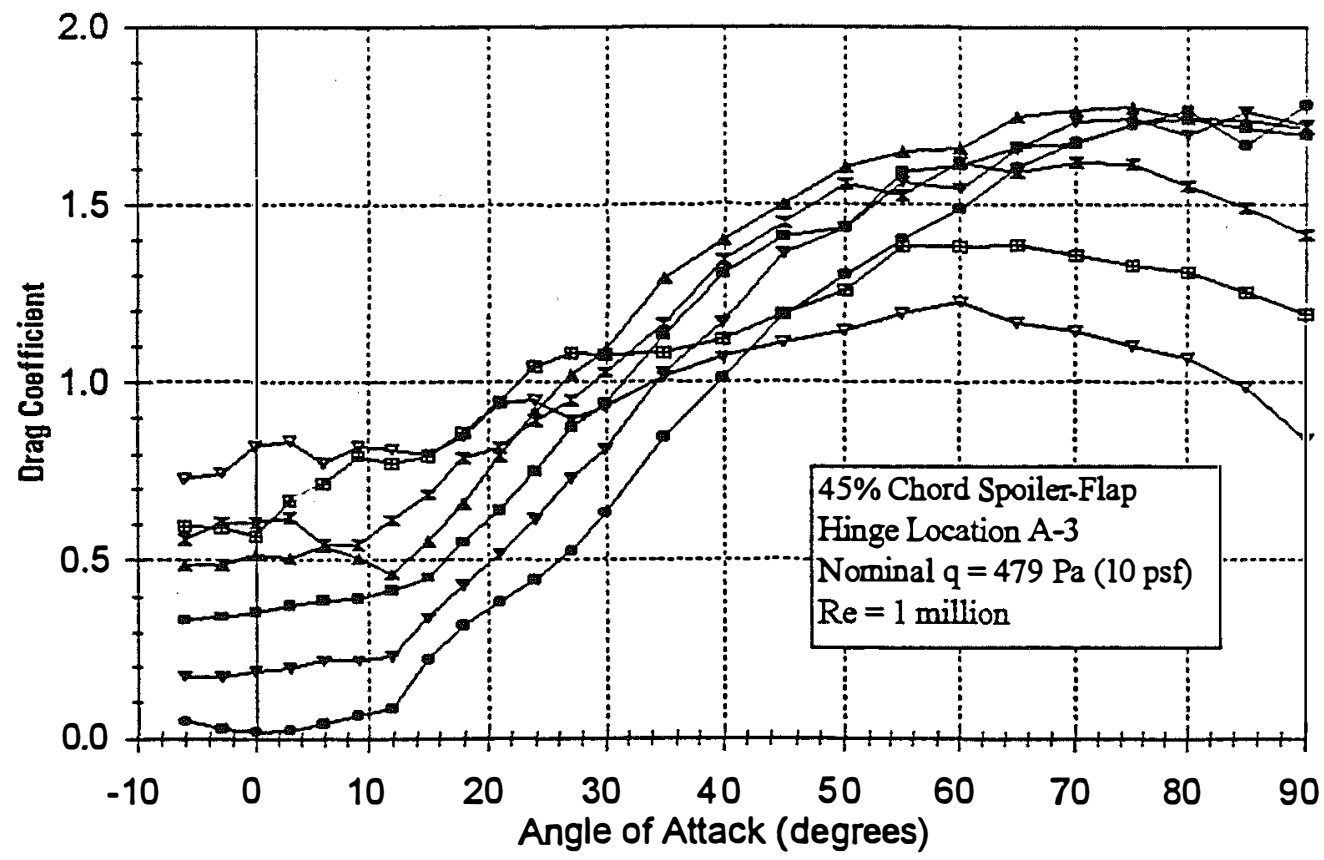

$$
\begin{aligned}
& \rightarrow \text { Delta }=0 \rightarrow \text { Delta }=15 \rightarrow \text { Delta }=30 \rightarrow \text { Delta }=45 \\
& \rightarrow \text { Delta }=60 \rightarrow \text { Delta }=75 \rightarrow \text { Delta }=90
\end{aligned}
$$

Figure A-9b. Drag Data, $\delta$-Sweep for $45 \%$ Chord Spoiler.Flap, Hinge A-3 


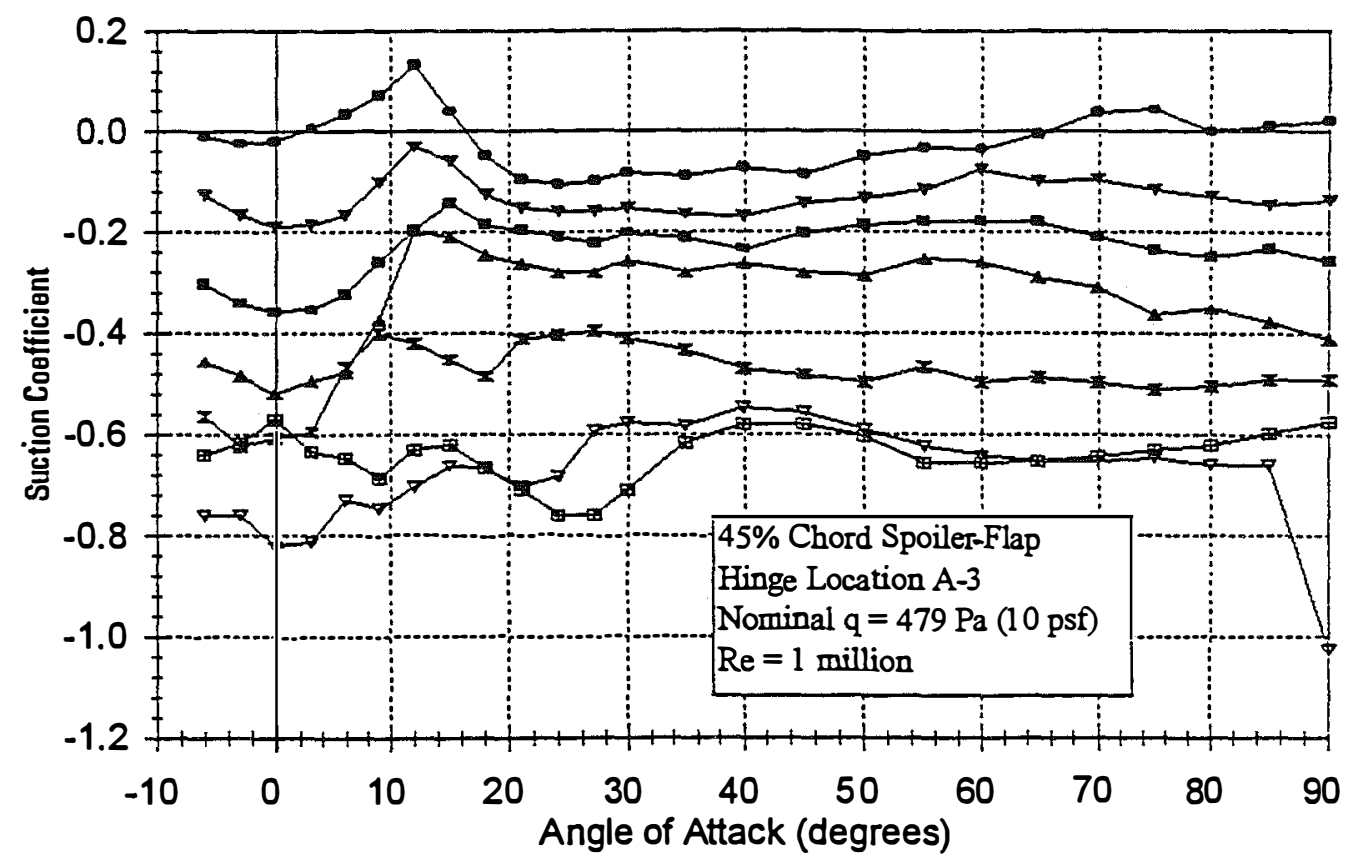

$$
\begin{aligned}
& \rightarrow \text { Delta }=0 \rightarrow \text { Delta }=15 \rightarrow \text { Delta }=30 \rightarrow \text { Delta }=45 \\
& \rightarrow \text { Delta }=60 \rightarrow \text { Delta }=75 \rightarrow \text { Delta }=90
\end{aligned}
$$

Figure A-9c. Suction Data, $\delta$ Sweep for $45 \%$ Chord Spoiler-Flap, Hinge A-3

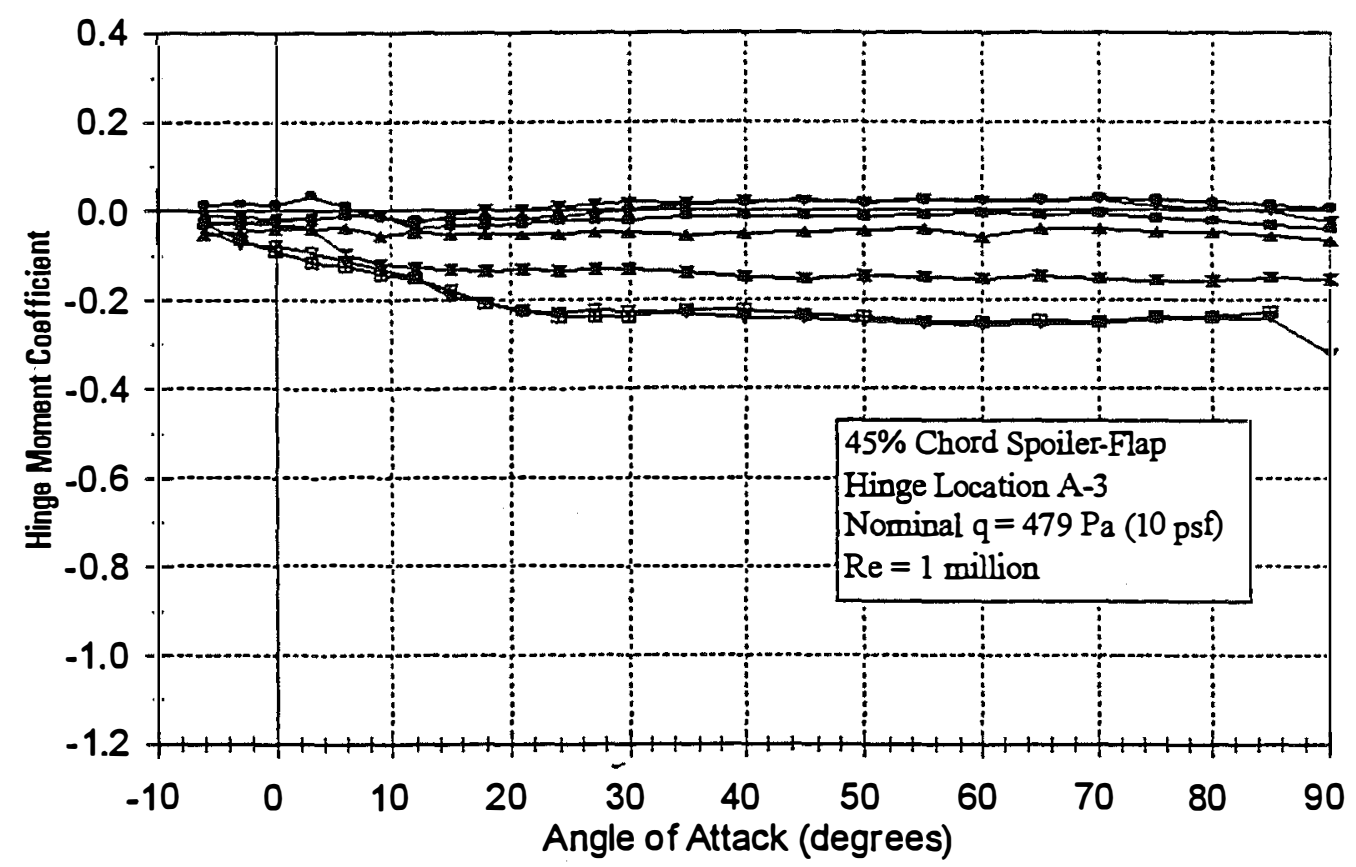

$$
\begin{aligned}
& \rightarrow \text { Delta }=0 \rightarrow \text { Delta }=15 \rightarrow \text { Delta }=30 \rightarrow \text { Delta }=45 \\
& \rightarrow \text { Delta }=60 \rightarrow \text { Delta }=75 \rightarrow \text { Delta }=90
\end{aligned}
$$

Figure A-9d. Hinge Moment Data, $\delta$ Sweep for $45 \%$ Chord Spoiler-Flap, Hinge A-3 


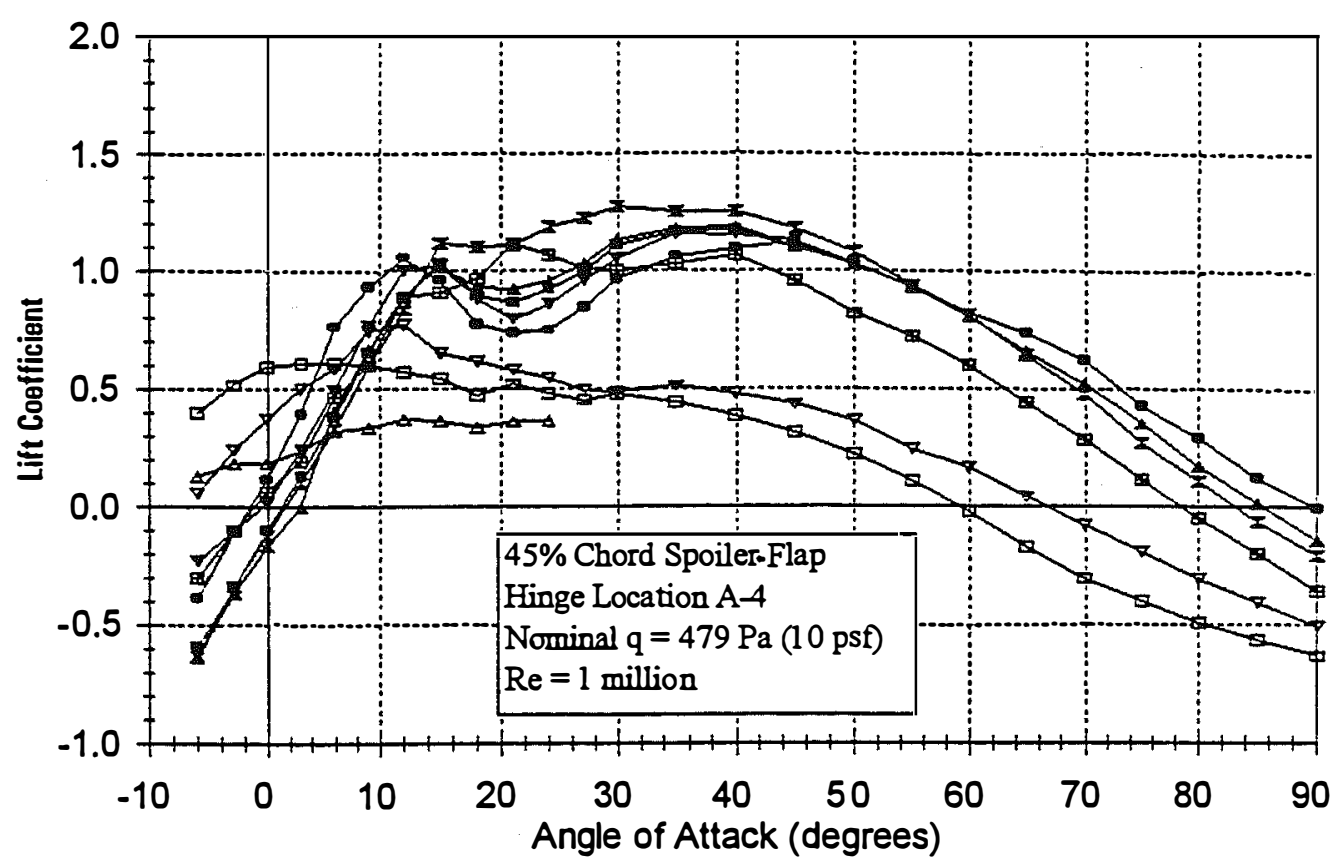

$$
\begin{aligned}
& \rightarrow \text { Delta }=0 \rightarrow \text { Delta }=5 \rightarrow \text { Delta }=10 \rightarrow \text { Delta }=15 \rightarrow \text { Delta }=30 \\
& \rightarrow \text { Delta }=45 \rightarrow \text { Delta }=60 \rightarrow \text { Delta }=75 \rightarrow \text { Delta }=90
\end{aligned}
$$

Figure A-10a. Lift Data, $\delta$-Sweep for $45 \%$ Chord Spoiler-Flap, Hinge A-4

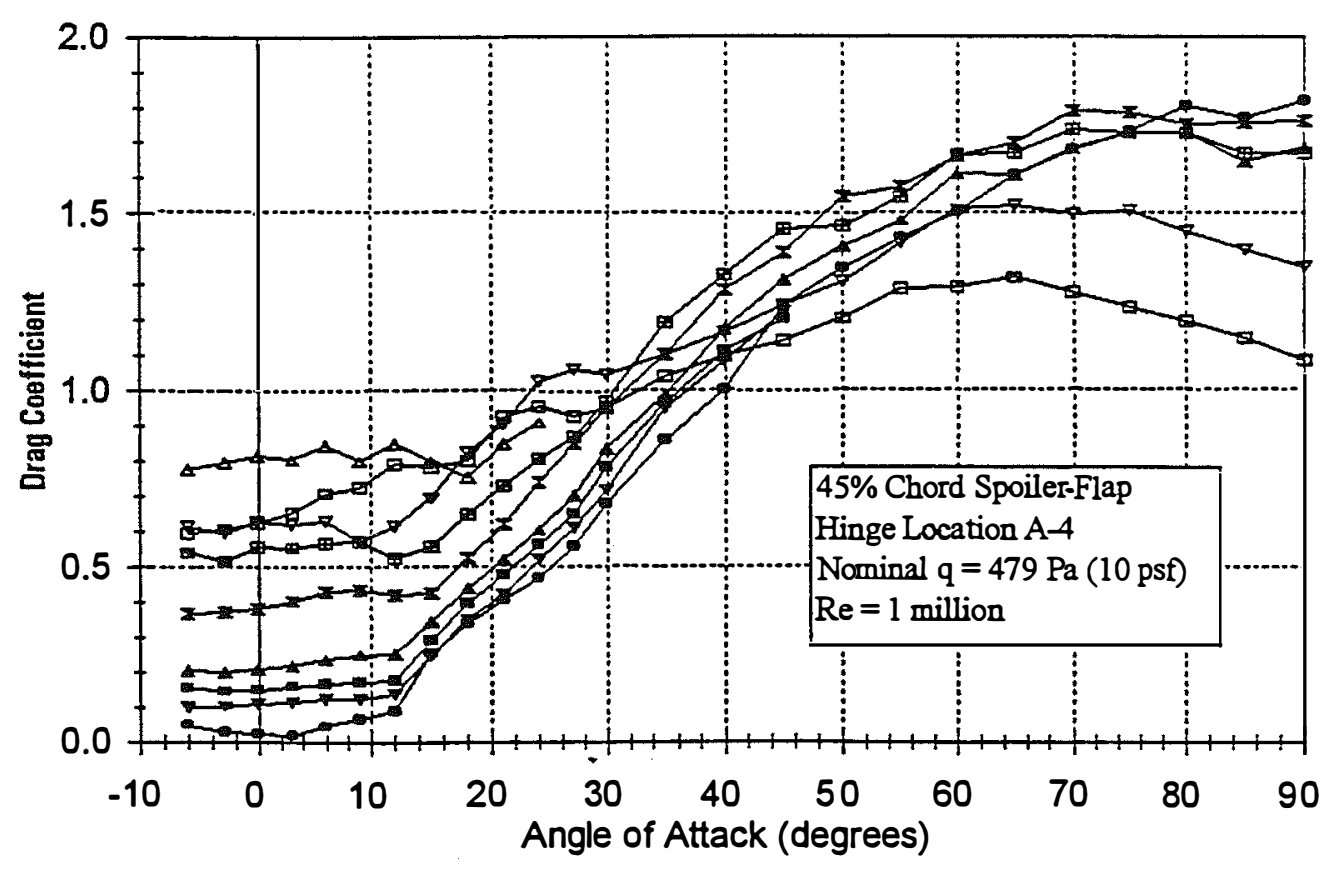

$$
\begin{aligned}
& \rightarrow \text { Delta }=0 \rightarrow \text { Delta }=5 \rightarrow \text { Delta }=10 \rightarrow \text { Delta }=15 \rightarrow \text { Delta }=30 \\
& \rightarrow \text { Delta }=45 \rightarrow \text { Delta }=60 \rightarrow \text { Delta }=75 \rightarrow \text { Delta }=90
\end{aligned}
$$

Figure A-10b. Drag Data, $\delta-S w e e p$ for $45 \%$ Chord Spoiler-Flap, Hinge A-4 


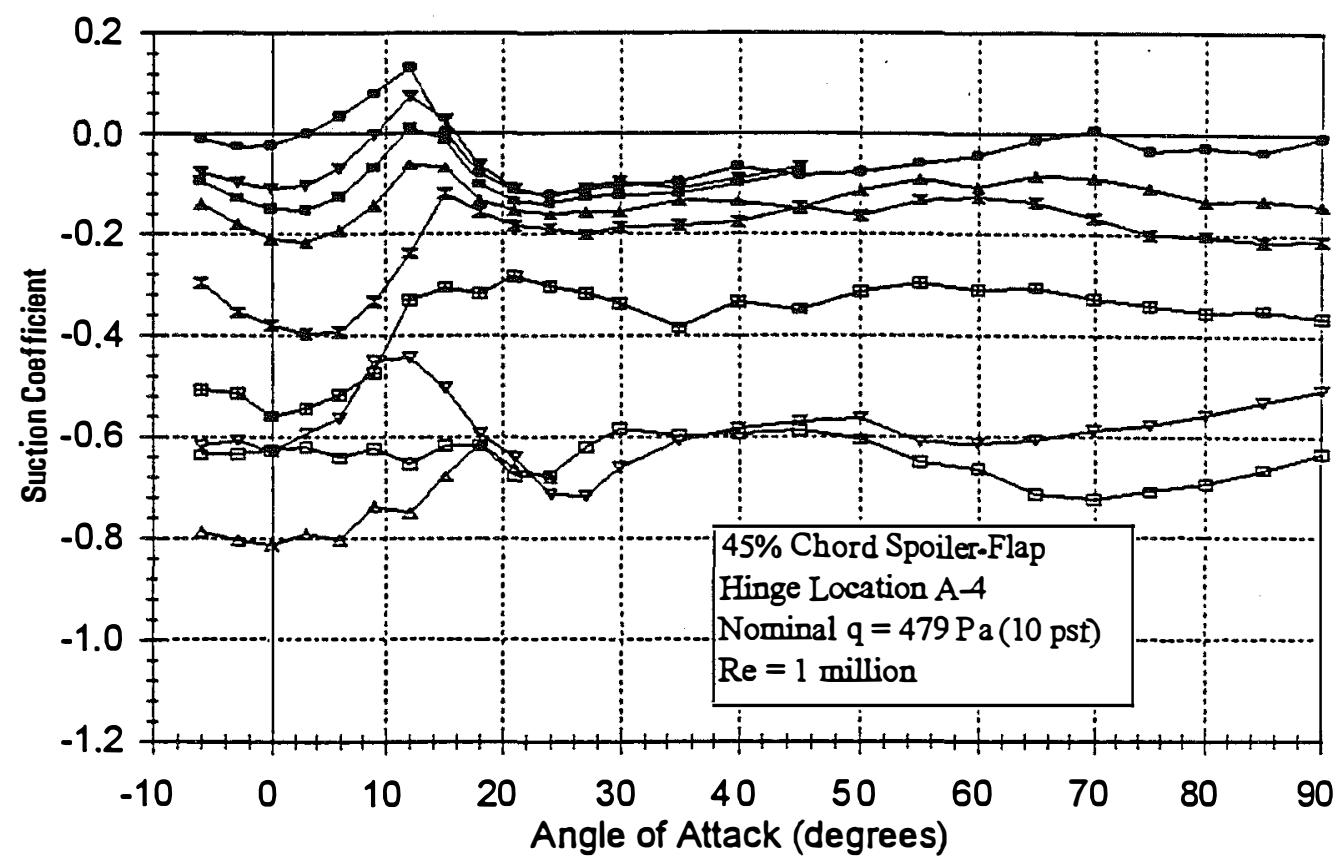

$\rightarrow$ Delta $=0 \rightarrow$ Delta $=5 \rightarrow$ Delta $=10 \rightarrow$ Delta $=15 \rightarrow$ Delta $=30$
$\rightarrow$ Delta $=45 \rightarrow$ Delta $=60 \rightarrow$ Delta $=75 \rightarrow$ Delta $=90$

Figure A-10c. Suction Data, $\delta$-Sweep for $\mathbf{4 5} \%$ Chord Spoiler-Flap, Hinge A-4

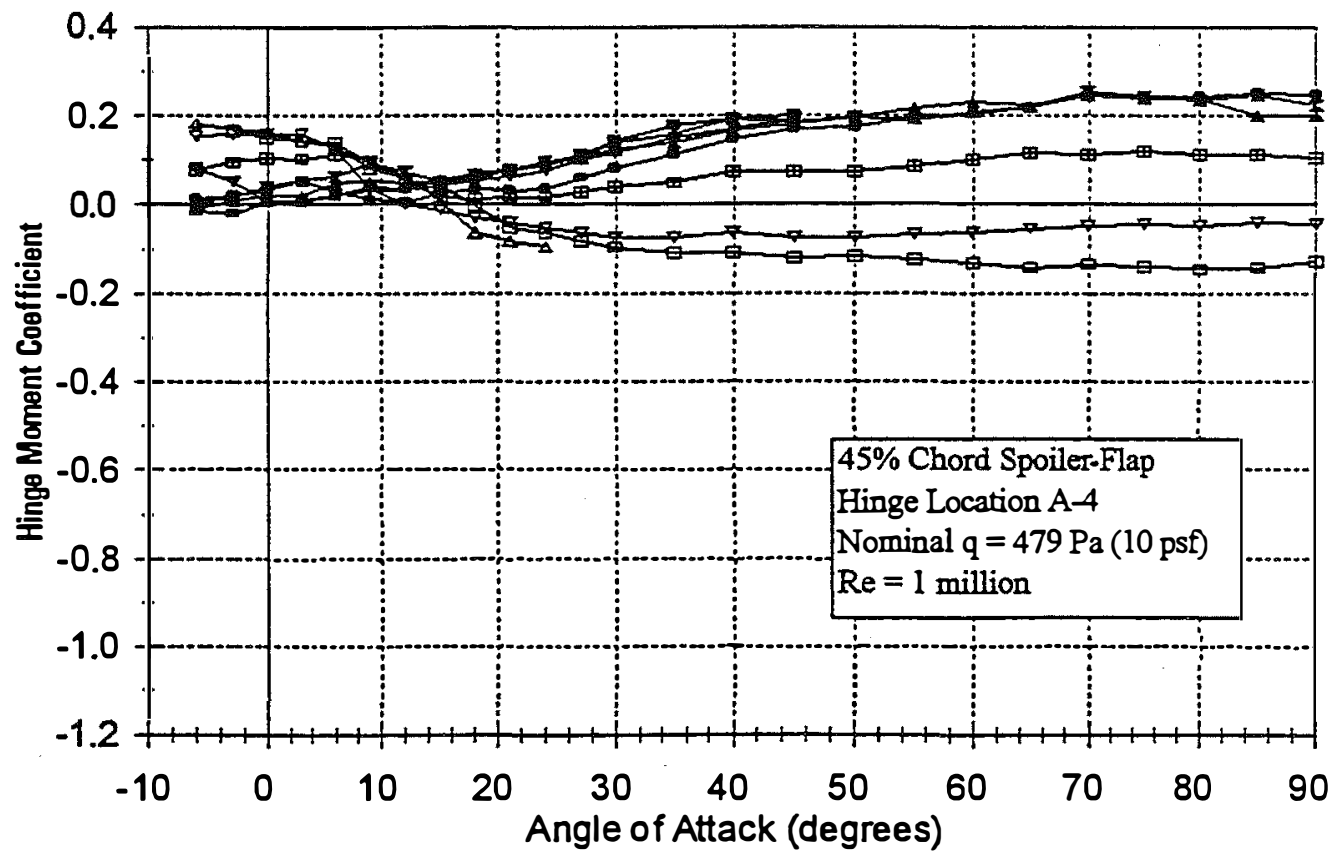

$$
\begin{aligned}
& \rightarrow \text { Delta }=0 \rightarrow \text { Delta }=5 \rightarrow \text { Delta }=10 \rightarrow \text { Delta }=15 \rightarrow \text { Delta }=30 \\
& \rightarrow \text { Delta }=45 \rightarrow \text { Delta }=60 \rightarrow \text { Delta }=75 \rightarrow \text { Delta }=90
\end{aligned}
$$

Figure A-10d. Hinge Moment Data, $\delta$-Sweep for $45 \%$ Chord Spoiler-Flap, Hinge A-4 


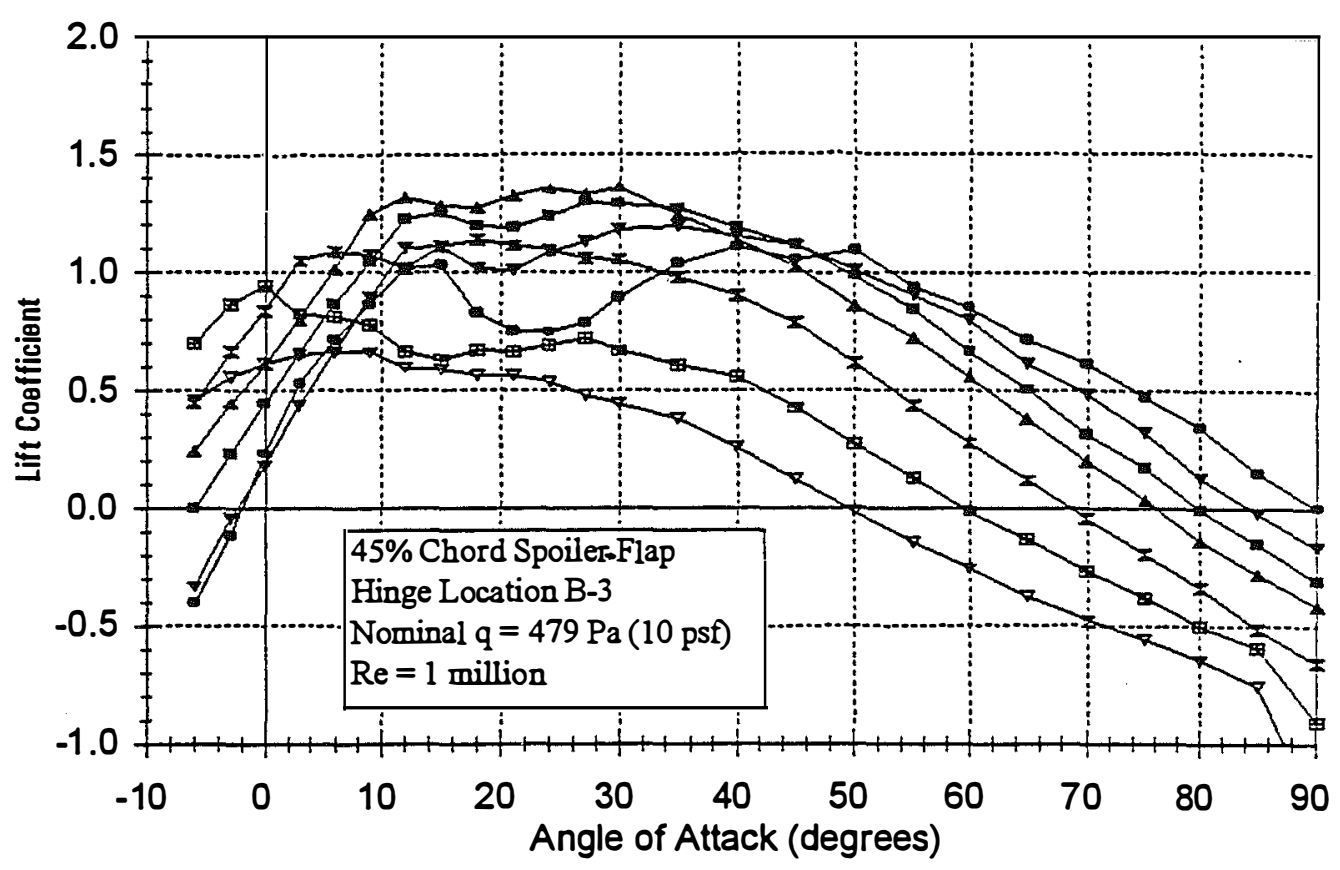

$$
\begin{aligned}
& \rightarrow \text { Delta }=0 \rightarrow \text { Delta }=15 \rightarrow \text { Delta }=30 \rightarrow \text { Delta }=45 \\
& \rightarrow \text { Delta }=60 \rightarrow \text { Delta }=75 \rightarrow \text { Delta }=90
\end{aligned}
$$

Figure A-11a. Lift Data, $\delta$-Sweep for $45 \%$ Chord Spoiler-Flap, Hinge B-3

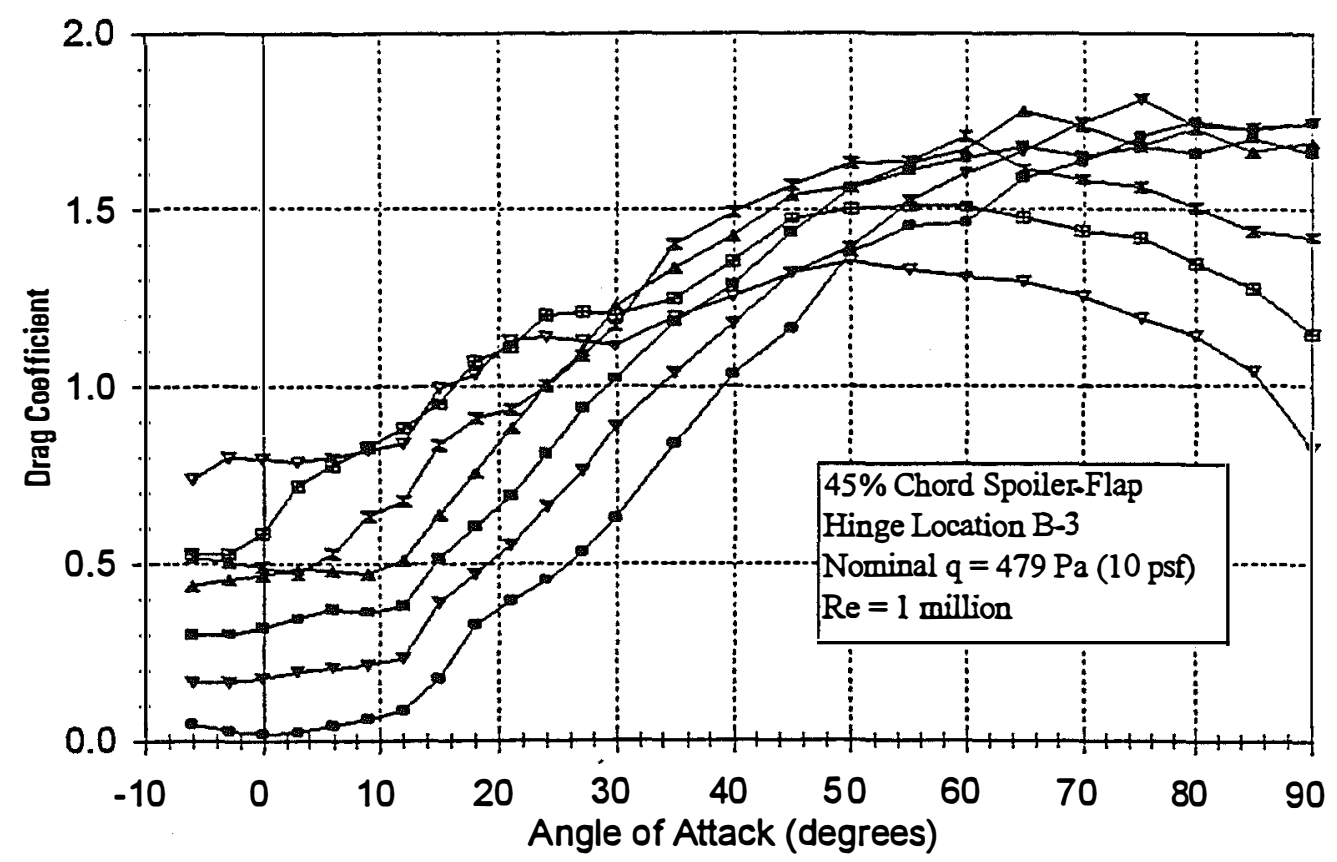

$$
\begin{aligned}
& \rightarrow \text { Delta }=0 \rightarrow \text { Delta }=15 \rightarrow \text { Delta }=30 \rightarrow \text { Delta }=45 \\
& \rightarrow \text { Delta }=60 \rightarrow \text { Delta }=75 \rightarrow \text { Delta }=90
\end{aligned}
$$

Figure A-11b. Drag Data, $\delta-S w e e p$ for $45 \%$ Chord Spoiler-Flap, Hinge B-3 


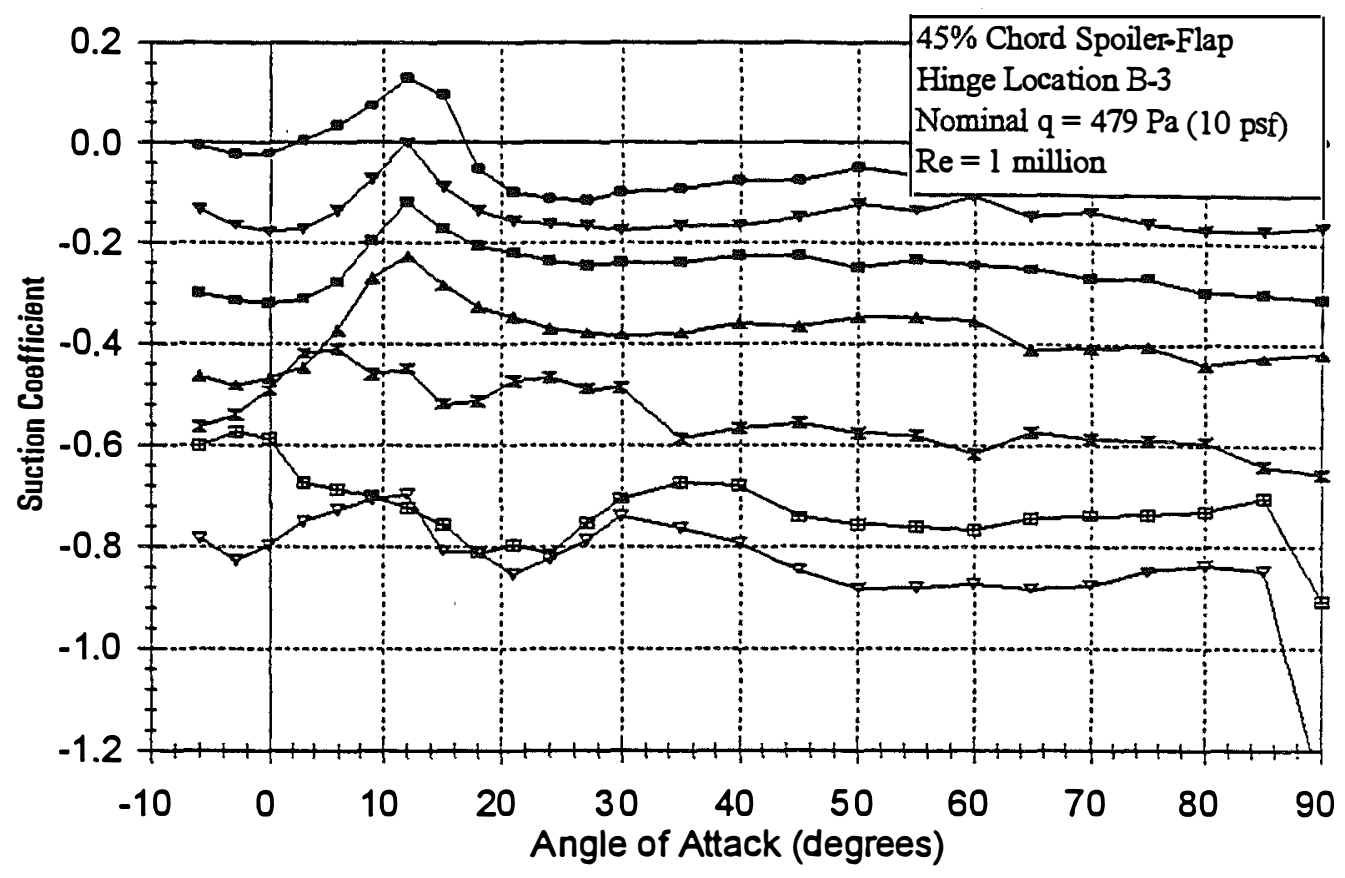

$$
\begin{aligned}
& \rightarrow \text { Delta }=0 \rightarrow \text { Delta }=15 \rightarrow \text { Delta }=30 \rightarrow \text { Delta }=45 \\
& \rightarrow \text { Delta }=60 \rightarrow \text { Delta }=75 \rightarrow \text { Delta }=90
\end{aligned}
$$

Figure A-11c. Suction Data, $\delta$-Sweep for $45 \%$ Chord Spoiler-Flap, Hinge B-3

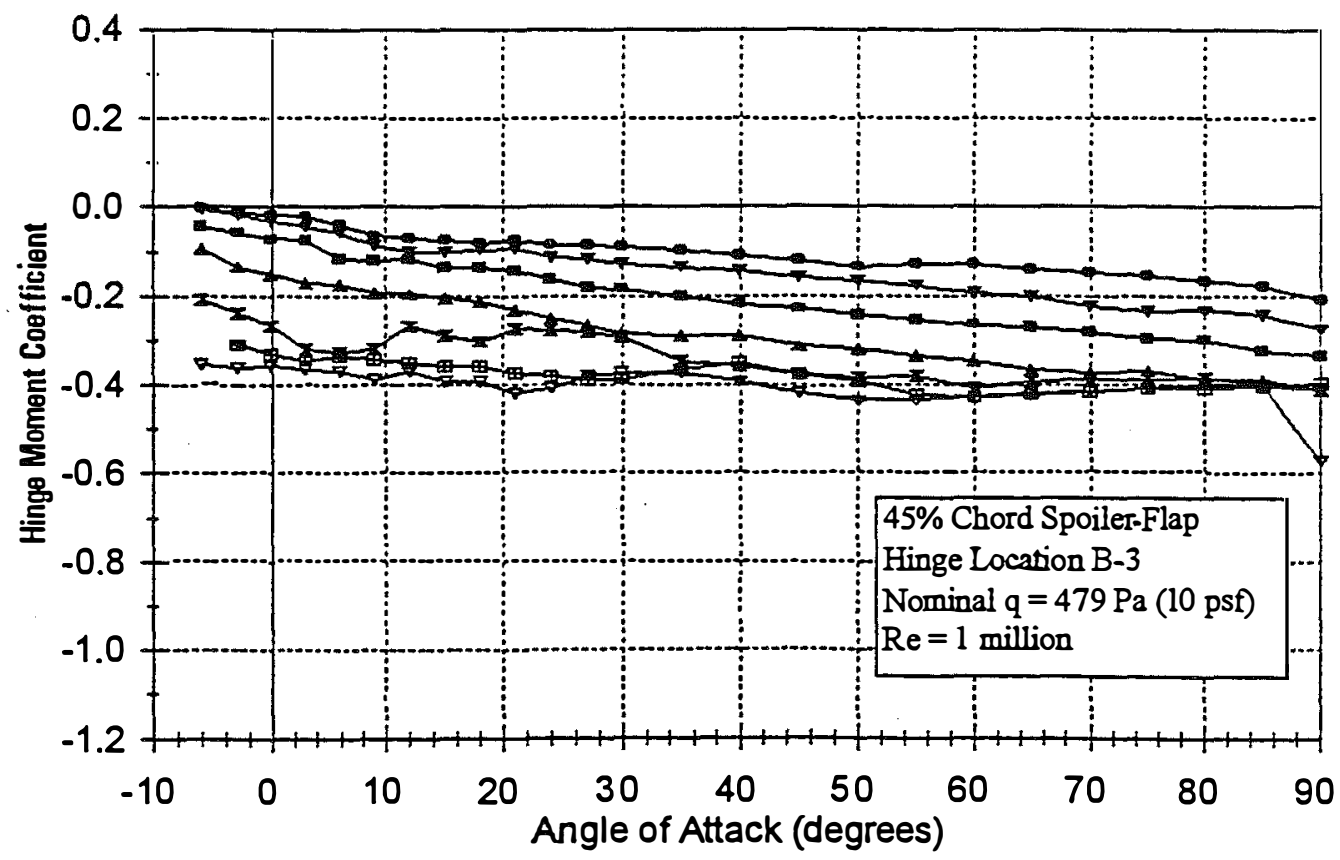

$$
\begin{aligned}
& \rightarrow \text { Delta }=0 \rightarrow \text { Delta }=15 \rightarrow \text { Delta }=30 \rightarrow \text { Delta }=45 \\
& \rightarrow \text { Delta }=60 \rightarrow \text { Delta }=75 \rightarrow \text { Delta }=90
\end{aligned}
$$

Figure A-11d. Hinge Moment Data, $\delta$-Sweep for $45 \%$ Chord Spoiler-Flap, Hinge B-3 


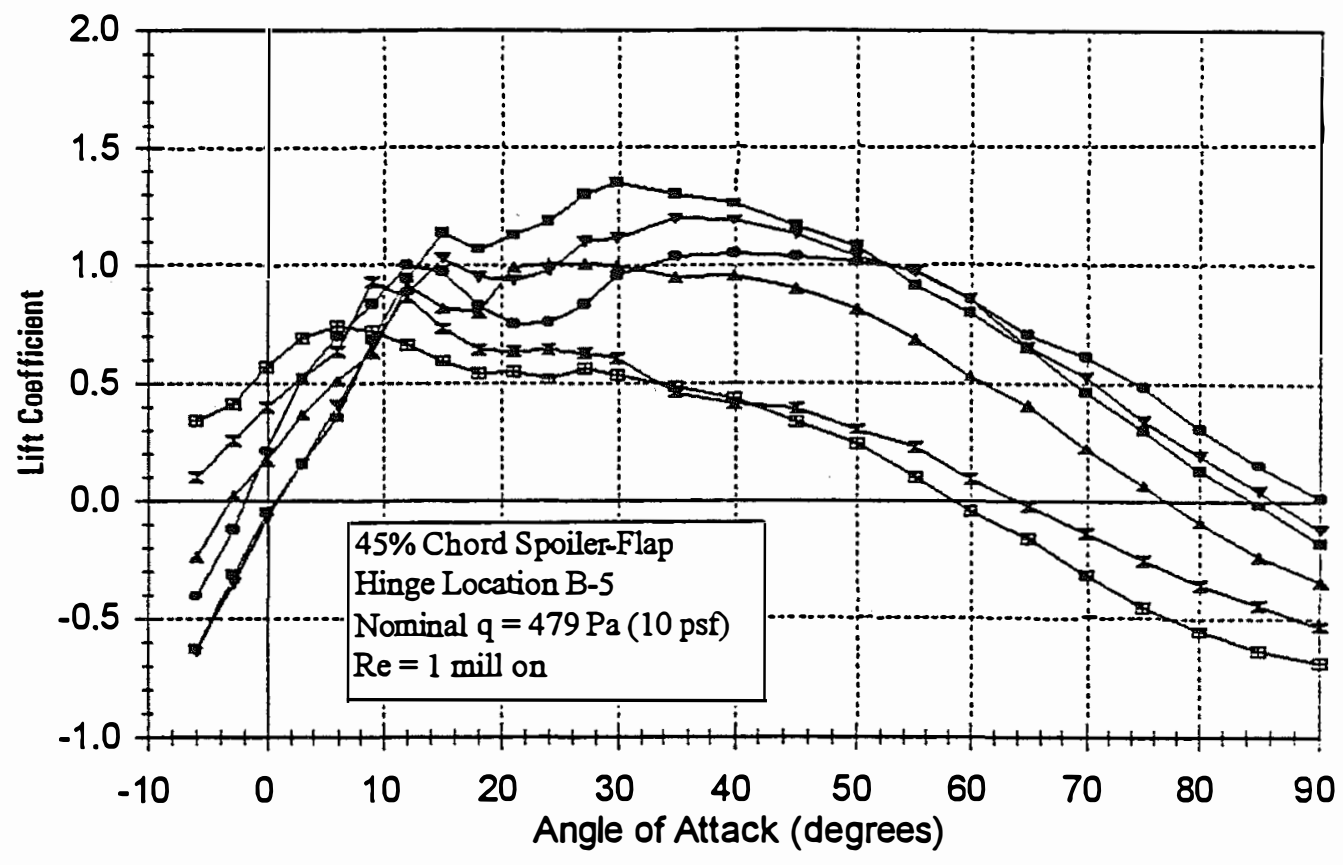

$$
\begin{aligned}
& \longrightarrow \text { Delta }=0 \rightarrow \text { Delta }=15 \rightarrow \text { Delta }=30 \\
& \longrightarrow \text { Delta }=45 \rightarrow \text { Delta }=60 \rightarrow \text { Delta }=75
\end{aligned}
$$

Figure A-12a. Lift Data, $\delta$-Sweep for $45 \%$ Chord Spoiler-Flap, Hinge B-5

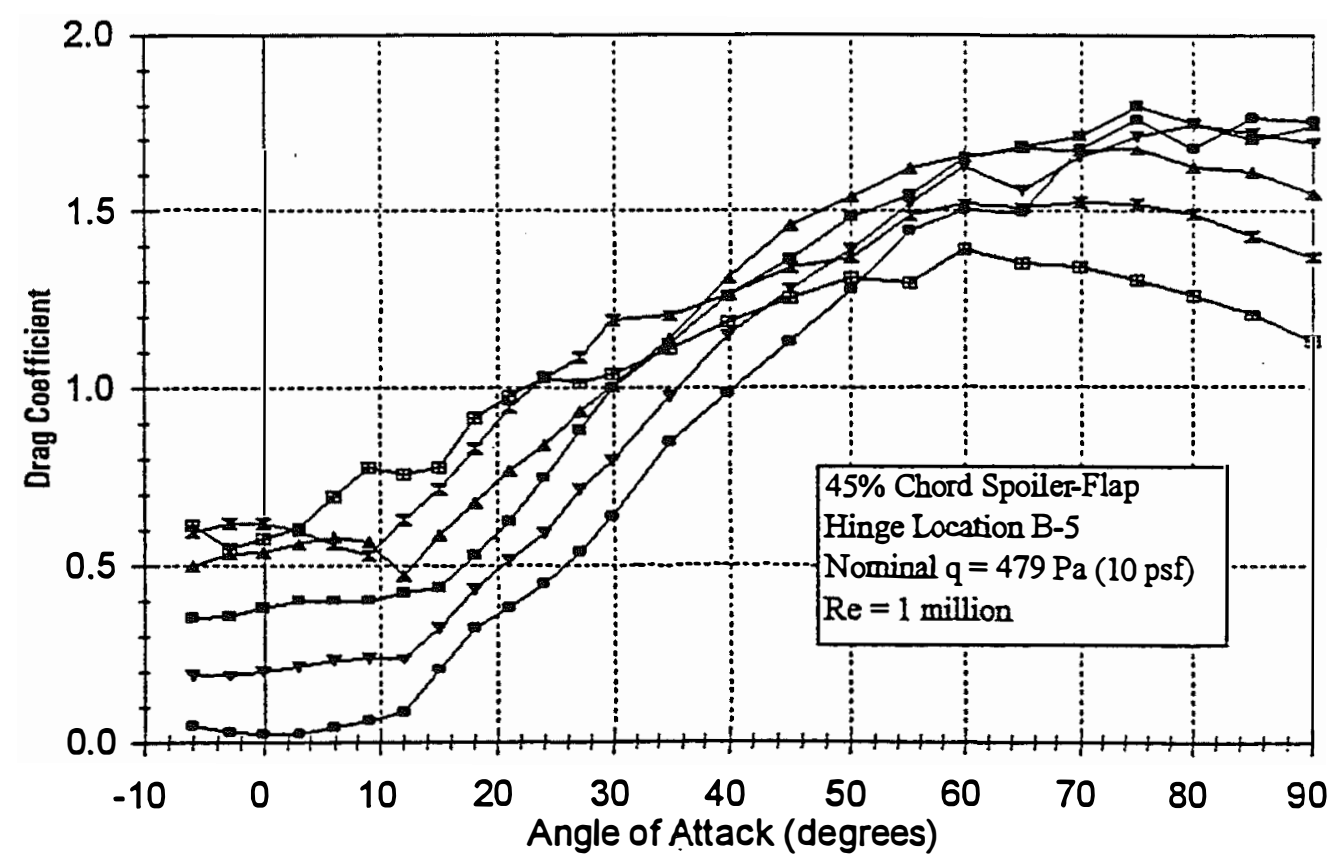

$$
\begin{aligned}
& \rightarrow \text { Delta }=0 \rightarrow \text { Delta }=15 \rightarrow \text { Delta }=30 \\
& \rightarrow \text { Delta }=45 \rightarrow \text { Delta }=60 \rightarrow \text { Delta }=75
\end{aligned}
$$

Figure A-12b. Drag Data, $\delta$-Sweep for $45 \%$ Chord Spoiler-Flap, Hinge B-5 


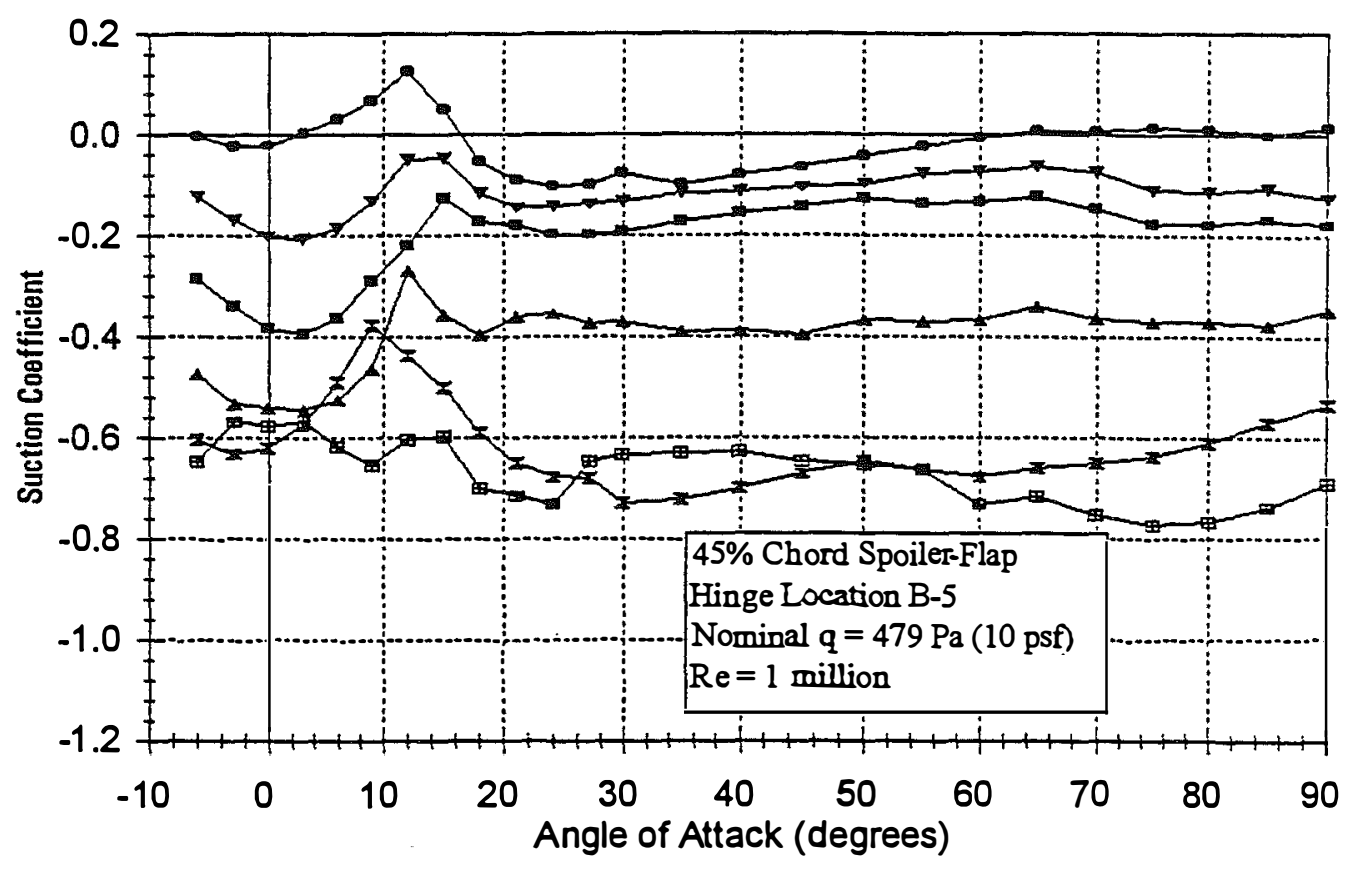

$$
\begin{aligned}
& \rightarrow \text { Delta }=0 \rightarrow \text { Delta }=15 \rightarrow \text { Delta }=30 \\
& - \text { Delta }=45 \rightarrow \text { Delta }=60 \rightarrow \text { Delta }=75
\end{aligned}
$$

Figure A-12c. Suction Data, $\delta$-Sweep for $45 \%$ Chord SpoilerFlap, Hinge B-5

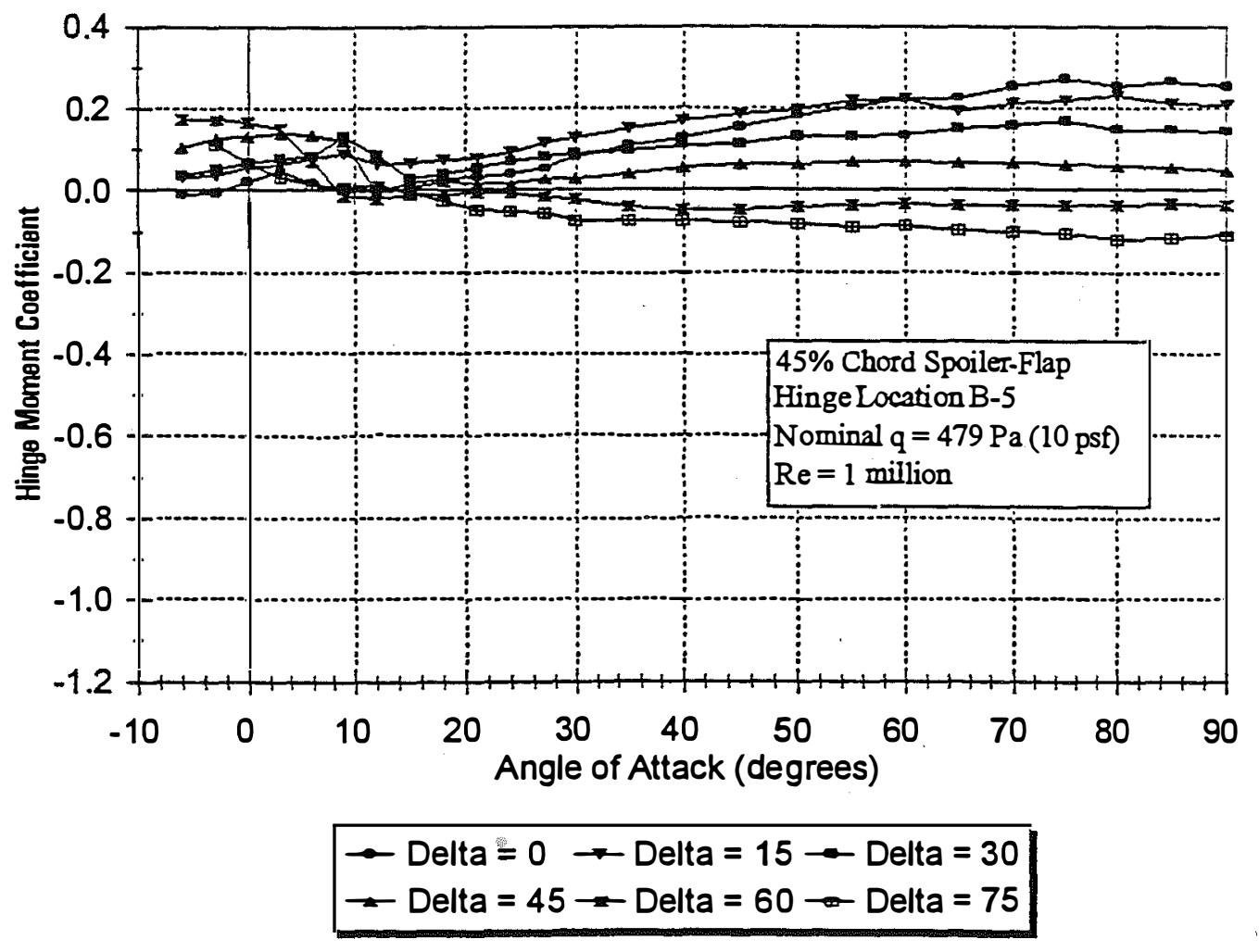

Figure A-12d. Hinge Moment Data, $\delta$-Sweep for $45 \%$ Chord Spoiler-Flap, Hinge B-5 


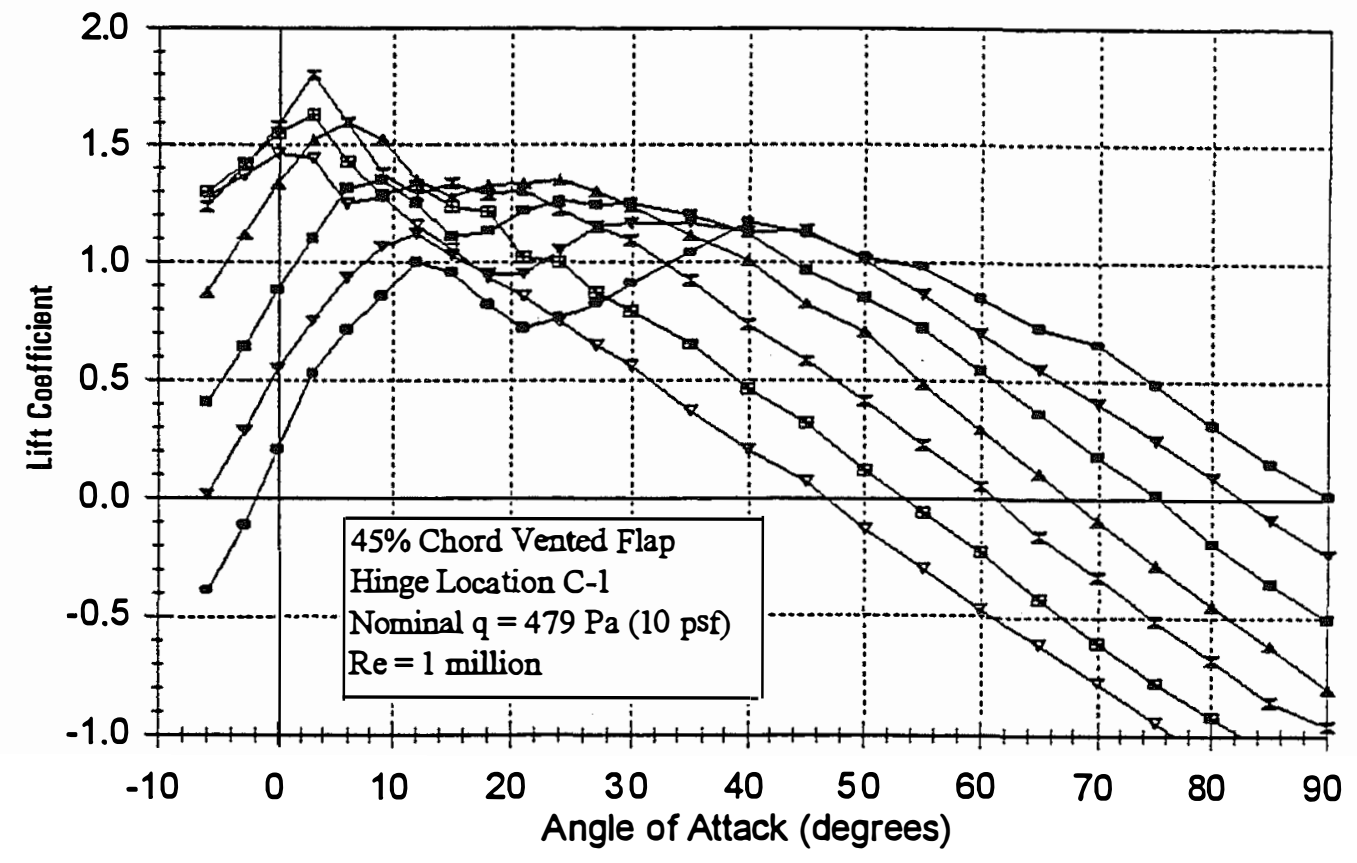

$$
\begin{aligned}
& \rightarrow \text { Delta }=0 \rightarrow \text { Delta }=15 \rightarrow \text { Delta }=30 \rightarrow \text { Delta }=45 \\
& \rightarrow \text { Delta }=60 \rightarrow \text { Delta }=75 \rightarrow \text { Delta }=90
\end{aligned}
$$

Figure A-13a. Lift Data, $\delta$ Sweep for $45 \%$ Chord Vented Flap, Hinge C-1

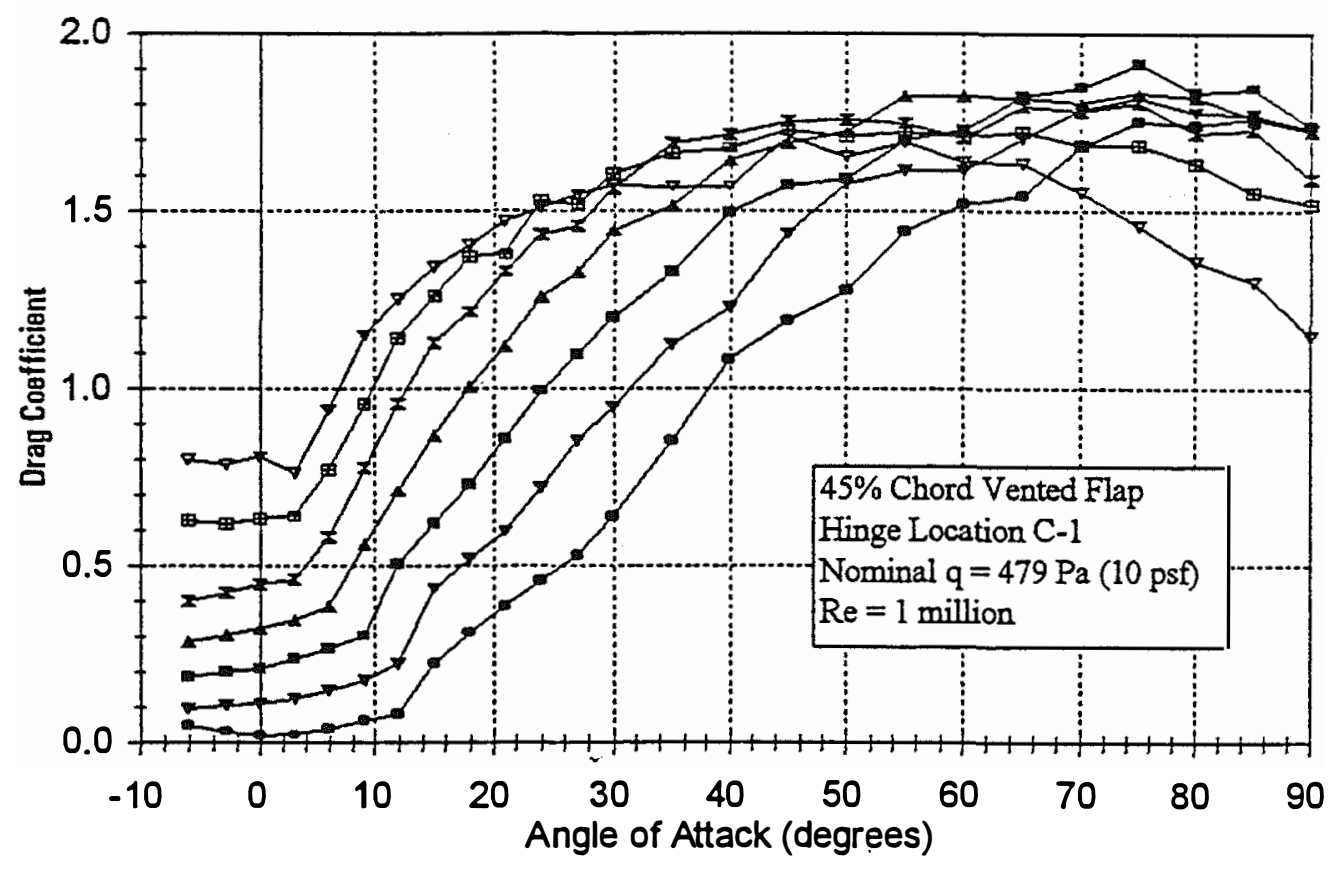

$$
\begin{aligned}
& \rightarrow \text { Delta }=0 \rightarrow \text { Delta }=15 \rightarrow \text { Delta }=30 \rightarrow \text { Delta }=45 \\
& \rightarrow \text { Delta }=60 \rightarrow \text { Delta }=75 \rightarrow \text { Delta }=90
\end{aligned}
$$

Figure A-13b. Drag Data, $\delta$ Sweep for $\mathbf{4 5 \%}$ Chord Vented Flap, Hinge C-1 


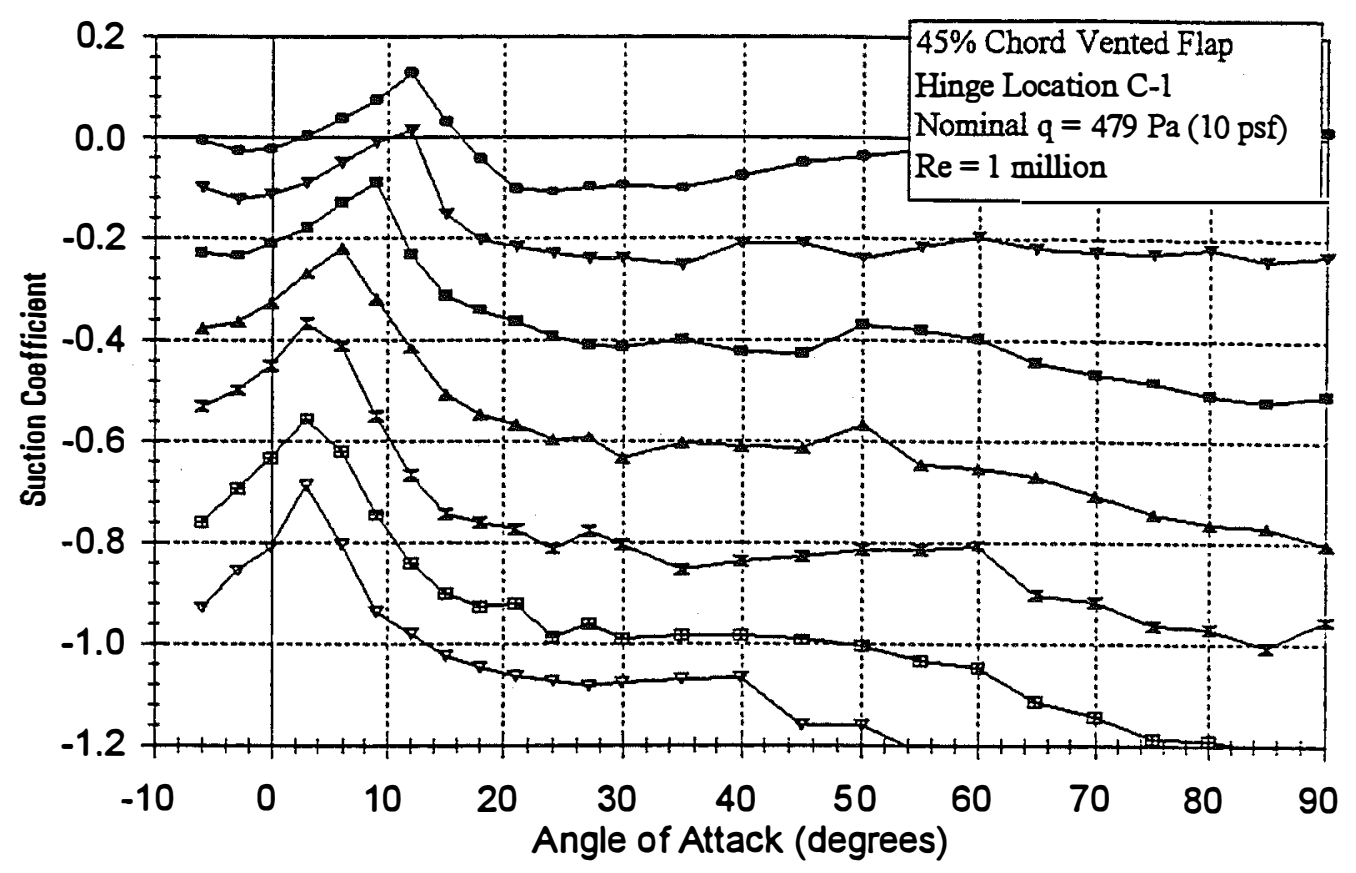

$$
\begin{aligned}
& \rightarrow \text { Delta }=0 \rightarrow \text { Delta }=15 \rightarrow \text { Delta }=30 \rightarrow \text { Delta }=45 \\
& \rightarrow \text { Delta }=60 \rightarrow \text { Delta }=75 \rightarrow \text { Delta }=90
\end{aligned}
$$

Figure A-13c. Suction Data, §Sweep for $45 \%$ Chord Vented Flap, Hinge C-1

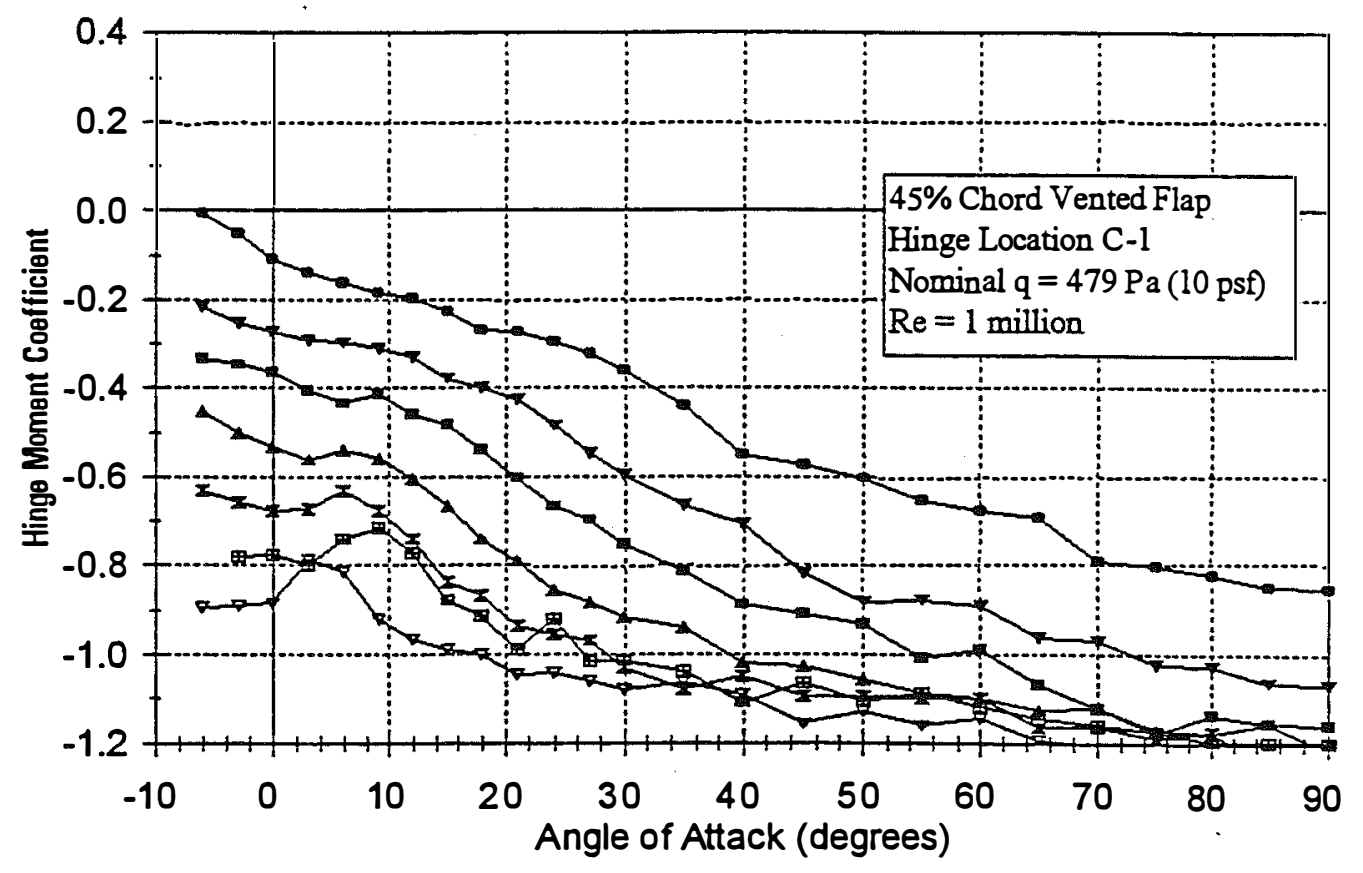

$$
\begin{aligned}
& \rightarrow \text { Delta }=0 \rightarrow \text { Delta }=15 \rightarrow \text { Delta }=30 \rightarrow \text { Delta }=45 \\
& \rightarrow \text { Delta }=60 \rightarrow \text { Delta }=75 \rightarrow \text { Delta }=90
\end{aligned}
$$

Figure A-13d. Hinge Moment Data, $\delta$-Sweep for $45 \%$ Chord Vented Flap, Hinge C-1 


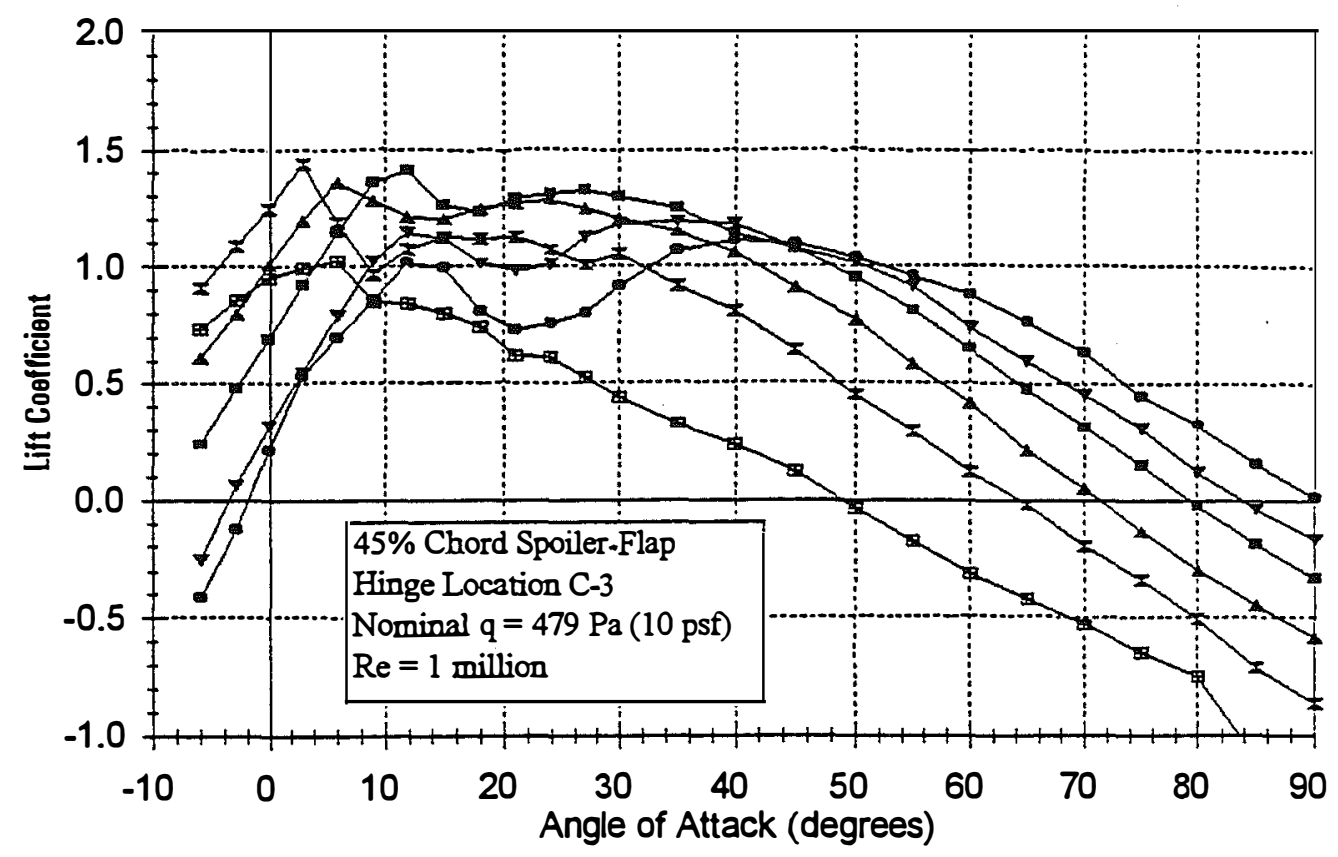

$$
\begin{aligned}
& \rightarrow \text { Delta }=0 \rightarrow \text { Delta }=15 \rightarrow \text { Delta }=30 \\
& \rightarrow \text { Delta }=45 \rightarrow \text { Delta }=60 \rightarrow \text { Delta }=85
\end{aligned}
$$

Figure A-14a. Lift Data, $\delta$-Sweep for $45 \%$ Chord Spoiler-Flap, Hinge C-3

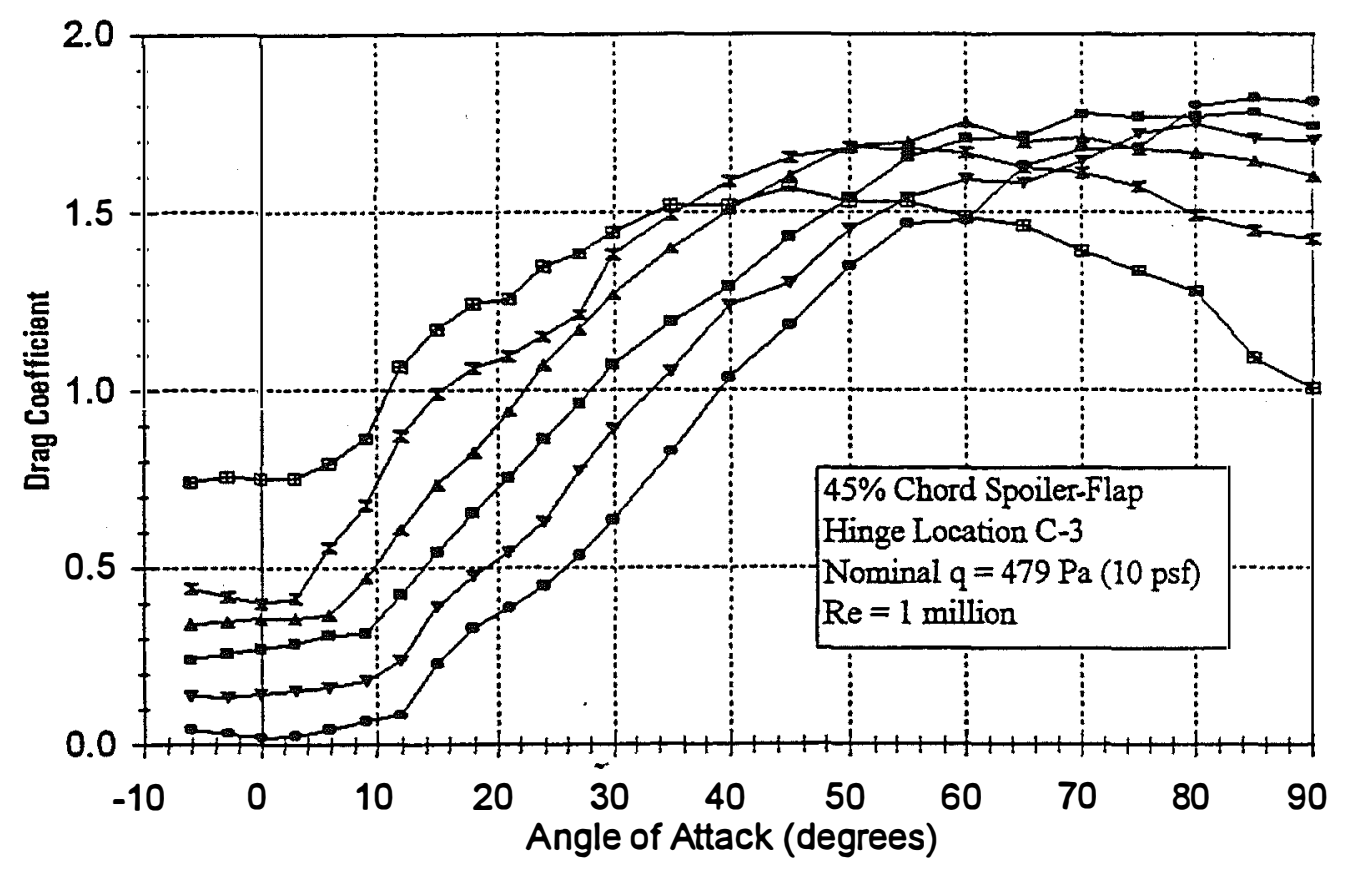$$
\rightarrow \text { Delta }=0 \rightarrow \text { Delta }=15 \rightarrow \text { Delta }=30
$$$$
\rightarrow \text { Delta }=45 \rightarrow \text { Delta }=60 \rightarrow \text { Delta }=85
$$

Figure A-14b. Drag Data, $\delta$-Sweep for $45 \%$ Chord Spoiler.Flap, Hinge C-3 


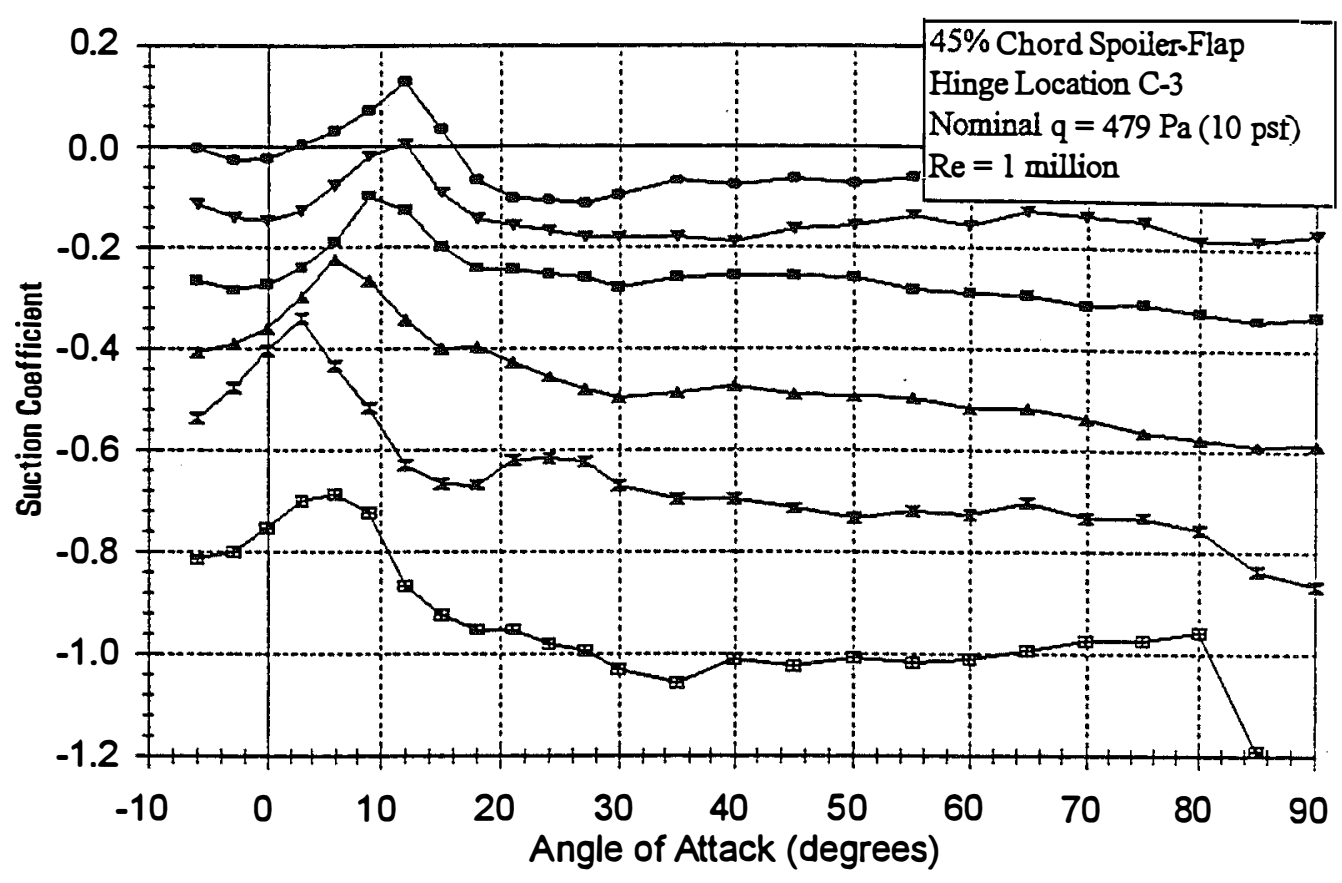

$$
\begin{aligned}
& \rightarrow \text { Delta }=0 \rightarrow \text { Delta }=15 \rightarrow \text { Delta }=30 \\
& - \text { Delta }=45 \rightarrow \text { Delta }=60 \rightarrow \text { Delta }=85
\end{aligned}
$$

Figure A-14c. Suction Data, $\delta$-Sweep for $45 \%$ Chord Spoiler-Flap, Hinge C-3

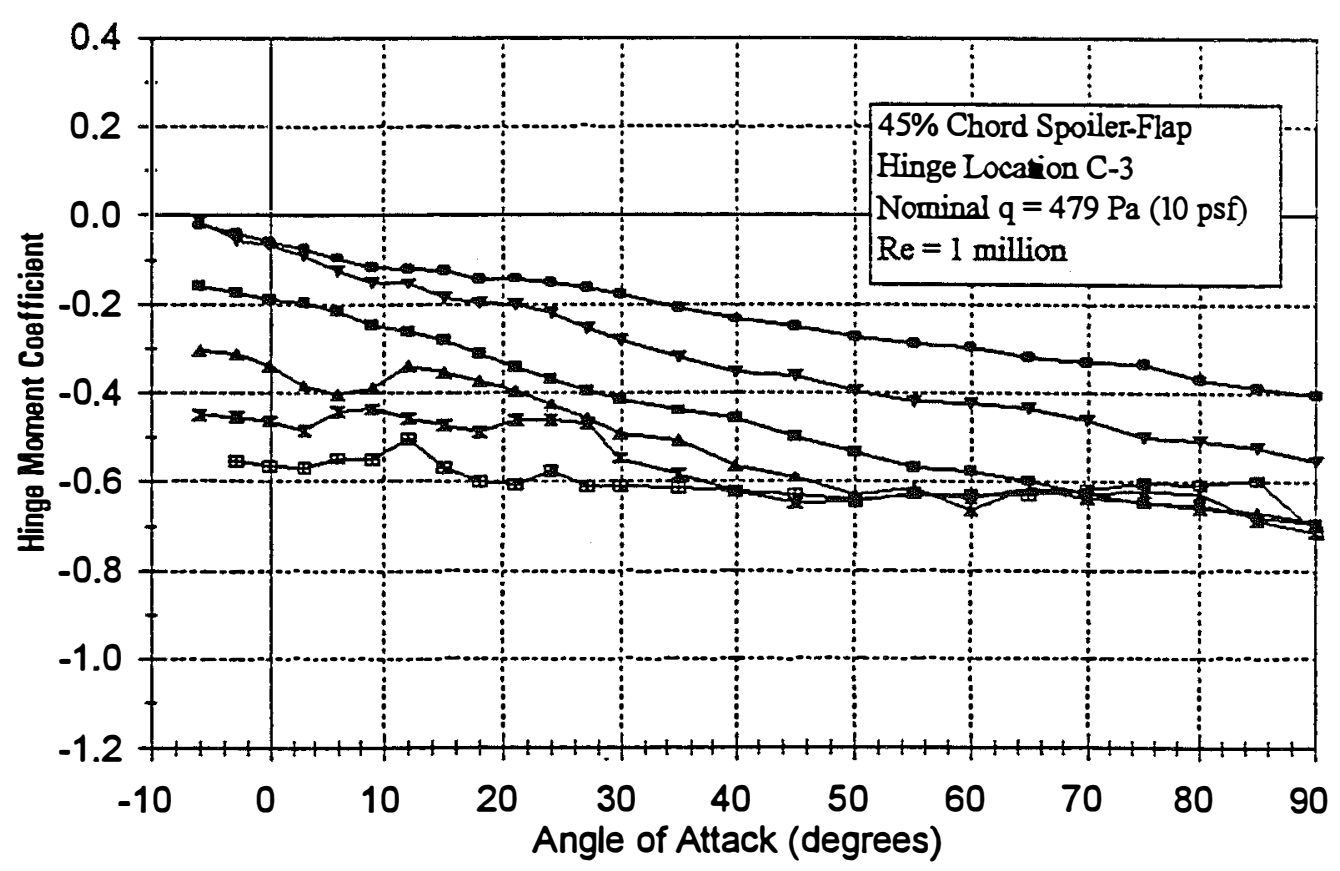

$$
\begin{aligned}
& \rightarrow \text { Delta }=0 \rightarrow \text { Delta }=15 \rightarrow \text { Delta }=30 \\
& - \text { Delta }=45 \rightarrow \text { Delta }=60 \rightarrow \text { Delta }=85
\end{aligned}
$$

Figure A-14d. Hinge Moment Data, $\delta$-Sweep for $45 \%$ Chord Spoiler-Flap, Hinge C-3 


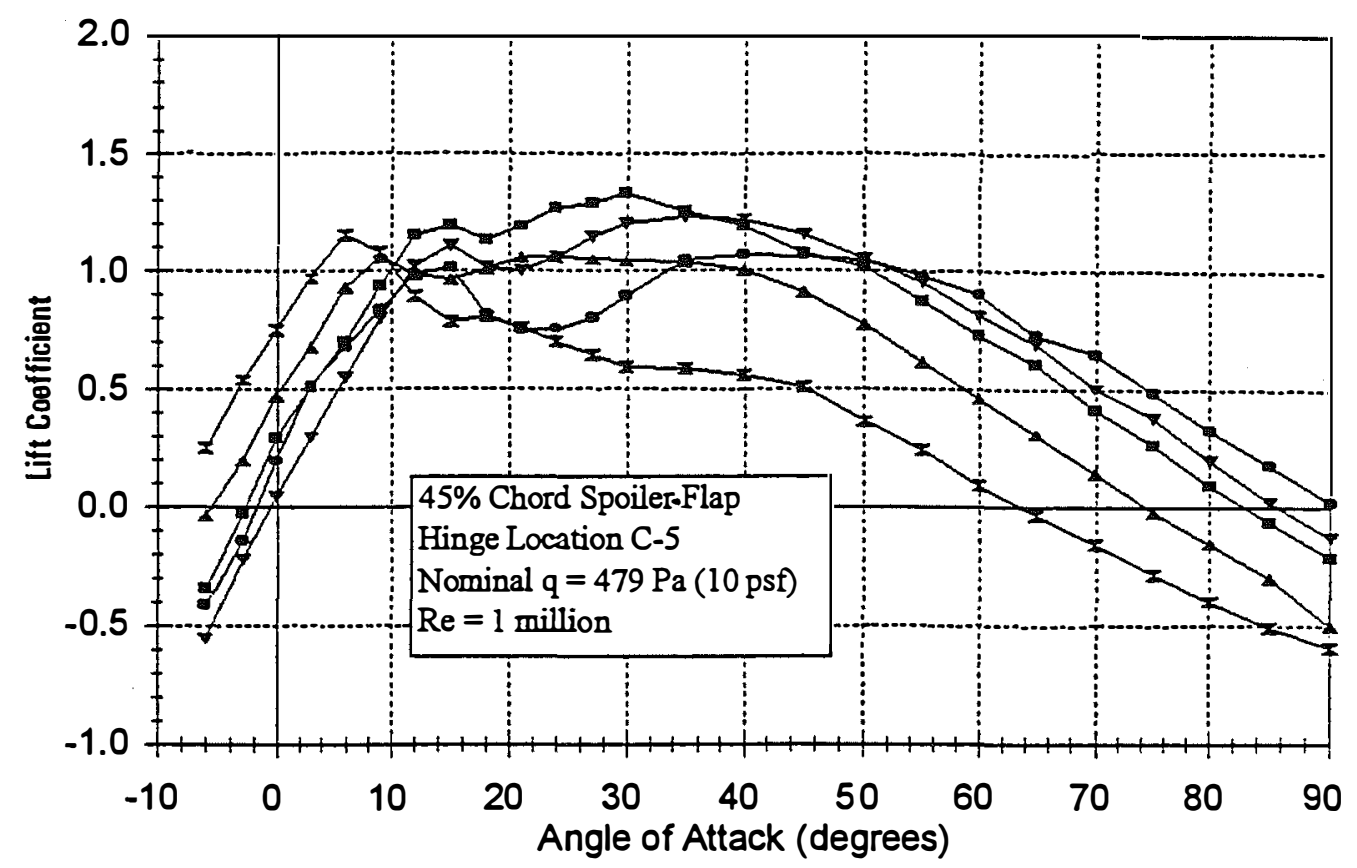

$\sim$ Delta $=0 \rightarrow$ Delta $=15 \rightarrow$ Delta $=30 \rightarrow$ Delta $=45 \rightarrow$ Delta $=60$

Figure A-15a. Lift Data, $\delta$-Sweep for $45 \%$ Chord Spoiler-Flap, Hinge C-5

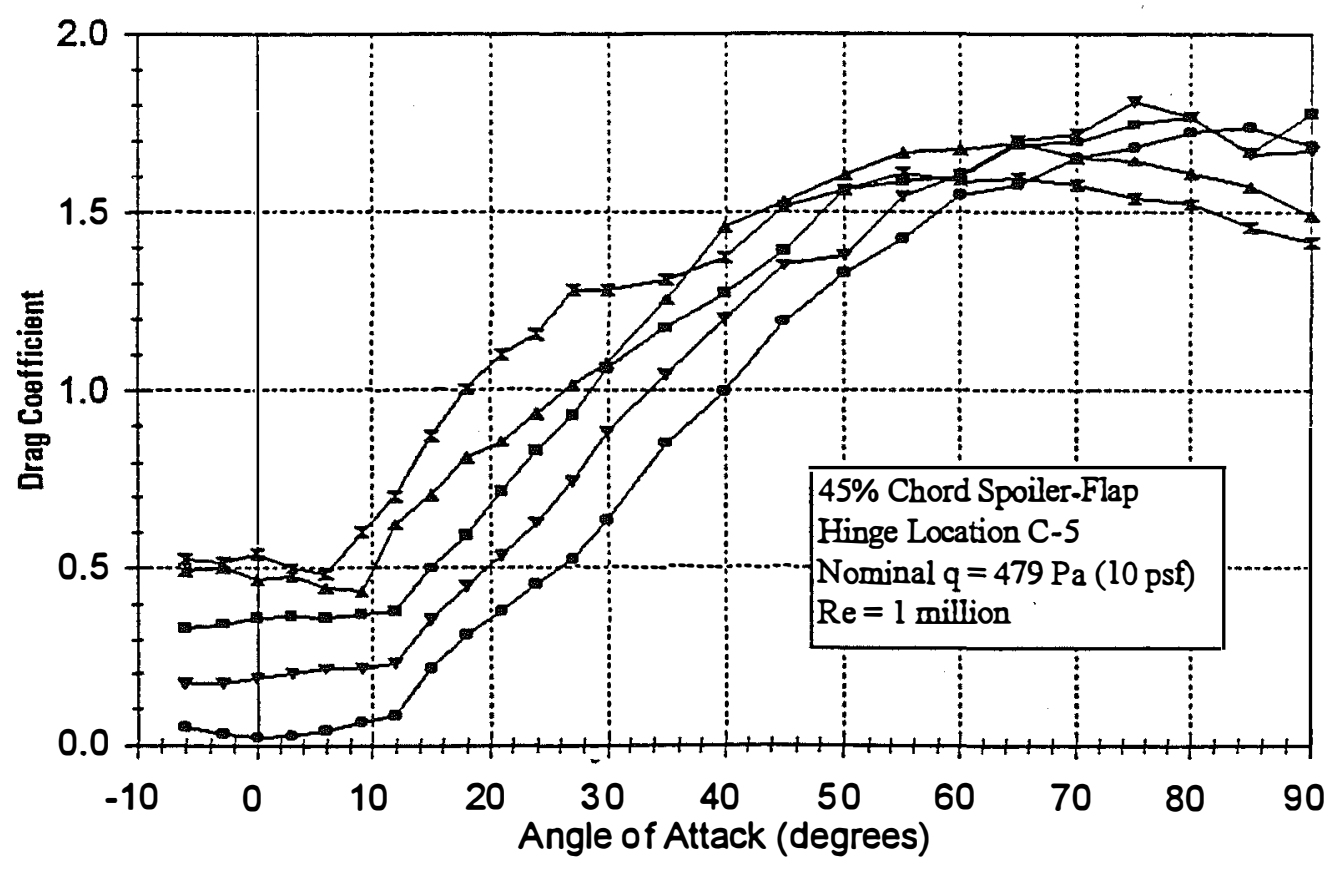

Delta $=0 \rightarrow$ Delta $=15 \rightarrow$ Delta $=30 \rightarrow$ Delta $=45 \rightarrow$ Delta $=60$

Figure A-15b. Drag Data, $\delta$-Sweep for $45 \%$ Chord Spoiler-Flap, Hinge C-5 


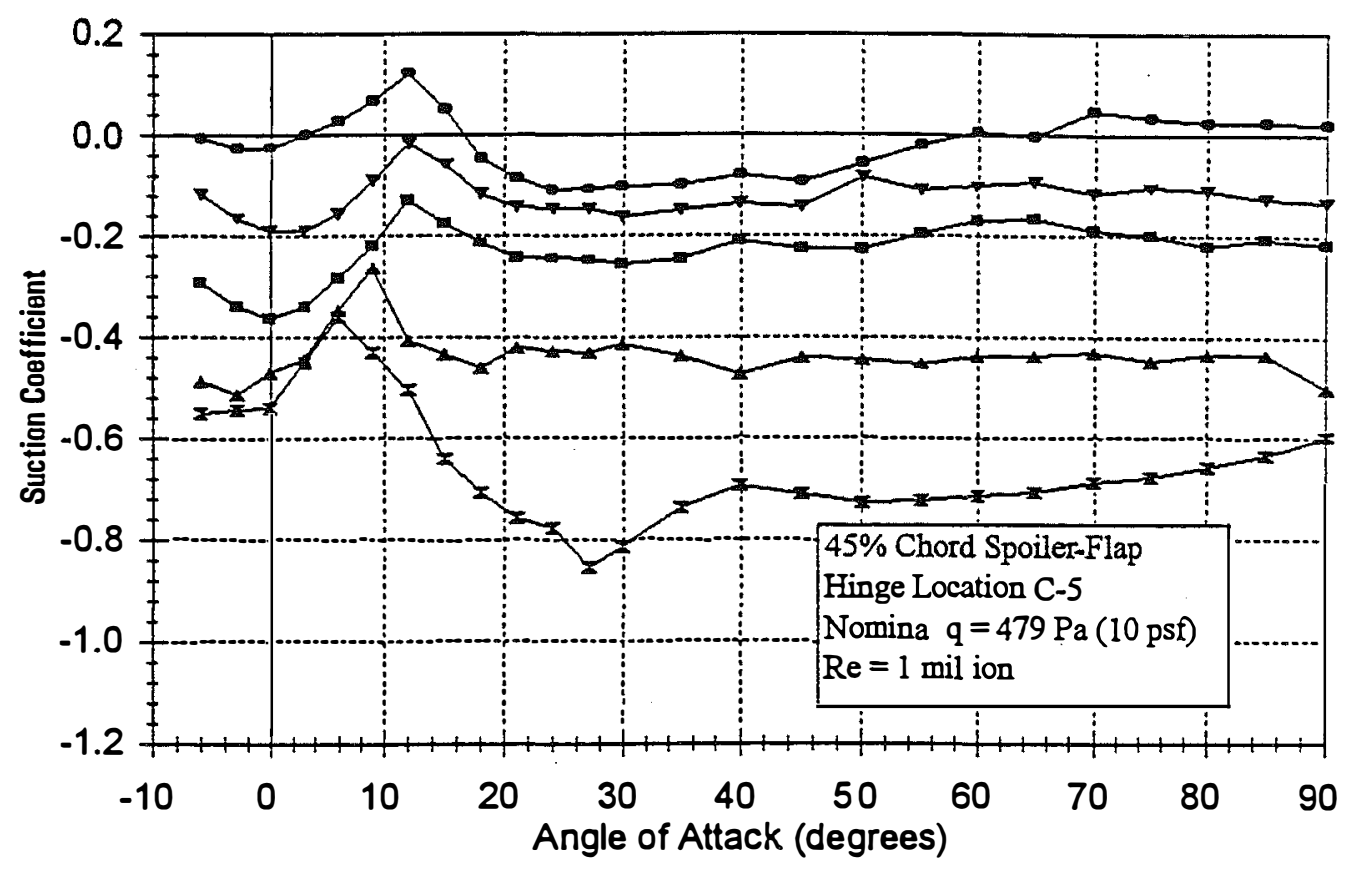

$\longrightarrow$ Delta $=0 \rightarrow$ Delta $=15 \rightarrow$ Delta $=30 \rightarrow$ Delta $=45 \rightarrow$ Delta $=60$

Figure A-15c. Suction Data, $\delta$-Sweep for $\mathbf{4 5 \%}$ Chord Spoiler.Flap, Hinge C-5

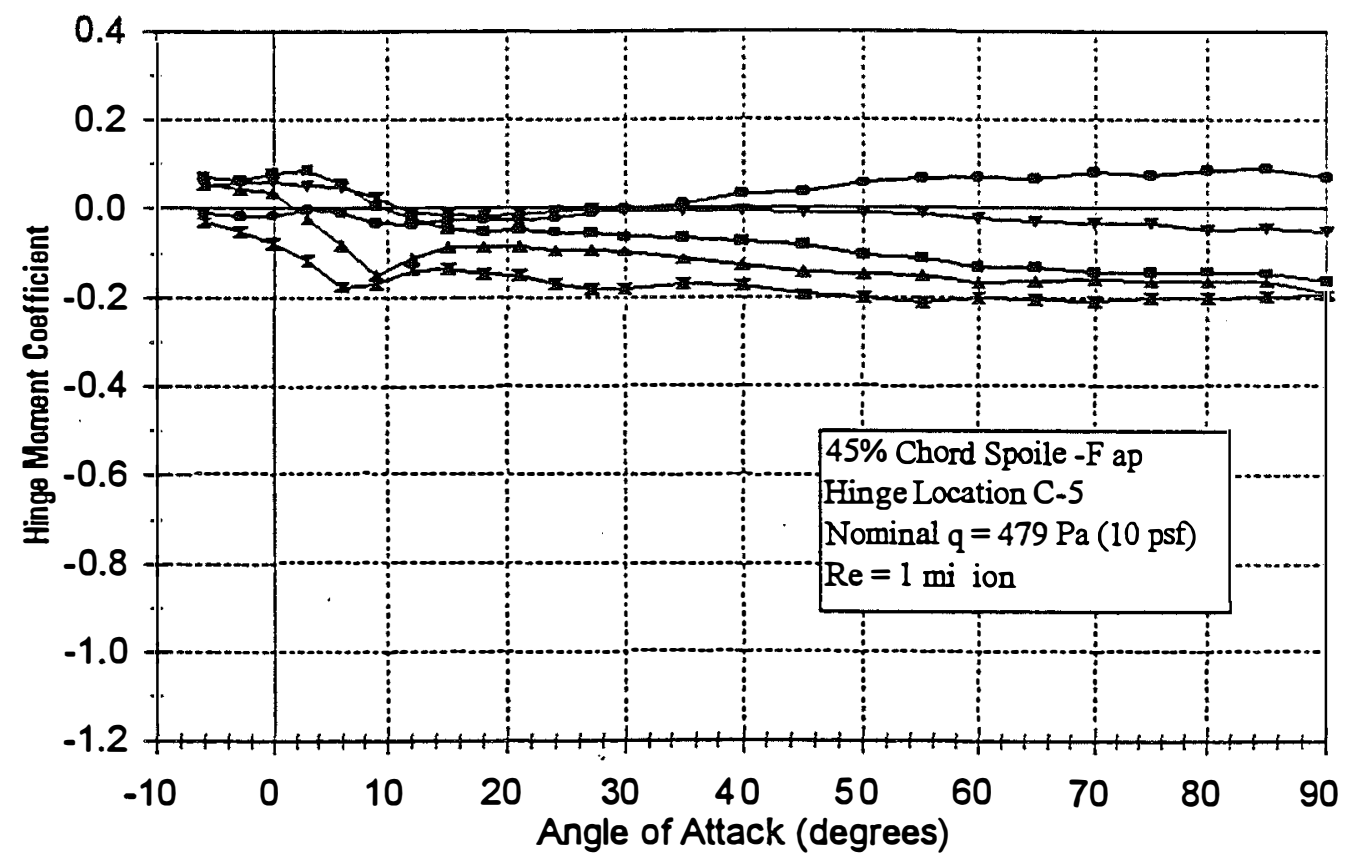

Delta $=0 \rightarrow$ Delta $=15 \rightarrow$ Delta $=30 \multimap$ Delta $=45 \rightarrow$ Delta $=60$

Figure A-15d. Hinge Moment Data, $\delta$-Sweep for $\mathbf{4 5 \%}$ Chord Spoiler-Flap, Hinge C-5 


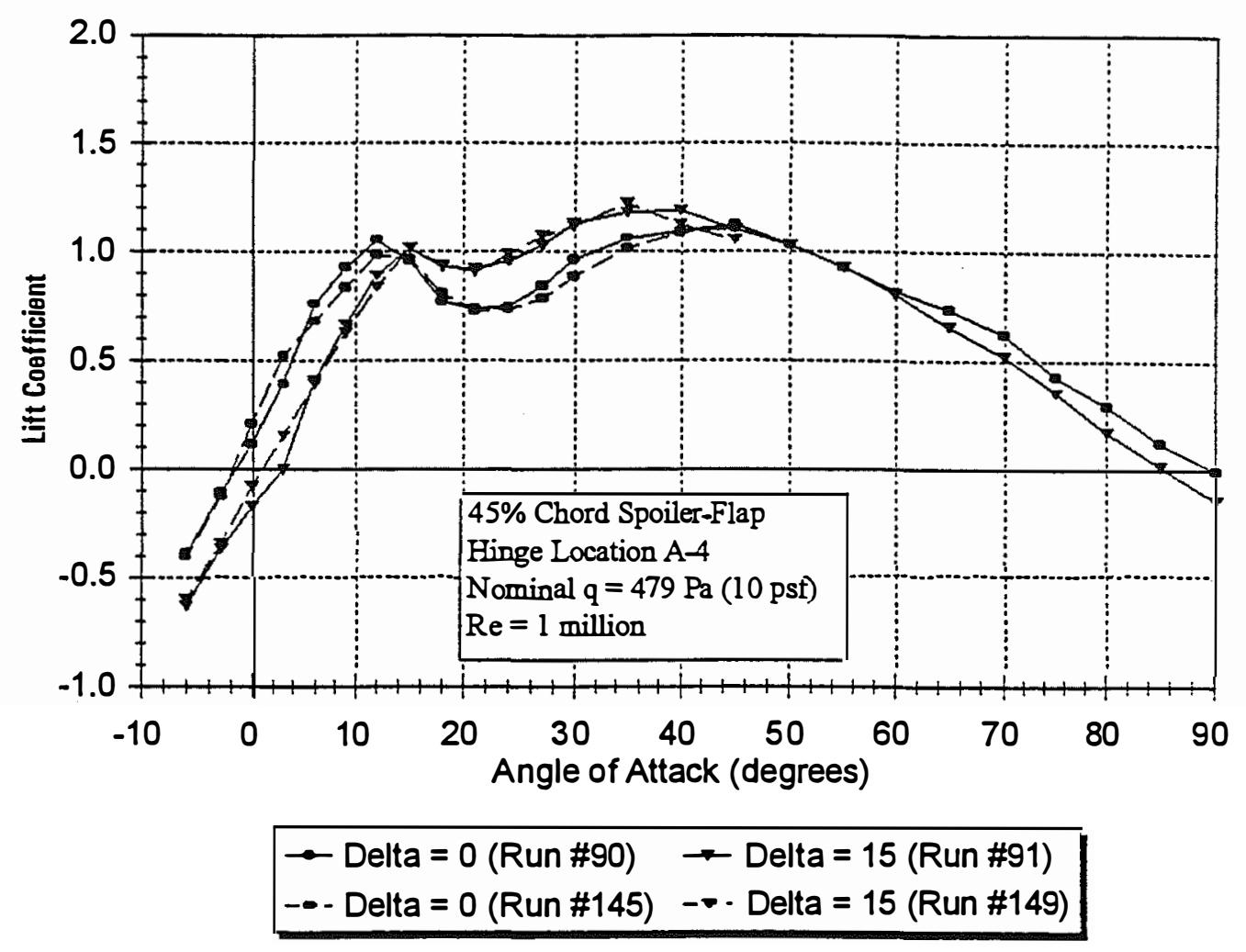

Figure A-16a. Lift Data, Repeatability Check on Spoiler-Flap

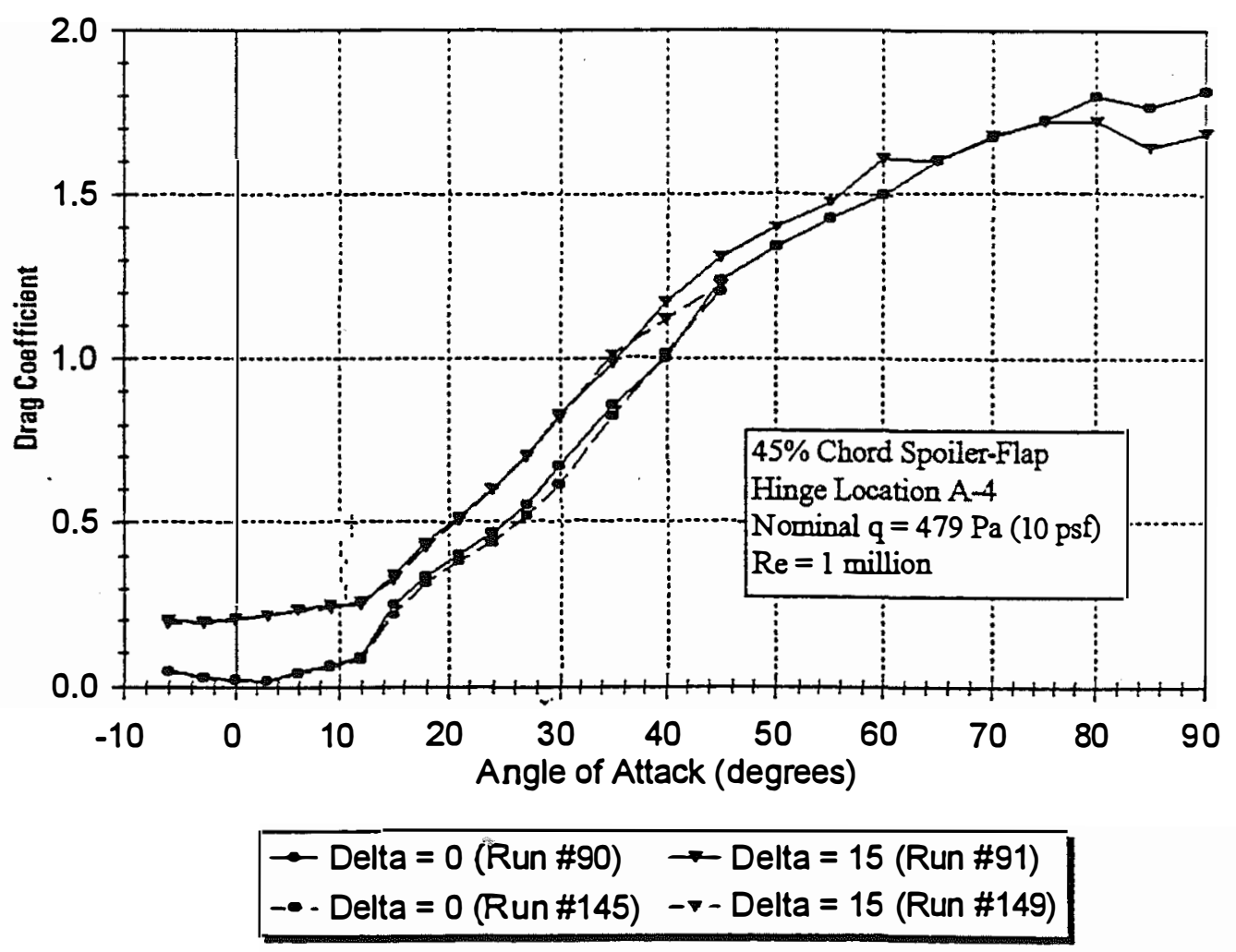

Figure A-16b. Drag Data, Repeatability Check on Spoiler-Flap 


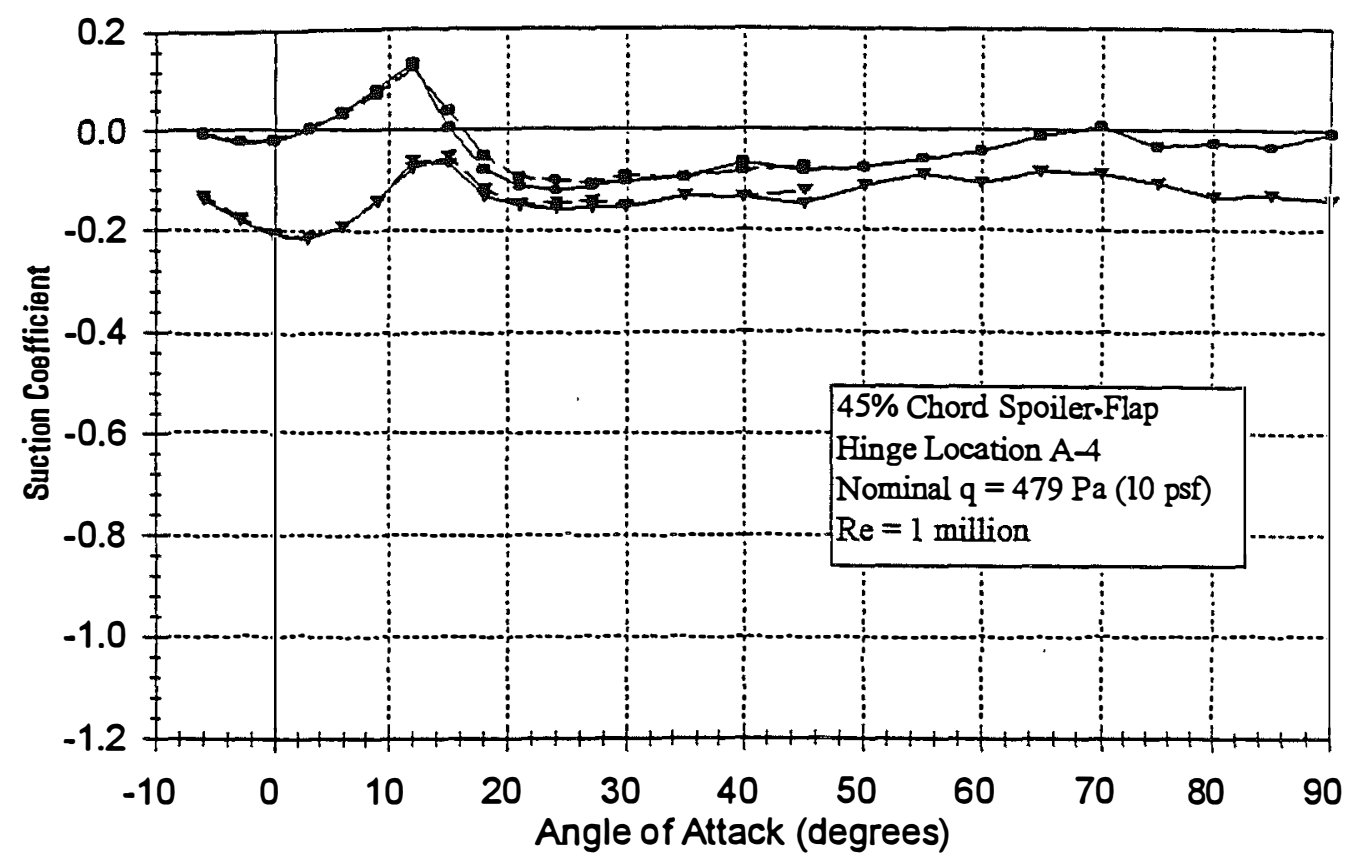

$$
\begin{aligned}
& \rightarrow \text { Delta = } 0 \text { (Run \#90) } \rightarrow \text { Delta = } 15 \text { (Run \#91) } \\
& \text {-- Delta = } 0 \text { (Run \#145) }
\end{aligned}
$$

Figure A-16c. Suction Data, Repeatability Check on Spoiler-Flap

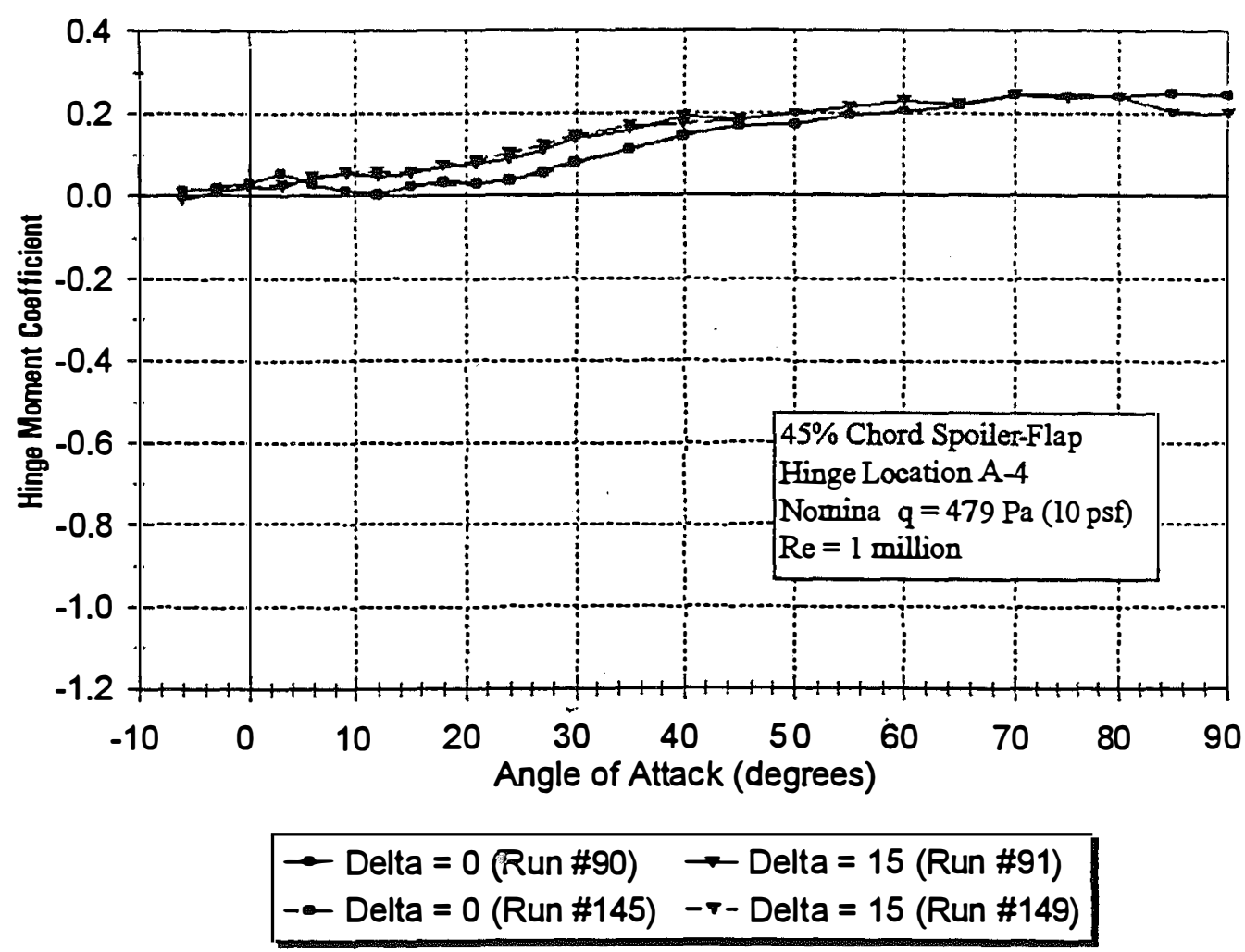

Figure A-16d. Hinge Moment Data, Repeatability Check on Spoiler.Flap 


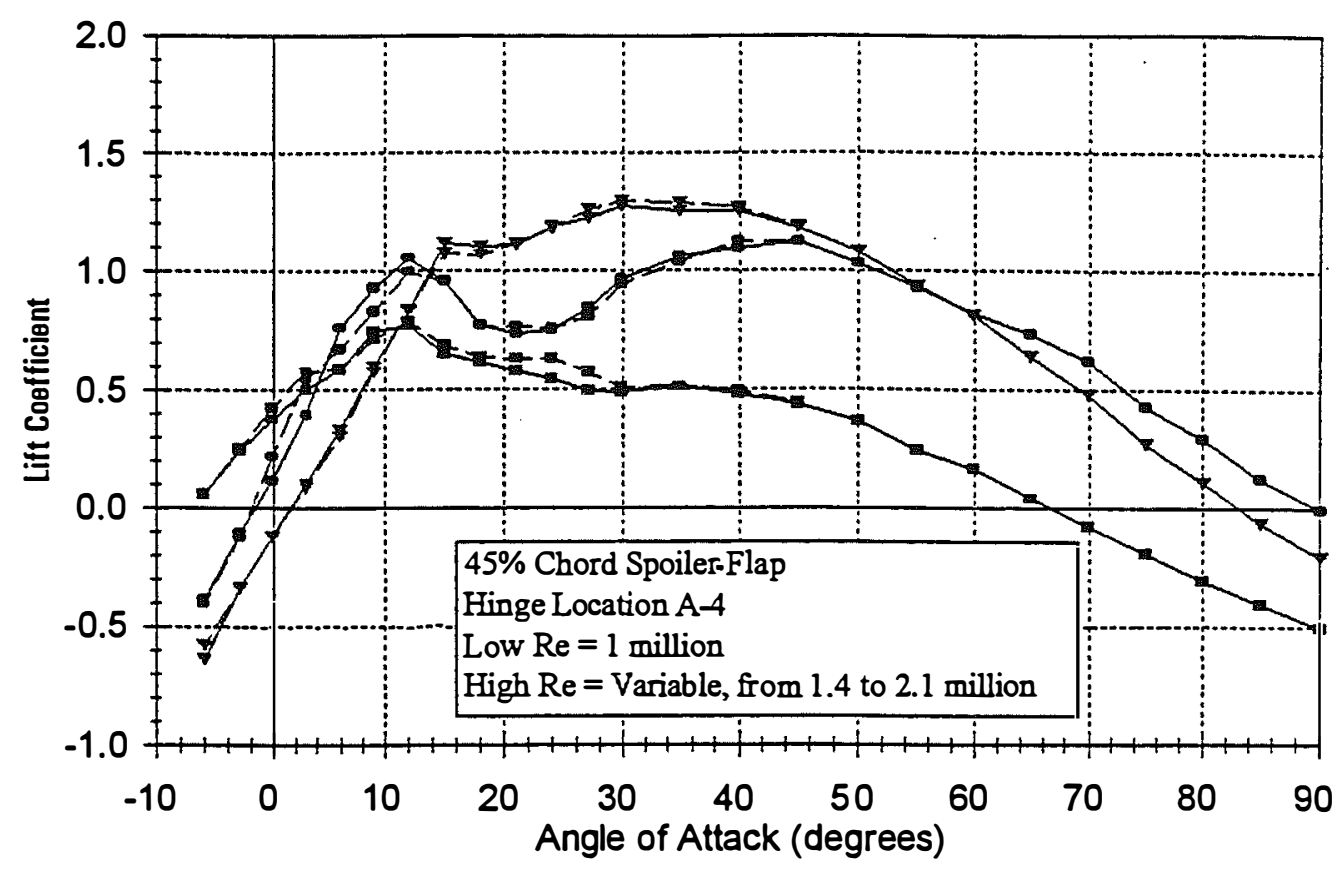

$\rightarrow$ Delta $=0($ Low Re $) \rightarrow-$ Delta $=0($ High Re $) \rightarrow$ Delta $=30($ Low Re $)$
-- Delta $=30($ High Re $) \rightarrow$ Delta $=60($ Low Re $) \rightarrow-$ Delta $=60($ High Re $)$

Figure A-17a. Lift Data, Reynolds Number Effect on Spoiler.Flap

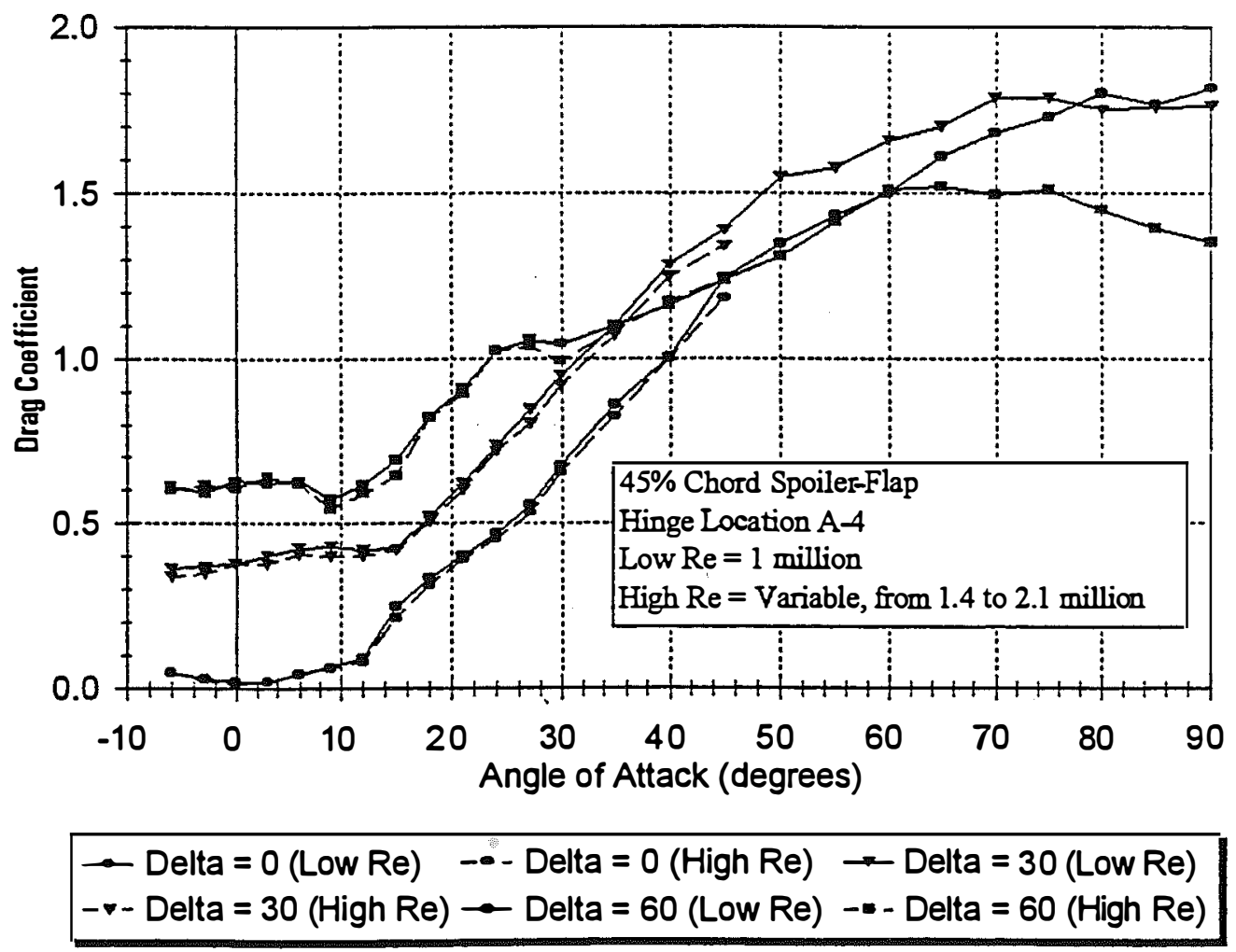

Figure A-17b. Drag Data, Reynolds Number Effect on Spoiler-Flap 


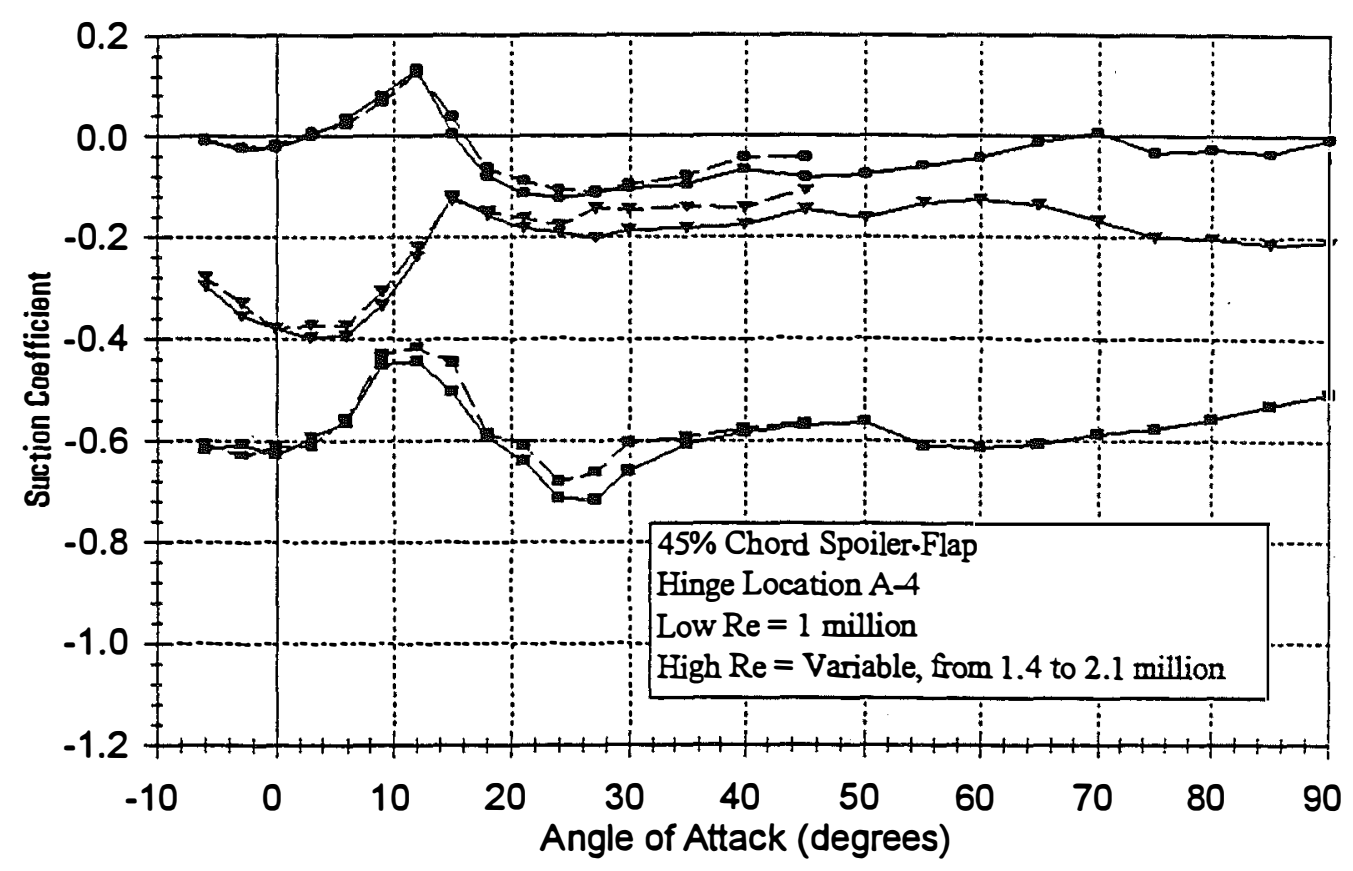

$$
\begin{aligned}
& \rightarrow-\text { Delta }=0(\text { Low Re }) \rightarrow-\text { Delta }=0(\text { High Re }) \rightarrow \text { Delta }=30(\text { Low Re }) \\
& \rightarrow-\text { Delta }=30(\text { High Re })-\text { Delta }=60(\text { Low Re }) \rightarrow-\text { Delta }=60(\text { High Re })
\end{aligned}
$$

Figure A-17c. Suction Data, Reynolds Number Effect on Spoiler-Flap

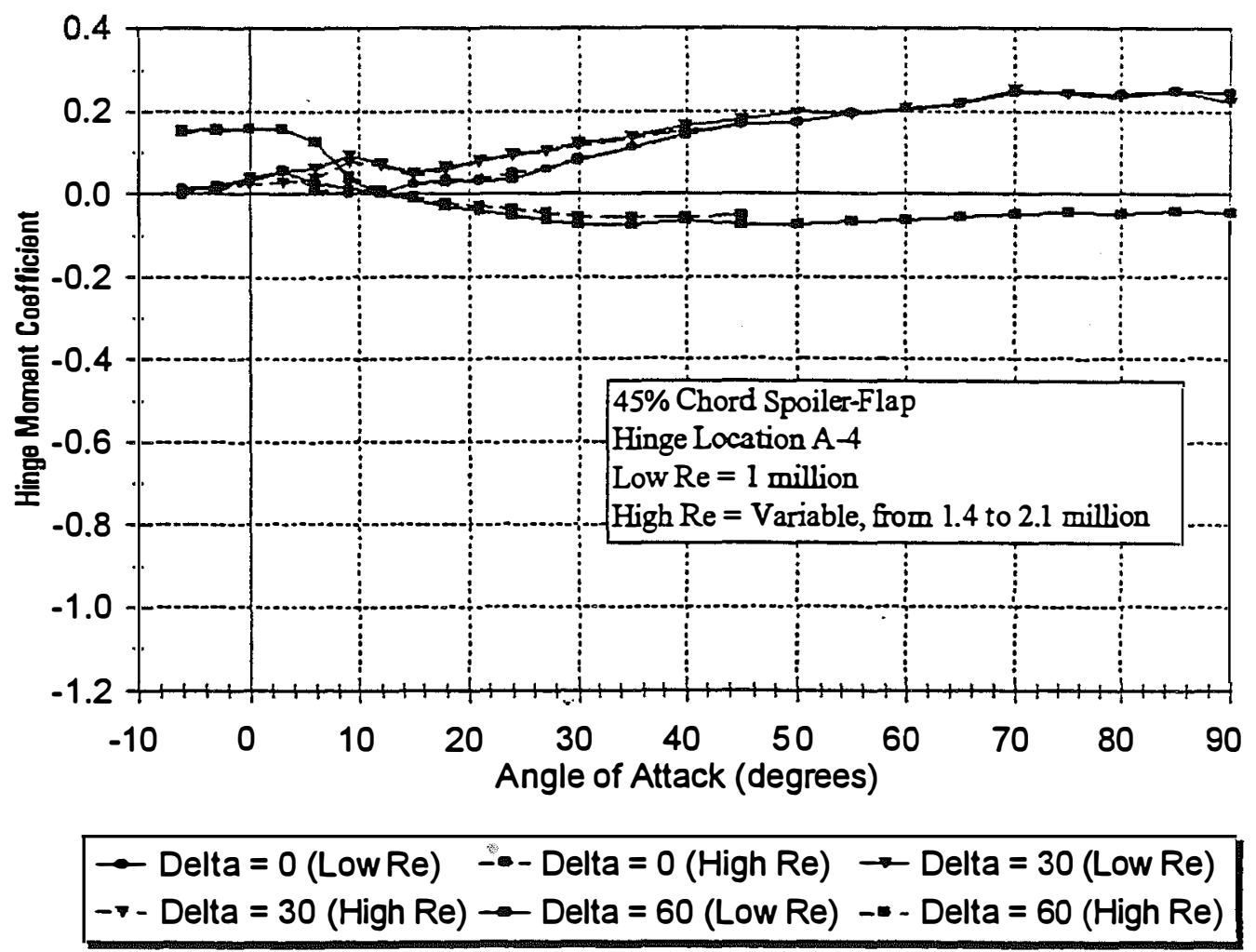

Figure A-17d. Hinge Moment Data, Reynolds Number Effect on Spoiler-Flap 


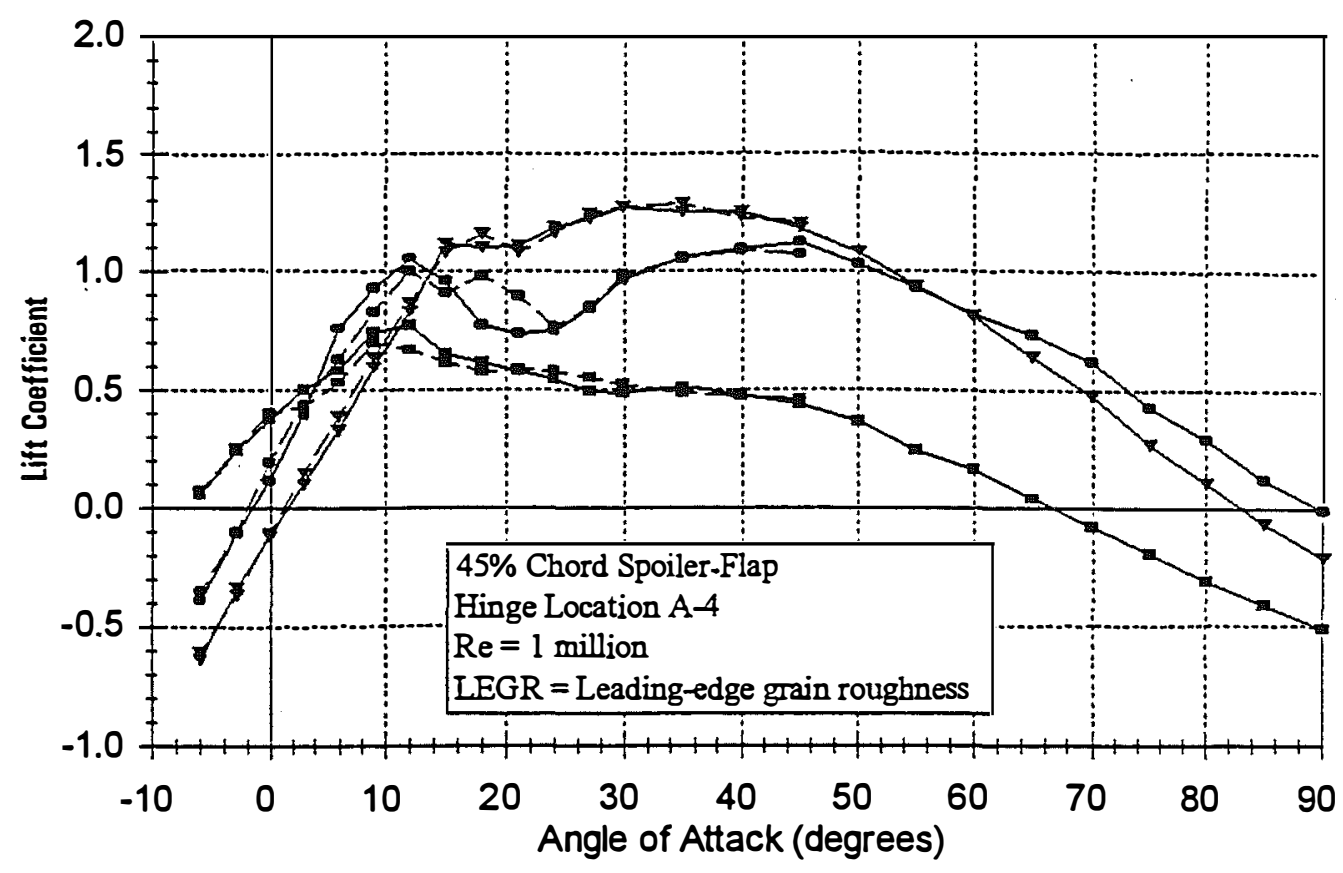

$$
\begin{aligned}
& \rightarrow \text { Delta }=0 \text { (Clean) } \rightarrow \text { Delta }=0(\text { LEGR) } \rightarrow \text { Delta }=30 \text { (Clean) } \\
& \rightarrow-\text { Delta }=30(\text { LEGR) } \rightarrow \text { Delta }=60(\text { Clean }) \rightarrow-\text { Delta }=60 \text { (LEGR) }
\end{aligned}
$$

Figure A-18a. Lift Data, Effect of Leading-Edge Roughness on Spoiler.Flap

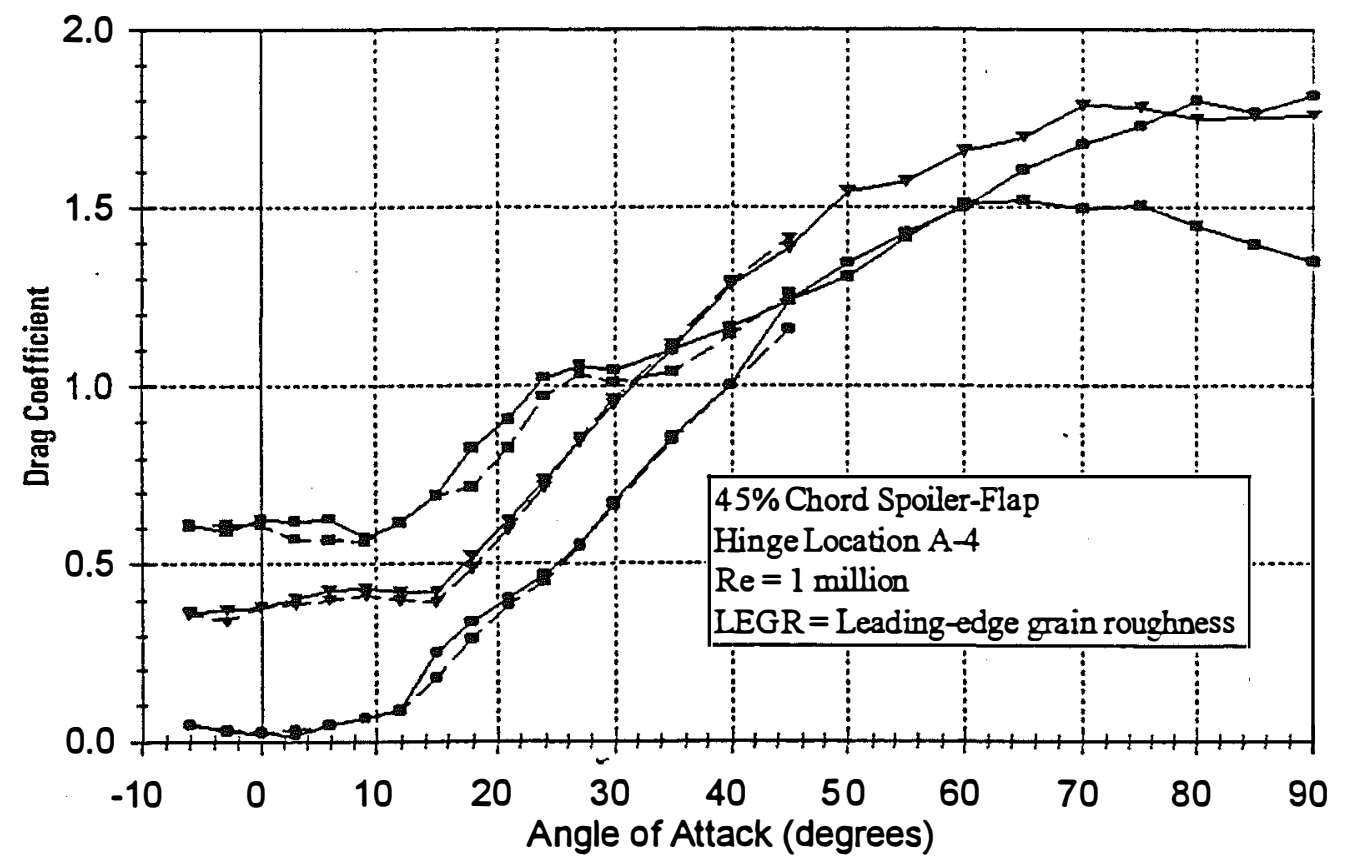

$$
\begin{aligned}
& \rightarrow \text { Delta }=0 \text { (Clean) } \rightarrow-\text { Delta }=0 \text { (LEGR) } \rightarrow \text { Delta }=30 \text { (Clean) } \\
& \rightarrow-\text { Delta }=30(\text { LEGR) } \rightarrow \text { Delta }=60 \text { (Clean) } \rightarrow-\text { Delta }=60 \text { (LEGR) }
\end{aligned}
$$

Figure A-18b. Drag Data, Effect of Leading-Edge Roughness on Spoiler-Flap 


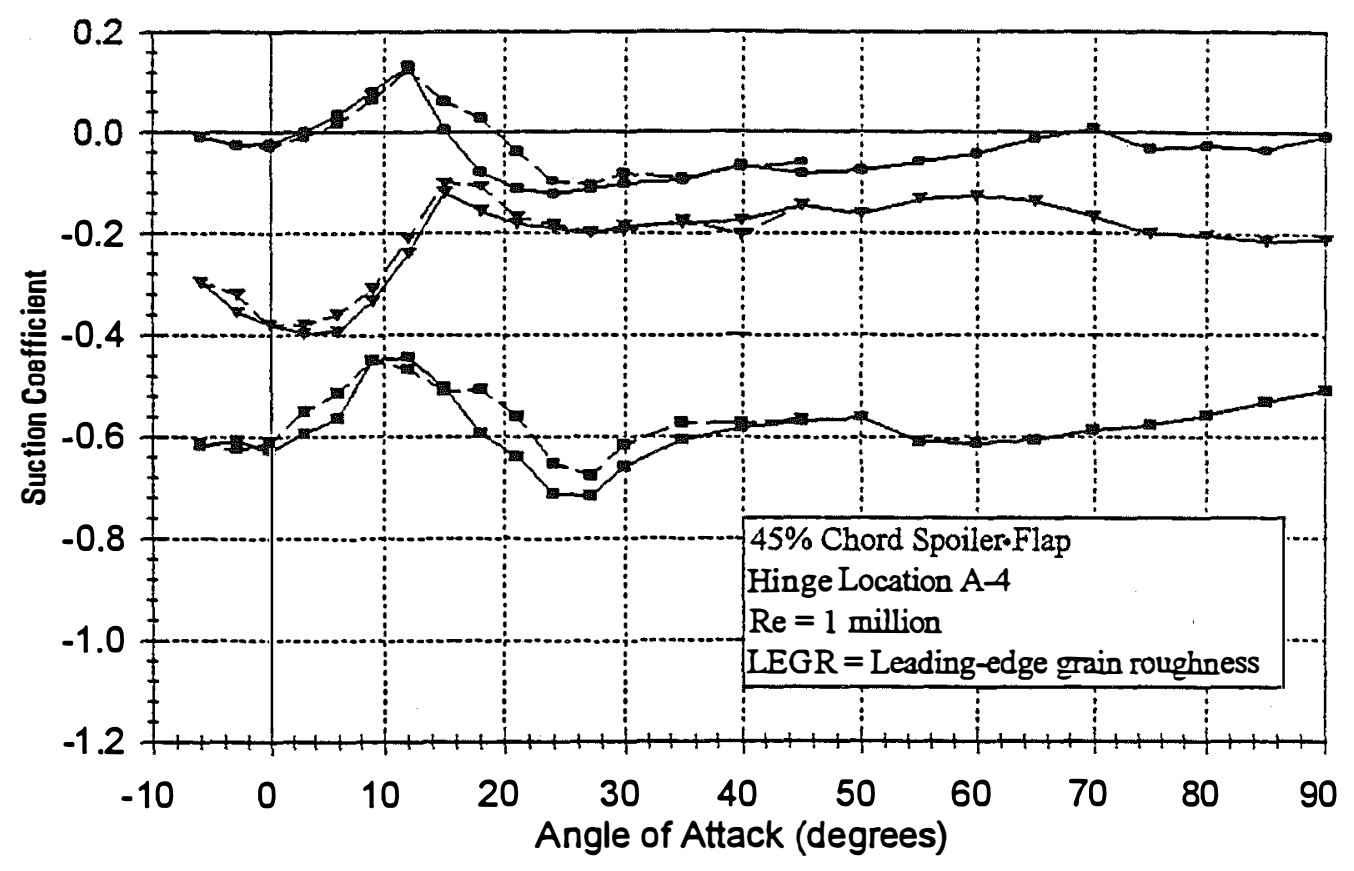

$$
\begin{aligned}
& \rightarrow \text { Delta }=0 \text { (Clean) } \rightarrow-\text { Delta }=0 \text { (LEGR) } \rightarrow \text { Delta }=30 \text { (Clean) } \\
& \rightarrow-\text { Delta }=30(\text { LEGR }) \rightarrow \text { Delta }=60 \text { (Clean) } \rightarrow-\text { Delta }=60(\text { LEGR) }
\end{aligned}
$$

Figure A-18c. Suction Data, Effect of Leading-Edge Roughness on Spoiler-Flap

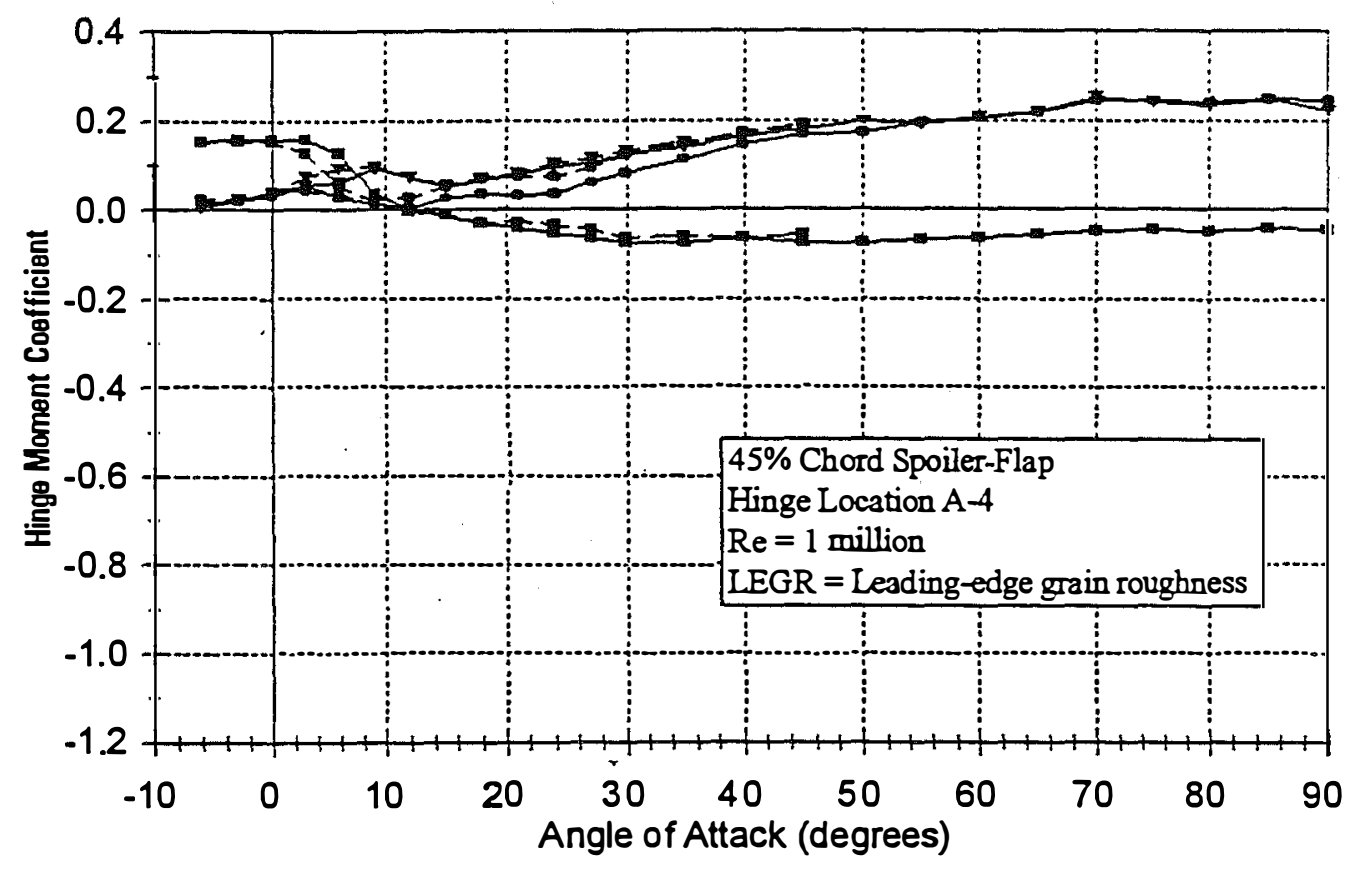

$$
\begin{aligned}
& \rightarrow \text { Delta }=0 \text { (Clean) } \rightarrow-\text { Delta }=0 \text { (LEGR) } \rightarrow \text { Delta }=30(\text { Clean }) \\
& \rightarrow-\text { Delta }=30(\text { LEGR }) \rightarrow \text { Delta }=60(\text { Clean }) \rightarrow-\text { Delta }=60(\text { LEGR })
\end{aligned}
$$

Figure A-18d. Hinge Moment Data, Effect of Leading-Edge Roughness on Spoiler-Flap 


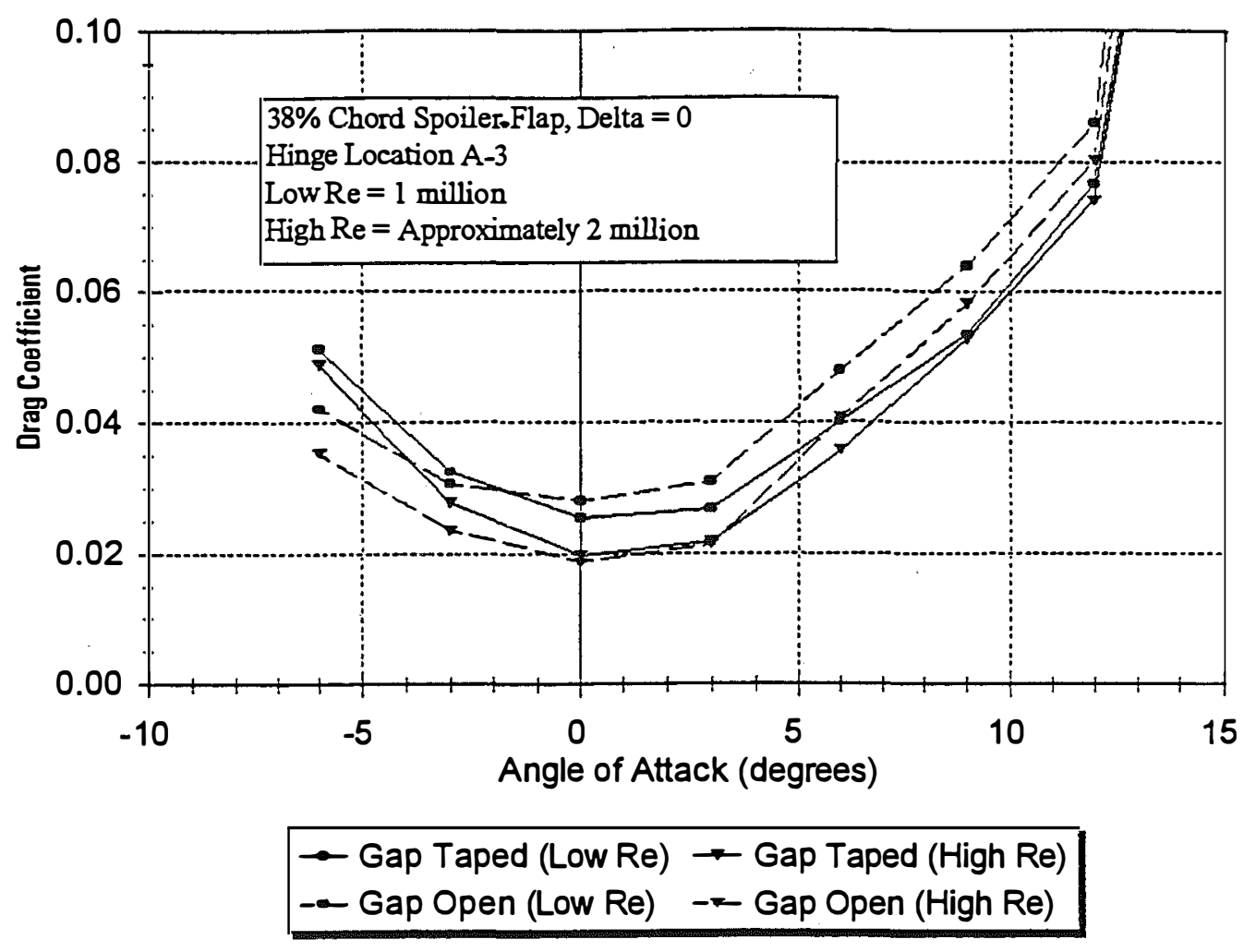

Figure A-19. Effect of Gap Seal on 38\% Chord Device

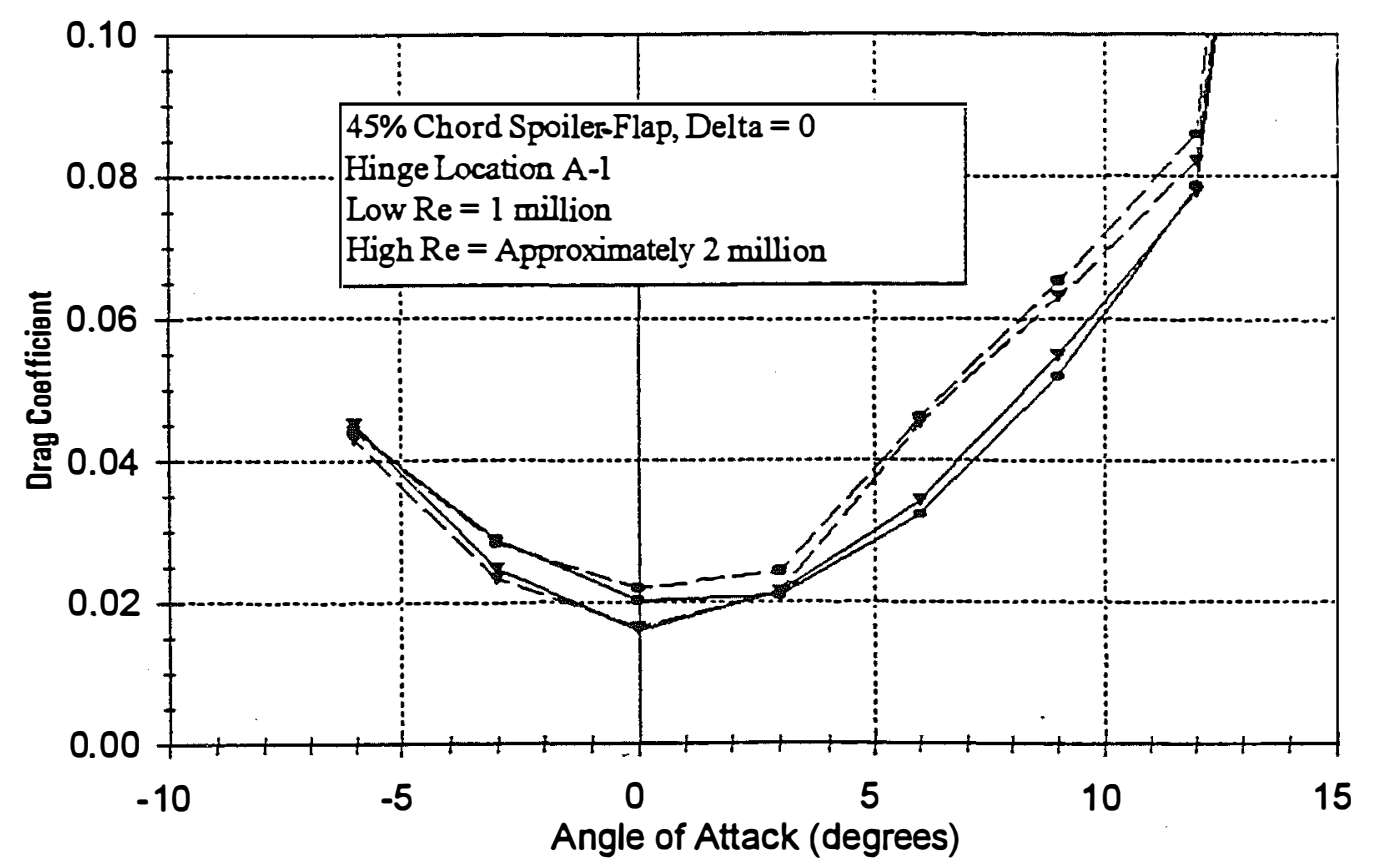

$$
\begin{aligned}
& \rightarrow \text { - Gap Taped (Low Re) } \rightarrow \text { - Gap Taped (High Re) } \\
& \rightarrow-\text { Gap Open (Low Re) } \rightarrow \text { - Gap Open (High Re) }
\end{aligned}
$$

Figure A-20. Effect of Gap Seal on $45 \%$ Chord Device 


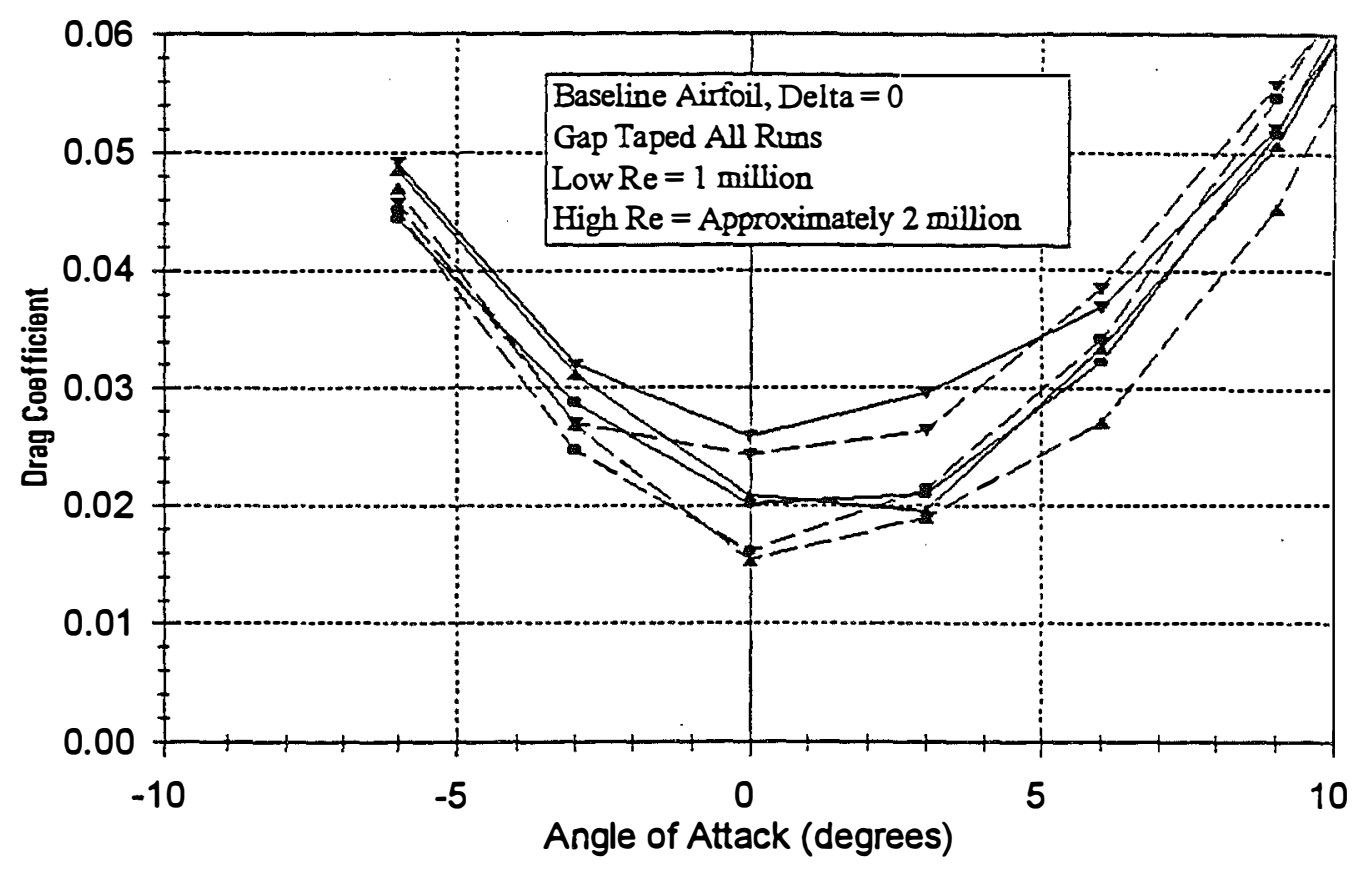

$\rightarrow$ Clean (Low Re) $\quad-$ - Clean (High Re) $\rightarrow 15$ mil Tape (Low Re)

--15 mil Tape (High Re) $\rightarrow 6$ mil Tape (Low Re) $-₫-6$ mil Tape (High Re)

Figure A-21. Effect of Leading-Edge Tape on Clean $\mathbf{8 8 1 0}$ 


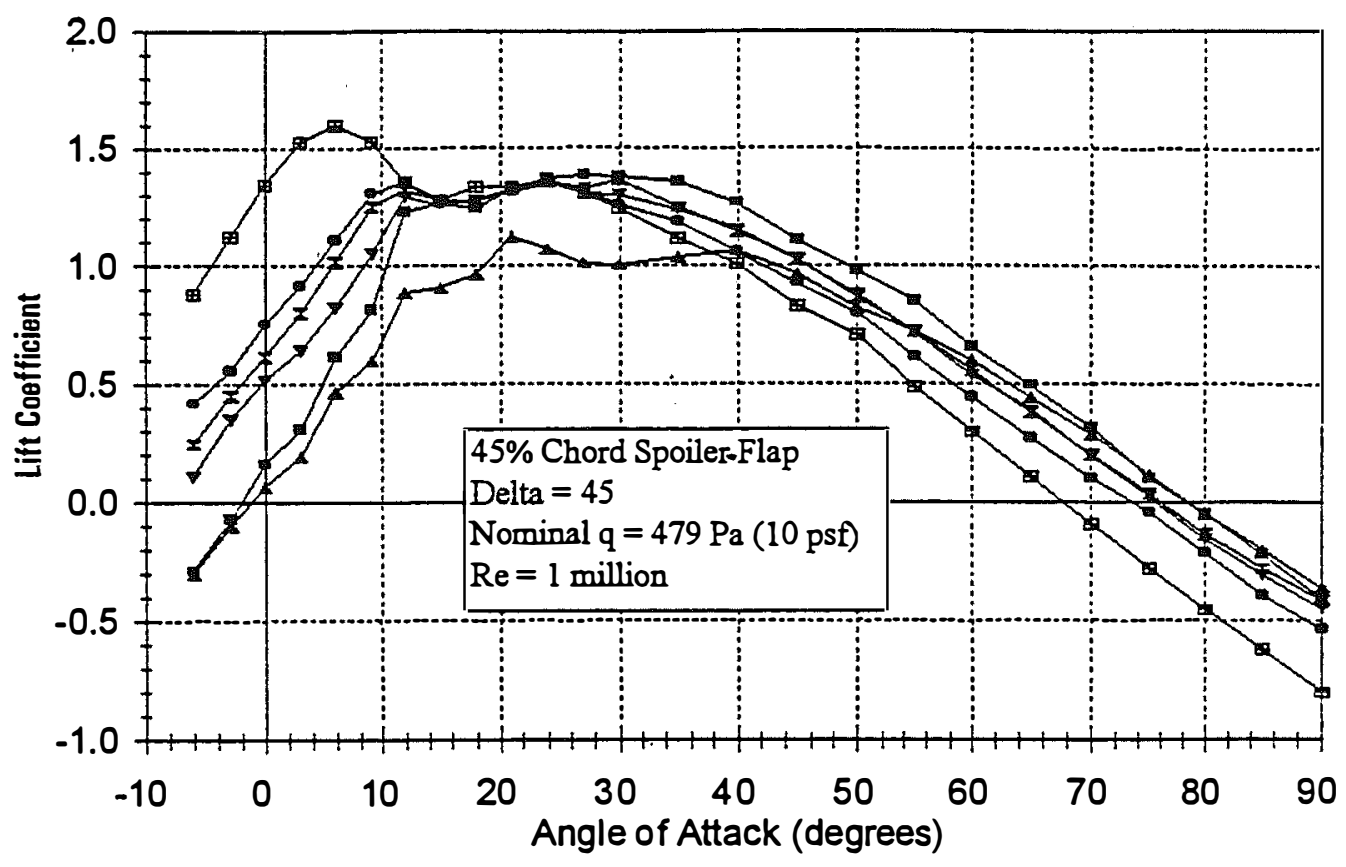

$\rightarrow$ Hinge A-1 $\rightarrow$ Hinge A-2 $\rightarrow$ Hinge A-3

Hinge $A-4 \rightarrow-$ Hinge $B-3 \rightarrow$ Hinge $C-1$

Figure A-22a. Lift Data, Effect of Hinge Location on Device at $\delta=45^{\circ}$

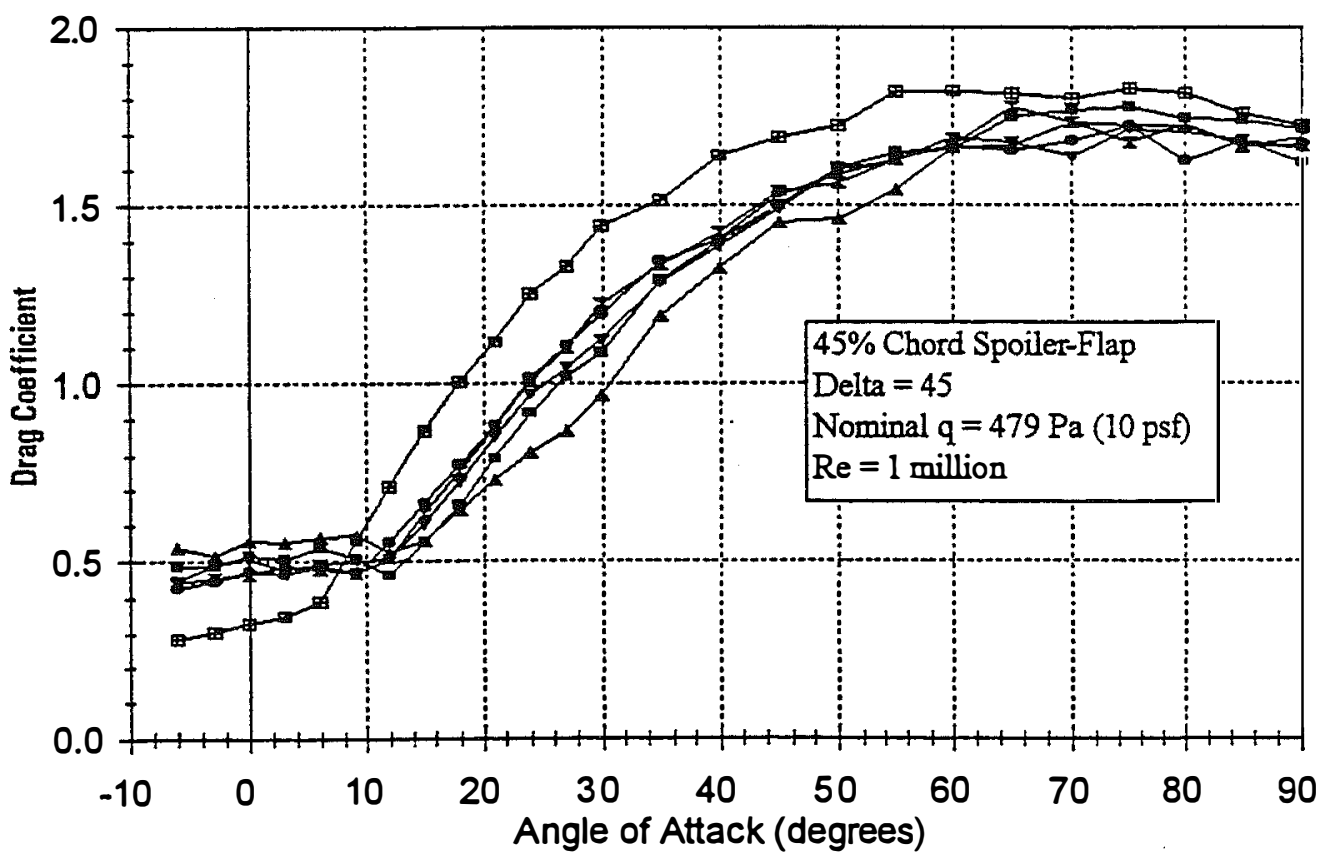

$\rightarrow-$ Hinge A-1 $\rightarrow$ Hinge A-2 $\rightarrow$ Hinge A-3
$\rightarrow$ Hinge $A-4 \rightarrow$ Hinge $B-3 \rightarrow-$ Hinge C-1

Figure A-22b. Drag Data, Effect of Hinge Location on Device at $\delta=45^{\circ}$ 


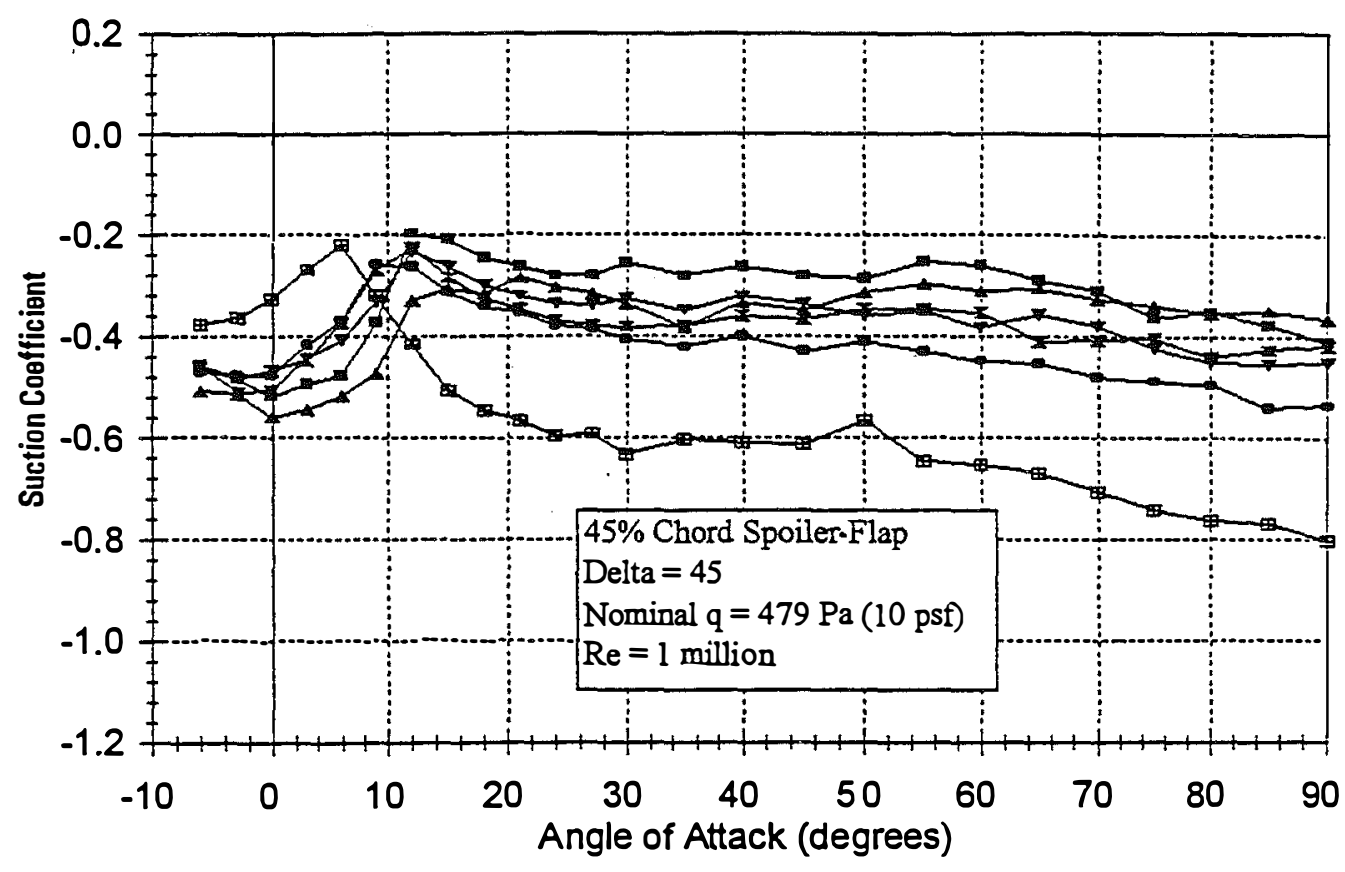

$\rightarrow$ Hinge $A-1 \rightarrow$ Hinge $A-2 \rightarrow$ Hinge $A-3$
- Hinge $A-4 \rightarrow$ Hinge $B-3 \rightarrow$ Hinge $C-1$

Figure A-22c. Suction Data, Effect of Hinge Location on Device at $\delta=45^{\circ}$

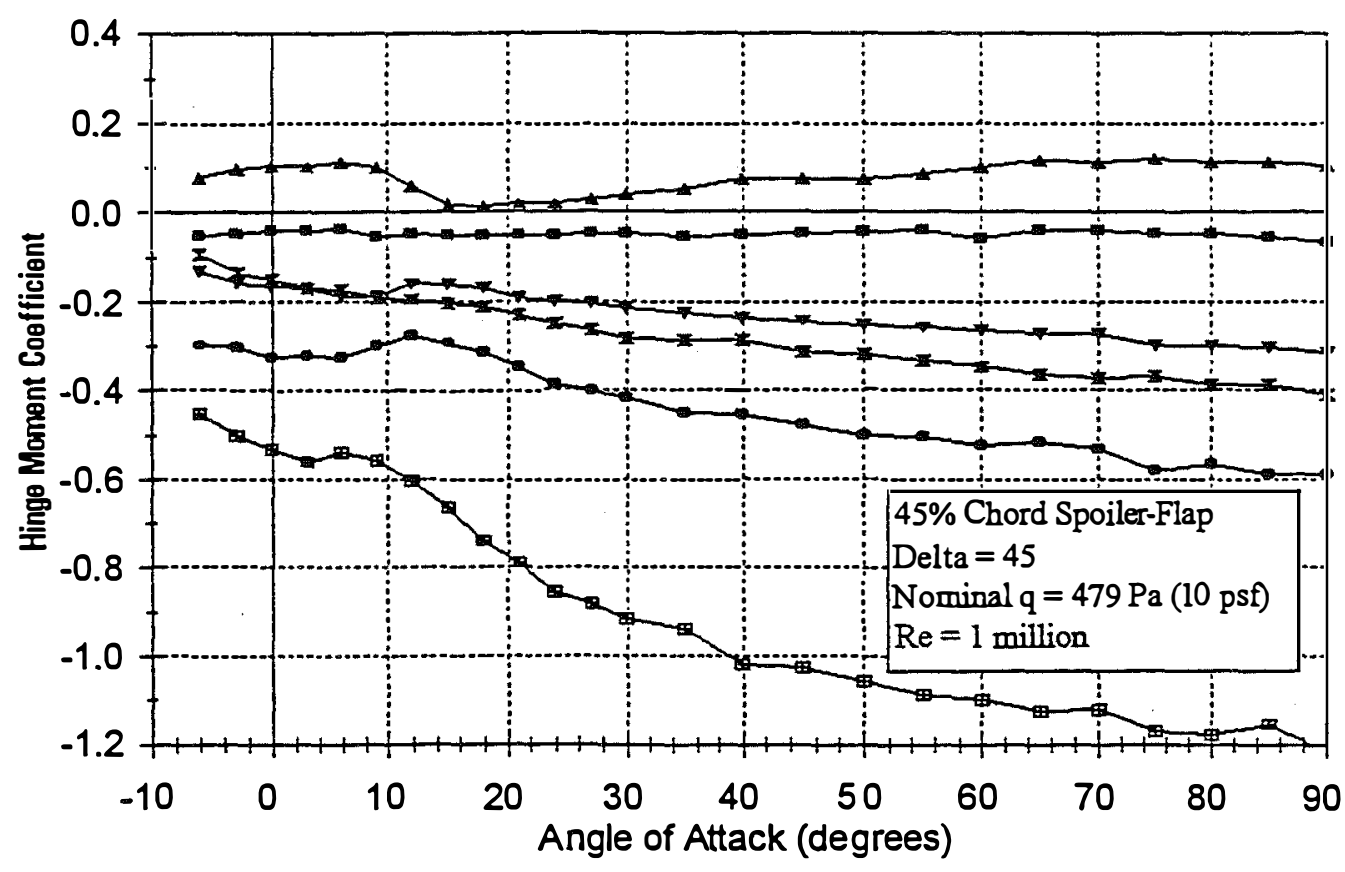

$\rightarrow$ Hinge $A-1 \rightarrow$ Hinge $A-2 \rightarrow$ Hinge $A-3$

$\rightarrow$ Hinge $A-4 \rightarrow$ Hinge $B-3 \rightarrow$ Hinge $C-1$

Figure A-22d. Hinge Moment Data, Effect of Hinge Location on Device at $\delta=45^{\circ}$ 

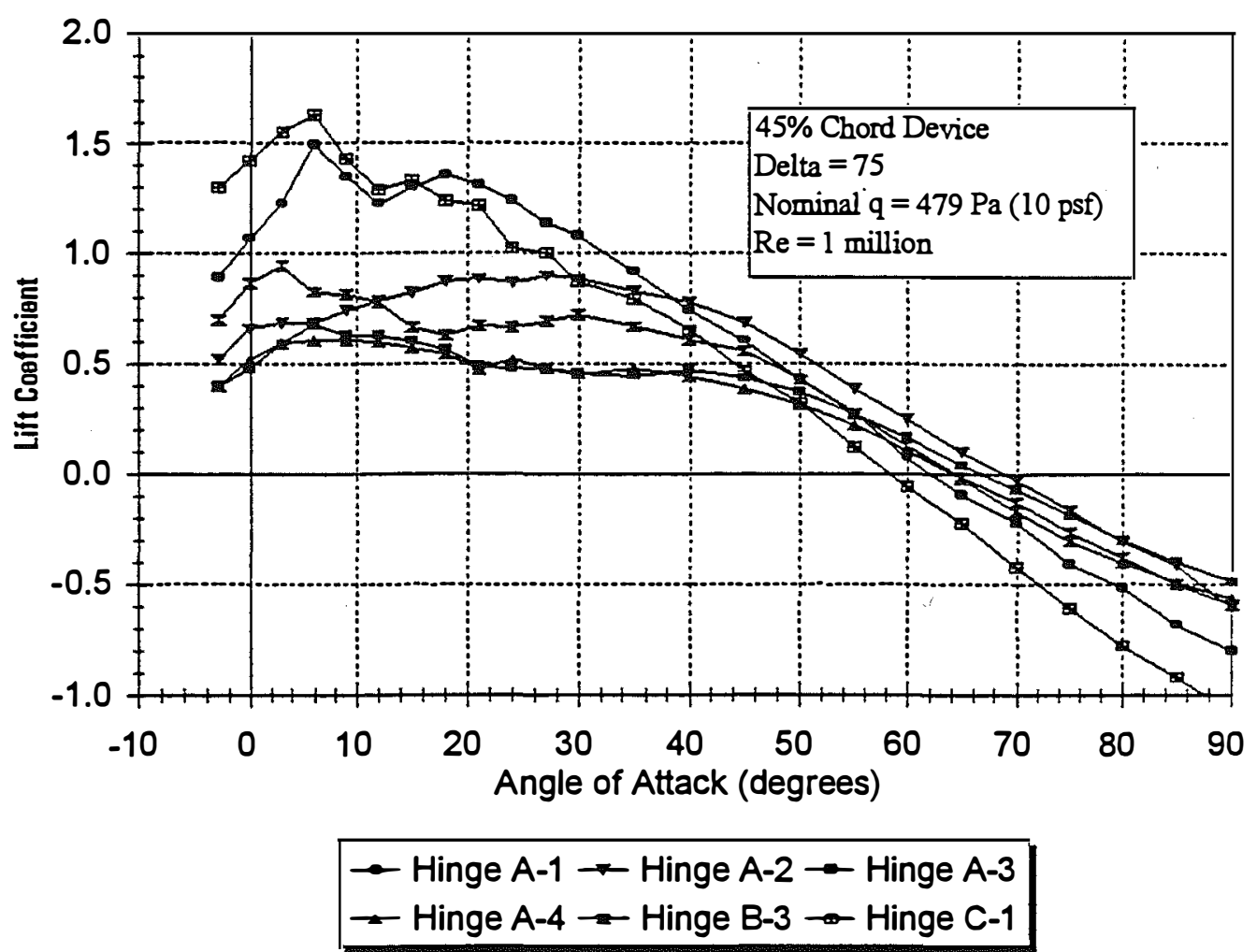

Figure A-23a. Lift Data, Effect of Hinge Location on Device at $\delta=75^{\circ}$

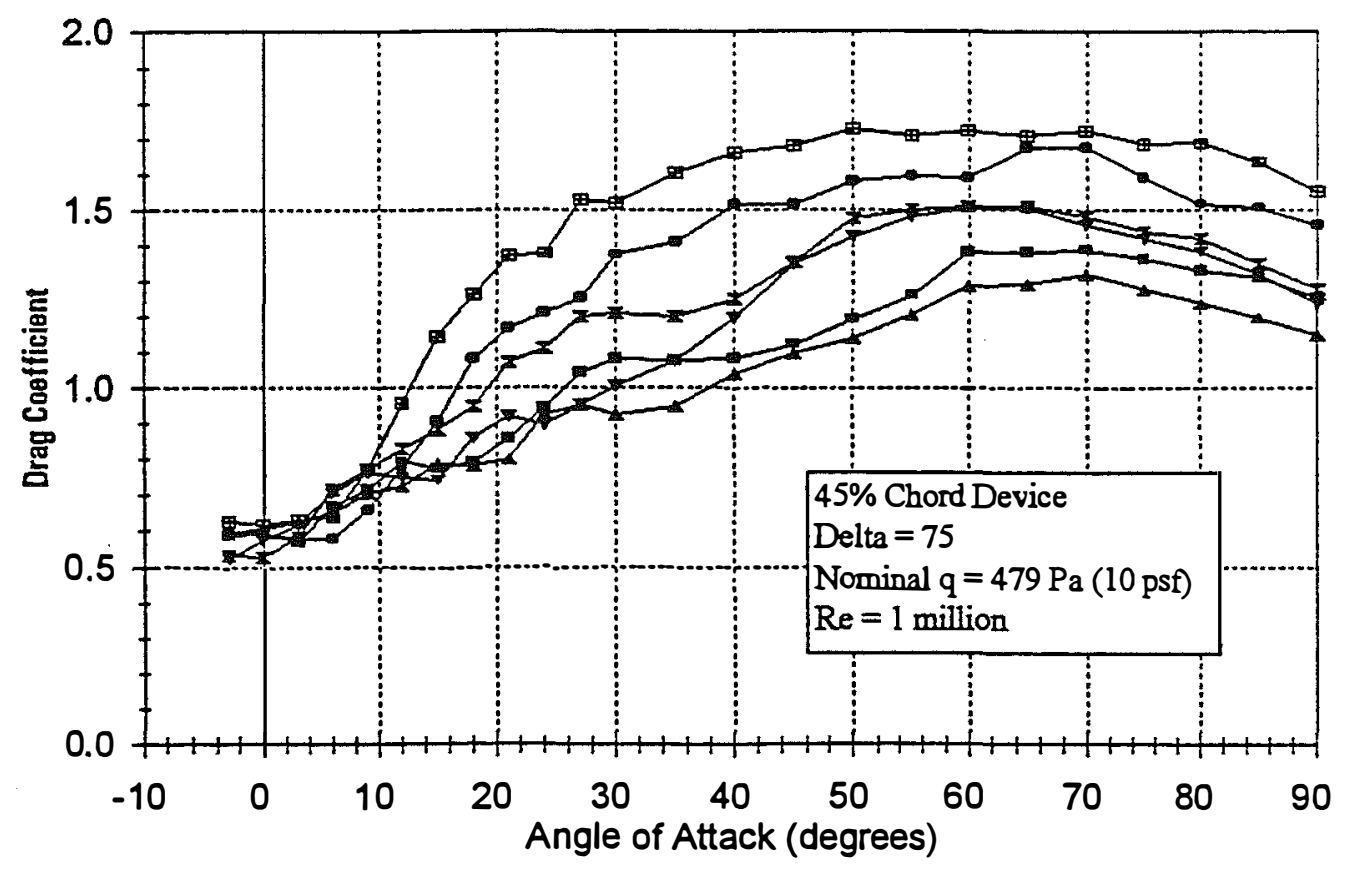

$\rightarrow$ Hinge A-1 $\rightarrow$ Hinge A-2 $\rightarrow$ Hinge A-3
- Hinge A-4 $\rightarrow-$ Hinge B-3 $\rightarrow$ Hinge C-1

Figure A-23b. Drag Data, Effect of Hinge Location on Device at $\delta=75^{\circ}$ 


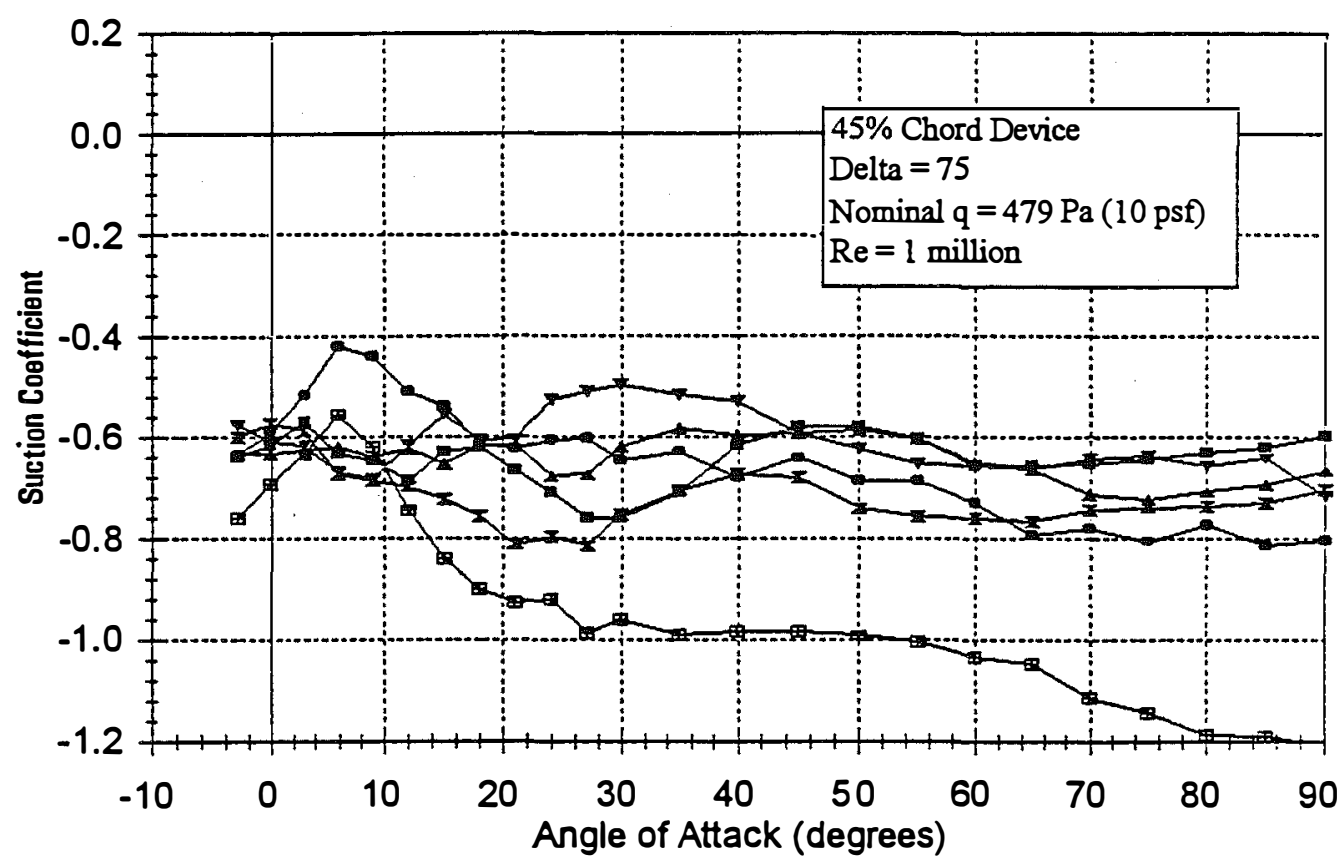

$\rightarrow$ Hinge A-1 $\rightarrow$ Hinge A-2 $\rightarrow$ Hinge A-3
- Hinge A-4 $\rightarrow-$ Hinge B-3 $\rightarrow$ Hinge C-1

Figure A-23c. Suction Data, Effect of Hinge Location on Device at $\delta=75^{\circ}$

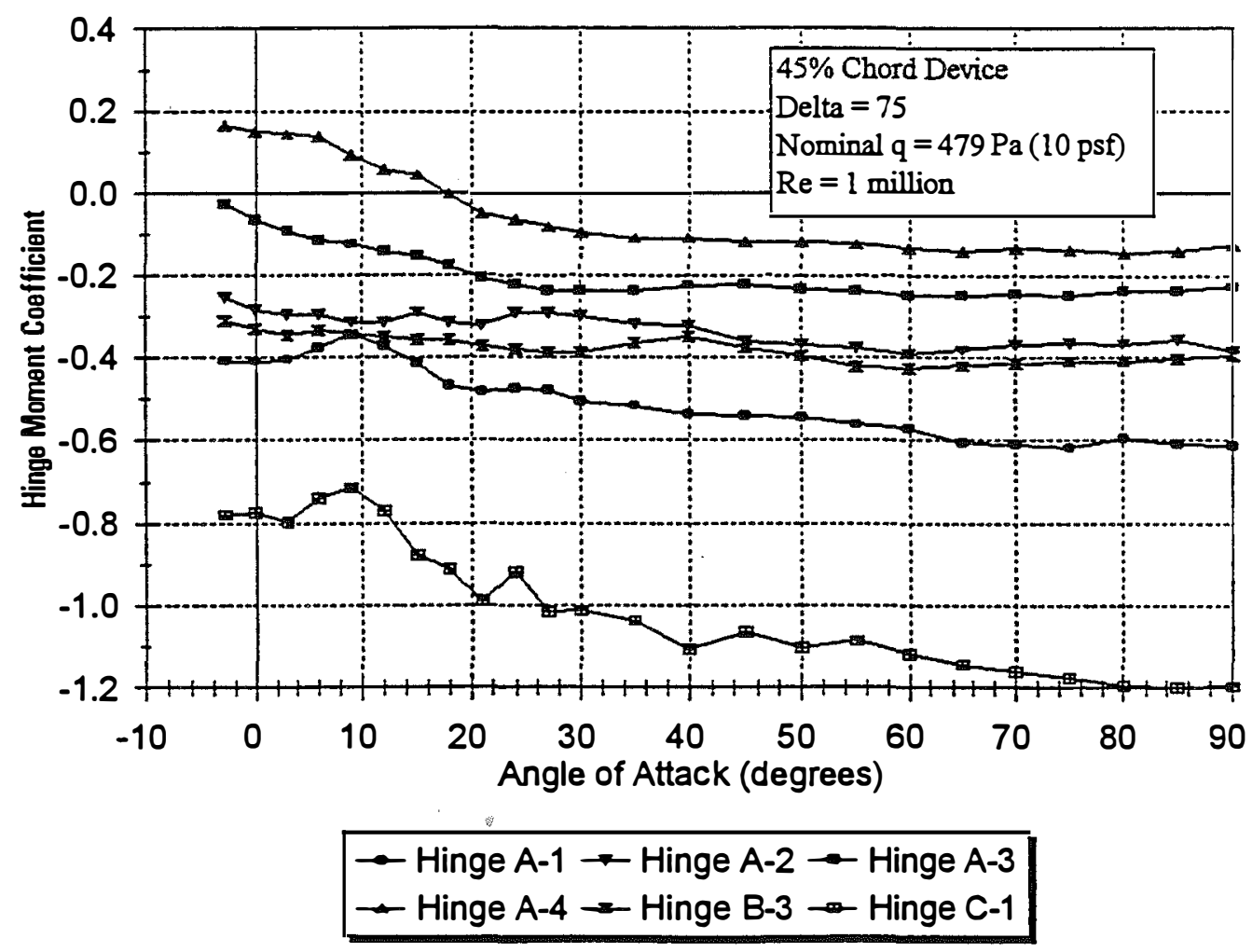

Figure A-23d. Hinge Moment Data, Effect of Hinge Location on Device at $\delta=75^{\circ}$ 


\section{APPENDLX B}

\section{Documentation of Cost and Weight}

Estimates 
Spoiler-Flap Active Mechanical System, Pneumatic/Bellcrank, Hinge A-3, Cost Analysis

\begin{tabular}{|c|c|c|c|c|c|c|c|c|c|c|}
\hline \multirow[t]{2}{*}{ Qty. } & \multirow{2}{*}{$\begin{array}{l}\text { Assemblyl } \\
\text { Part No. }\end{array}$} & \multirow{2}{*}{$\begin{array}{l}\text { Cost } \\
\text { Basls }\end{array}$} & \multirow[t]{2}{*}{ Descriptlon } & \multicolumn{6}{|c|}{$\cos (\$)$} & \multirow{2}{*}{$\begin{array}{l}\text { Notes } \\
\text { (l) = labor }\end{array}$} \\
\hline & & & & Parts & ext. & $\begin{array}{l}\text { Hours per } \\
\text { assembly }\end{array}$ & $\begin{array}{l}\text { Total } \\
\text { Hours }\end{array}$ & ext. & Subtotal & \\
\hline 1 & $x \times 10000$ & (l) & System Assy. & & - & 1.0 & 1.0 & 35.00 & 35.00 & $\begin{array}{l}\text { (I) connect pneumatic fittings, rig } \\
\text { spoiler-flap }\end{array}$ \\
\hline 2 & $x \times 63000$ & (l) & Blade Assy. & & - & 0.5 & 1.0 & 35.00 & 35.00 & $\begin{array}{l}\text { (I) install spoiler-flap, connect } \\
\text { pushrod }\end{array}$ \\
\hline 2 & $x \times 63000-1$ & 1 & Gas Spring, outboard & 16.00 & 32.00 & 0.1 & 0.2 & 7.00 & 39.00 & clip to ball studs \\
\hline 2 & $x \times 63000-1$ & 1 & Gas Spring, Inboard & 16.00 & 32.00 & 0.1 & 0.2 & 7.00 & 39.00 & clip to ball studs \\
\hline 2 & $x \times 63100$ & (I) & $43^{\prime}$ Blade, notched & & - & 8.00 & 16.0 & 560.00 & 560.00 & $\begin{array}{l}\text { (l) Cut out rough spoiler-flap, dress } \\
\text { edges, secure ribs and web } \\
\text { blocking, install bellcrank assy, } \\
\text { install cylinder attach bracket, } \\
\text { secure gap seals, install air hose } \\
\text { clips and air hose. }\end{array}$ \\
\hline 2 & $x \times 63100-1$ & 2 & Hose, Cylinder to Valve & 7.00 & 14.00 & 0.5 & 1.0 & 35.00 & 49.00 & $\begin{array}{l}40 \text { ' } x .10 / f t .+2 \text { fittings @ } \$ 3.00 \text { ea., } \\
\text { (I) assemble }\end{array}$ \\
\hline 2 & $x \times 63100-2$ & 1 & Pneumatic Cylinder & 114.70 & 229.40 & 0.4 & 0.8 & 28.00 & 257.40 & (I) secure with pins to brackets \\
\hline 2 & $x \times 63100-3$ & 5 & $\begin{array}{l}\text { Cylinder Attach Bracket, } \\
\text { with backup plate }\end{array}$ & 15.00 & 30.00 & 0.5 & 1.0 & 35.00 & 65.00 & $\begin{array}{l}\text { (I) drill web blocking, secure bracket } \\
\text { with } 4 \text { bolts }\end{array}$ \\
\hline 8 & $x \times 63100-4$ & 3 & Bracket Attach Bolts & 0.50 & 4.00 & & & - & 4.00 & \\
\hline 8 & $x \times 63100-5$ & 3 & Washers & 0.10 & 0.80 & & & - & 0.80 & \\
\hline 8 & $x \times 63100-6$ & 3 & Nuts & 0.25 & 2.00 & & & - & 2.00 & \\
\hline 2 & $x \times 63100-7$ & 3 & Clevis Pin & 1.00 & 2.00 & & & - & 2.00 & \\
\hline 30 & $x \times 63100-8$ & 1 & Gap seal & 0.55 & 16.50 & & & - & 16.50 & "p" seal, \$.55/ft. \\
\hline 2 & $x \times 63100-9$ & 2 & Thrust Bearing & 2.11 & 4.22 & & & & 4.22 & Torrington FNTA-2035 \\
\hline 8 & $x \times 63100-1$ & 2 & Thrust Washer & 0.64 & 5.12 & & & & 5.12 & INA AS2035 \\
\hline 4 & $x \times 63100-1$ & 3 & Hinge Bolt & 2.50 & 10.00 & & & & 10.00 & \\
\hline 4 & $x \times 63100-1$ & 3 & Castellated Nut & 0.95 & 3.80 & & & & 3.80 & \\
\hline 8 & $x \times 63100-1$ & 3 & Washer & 0.35 & 2.80 & & & & 2.80 & \\
\hline 30 & $x \times 63100-1$ & 2 & hose guide eyelets & 0.35 & 10.50 & & & & 10.50 & \\
\hline
\end{tabular}




\begin{tabular}{|c|c|c|c|c|c|c|c|c|c|c|}
\hline \multirow[t]{2}{*}{ Qty. } & \multirow{2}{*}{$\begin{array}{l}\text { Assemblyl } \\
\text { Part No. }\end{array}$} & \multirow{2}{*}{$\begin{array}{l}\text { Cost } \\
\text { Basis }\end{array}$} & \multirow[t]{2}{*}{ Descriptlon } & \multicolumn{6}{|c|}{$\cos T(\$)$} & \multirow{2}{*}{$\begin{array}{l}\text { Notes } \\
(0) \text { labor }\end{array}$} \\
\hline & & & & Parts & ext. & $\begin{array}{l}\text { Hours per } \\
\text { assembly }\end{array}$ & $\begin{array}{l}\text { Total } \\
\text { Hours } \\
\end{array}$ & ext. & Subtotal & \\
\hline 2 & $x \times 63110$ & 4 & $\begin{array}{l}\text { Blade Rib, inboard hinge } \\
\text { support, laminated wood }\end{array}$ & 9.12 & 18.24 & & & - & 18.24 & \\
\hline 2 & $x \times 63130$ & 4 & $\begin{array}{l}\text { Blade Hinge Rib, outboard } \\
\text { hinge support, laminated } \\
\text { wood }\end{array}$ & 3.50 & 7.00 & & & - & 7.00 & \\
\hline 2 & $x \times 63140$ & 5 & $\begin{array}{l}\text { Web blocking, cylinder } \\
\text { attach bracket }\end{array}$ & 2.00 & 4.00 & & & - & 4.00 & \\
\hline 2 & $x \times 63150$ & 5 & $\begin{array}{l}\text { Web blocking, bellcrank } \\
\text { bracket }\end{array}$ & 2.00 & 4.00 & & & - & 4.00 & \\
\hline 2 & $x \times 63200$ & (I) & Spoiler-Flap Assy. & & $\begin{array}{l}- \\
-\end{array}$ & 1.5 & 3.0 & 105.00 & 105.00 & $\begin{array}{l}\text { (l) dress edges, bond ribs and } \\
\text { webs, seal interior surfaces with } \\
\text { epoxy }\end{array}$ \\
\hline 2 & $x \times 63210$ & (l) & Hinge Rib Assy, Inboard & & - & 1.0 & 2.0 & 70.00 & 70.00 & $\begin{array}{l}\text { (l)install gas strut mount stud, bore } \\
\text { rib at hinge location, install thrust } \\
\text { washer and thrust stud }\end{array}$ \\
\hline 2 & $x \times 63210-1$ & 2 & $\begin{array}{l}\text { Gas Spring Connecting } \\
\text { Bracket }\end{array}$ & 0.70 & 1.40 & 0.2 & 0.4 & 14.00 & 15.40 & (l) secure to rib \\
\hline 2 & $x \times 63211$ & 4 & $\begin{array}{l}\text { Hinge Rib, Inboard, } \\
\text { laminated wood }\end{array}$ & 1.45 & 2.90 & & & - & 2.90 & \\
\hline 2 & $x \times 63220-1$ & 3 & Clevis Bolt, pushrod attach & 2.50 & 5.00 & & & - & 5.00 & \\
\hline 2 & $x \times 63220-2$ & 3 & Nut, Castellated & 0.50 & 1.00 & & & - & 1.00 & \\
\hline 4 & $x \times 63220-3$ & 3 & Washer & 0.10 & 0.40 & & & - & 0.40 & \\
\hline 2 & $x \times 63230$ & (l) & Hinge Rib Assy, Outboard & & & 1.0 & 2.0 & 70.00 & 70.00 & $\begin{array}{l}\text { (I) install gas strut mount stud, bore } \\
\text { rib at hinge location, install thrust } \\
\text { washer and thrust stud }\end{array}$ \\
\hline 8 & $x \times 63232$ & 3 & Hinge Stud & 0.84 & 6.72 & & & & 6.72 & \\
\hline 2 & $x \times 63230-1$ & 2 & $\begin{array}{l}\text { Gas Spring Connecting } \\
\text { Bracket }\end{array}$ & 0.99 & 1.98 & 0.2 & 0.4 & 14.00 & 15.98 & (I) secure to rib \\
\hline 2 & $x \times 63231$ & 4 & $\begin{array}{l}\text { Hinge Rib, outboard, } \\
\text { laminated wood }\end{array}$ & 0.84 & 1.68 & & & - & 1.68 & \\
\hline 2 & $x \times 63240$ & 4 & Web, Inboard & 5.00 & 10.00 & & & - & 10.00 & 1/4" birch ply \\
\hline
\end{tabular}




\begin{tabular}{|c|c|c|c|c|c|c|c|c|c|c|}
\hline \multirow[t]{2}{*}{ Qty. } & \multirow{2}{*}{$\begin{array}{l}\text { Assemblyl } \\
\text { Part No. }\end{array}$} & \multirow{2}{*}{$\begin{array}{l}\text { Cost } \\
\text { Basls }\end{array}$} & \multirow[t]{2}{*}{ Descriptlon } & \multicolumn{6}{|c|}{$\operatorname{cosT}(\$)$} & \multirow{2}{*}{$\begin{array}{l}\text { Notes } \\
(1)=\text { labor }\end{array}$} \\
\hline & & & & Parts & ext. & $\begin{array}{l}\text { Hours per } \\
\text { assembly }\end{array}$ & $\begin{array}{l}\text { Total } \\
\text { Hours }\end{array}$ & ext. & Subtotal & \\
\hline 2 & $x \times 63250$ & 4 & Web, Outboard & 5.00 & 10.00 & & & - & 10.00 & 1/4" birch ply \\
\hline 2 & $x \times 63300$ & (l) & Pushrod Assy. & & - & 0.2 & 0.4 & 14.00 & 14.00 & (I) assemble \\
\hline 2 & $x \times 63300-1$ & 2 & Clevis & 7.00 & 14.00 & & & - & 14.00 & \\
\hline 2 & $x \times 63300-2$ & 2 & Stop Nut & 0.50 & 1.00 & & & - & 1.00 & \\
\hline 2 & $x \times 63300-3$ & 2 & Rod End, Solid Stem & 10.50 & 21.00 & & & - & 21.00 & \\
\hline 2 & $x \times 63300-4$ & 2 & Rivet, rod end locking & 0.70 & 1.40 & & & - & 1.40 & \\
\hline 2 & $x \times 63400$ & 3 & Bellcrank Assy. & & - & 0.3 & 0.6 & 21.00 & 21.00 & (I) assemble \\
\hline 2 & $x \times 63400-1$ & 2 & Bearing, cylinder connect & 8.40 & 16.80 & & & - & 16.80 & \\
\hline 2 & $x \times 63400-2$ & 2 & Bearing, pushrod connect & 17.50 & 35.00 & & & - & 35.00 & \\
\hline 2 & $x \times 63400-3$ & 2 & Bearing, bellcrank pivot & 8.40 & 16.80 & & & - & 16.80 & \\
\hline 2 & $x \times 63400-4$ & 3 & Bolt, bellcrank pivot & 2.50 & 5.00 & & & - & 5.00 & \\
\hline 2 & $x \times 63400-5$ & 3 & Nut, bellcrank pivot & 0.50 & 1.00 & & & - & 1.00 & \\
\hline 4 & $x \times 63400-6$ & 3 & Washer, bellcrank pivot & 0.10 & 0.40 & & & - & 0.40 & \\
\hline 8 & $x \times 63400-7$ & 3 & Bolt, bellcrank mount & 0.50 & 4.00 & & & - & 4.00 & \\
\hline 8 & $x \times 63400-8$ & 3 & Nut, bellcrank mount & 0.25 & 2.00 & & & - & 2.00 & \\
\hline 16 & $x \times 63400-9$ & 3 & Washer, bellcrank mount & 0.10 & 1.60 & & & - & 1.60 & \\
\hline 2 & $x \times 63410$ & 5 & Bellcrank Machining & 36.00 & 72.00 & & & - & 72.00 & \\
\hline 2 & $x \times 63420$ & 5 & $\begin{array}{l}\text { Bellcrank Bracket, with } \\
\text { backup plate }\end{array}$ & 90.00 & 180.00 & & & - & 180.00 & \\
\hline 1 & $x \times 13000$ & (I) & Hub Assy. & & - & 2.0 & 2.0 & 70.00 & 70.00 & $\begin{array}{l}\text { (I) install compressor, tank, and } \\
\text { solenoid valve, install fuse box, wire } \\
\text { components }\end{array}$ \\
\hline 1 & $x \times 13000-2$ & 1 & Compressor & 329.70 & 329.70 & & & - & 329.70 & \\
\hline 1 & $x \times 13000-3$ & 2 & $\begin{array}{l}\text { Custom air tank with mount } \\
\text { brackets }\end{array}$ & 105.00 & 105.00 & & & - & 105.00 & \\
\hline 1 & $x \times 13000-4$ & 1 & Solenoid Valve & 18.80 & 18.80 & & & - & 18.80 & \\
\hline 1 & $x \times 13000-5$ & 2 & Hose, Compressor to Tank & 7.00 & 7.00 & 0.5 & 0.5 & 17.50 & 24.50 & \\
\hline 1 & $x \times 13000-6$ & 2 & Hose, Tank to Valve & 7.00 & 7.00 & 0.5 & 0.5 & 17.50 & 24.50 & \\
\hline
\end{tabular}




\begin{tabular}{|c|c|c|c|c|c|c|c|c|c|c|}
\hline \multirow[t]{2}{*}{ Qty. } & \multirow{2}{*}{$\begin{array}{l}\text { Assemblyl } \\
\text { Part No. }\end{array}$} & \multirow{2}{*}{$\begin{array}{l}\text { Cost } \\
\text { Basls }\end{array}$} & \multirow[t]{2}{*}{ Description } & \multicolumn{6}{|c|}{$\operatorname{cost}(\$)$} & \multirow{2}{*}{$\begin{array}{l}\text { Notes } \\
(I)=\text { labor }\end{array}$} \\
\hline & & & & Parts & ext. & $\begin{array}{l}\text { Hours per } \\
\text { assembly }\end{array}$ & $\begin{array}{l}\text { Total } \\
\text { Hours }\end{array}$ & ext. & Subtotal & \\
\hline 1 & $x \times 13100$ & (l) & Hub, Machined & & - & 0.8 & 0.8 & 28.00 & 28.00 & $\begin{array}{l}\text { (I) additional drilling for pneumatic } \\
\text { system components }\end{array}$ \\
\hline NA & & 6 & $\begin{array}{l}\text { Unspecified parts and } \\
\text { processes }\end{array}$ & 200.00 & 200.00 & & 5.0 & 175.00 & 375.00 & \\
\hline
\end{tabular}

System Totals $\quad \$ 1,517 \quad 39.6 \$ 1,386 \$ 2,903$ Installed cost, 2 spoller-flaps

Legend for cost basis:

Based on price quote for part quantity required for 100 AWT wind turbines

Catalog price less assumed $30 \%$ discount for quantity purchase

Cost estimate based on AWT production invoices for comparable items

Cost estimated based on dollars per pound of production AWT wood-epoxy blades

Cost estimated based on dollars per pound of production AWT tip-vane hinge assembly

6 Cost estimate based on design and manufacturing experience of engineers at AWT and Advanced Blade

Manufacturing, Inc. Due to the preliminary nature of the design, additional labor and material costs

(unspecified parts and processes) are anticipated. Design details which may increase costs include:

- Addition of access panels for Inspection and maintenance of actuator system

- High-durability weather proofing of all cut wood-epoxy surfaces (blade and flap)

- Addition of damping or cushions to prevent hard mechanical stop of spoiler-flap during deployment

- Additional cost of properly sealing spanwise gaps and end gaps to ensure smooth aerodynamic surfaces and minimal noise during turbine operation

- Possible need for close-tolerance bolts

- Possible need for structural reinforcement of blade or spoiler-flap

- Addition of position sensor/switch for input to controller 


\section{Spoiler-Flap Active Mechanical System, Pneumatic/Bellcrank, Hinge A-3 Weight Analysis}

\begin{tabular}{|c|c|c|c|c|c|c|c|}
\hline Qty. & $\begin{array}{l}\text { Assemblyl } \\
\text { Part No. }\end{array}$ & Description & Wt. (lb) & ext. & subtot. & Total & Notes \\
\hline 1 & $x \times 10000$ & System Assy. & & & & & \\
\hline 2 & $x \times 63000$ & Blade Assy. & & & & & \\
\hline 2 & $x \times 63000-1$ & Gas Spring, outboard & 2.00 & 4.00 & & & \\
\hline 2 & $x \times 63000-1$ & Gas Spring, inboard & 2.00 & 4.00 & & & \\
\hline 4 & $x \times 63000-2$ & Gas Spring Connecting Bracket & 0.10 & 0.40 & & & \\
\hline 2 & $x \times 63100$ & 43' Blade, notched & & & & & \\
\hline 2 & $x \times 63100-1$ & Hose, Cylinder to Valve & 1.20 & 2.40 & & & \\
\hline 2 & $x \times 63100-2$ & Pneumatic Cylinder & 11.10 & 22.20 & & & \\
\hline 2 & $x \times 63100-3$ & $\begin{array}{l}\text { Cylinder Attach Bracket, with backup } \\
\text { plate }\end{array}$ & 0.50 & 1.00 & & & \\
\hline 8 & $x \times 63100-4$ & Bracket Attach Bolts & 0.02 & 0.16 & & & \\
\hline 8 & $x \times 63100-5$ & Washers & 0.01 & 0.08 & & & \\
\hline 8 & $x \times 63100-6$ & Nuts & 0.01 & 0.08 & & & \\
\hline 2 & $x \times 63100-7$ & Clevis Pin & 0.10 & 0.20 & & & \\
\hline 30 & $x \times 63100-8$ & Gap seal & 0.10 & 3.00 & & & \\
\hline 2 & $x \times 63100-9$ & Thrust Bearing & 0.10 & 0.20 & & & \\
\hline 8 & $x \times 63100-10$ & Thrust Washer & 0.05 & 0.40 & & & \\
\hline 4 & $x \times 63100-11$ & Hinge Bolt & 0.10 & 0.40 & & & \\
\hline 4 & $x \times 63100-12$ & Castellated Nut & 0.05 & 0.20 & & & \\
\hline 8 & $x \times 63100-13$ & Washer & 0.02 & 0.16 & & & \\
\hline 30 & $x \times 63100-14$ & hose guide eyelets & 0.01 & 0.30 & & & \\
\hline 2 & $x \times 63110$ & $\begin{array}{l}\text { Blade Rib, inboard hinge support, } \\
\text { laminated wood } 1 / 2 \text { in thick }\end{array}$ & 3.50 & 7.00 & & & \\
\hline 2 & $x \times 63130$ & $\begin{array}{l}\text { Blade Hinge Rib, outboard hinge } \\
\text { support, laminated wood } 1 / 2 \text { in thick }\end{array}$ & 2.00 & 4.00 & & & \\
\hline
\end{tabular}




\begin{tabular}{|c|c|c|c|c|c|c|c|}
\hline Qty. & $\begin{array}{l}\text { Assemblyl } \\
\text { Part No. }\end{array}$ & Description & Wt. (lb) & ext. & subtot. & Total & Notes \\
\hline 2 & $x \times 63140$ & $\begin{array}{l}\text { Web blocking, cylinder attach } \\
\text { bracket }\end{array}$ & 0.50 & 1.00 & & & \\
\hline 2 & $x \times 63150$ & Web blocking, bellcrank bracket & 0.50 & 1.00 & & & \\
\hline 2 & $x \times 63200$ & Spoiler-Flap Assy. & & & & & \\
\hline 2 & $x \times 63210-1$ & Gas Spring Connecting Bracket & 0.10 & 0.20 & & & \\
\hline 2 & $x \times 63211$ & Hinge Rib, inboard, laminated wood & 2.00 & 4.00 & & & \\
\hline 2 & $x \times 63220-1$ & Clevis Bolt, pushrod attach & 0.10 & 0.20 & & & \\
\hline 2 & $x \times 63220-2$ & Nut, Castellated & 0.05 & 0.10 & & & \\
\hline 4 & $x \times 63220-3$ & Washer & 0.02 & 0.08 & & & \\
\hline 8 & $x \times 63232$ & Hinge Stud & 0.74 & 5.92 & & & \\
\hline 2 & $x \times 63230-1$ & Gas Spring Connecting Bracket & 0.10 & 0.20 & & & \\
\hline 2 & $x \times 63231$ & Hinge Rib, outboard, laminated wood & 1.00 & 2.00 & & & \\
\hline 2 & $x \times 63240$ & Web, Inboard & 1.00 & 2.00 & & & \\
\hline 2 & $x \times 63250$ & Web, Outboard & 1.00 & 2.00 & & & \\
\hline 2 & $x \times 63300$ & Pushrod Assy. & 1.50 & 3.00 & & & \\
\hline 2 & $x \times 63300-1$ & Clevis & 0.10 & 0.20 & & & \\
\hline 2 & $x \times 63300-2$ & Stop Nut & 0.02 & 0.04 & & & \\
\hline 2 & $x \times 63300-3$ & Rod End, Solid Stem & 0.10 & 0.20 & & & \\
\hline 2 & $x \times 63300-4$ & Rivet, rod end locking & 0.01 & 0.02 & & & \\
\hline 2 & $x \times 63400$ & Bellcrank Assy. & & & & & \\
\hline 2 & $x \times 63400-1$ & Bearing, cylinder connect & 0.01 & 0.02 & & & \\
\hline 2 & $x \times 63400-2$ & Bearing, pushrod connect & 0.01 & 0.02 & & & \\
\hline 2 & $x \times 63400-3$ & Bearing, bellcrank pivot & 0.06 & 0.12 & & & \\
\hline 2 & $x \times 63400-4$ & Bolt, bellcrank pivot & 0.05 & 0.10 & & & \\
\hline 2 & $x \times 63400-5$ & Nut, belicrank pivot & 0.02 & 0.04 & & & \\
\hline 4 & $x \times 63400-6$ & Washer, bellcrank pivot & 0.01 & 0.04 & & & \\
\hline
\end{tabular}




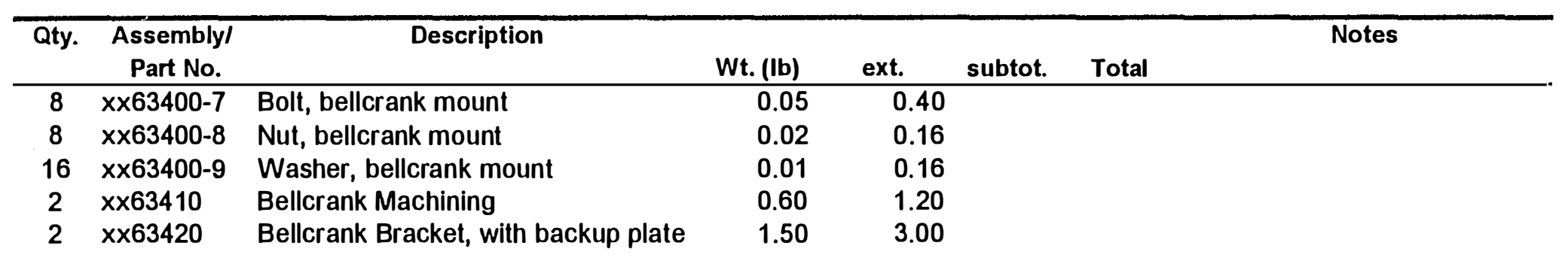

\section{Total Blade Assembly (2 blades)}

$\infty$
$\infty$
$\infty$

\begin{tabular}{|c|c|}
\hline$x \times 13000$ & Hub Assy. \\
\hline$x \times 13000-1$ & Hub Fuse Box \\
\hline$x \times 13000-2$ & Compressor \\
\hline$x \times 13000-3$ & Custom air tank with mount brackets \\
\hline$x \times 13000-4$ & Solenoid Valve \\
\hline$x \times 13000-5$ & Hose, Compressor to Tank \\
\hline$x \times 13000-6$ & Hose, Tank to Valve \\
\hline
\end{tabular}

Total Hub Assembly

Total Spoiler-Flap System
77.60

Total blade assembly weight is in addition to the baseline weight of AWT 26/27 woodepoxy blade.

$\begin{array}{rr}5.00 & 5.00 \\ 15.00 & 15.00 \\ 15.00 & 15.00 \\ & \\ 1.00 & 1.00 \\ 0.50 & 0.50 \\ 0.50 & 0.50\end{array}$

37.00

Total hub assembly weight is

in addition to the baseline

weight of AWT 26/27 hub.
114.60 


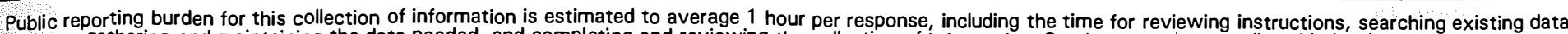

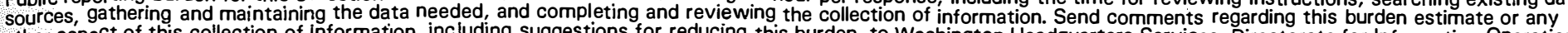

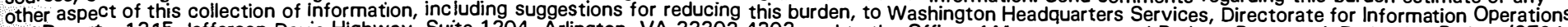

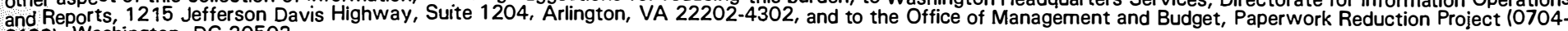
0188), Washington, DC 20503.

1.

April 1997
3. REPORT TYPE AND DATES COVERED

Subcontractor Report
4. TITLE AND SUBTITLE

Investigation of Aerodynamic Braking Devices for Wind Turbine Applications
5. FUNDING NUMBERS

C: ZAA-5-12272-05

TA: WE7 13010

6. AUTHOR(S)

D. A. Griffin

7. PERFORMING ORGANIZATION NAME(S) AND ADDRESS(ES)

R. Lynette \& Associates

425 Pontius Ave. North, Suite 150

Seattle, WA 98109
8. PERFORMING ORGANIZATION REPORT NUMBER

\section{SPONSORING/MONITORING AGENCY NAME(S) AND ADDRESS(ES)}

National Renewable Energy Laboratory

1617 Cole Blvd.

Golden, CO 80401-3393
10. SPONSORING/MONITORING AGENCY REPORT NUMBER

SR-440-22253

DE97000234

\section{SUPPLEMENTARY NOTES}

12a. DISTRIBUTION/AVAILABILITY STATEMENT

National Technical Information Service

U.S. Department of Commerce

5285 Port Royal Road

Springfield, VA 22161 12b. DISTRIBUTION CODE

UC-1213

\section{ABSTRACT (Maximum 200 words)}

This report documents the selection and preliminary design of a new aerodynamic braking system for use on the stall-regulated AWT-26/27 wind turbines. The goal was to identify and design a configuration that offered improvements over the existing tip brake used by Advanced Wind Turbines, Inc. Although the design objectives and approach of this report are specific to aerodynamic braking of AWT-26/27 turbines, many of the issues addressed in this work are applicable to a wider class of turbines. The performance trends and design choices presented in this report should be of general use to wind turbine designers who are considering alternative braking methods.

\section{SUBJECT TERMS}

15. NUMBER OF PAGES

renewable energy; wind energy; wind turbine design; wind turbines-electricity generation; wind turbine rotor design; trailing-edge devices; aerodynamic controls; spoiler-flap

16. PRICE CODE

17. SECURITY CLASSIFICATION OF REPORT

Unclassified
18. SECURITY CLASSIFICATION OF THIS PAGE Unclassified
19. SECURITY CLASSIFICATION OF ABSTRACT

Unclassified
20. LIMITATION OF ABSTRACT UL 